\title{
Synthesis and Biological Evaluation of Celastrol Derivatives with Improved Cytotoxic Selectivity and Antitumor Activities
}

Xiao-Long $\mathrm{Hu}^{\perp, \#}$, Qi-Wei He ${ }^{\perp, \#}$, Huan Long ${ }^{\perp}$, Li-Xin Zhang ${ }^{\perp}$, Rong Wang ${ }^{\perp}$, Bao-Lin Wang $^{\perp}$, Jia-Hao Feng ${ }^{\perp}$, Quan Wang ${ }^{\perp}$, Ji-Qin Hou ${ }^{\perp}$, Xiao-Qi Zhang ${ }^{\dagger}$, Wen-Cai Ye ${ }^{\dagger}$, and Hao Wang ${ }^{* \perp}$

${ }^{\perp}$ State Key Laboratory of Natural Medicines, School of Traditional Chinese Pharmacy, China Pharmaceutical University, Nanjing 210009, People’s Republic of China.

${ }^{\dagger}$ Institute of Traditional Chinese Medicine and Natural Products, Jinan University, Guangzhou 510632, People's Republic of China.

${ }^{*}$ Corresponding author: Hao Wang

E-mail: wanghao@cpu.edu.cn

Postal address: State Key Laboratory of Natural Medicines, China Pharmaceutical University, Nanjing 210009, People's Republic of China. 


\section{Table of contents}

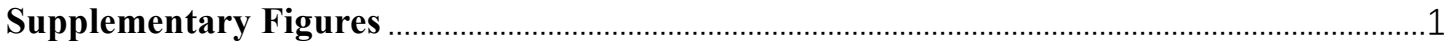

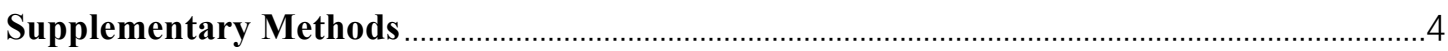

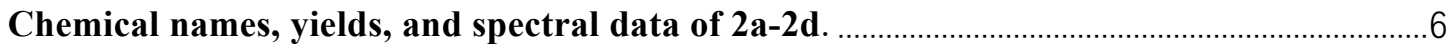

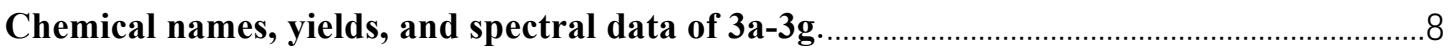

Chemical names, yields, and spectral data of 4a-4t. ........................................................ 12

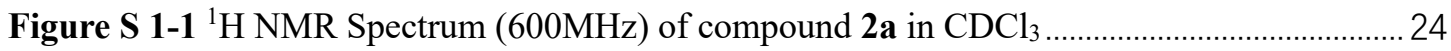

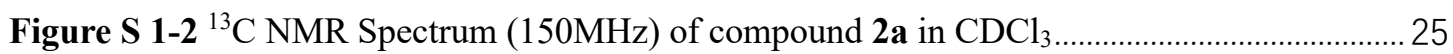

Figure S 1-4 HRESIMS Spectrum of compound 2a

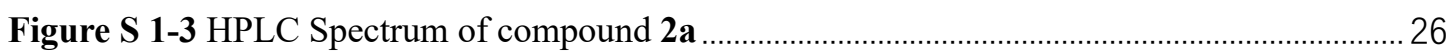

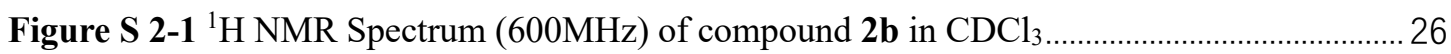

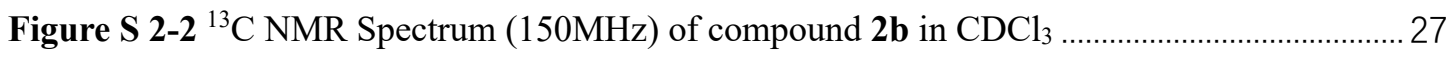

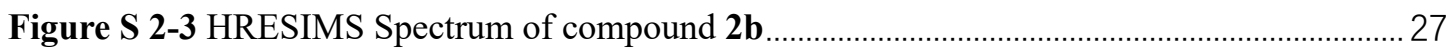

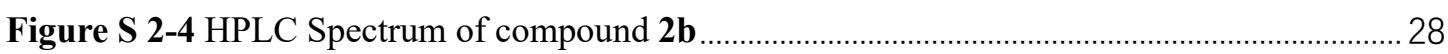

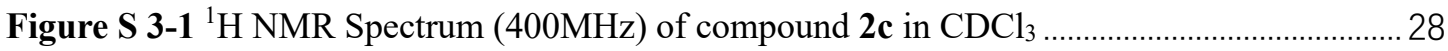

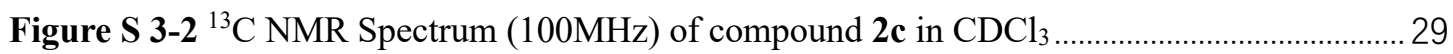

Figure S 3-3 HRESIMS Spectrum of 2c

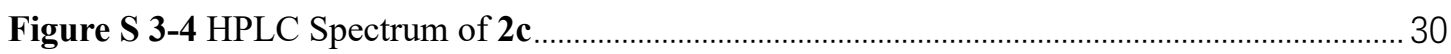

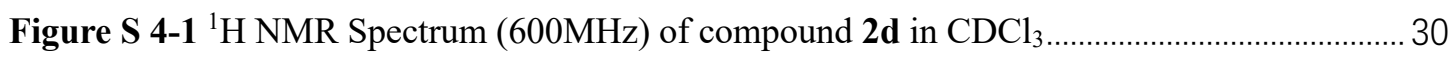

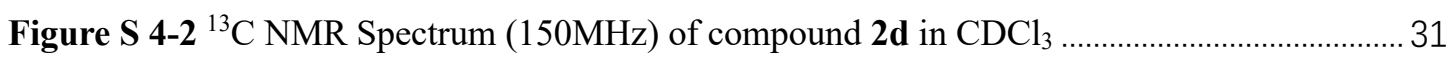

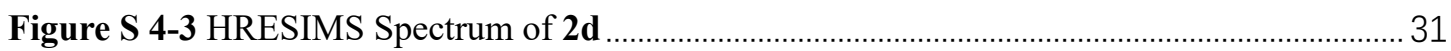

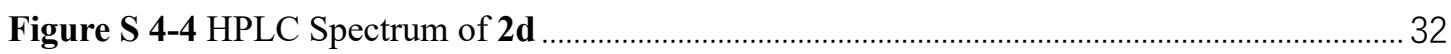

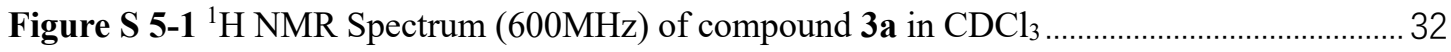

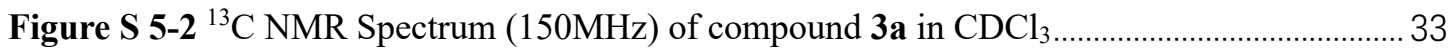

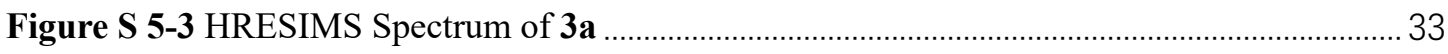

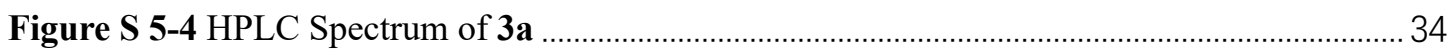

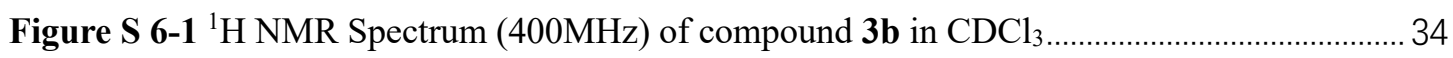

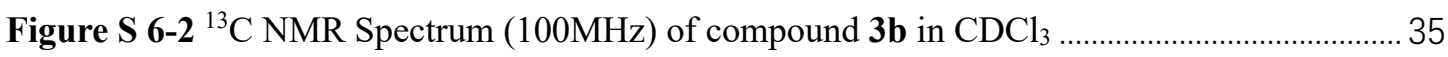

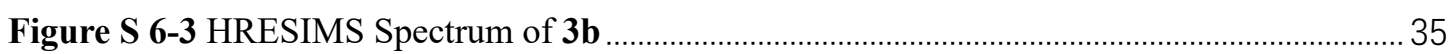

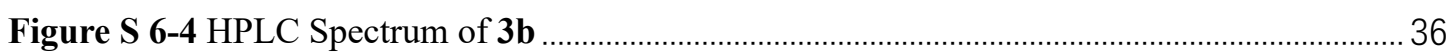

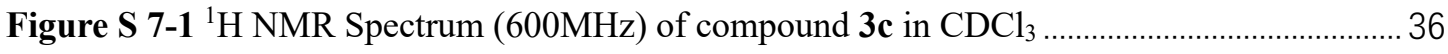

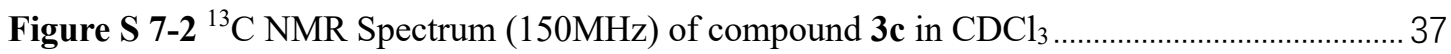

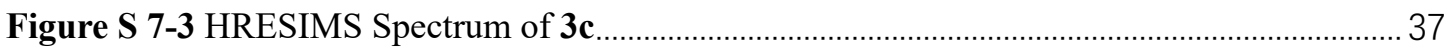

Figure S 7-4 HPLC Spectrum of 3c

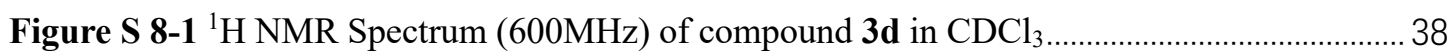

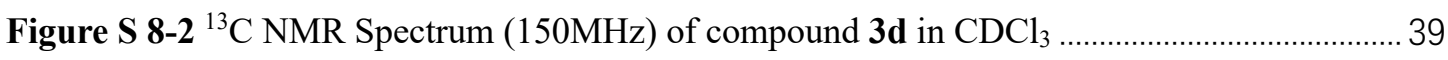

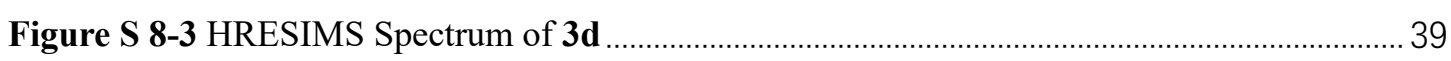

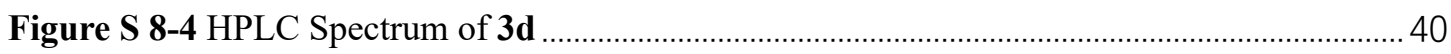

Figure S 9-1 ${ }^{1} \mathrm{H}$ NMR Spectrum $(600 \mathrm{MHz})$ of compound $\mathbf{3 e}$ in $\mathrm{CDCl}_{3} \ldots \ldots \ldots \ldots \ldots \ldots \ldots \ldots \ldots \ldots \ldots \ldots . . . . . . . . . . . . . . . . . .40$

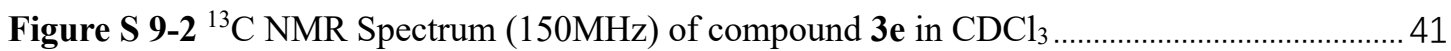

Figure S 9-3 HRESIMS Spectrum of 3e

Figure S 9-4 HPLC Spectrum of 3e

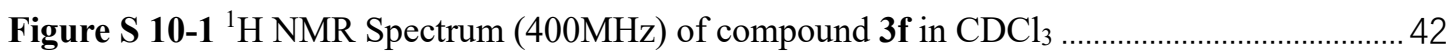


Figure S 10-2 ${ }^{13} \mathrm{C}$ NMR Spectrum $(100 \mathrm{MHz})$ of compound $\mathbf{3 f}$ in $\mathrm{CDCl}_{3} \ldots \ldots \ldots \ldots \ldots \ldots \ldots \ldots \ldots \ldots \ldots \ldots \ldots . . .43$

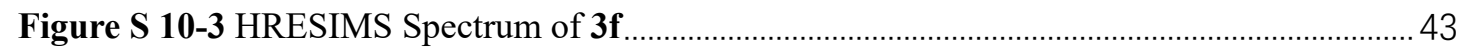

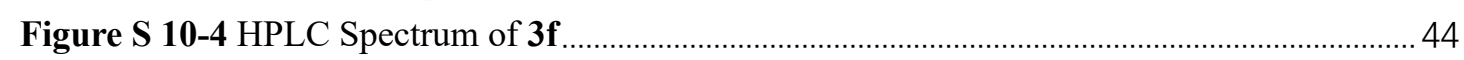

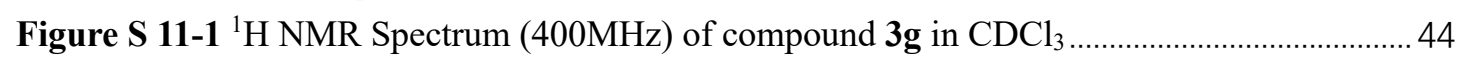

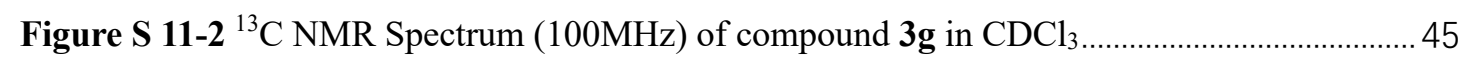

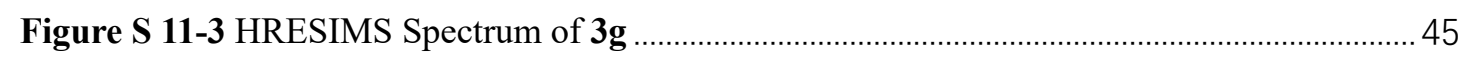

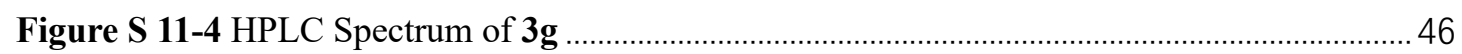

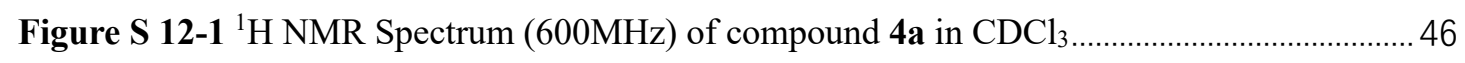

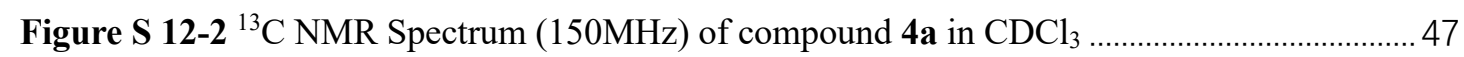

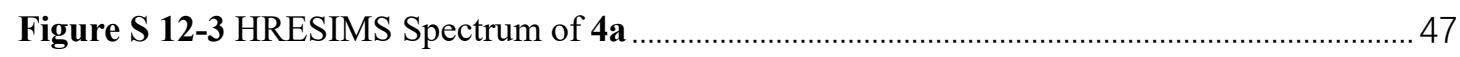

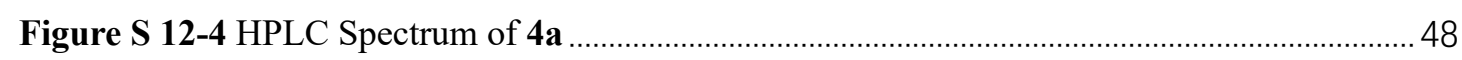

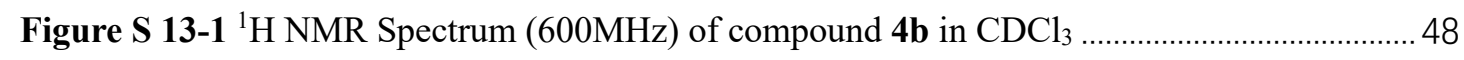

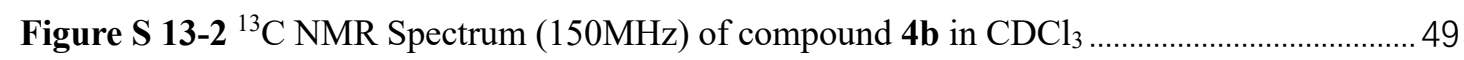

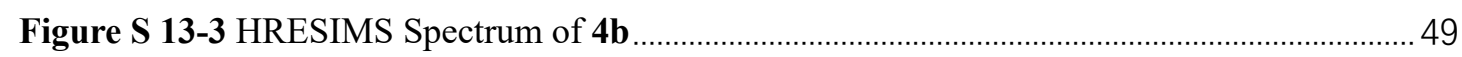

Figure S 13-4 HPLC Spectrum of $\mathbf{4 b}$........................................................................................... 50

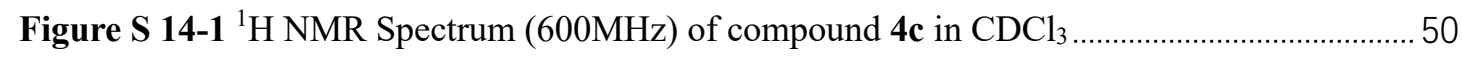

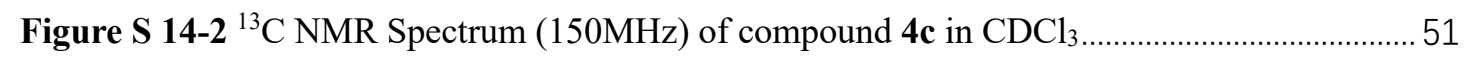

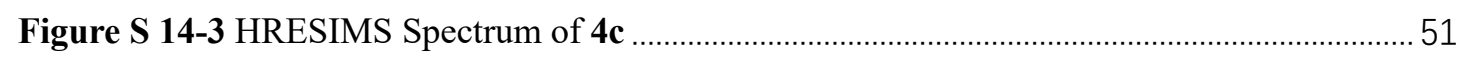

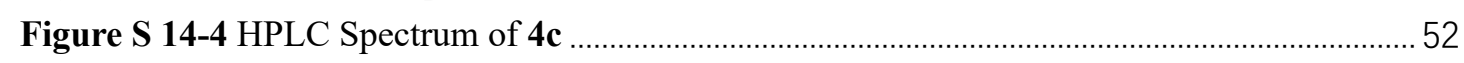

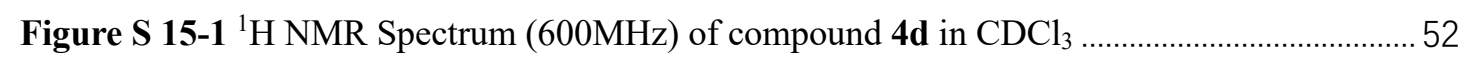

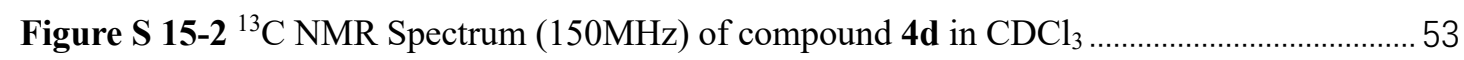

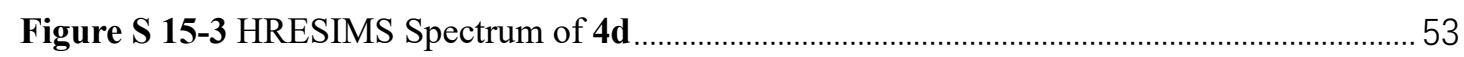

Figure S 15-4 HPLC Spectrum of $\mathbf{4 d}$........................................................................................ 54

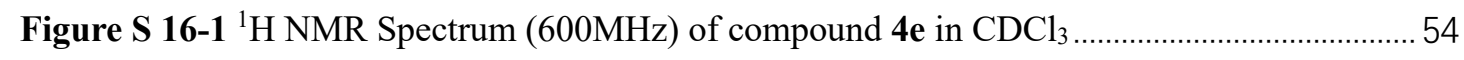

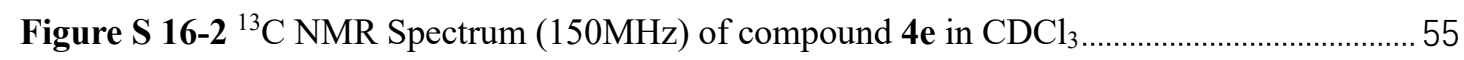

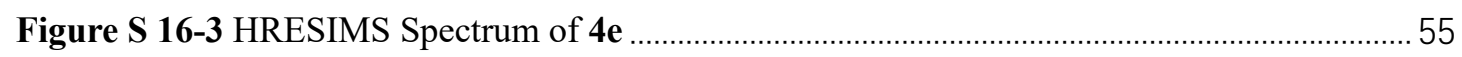

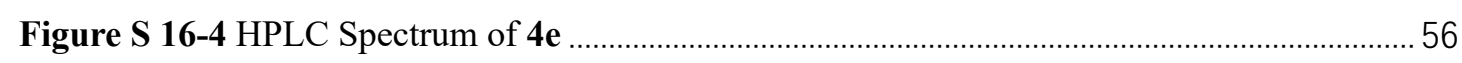

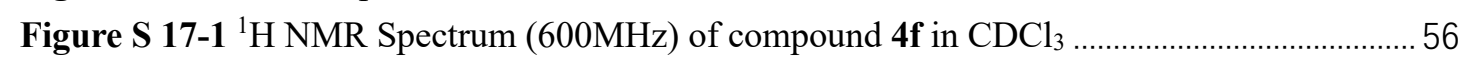

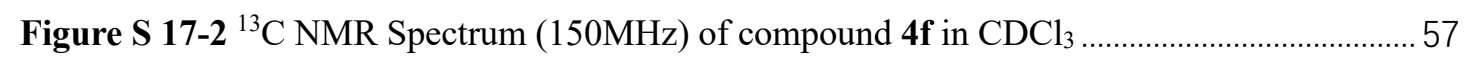

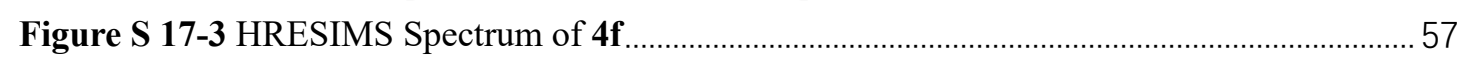

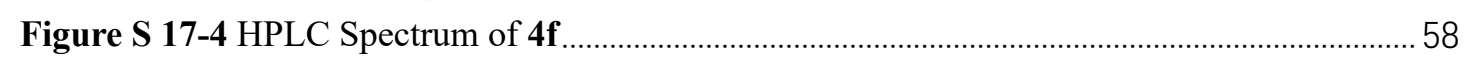

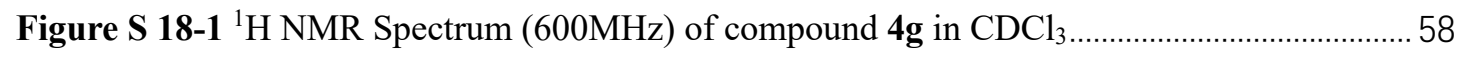

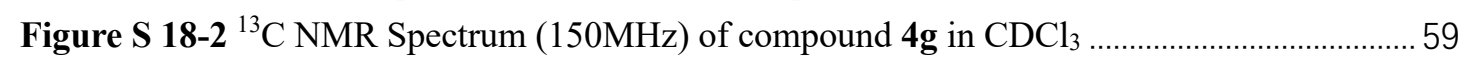

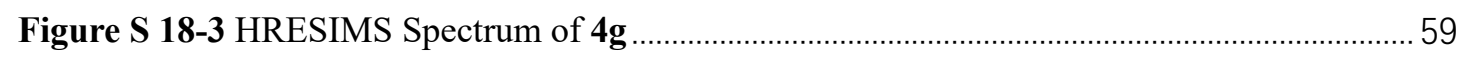

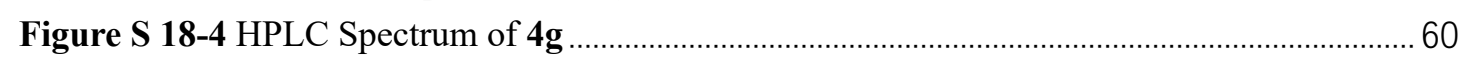

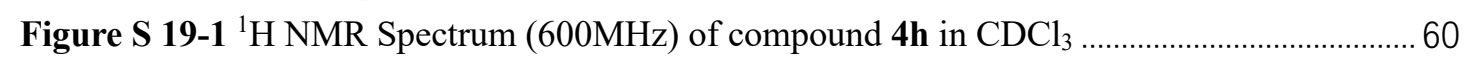

Figure S 19-2 ${ }^{13} \mathrm{C}$ NMR Spectrum $(150 \mathrm{MHz})$ of compound $\mathbf{4 h}$ in $\mathrm{CDCl}_{3} \ldots \ldots \ldots \ldots \ldots \ldots \ldots \ldots \ldots \ldots \ldots \ldots . . . . . . . . . . . . . . . .61$

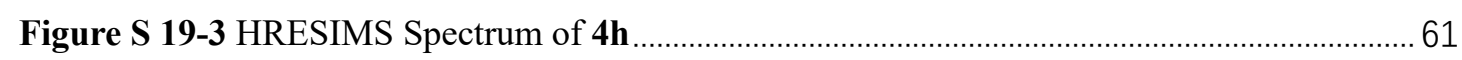

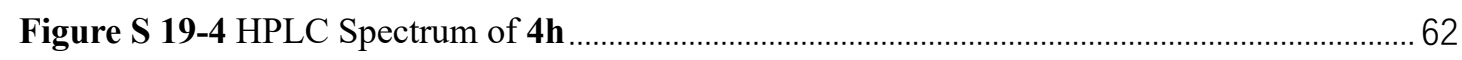

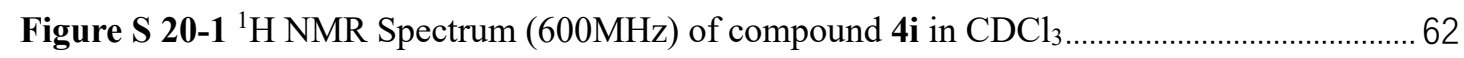

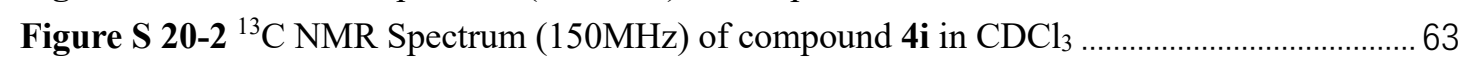

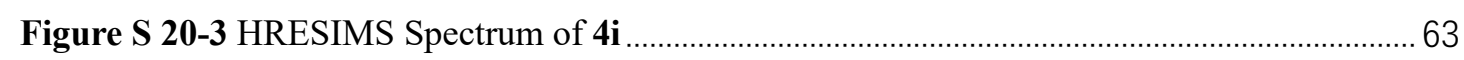

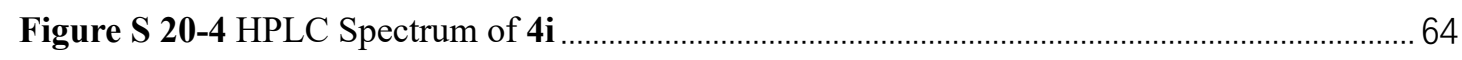

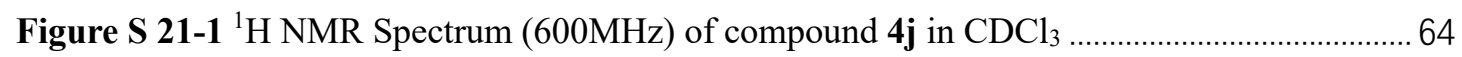




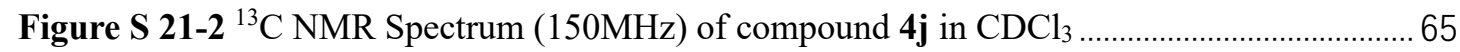

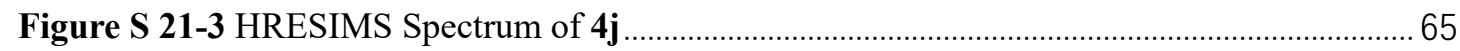

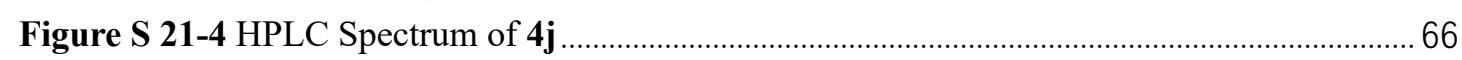

Figure S 22-1 ${ }^{1} \mathrm{H}$ NMR Spectrum (600MHz) of compound $\mathbf{4 k}$ in $\mathrm{CDCl}_{3} \ldots \ldots \ldots \ldots \ldots \ldots \ldots \ldots \ldots \ldots \ldots \ldots . . . . . . . . . . . . . . . . . . .66$

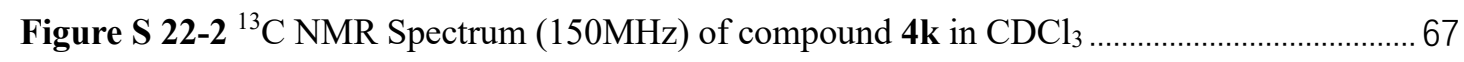

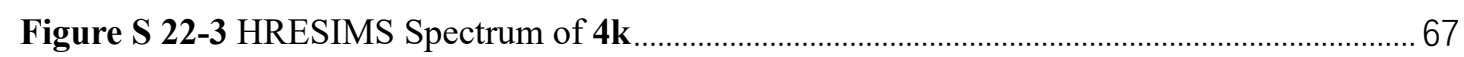

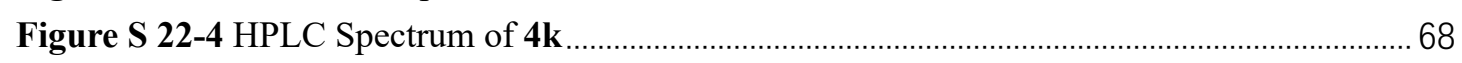

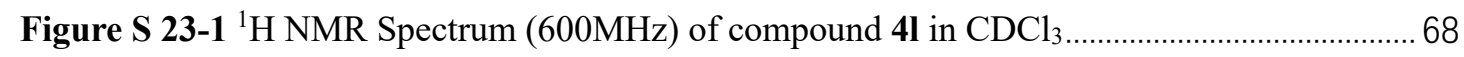

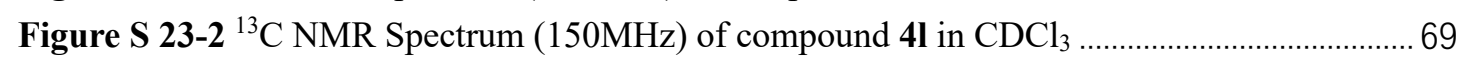

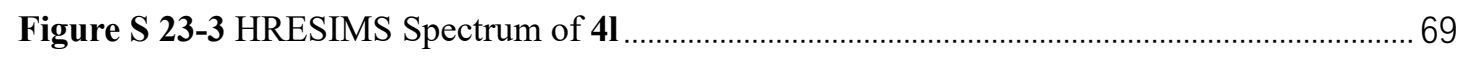

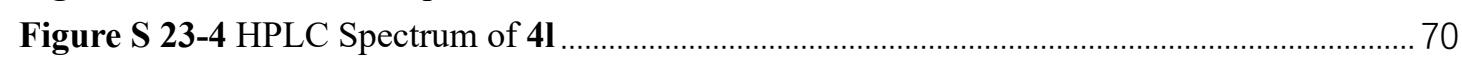

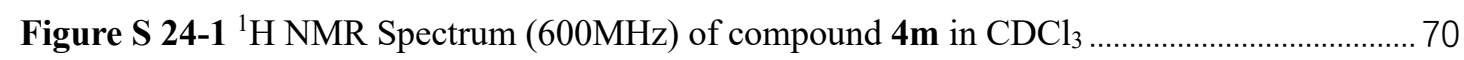

Figure S 24-2 ${ }^{13} \mathrm{C}$ NMR Spectrum $(150 \mathrm{MHz})$ of compound $\mathbf{4 m}$ in $\mathrm{CDCl}_{3} \ldots \ldots \ldots \ldots \ldots \ldots \ldots \ldots \ldots \ldots \ldots . . . . . . . . . . . . . . . . .71$

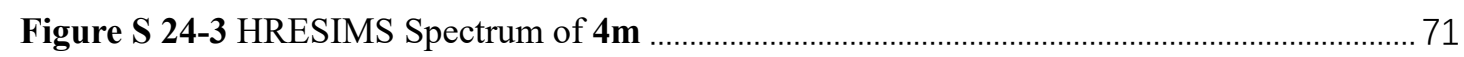

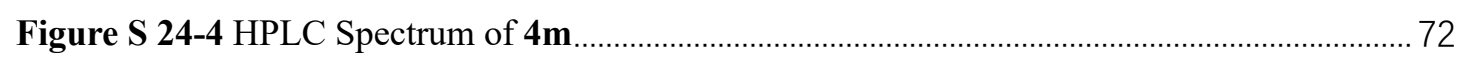

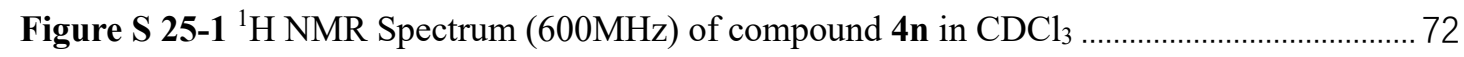

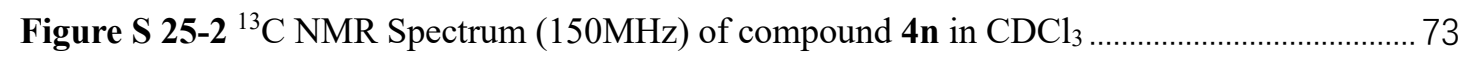

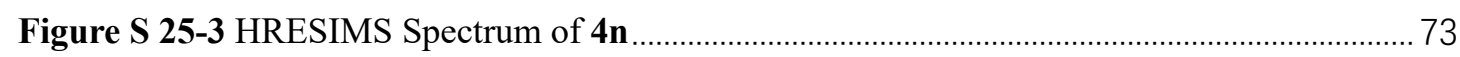

Figure S 25-4 HPLC Spectrum of 4n ……………….................................................................. 74

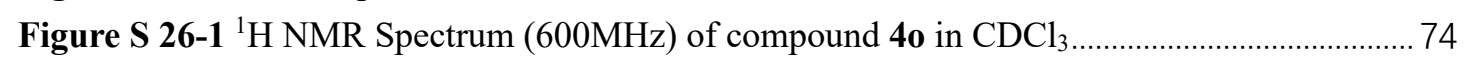

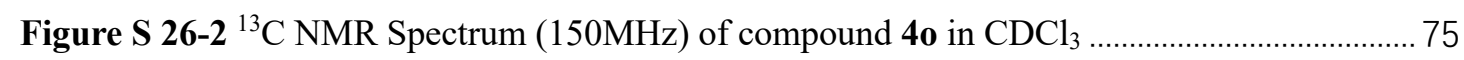

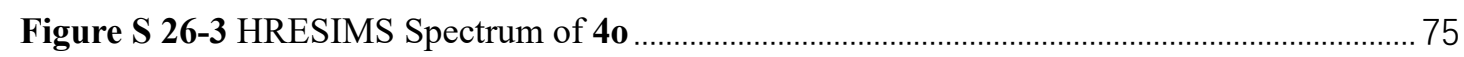

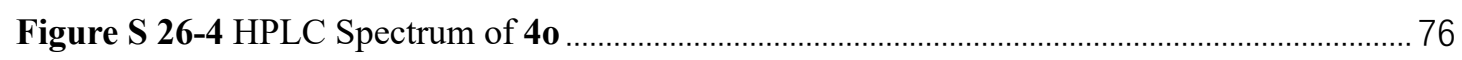

Figure S 27-1 ${ }^{1} \mathrm{H}$ NMR Spectrum (600MHz) of compound $\mathbf{4 p}$ in $\mathrm{CDCl}_{3} \ldots \ldots \ldots \ldots \ldots \ldots \ldots \ldots \ldots \ldots \ldots \ldots \ldots . . .76$

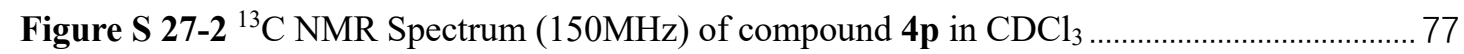

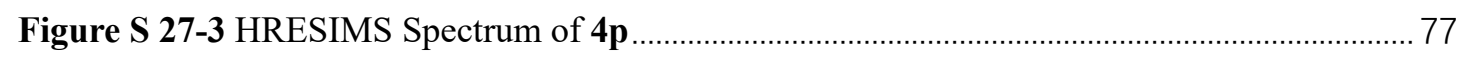

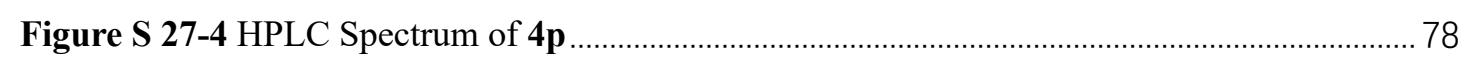

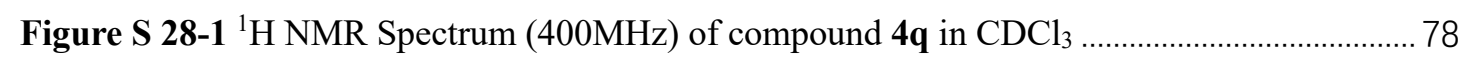

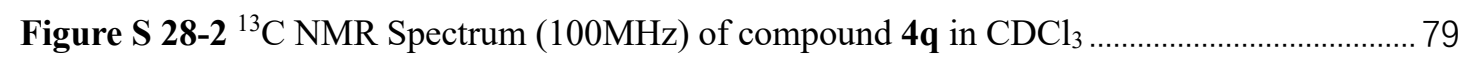

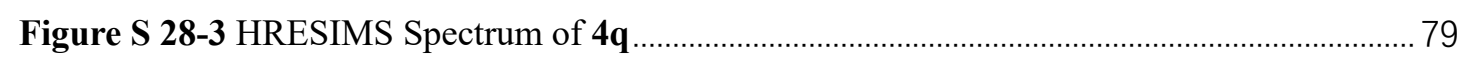

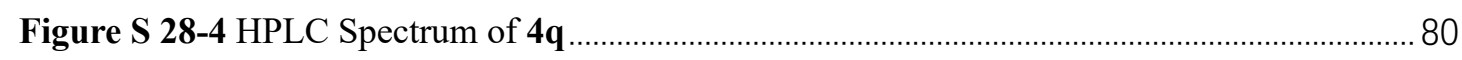

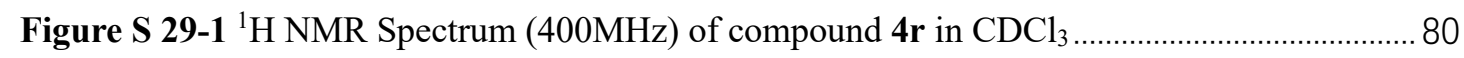

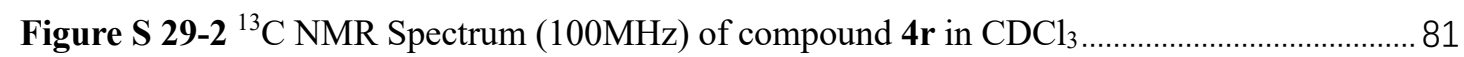

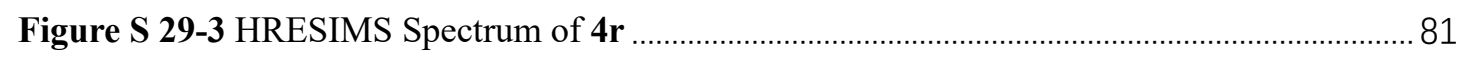

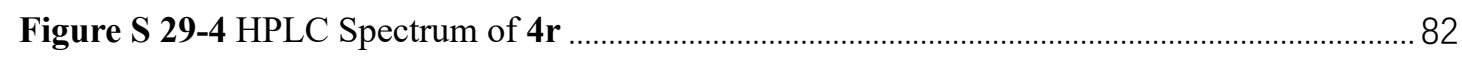

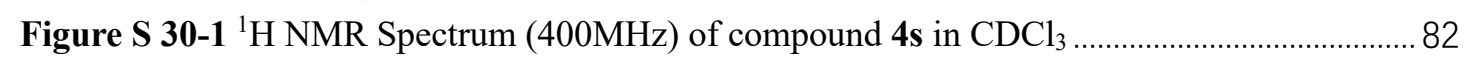

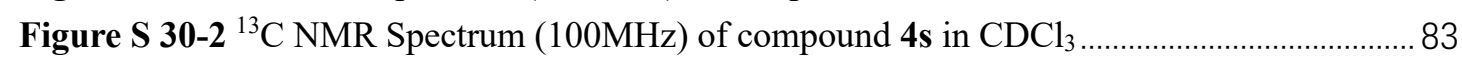

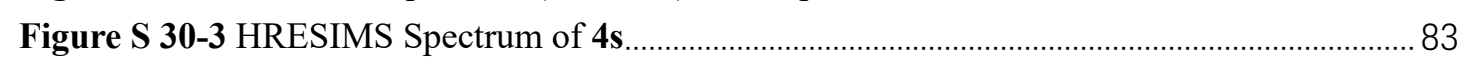

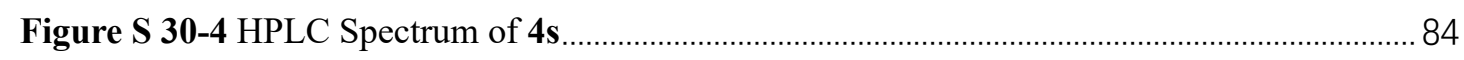

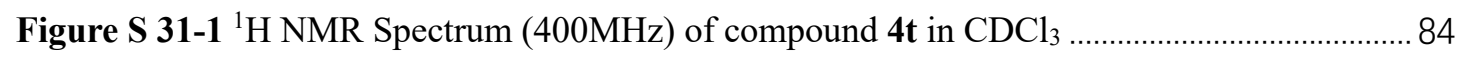

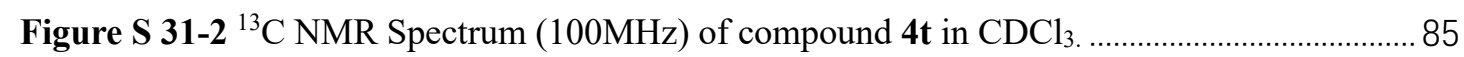

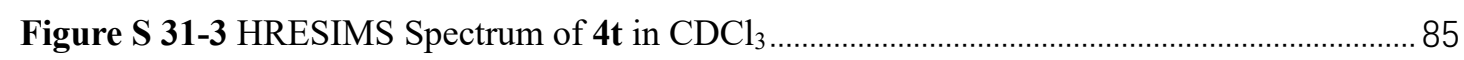

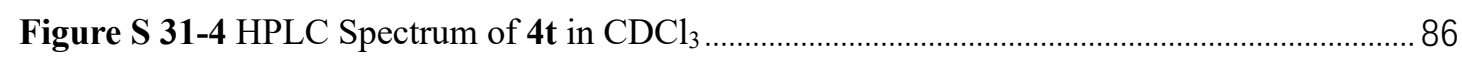




\section{Supplementary Figures}
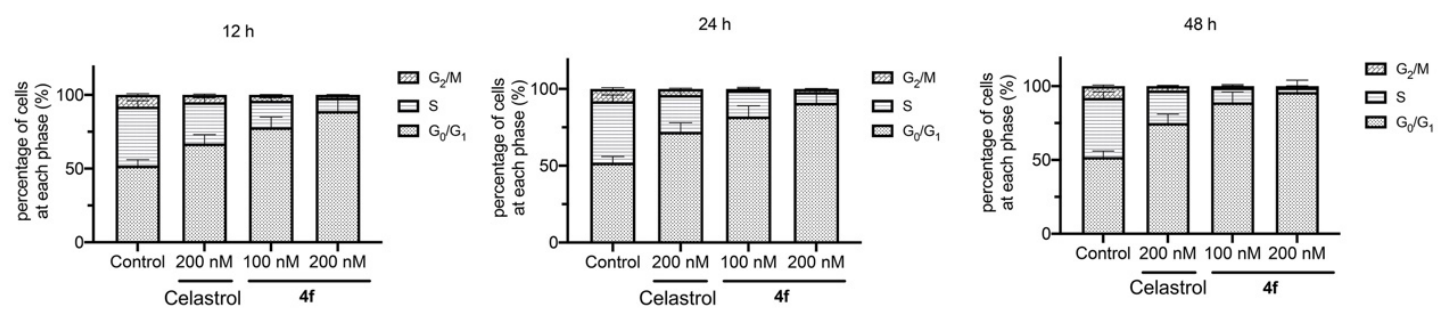

Figure S1. Cell cycle analysis of celastrol and $\mathbf{4 f}$ in MDA-MB-231 cells. Cells were treated with $\mathbf{4 f}$ at 100 and $200 \mathrm{nM}$ or CEL at $200 \mathrm{nM}$ for 0, 12, 24, and $48 \mathrm{~h}$. Cells were harvested and stained with PI, and then were analyzed by flow cytometry. The bar graphs were representative of three independent experiments and the values were expressed as means \pm S.D. 
A

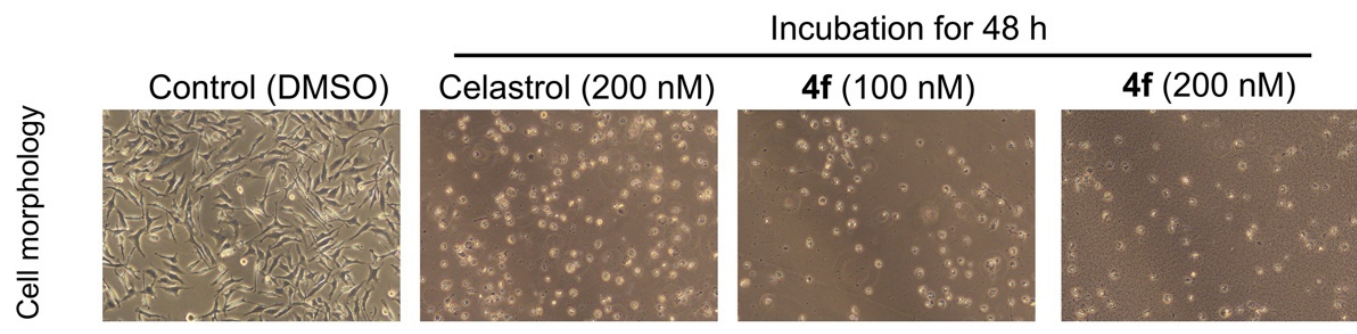

$\mathrm{B}$
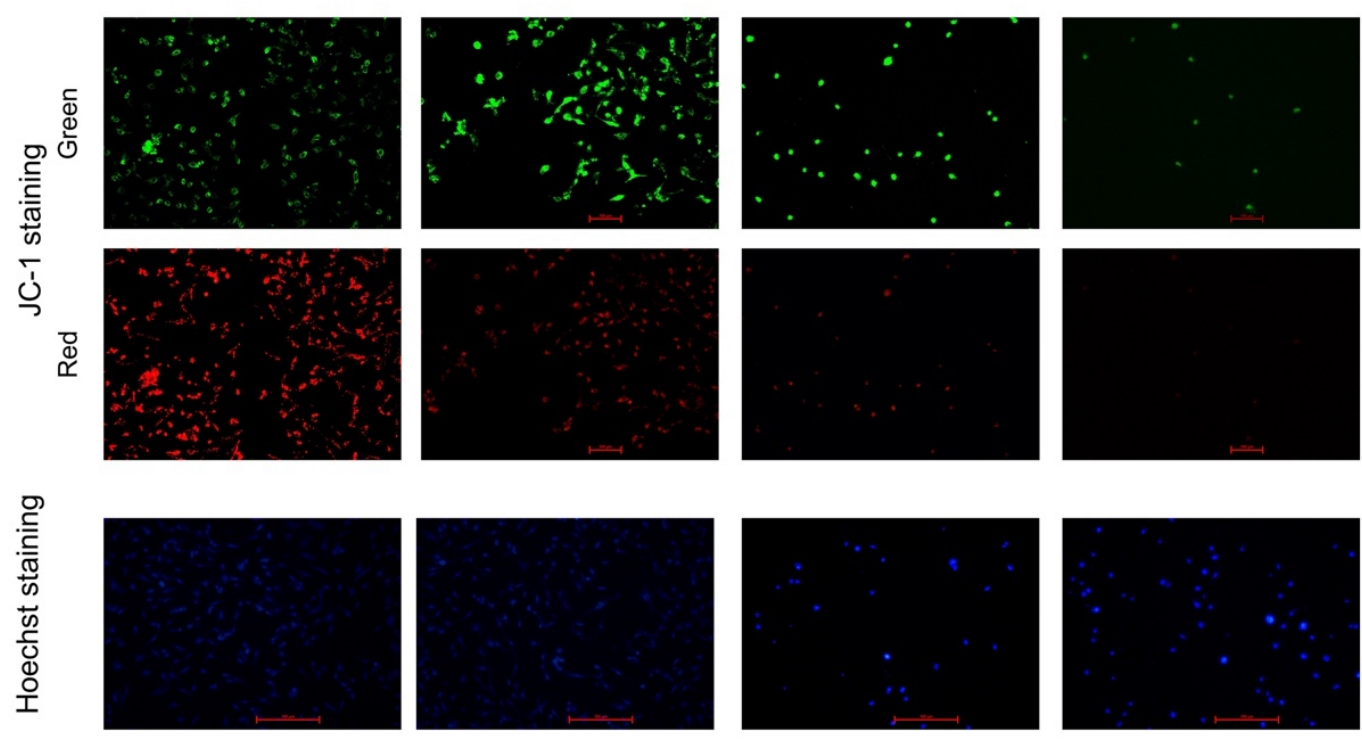

D
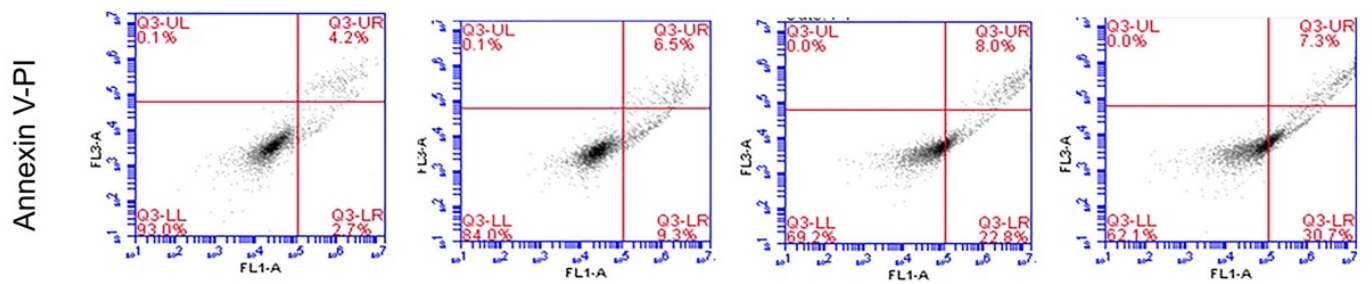

Figure S2. Apoptotic analysis of celastrol and $\mathbf{4 f}$ in MDA-MB-231 cells. Cells were treated with $\mathbf{4 f}$ at 100 and $200 \mathrm{nM}$ or celastrol at $200 \mathrm{nM}$ for $48 \mathrm{~h}$. (A) Cell morphology. (B) JC-1 staining for mitochondrial membrane potential (MMP was positively correlated with red fluorescence intensity). (C) Hoechst 33258 staining. (D) Annexin V-PI double staining, viable cells on the lower left, Annexin V-/PI-; necrotic cells on the upper left, Annexin $\mathrm{V}^{-} / \mathrm{PI}^{+}$; late apoptotic cells on the upper right, Annexin $\mathrm{V}^{+} / \mathrm{PI}^{+}$and early apoptotic cells on the lower right, Annexin $\mathrm{V}^{+} / \mathrm{PI}^{-}$. 


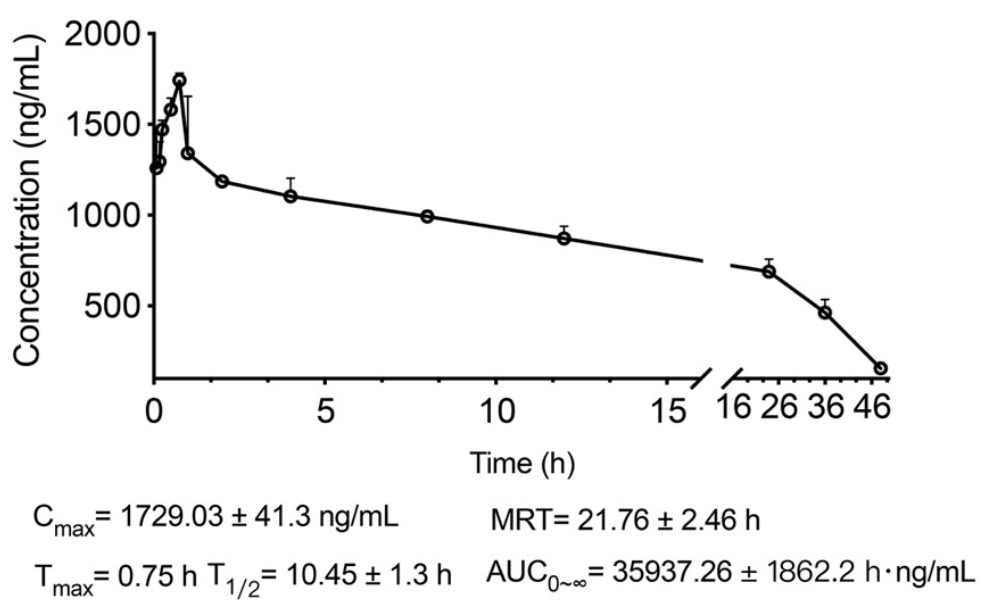

Figure S3. Concentration-time curves and PK parameters. Plasma concentration-time profile was evaluated after p.o. (10 $\mathrm{mg} / \mathrm{kg})$ administration of $\mathbf{4 f}$ to male SD rats (means \pm S.D., $n=6)$.

A
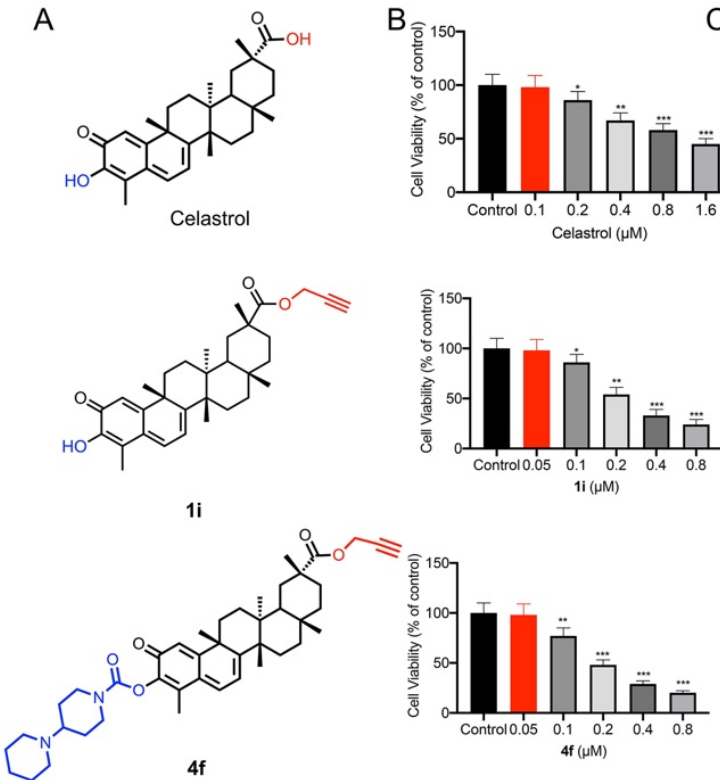

C
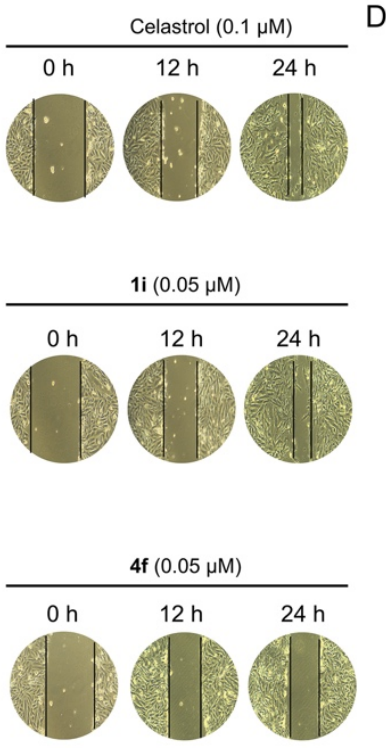
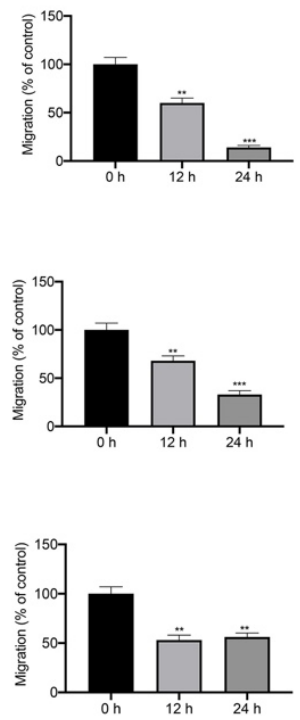

Figure S4. Migratory effects of celastrol, 1i, and $\mathbf{4 f}$ on HUVEC cells. (A) Chemical structures of celastrol, 1i, and $\mathbf{4 f}$. (B) Cell viability assay for the detecting of no-toxiceffect dose. (C) Effects of test compounds on migration of HUVECs cells. (D) The quantitative analysis of test compounds on migration of HUVECs cells. ${ }^{* *} p<0.01$ or ${ }^{* * *} p<0.001$ compared with $0 \mathrm{~h}$ group. 


\section{Supplementary Methods}

Caspase-3 and Caspase-9 Activities. MDA-MB-231 cells $\left(2 \times 10^{5}\right.$ cells/well $)$ were seeded into a 6-well plate and incubated at $37^{\circ} \mathrm{C}$ for $24 \mathrm{~h}$. Then cells were treated with various concentration of test compounds for $48 \mathrm{~h}$. After cell collection, the activities of caspase-3 and caspase-9 were determined following a previous report using the commercial kit.

Cell Cycle Assay. Cell-cycle distribution was detected by flow cytometry using a Fluorescence-Activated Cell Sorter (FACS). MDA-MB-231 cells $\left(2 \times 10^{5}\right.$ cells/well) were seeded into a 6-well plate allowed to culture for $24 \mathrm{~h}$. Then cells were treated for $48 \mathrm{~h}$ and with DMSO (blank control) or $2 \mathrm{f}$ (100 and $200 \mathrm{nM}$ ). Finally, cells were analyzed with a flow cytometry (BD Accuri C6, Becton \& Dickinson Company) according to the specification of the propidium iodide (PI) staining kit.

Hoechst33258 Staining and JC-1 Staining. After treating cells in the same way as above, the culture medium was removed, and cells were washed with cold PBS twice. Then, cells were stained with commercial Hoechst 33258 kit and JC-1 solution (KeyGEN, China) kit according to the specifications. The represented pictures were observed from a fluorescence microscope (Nikon TS-200).

Pharmacokinetic Analysis. Male SD rats weighing 220-250 g (Model Animal Center of Nanjing University) were intragastrically administered compounds $\mathbf{4 f}$ each at a single dose of $10 \mathrm{mg} / \mathrm{kg}$ after a $16 \mathrm{~h}$ fast. Then blood samples were collected at $0.083,0.167$, $0.25,0.5,0.75,1,2,4,8,12,24,36$, and $48 \mathrm{~h}$ after dosing. The plasma was prepared by centrifuging the blood at $5000 \mathrm{rpm}$ for $10 \mathrm{~min}$ at $4{ }^{\circ} \mathrm{C}$. Then, plasma samples were 
performed by mixing $150 \mu \mathrm{L}$ of plasma containing internal standard (compound 4a, $100 \mathrm{ng}$ ) standards solution with $3 \mathrm{~mL}$ of ethyl acetate, followed by $3 \mathrm{~min}$ vortex. Then, the samples were centrifuged for $10 \mathrm{~min}$ at $10,000 \times \mathrm{g}$ at $4{ }^{\circ} \mathrm{C}$. The ethyl acetate layer was then evaporated to dryness under $\mathrm{N}_{2}$ gas and reconstituted with $100 \mu \mathrm{L}$ of methanol. Then, a $5 \mu \mathrm{L}$ aliquot of the sample was injected into a Shimadzu 2020 LCMS single quadrupole system (Shimadzu Corp., Japan). Chromatographic separations were performed on an Agilent SB-C18 column $(4.6 \times 50 \mathrm{~mm})$. For the detection of compound 4f, an isocratic chromatographic procedure was used with a mobile phase of $70 \%$ acetonitrile and $30 \%$ water $\left(\mathrm{H}_{2} \mathrm{O}\right)$ at a flow rate of $0.3 \mathrm{~mL} / \mathrm{min}$. 


\section{Chemical names, yields, and spectral data of 2a-2d.}

prop-2-yn-1-yl $\quad(2 R, 4 a S, 6 a S, 12 b R, 14 a S)-10$-acetoxy-2,4a,6a, 9, 12b,14ahexamethyl-11-oxo-1,2,3,4,4a,5,6,6a,11,12b,13,14,14a,14b-tetradecahydropicene-2carboxylate (2a): Yellow solid; yield 51\%; mp $106-107^{\circ} \mathrm{C} ;[\alpha]_{\mathrm{D}}^{24}=+26.3(c=0.5$ in $\left.\mathrm{CHCl}_{3}\right) ;{ }^{1} \mathrm{H} \mathrm{NMR}\left(600 \mathrm{MHz}, \mathrm{CDCl}_{3}\right), \delta_{\mathrm{H}} 7.04(1 \mathrm{H}, \mathrm{d}, J=7.1 \mathrm{~Hz}, \mathrm{H}-6), 6.46(1 \mathrm{H}, \mathrm{s}, \mathrm{H}-$ 1), $6.27(1 \mathrm{H}, \mathrm{d}, J=7.1 \mathrm{~Hz}, \mathrm{H}-7), 4.48-4.65\left(2 \mathrm{H}, \mathrm{m}, \mathrm{H}-1^{\prime}\right), 2.44\left(1 \mathrm{H}, \mathrm{s}, \mathrm{C} \equiv \underline{\mathrm{CH}}, \mathrm{H}-3^{\prime}\right)$, $2.34(3 \mathrm{H}, \mathrm{s}, \mathrm{H}-2 "), 2.14$ (3H, s, H-23), $1.46(3 \mathrm{H}, \mathrm{s}, \mathrm{H}-25), 1.26(3 \mathrm{H}, \mathrm{s}, \mathrm{H}-26), 1.19(3 \mathrm{H}$, s, H-30), 1.09 (3H, s, H-28), 0.57 (3H, s, H-27); ${ }^{13} \mathrm{C} \mathrm{NMR} \mathrm{(150} \mathrm{MHz,} \mathrm{CDCl} 3$ ), $\delta_{\mathrm{C}} 177.7$, $177.4,172.0,168.8,163.3,143.1,135.6,133.8,126.4,123.0,117.9,77.5,75.2,52.0$, $45.4,44.3,42.8,40.5,39.4,38.4,36.4,34.7,33.7,32.6,31.7,30.7,30.6,29.9,29.6$, 28.7, 22.0, 20.6, 18.9, 11.4; HRMS (ESI) calculated for $\mathrm{C}_{34} \mathrm{H}_{43} \mathrm{O}_{5}[\mathrm{M}+\mathrm{H}]^{+}$531.3066, found 531.3032; purity 99.33\% $\left(\mathrm{CH}_{3} \mathrm{CN} / \mathrm{H}_{2} \mathrm{O}=90 / 10 ; \mathrm{R}_{\mathrm{t}}=13.834 \mathrm{~min}\right)$.

prop-2-yn-1-yl $\quad(2 R, 4 a S, 6 a S, 12 b R, 14 a S)-10$-(butyryloxy)-2,4a,6a,9,12b,14ahexamethyl-11-oxo-1,2,3,4,4a,5,6,6a,11,12b,13,14,14a,14b-tetradecahydropicene-2-

carboxylate (2b): Yellow solid; yield 51\%; mp 107-109 ${ }^{\circ} \mathrm{C} ;[\alpha]_{\mathrm{D}}^{24}+14.5(c=0.5$ in $\left.\mathrm{CHCl}_{3}\right) ;{ }^{1} \mathrm{H}$ NMR $\left(400 \mathrm{MHz}, \mathrm{CDCl}_{3}\right), \delta_{\mathrm{H}} 7.04(1 \mathrm{H}, \mathrm{d}, J=8.0 \mathrm{~Hz}, \mathrm{H}-6), 6.40(1 \mathrm{H}, \mathrm{s}, \mathrm{H}-$ 1), $6.32(1 \mathrm{H}, \mathrm{d}, J=8.0 \mathrm{~Hz}, \mathrm{H}-7), 4.48-4.67(2 \mathrm{H}, \mathrm{m}, \mathrm{H}-1$ '), $2.44(1 \mathrm{H}, \mathrm{s}, \mathrm{H}-3$ '), $2.13(3 \mathrm{H}$, s, H-23), 1.46 (3H, s, H-25), 1.26 (3H, s, H-26), 1.20 (3H, s, H-30), 1.09 (3H, s, H-28), 0.57 (3H, s, H-27); ${ }^{13} \mathrm{C}$ NMR (100 MHz, $\left.\mathrm{CDCl}_{3}\right), \delta_{\mathrm{C}} 177.8,177.5,171.9,171.6,163.3$, $143.2,135.6,133.8,126.6,123.1,118.0,77.7,75.3,52.2,45.4,44.4,42.9,40.6,39.5$, $38.5,36.5,36.0,34.8,33.8,32.8,31.8,30.8,30.7,30.1,29.8,28.8,22.1,19.0,18.8$, 13.9, 11.5; HRMS (ESI) calculated for $\mathrm{C}_{36} \mathrm{H}_{47} \mathrm{O}_{5}[\mathrm{M}+\mathrm{H}]^{+}$559.3379, found 559.3436; 
purity $99.82 \%\left(\mathrm{CH}_{3} \mathrm{CN} / \mathrm{H}_{2} \mathrm{O}=90 / 10 ; \mathrm{R}_{\mathrm{t}}=13.712 \mathrm{~min}\right)$.

prop-2-yn-1-yl (2R,4aS, 6aS, 12bR,14aS)-10-((4-bromobenzoyl)oxy)2,4a,6a,9,12b,14a-hexamethyl-11-oxo-1,2,3,4,4a,5,6,6a,11,12b,13,14,14a,14b-

tetradecahydropicene-2-carboxylate (2c) : Yellow solid; yield 51\%; mp 136-137 ${ }^{\circ} \mathrm{C}$; $[\alpha]_{\mathrm{D}}^{24}+42.1\left(c=0.5\right.$ in $\left.\mathrm{CHCl}_{3}\right) ;{ }^{1} \mathrm{H} \mathrm{NMR}\left(400 \mathrm{MHz}, \mathrm{CDCl}_{3}\right), \delta_{\mathrm{H}} 8.05(2 \mathrm{H}, \mathrm{d}, J=7.1 \mathrm{~Hz}$, Ar-H), 7.64 (2H, d, J= 7.1 Hz, Ar-H), 7.09 (1H, d, J=7.1 Hz, H-6), 6.51 (1H, s, H-1),

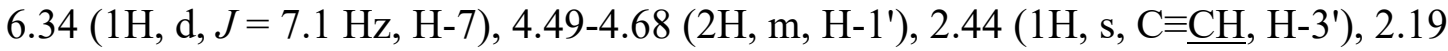
(3H, s, H-23), 1.49 (3H, s, H-25), 1.27 (3H, s, H-26), 1.21 (3H, s, H-30), $1.10(3 \mathrm{H}, \mathrm{s}$, $\mathrm{H}-28), 0.60(3 \mathrm{H}, \mathrm{s}, \mathrm{H}-27) ;{ }^{13} \mathrm{C} \mathrm{NMR}\left(100 \mathrm{MHz}, \mathrm{CDCl}_{3}\right), \delta_{\mathrm{C}} 177.6,177.5,172.3,167.4$, $163.9,163.5,143.2,135.8,132.2(2 \times \mathrm{C}), 132.1(2 \times \mathrm{C}), 128.9,128.3,126.6,123.2$, $118.1,77.7,75.3,52.2,45.5,44.5,43.0,40.6,39.6,38.5,36.5,34.8,33.8,32.8,31.8$, $30.8,30.7,30.1,29.8,28.9,22.2,19.0,11.7$; HRMS (ESI) calculated for $\mathrm{C}_{39} \mathrm{H}_{44} \mathrm{BrO}_{5}$ $[\mathrm{M}+\mathrm{H}]^{+} 671.2429$, found $671.2402 ;$ purity $99.32 \%\left(\mathrm{CH}_{3} \mathrm{CN} / \mathrm{H}_{2} \mathrm{O}=90 / 10 ; \mathrm{R}_{\mathrm{t}}=13.465\right.$ $\min )$.

prop-2-yn-1-yl $\quad(2 R, 4 a S, 6 a S, 12 b R, 14 a S)$-10-((4-fluorobenzoyl)oxy)2,4a,6a,9,12b,14a-hexamethyl-11-oxo-1,2,3,4,4a,5,6,6a,11,12b,13,14,14a,14btetradecahydropicene-2-carboxylate (2d): Yellow solid; yield 51\%; mp 204-206 ${ }^{\circ} \mathrm{C}$; $[\alpha]_{\mathrm{D}}^{24}+38.4\left(c=0.5\right.$ in $\left.\mathrm{CHCl}_{3}\right) ;{ }^{1} \mathrm{H}$ NMR $\left(600 \mathrm{MHz}, \mathrm{CDCl}_{3}\right), \delta_{\mathrm{H}} 8.22(2 \mathrm{H}, \mathrm{m}, \mathrm{Ar}-\mathrm{H})$, $7.15(2 \mathrm{H}, \mathrm{m}, \mathrm{Ar}-\mathrm{H}), 7.08(1 \mathrm{H}, \mathrm{d}, J=7.1 \mathrm{~Hz}, \mathrm{H}-6), 6.51(1 \mathrm{H}, \mathrm{s}, \mathrm{H}-1), 6.35(1 \mathrm{H}, \mathrm{d}, J=$ $7.1 \mathrm{~Hz}, \mathrm{H}-7), 4.50-4.67$ (2H, m, H-1'), 2.44 (1H, s, C $\equiv \underline{\mathrm{CH}}, \mathrm{H}-3$ '), 2.19 (3H, s, H-23), 1.49 (3H, s, H-25), 1.27 (3H, s, H-26), 1.21 (3H, s, H-30), 1.10 (3H, s, H-28), 0.60 (3H, s, H-27); ${ }^{13} \mathrm{C} \mathrm{NMR}\left(150 \mathrm{MHz}, \mathrm{CDCl}_{3}\right), \delta_{\mathrm{C}} 177.7,177.5,172.2,166.4(\mathrm{~d}, J=253.5 \mathrm{~Hz})$, 
163.6, 163.4, 143.3, 135.8,134.1, $133.3(2 \times \mathrm{C}, \mathrm{d}, J=9.0 \mathrm{~Hz}), 126.6,125.6(\mathrm{~d}, J=3.0$ $\mathrm{Hz}), 123.2,118.1,115.9(2 \times \mathrm{C}, \mathrm{d}, J=22.5 \mathrm{~Hz}), 77.7,75.3,52.2,45.5,44.5,43.0,40.6$, $39.6,38.5,36.5,34.9,33.8,32.8,31.8,30.8,30.7,30.1,29.8,28.9,22.2,19.0,11.7$; HRMS (ESI) calculated for $\mathrm{C}_{39} \mathrm{H}_{44} \mathrm{FO}_{5}[\mathrm{M}+\mathrm{H}]^{+}$611.3128, found 611.3198; purity $97.02 \%\left(\mathrm{CH}_{3} \mathrm{CN} / \mathrm{H}_{2} \mathrm{O}=90 / 10 ; \mathrm{R}_{\mathrm{t}}=14.488 \mathrm{~min}\right)$.

\section{Chemical names, yields, and spectral data of 3a-3g.}

prop-2-yn-1-yl $\quad(2 R, 4 a S, 6 a S, 12 b R, 14 a S)$-10-(acryloyloxy)-2, 4a, 6a, 9, 12b,14ahexamethyl-11-oxo-1,2,3,4,4a,5,6,6a,11,12b,13,14,14a,14b-tetradecahydropicene-2-

carboxylate (3a): Yellow solid; yield 51\%; mp $121-122{ }^{\circ} \mathrm{C} ;[\alpha]_{\mathrm{D}}^{24}+48.7(c=0.5$ in $\left.\mathrm{CHCl}_{3}\right) ;{ }^{1} \mathrm{H} \mathrm{NMR}\left(600 \mathrm{MHz}, \mathrm{CDCl}_{3}\right), \delta_{\mathrm{H}} 7.05(1 \mathrm{H}, \mathrm{d}, J=7.1 \mathrm{~Hz}, \mathrm{H}-6), 6.61(1 \mathrm{H}, \mathrm{d}, J$ $=18.0 \mathrm{~Hz}, \mathrm{H}-3 "$ ), 6.48 (1H, s, H-1), 6.31 (1H, m, H-3"), $6.32(1 \mathrm{H}, \mathrm{d}, J=7.1 \mathrm{~Hz}, \mathrm{H}-7)$, $6.00\left(1 \mathrm{H}, \mathrm{d}, J=18.0 \mathrm{~Hz}, \mathrm{H}-2^{\prime \prime}\right), 4.48-4.65\left(2 \mathrm{H}, \mathrm{m}, \mathrm{H}-1^{\prime}\right), 2.43\left(1 \mathrm{H}, \mathrm{s}, \mathrm{C} \equiv \underline{\mathrm{CH}}, \mathrm{H}-3^{\prime}\right)$, $2.14(3 \mathrm{H}, \mathrm{s}, \mathrm{H}-23), 1.46$ (3H, s, H-25), 1.25 (3H, s, H-26), 1.19 (3H, s, H-30), $1.08(3 \mathrm{H}$, s, H-28), 0.57 (3H, s, H-27); ${ }^{13} \mathrm{C}$ NMR (150 MHz, $\left.\mathrm{CDCl}_{3}\right), \delta_{\mathrm{C}} 177.6,177.5,172.0$, $163.8,163.3,142.9,135.7,134.0,133.0,127.5,126.5,123.1,118.0,77.6,75.3,52.1$, $45.4,44.4,42.9,40.6,39.5,38.5,36.5,34.8,33.8,32.7,31.8,30.8,30.7,30.0,29.7$, 28.8, 22.1, 19.0, 11.6; HRMS (ESI) calculated for $\mathrm{C}_{35} \mathrm{H}_{43} \mathrm{O}_{5}[\mathrm{M}+\mathrm{H}]^{+}$543.3136, found 543.3132; purity 95.24\% $\left(\mathrm{CH}_{3} \mathrm{CN} / \mathrm{H}_{2} \mathrm{O}=90 / 10 ; \mathrm{R}_{\mathrm{t}}=10.766 \mathrm{~min}\right)$.

$$
\text { prop-2-yn-1-yl } \quad(2 R, 4 a S, 6 a S, 12 b R, 14 a S)-10-(((E)-b u t-2-e n o y l) \text { oxy }) \text { - }
$$

2,4a,6a,9,12b,14a-hexamethyl-11-oxo-1,2,3,4,4a,5,6,6a,11,12b,13,14,14a,14b-

tetradecahydropicene-2-carboxylate (3b): Yellow solid; yield 51\%; mp 103-105 ${ }^{\circ} \mathrm{C}$; $[\alpha]_{\mathrm{D}}^{24}+64.2\left(c=0.5\right.$ in $\left.\mathrm{CHCl}_{3}\right) ;{ }^{1} \mathrm{H} \mathrm{NMR}\left(400 \mathrm{MHz}, \mathrm{CDCl}_{3}\right), \delta_{\mathrm{H}} 7.18(1 \mathrm{H}, \mathrm{d}, J=16.0$ 
Hz, H-3"), 7.05 (1H, d, J=7.1 Hz, H-6), $6.48(1 \mathrm{H}, \mathrm{s}, \mathrm{H}-1), 6.32(1 \mathrm{H}, \mathrm{d}, J=7.1 \mathrm{~Hz}, \mathrm{H}-$ 7), $6.09\left(1 \mathrm{H}, J=16.0 \mathrm{~Hz}, \mathrm{H}-2^{\prime \prime}\right), 4.48-4.65\left(2 \mathrm{H}, \mathrm{m}, \mathrm{H}-1^{\prime}\right), 2.43\left(1 \mathrm{H}, \mathrm{s}, \mathrm{C} \equiv \underline{\mathrm{CH}}, \mathrm{H}-3^{\prime}\right)$, $2.13(3 \mathrm{H}, \mathrm{s}, \mathrm{H}-23), 1.46$ (3H, s, H-25), 1.25 (3H, s, H-26), 1.19 (3H, s, H-30), 1.08 (3H, s, H-28), 0.57 (3H, s, H-27); ${ }^{13} \mathrm{C}$ NMR (100 MHz, $\left.\mathrm{CDCl}_{3}\right), \delta_{\mathrm{C}} 177.9,177.5,171.9$, $164.1,163.3,147.5,143.0,135.5,134.0,126.6,123.2,121.7,118.0,77.6,75.3,52.1$, $45.4,44.4,42.9,40.6,39.5,38.5,36.5,34.8,33.8,32.7,31.8,30.8,30.7,30.0,29.8$, 28.8, 22.1, 19.0, 18.4, 11.6; HRMS (ESI) calculated for $\mathrm{C}_{36} \mathrm{H}_{45} \mathrm{O}_{5}[\mathrm{M}+\mathrm{H}]^{+}$557.3222, found 557.3289; purity 97.67\% $\left(\mathrm{CH}_{3} \mathrm{CN} / \mathrm{H}_{2} \mathrm{O}=90 / 10 ; \mathrm{R}_{\mathrm{t}}=12.064 \mathrm{~min}\right)$.

prop-2-yn-1-yl $\quad(2 R, 4 a S, 6 a S, 12 b R, 14 a S)-10-($ cinnamoyloxy)-2,4a, 6a, 9, 12b,14ahexamethyl-11-oxo-1,2,3,4,4a,5,6,6a,11,12b,13,14,14a,14b-tetradecahydropicene-2-

carboxylate (3c): Yellow solid; yield 51\%; mp $118-119^{\circ} \mathrm{C} ;[\alpha]_{\mathrm{D}}^{24}+70.5(c=0.5$ in $\left.\mathrm{CHCl}_{3}\right) ;{ }^{1} \mathrm{H}$ NMR $\left(600 \mathrm{MHz}, \mathrm{CDCl}_{3}\right), \delta_{\mathrm{H}} 7.87(1 \mathrm{H}, \mathrm{d}, J=18.0 \mathrm{~Hz}, \mathrm{H}-3 "), 7.39-7.59$ (5H, m, Ar-H), $7.08(1 \mathrm{H}, \mathrm{d}, J=7.1 \mathrm{~Hz}, \mathrm{H}-6), 6.68(1 \mathrm{H}, \mathrm{d}, J=18.0 \mathrm{~Hz}, \mathrm{H}-2 "), 6.53(1 \mathrm{H}$, s, H-1), 6.34 (1H, d, J= 7.1 Hz, H-7), 4.49-4.67 (2H, m, H-1'), $2.43(1 \mathrm{H}, \mathrm{s}, \mathrm{C} \equiv \underline{\mathrm{CH}}, \mathrm{H}-$ 3'), 2.19 (3H, s, H-23), 1.48 (3H, s, H-25), 1.26 (3H, s, H-26), 1.20 (3H, s, H-30), 1.09 (3H, s, H-28), 0.58 (3H, s, H-27); ${ }^{13} \mathrm{C}$ NMR (150 MHz, $\left.\mathrm{CDCl}_{3}\right), \delta_{\mathrm{C}} 177.9,177.5,172.1$, $164.7,163.4,147.1,146.4,143.1,135.8,134.5,134.2,130.8,129.1,128.5,128.4,126.6$, $123.2,118.1,117.0,77.6,75.3,52.1,45.5,44.4,42.9,40.6,39.5,38.5,36.5,34.8,33.8$, 32.7, 31.8, 30.8, 30.7, 30.0, 29.8, 28.8, 22.1, 19.0, 11.7; HRMS (ESI) calculated for $\mathrm{C}_{41} \mathrm{H}_{47} \mathrm{O}_{5}[\mathrm{M}+\mathrm{H}]^{+}$619.3379, found 619.3450; purity 97.52\% $\left(\mathrm{CH}_{3} \mathrm{CN} / \mathrm{H}_{2} \mathrm{O}=90 / 10\right.$; $\left.\mathrm{R}_{\mathrm{t}}=15.586 \mathrm{~min}\right)$. 
fluorophenyl)acryloyl)oxy)-2,4a,6a,9,12b,14a-hexamethyl-11-oxo-

Yellow solid; yield 51\%; mp $108-109^{\circ} \mathrm{C} ;[\alpha]_{\mathrm{D}}^{24}+70.7\left(c=0.5\right.$ in $\left.\mathrm{CHCl}_{3}\right) ;{ }^{1} \mathrm{H}$ NMR $(600$ $\left.\mathrm{MHz}, \mathrm{CDCl}_{3}\right), \delta_{\mathrm{H}} 7.98(1 \mathrm{H}, \mathrm{d}, J=18.0 \mathrm{~Hz}, \mathrm{H}-3 "), 7.58(1 \mathrm{H}, \mathrm{m}, \mathrm{Ar}-\mathrm{H}), 7.37(1 \mathrm{H}, \mathrm{m}$, Ar-H), $7.16(1 \mathrm{H}, \mathrm{m}, \mathrm{Ar}-\mathrm{H}), 7.12(1 \mathrm{H}, \mathrm{m}, \mathrm{Ar}-\mathrm{H}), 7.08(1 \mathrm{H}, \mathrm{d}, J=7.1 \mathrm{~Hz}, \mathrm{H}-6), 6.78$ $(1 \mathrm{H}, \mathrm{d}, J=18.0 \mathrm{~Hz}, \mathrm{H}-2 "), 6.50(1 \mathrm{H}, \mathrm{s}, \mathrm{H}-1), 6.32(1 \mathrm{H}, \mathrm{d}, J=7.1 \mathrm{~Hz}, \mathrm{H}-7), 4.48-4.65$ (2H, m, H-1'), $2.43\left(1 \mathrm{H}, \mathrm{s}, \mathrm{C} \equiv \mathrm{CH}, \mathrm{H}-3^{\prime}\right), 2.18(3 \mathrm{H}, \mathrm{s}, \mathrm{H}-23), 1.47$ (3H, s, H-25), 1.25 (3H, s, H-26), 1.19 (3H, s, H-30), 1.08 (3H, s, H-28), 0.57 (3H, s, H-27); ${ }^{13} \mathrm{C}$ NMR (150 $\left.\mathrm{MHz}, \mathrm{CDCl}_{3}\right), \delta_{\mathrm{C}} 177.6,177.3,172.0,164.4,163.2,161.6(\mathrm{~d}, J=253.5 \mathrm{~Hz}), 142.9$, 139.4, 135.7 (d, $J=7.5 \mathrm{~Hz}), 134.0,132.1$ (d, $J=9.0 \mathrm{~Hz}), 129.4,126.4$, 124.6, 123.0, $122.5(\mathrm{~d}, J=12.0 \mathrm{~Hz}), 119.4(\mathrm{~d}, J=6.0 \mathrm{~Hz}), 117.9,116.3(\mathrm{~d}, J=22.5 \mathrm{~Hz}), 77.5,75.2$, $52.0,45.3,44.3,42.8,40.4,39.4,38.3,36.4,34.7,33.6,32.6,31.6,30.6,30.6,29.9$, 29.6, 28.7, 22.0, 18.9, 11.5; HRMS (ESI) calculated for $\mathrm{C}_{41} \mathrm{H}_{46} \mathrm{FO}_{5}[\mathrm{M}+\mathrm{H}]^{+}$637.3285, found 637.3357; purity $97.61 \%\left(\mathrm{CH}_{3} \mathrm{CN} / \mathrm{H}_{2} \mathrm{O}=90 / 10 ; \mathrm{R}_{\mathrm{t}}=16.455 \mathrm{~min}\right)$.

prop-2-yn-1-yl $\quad(2 R, 4 a S, 6 a S, 12 b R, 14 a S)-10-(((E)-3-(3-$ chlorophenyl)acryloyl)oxy)-2,4a,6a,9,12b,14a-hexamethyl-11-oxo1,2,3,4,4a,5,6,6a,11,12b,13,14,14a,14b-tetradecahydropicene-2-carboxylate (3e): Yellow solid; yield 51\%; mp $119-120{ }^{\circ} \mathrm{C} ;[\alpha]_{\mathrm{D}}^{24}+77.9\left(c=0.5\right.$ in $\left.\mathrm{CHCl}_{3}\right) ;{ }^{1} \mathrm{H}$ NMR $(600$ $\left.\mathrm{MHz}, \mathrm{CDCl}_{3}\right), \delta_{\mathrm{H}} 7.76(1 \mathrm{H}, \mathrm{d}, J=18.0 \mathrm{~Hz}, \mathrm{H}-3 "), 7.53(1 \mathrm{H}, \mathrm{m}, \mathrm{Ar}-\mathrm{H}), 7.42(1 \mathrm{H}, \mathrm{m}$, Ar-H), 7.30-7.35 (2H, m, Ar-H), $7.06(1 \mathrm{H}, \mathrm{d}, J=7.1 \mathrm{~Hz}, \mathrm{H}-6), 6.66(1 \mathrm{H}, \mathrm{d}, J=18.0$ Hz, H-2"), 6.48 (1H, s, H-1), 6.32 (1H, d, $J=7.1 \mathrm{~Hz}, \mathrm{H}-7), 4.47-4.64$ (2H, m, H-1'), $2.43\left(1 \mathrm{H}, \mathrm{s}, \mathrm{C} \equiv \mathrm{CH}, \mathrm{H}-3^{\prime}\right), 2.18$ (3H, s, H-23), 1.46 (3H, s, H-25), 1.25 (3H, s, H-26), 
1.19 (3H, s, H-30), 1.08 (3H, s, H-28), 0.57 (3H, s, H-27); ${ }^{13} \mathrm{C} \mathrm{NMR}\left(150 \mathrm{MHz}, \mathrm{CDCl}_{3}\right)$, $\delta_{\mathrm{C}} 177.6,177.3,172.0,164.4,163.2,142.9,139.4,135.6,134.0,132.1,129.4,126.4$, $124.6,123.0,122.5,119.4,117.9,116.4,116.2,77.5,75.2,52.0,45.3,44.3,42.8,40.4$, $39.4,38.3,36.4,34.7,33.6,32.6,31.6,30.6,30.6,29.9,29.6,28.7,22.0,18.9,11.5$; HRMS (ESI) calculated for $\mathrm{C}_{41} \mathrm{H}_{46} \mathrm{ClO}_{5}[\mathrm{M}+\mathrm{H}]^{+}$653.2989, found 653.3063; purity $97.01 \%\left(\mathrm{CH}_{3} \mathrm{CN} / \mathrm{H}_{2} \mathrm{O}=90 / 10 ; \mathrm{R}_{\mathrm{t}}=14.834 \mathrm{~min}\right)$.

prop-2-yn-1-yl $\quad(2 R, 4 a S, 6 a S, 12 b R, 14 a S)-2,4 a, 6 a, 9,12 b, 14 a$-hexamethyl-11-oxo10-(((E)-3-(m-tolyl)acryloyl)oxy)-1,2,3,4,4a,5,6,6a,11,12b,13,14,14a,14b-

tetradecahydropicene-2-carboxylate (3f): Yellow solid; yield 51\%; mp 95-97 ${ }^{\circ} \mathrm{C} ;[\alpha]$ ${ }_{\mathrm{D}}^{24}+79.8\left(c=0.5\right.$ in $\left.\mathrm{CHCl}_{3}\right) ;{ }^{1} \mathrm{H} \mathrm{NMR}\left(400 \mathrm{MHz}, \mathrm{CDCl}_{3}\right), \delta_{\mathrm{H}} 7.84(1 \mathrm{H}, \mathrm{d}, J=16.0 \mathrm{~Hz}$, H-3"), 7.36-7.38 (2H, m, Ar-H), 7.29 (1H, d, J=16.0 Hz, H-2"), 7.21 (1H, m, Ar-H), $7.06(1 \mathrm{H}, \mathrm{d}, J=7.1 \mathrm{~Hz}, \mathrm{H}-6), 6.67(1 \mathrm{H}, \mathrm{m}, \mathrm{Ar}-\mathrm{H}), 6.50(1 \mathrm{H}, \mathrm{s}, \mathrm{H}-1), 6.32(1 \mathrm{H}, \mathrm{d}, J=$ $7.1 \mathrm{~Hz}, \mathrm{H}-7), 4.48-4.67$ (2H, m, H-1'), $2.44\left(1 \mathrm{H}, \mathrm{s}, \mathrm{C} \equiv \underline{\mathrm{CH}}, \mathrm{H}-3\right.$ '), 2.37 (3H, s, Ar- $\left.\underline{\mathrm{CH}}_{3}\right)$, 2.18 (3H, s, H-23), 1.48 (3H, s, H-25), 1.26 (3H, s, H-26), 1.20 (3H, s, H-30), 1.09 (3H, s, H-28), 0.59 (3H, s, H-27); ${ }^{13} \mathrm{C}$ NMR (100 MHz, $\left.\mathrm{CDCl}_{3}\right), \delta_{\mathrm{C}} 177.7,177.4,171.8$, $164.6,163.2,147.1,143.0,138.6,135.5,134.3,133.9,131.5,129.1,128.9,126.5,125.6$, $123.1,117.9,116.6,77.5,75.2,52.0,45.3,44.3,42.8,40.5,39.4,38.4,36.4,34.7,33.7$, 32.6, 31.7, 30.7, 30.6, 29.9, 29.6, 28.7, 22.0, 21.4, 18.9, 11.5; HRMS (ESI) calculated for $\mathrm{C}_{42} \mathrm{H}_{49} \mathrm{O}_{5}[\mathrm{M}+\mathrm{H}]^{+}$633.3535, found 633.3607; purity 98.79\% $\left(\mathrm{CH}_{3} \mathrm{CN} / \mathrm{H}_{2} \mathrm{O}=90 / 10\right.$; $\left.\mathrm{R}_{\mathrm{t}}=18.874 \mathrm{~min}\right)$.

prop-2-yn-1-yl $\quad(2 R, 4 a S, 6 a S, 12 b R, 14 a S)-10$-(methacryloyloxy)2,4a,6a,9,12b,14a-hexamethyl-11-oxo-1,2,3,4,4a,5,6,6a,11,12b,13,14,14a,14b- 
tetradecahydropicene-2-carboxylate (3g): Yellow solid; yield 51\%; mp 161-163 ${ }^{\circ} \mathrm{C}$; $[\alpha]_{\mathrm{D}}^{24}+76.2\left(c=0.5\right.$ in $\left.\mathrm{CHCl}_{3}\right) ;{ }^{1} \mathrm{H} \mathrm{NMR}\left(400 \mathrm{MHz}, \mathrm{CDCl}_{3}\right), \delta_{\mathrm{H}} 7.06(1 \mathrm{H}, \mathrm{d}, J=8.0 \mathrm{~Hz}$, H-6), $6.48(1 \mathrm{H}, \mathrm{s}, \mathrm{H}-1), 6.36(1 \mathrm{H}, \mathrm{s}, \mathrm{H}-3 "), 6.32(1 \mathrm{H}, \mathrm{d}, J=8.0 \mathrm{~Hz}, \mathrm{H}-7), 5.75$ (1H, s, H-3"), 4.48-4.67 (2H, m, H-1'), 2.44 (1H, s, CE $\underline{\mathrm{CH}}, \mathrm{H}-3$ '), 2.15 (3H, s, H-23), $2.05(3 \mathrm{H}$, s, $\left.\mathrm{CH}_{3}\right), 1.46(3 \mathrm{H}, \mathrm{s}, \mathrm{H}-25), 1.26(3 \mathrm{H}, \mathrm{s}, \mathrm{H}-26), 1.20$ (3H, s, H-30), 1.09 (3H, s, H-28), $0.58(3 \mathrm{H}, \mathrm{s}, \mathrm{H}-27) ;{ }^{13} \mathrm{C} \mathrm{NMR}\left(100 \mathrm{MHz}, \mathrm{CDCl}_{3}\right), \delta_{\mathrm{C}} 177.7,177.4,171.7,165.1,163.2$, $143.2,135.4,135.4,133.8,127.6,126.6,123.1,117.9,77.6,75.2,52.0,45.3,44.3,42.7$, $40.5,39.4,38.4,36.4,34.7,33.7,32.7,31.7,30.7,30.6,30.0,29.7,28.7,22.0,18.9$, 18.6, 11.4; HRMS (ESI) calculated for $\mathrm{C}_{36} \mathrm{H}_{45} \mathrm{O}_{5}[\mathrm{M}+\mathrm{H}]^{+}$557.3222, found 557.3289; purity $98.18 \%\left(\mathrm{CH}_{3} \mathrm{CN} / \mathrm{H}_{2} \mathrm{O}=90 / 10 ; \mathrm{R}_{\mathrm{t}}=12.775 \mathrm{~min}\right)$.

\section{Chemical names, yields, and spectral data of 4a-4t.}

Prop-2-yn-1-yl (2R,4aS,6aS, 12bR,14aS)-10-((diethylcarbamoyl)oxy)2,4a,6a,9,12b,14a-hexamethyl-11-oxo-1,2,3,4,4a,5,6,6a,11,12b,13,14,14a,14btetradecahydropicene-2-carboxylate (4a): Yellow solid; yield 51\%; mp 184-186 ${ }^{\circ} \mathrm{C}$; $[\alpha]_{\mathrm{D}}^{24}+55.8\left(c=0.5\right.$ in $\left.\mathrm{CHCl}_{3}\right) ; \quad{ }^{1} \mathrm{H} \mathrm{NMR}\left(600 \mathrm{MHz}, \mathrm{CDCl}_{3}\right), \delta_{\mathrm{H}} 6.99(1 \mathrm{H}, \mathrm{d}, J=7.1$ Hz, H-6), 6.41 (1H, s, H-1), 6.27 (1H, d, $J=7.1 \mathrm{~Hz}, \mathrm{H}-7), 4.46-4.64$ (2H, m, H-1'), $2.43(1 \mathrm{H}, \mathrm{s}, \mathrm{C} \equiv \mathrm{CH}, \mathrm{H}-3$ '), 3.36-3.48 (2H, m), $2.16(3 \mathrm{H}, \mathrm{s}, \mathrm{H}-23), 1.44(3 \mathrm{H}, \mathrm{s}, \mathrm{H}-25)$, 1.24 (3H, s, H-26), 1.19 (3H, s, H-30), 1.08 (3H, s, H-28), 0.56 (3H, s, H-27); ${ }^{13} \mathrm{C}$ NMR $\left(150 \mathrm{MHz}, \mathrm{CDCl}_{3}\right), \delta_{\mathrm{C}} 178.6,177.3,170.7,162.8,153.5,143.3,134.7,133.2,126.8$, $123.1,117.8,77.5,75.1,51.9,45.1,44.3,42.5,42.3,42.3,40.4,39.3,38.2,36.4,34.7$, 33.6, 32.6, 31.6, 30.6, 30.5, 29.9, 29.6, 28.7, 22.0, 18.8, 14.2, 13.4, 11.3; HRMS (ESI) calculated for $\mathrm{C}_{37} \mathrm{H}_{50} \mathrm{NO}_{5}[\mathrm{M}+\mathrm{H}]^{+}$588.3644, found 588.3691; purity $99.60 \%$ 
$\left(\mathrm{CH}_{3} \mathrm{CN} / \mathrm{H}_{2} \mathrm{O}=90 / 10 ; \mathrm{R}_{\mathrm{t}}=14.685 \mathrm{~min}\right)$.

Prop-2-yn-1-yl $\quad(2 R, 4 a S, 6 a S, 12 b R, 14 a S)-10-((e t h y l(m e t h y l)$ carbamoyl)oxy)2,4a,6a,9,12b,14a-hexamethyl-11-oxo-1,2,3,4,4a,5,6,6a,11,12b,13,14,14a,14btetradecahydropicene-2-carboxylate (4b): Yellow solid; yield 57\%; mp $122-124{ }^{\circ} \mathrm{C}$; $[\alpha]_{\mathrm{D}}^{24}+36.0\left(c=0.5\right.$ in $\left.\mathrm{CHCl}_{3}\right) ;{ }^{1} \mathrm{H} \mathrm{NMR}\left(600 \mathrm{MHz}, \mathrm{CDCl}_{3}\right), \delta_{\mathrm{H}} 7.00(1 \mathrm{H}, \mathrm{d}, J=7.1 \mathrm{~Hz}$, H-6), $6.43(1 \mathrm{H}, \mathrm{s}, \mathrm{H}-1), 6.28(1 \mathrm{H}, \mathrm{d}, J=7.1 \mathrm{~Hz}, \mathrm{H}-7), 4.47-4.63(2 \mathrm{H}, \mathrm{m}, \mathrm{H}-1$ '), 3.33-3.45 (4H, m), 2.43 (1H, s, C $\equiv \mathrm{CH}, \mathrm{H}-3$ '), 2.15 (3H, s, H-23), 1.42 (3H, s, H-25), 1.23 (3H, s, H-26), 1.18 (3H, s, H-26), 1.07 (3H, s, H-28), 0.55 (3H, s, H-27); ${ }^{13} \mathrm{C}$ NMR $\left(150 \mathrm{MHz}, \mathrm{CDCl}_{3}\right), \delta_{\mathrm{C}} 178.7,177.4,170.9,162.9,153.9,143.3,134.8,133.4,126.9$, $123.1,117.9,77.5,75.2,52.0,45.2,44.5,44.3,42.6,40.5,39.4,38.3,36.4,34.7,34.4$, 34.1,32.7, 31.7, 30.7, 30.6, 30.0, 29.7, 28.7, 22.0, 18.8, 13.2, 11.3; HRMS (ESI) calculated for $\mathrm{C}_{36} \mathrm{H}_{48} \mathrm{NO}_{5}[\mathrm{M}+\mathrm{H}]^{+} 574.3488$, found 574.3535 ; purity $99.62 \%$ $\left(\mathrm{CH}_{3} \mathrm{CN} / \mathrm{H}_{2} \mathrm{O}=90 / 10 ; \mathrm{R}_{\mathrm{t}}=12.369 \mathrm{~min}\right)$.

Prop-2-yn-1-yl $\quad(2 R, 4 a S, 6 a S, 12 b R, 14 a S)-10-(($ diphenylcarbamoyl)oxy)$2,4^{a}, 6^{a}, 9,12 b, 14^{a}$-hexamethyl-11-oxo-1,2,3,4,4 $4^{a}, 5,6,6^{a}, 11,12 b, 13,14,14^{a}, 14 b-$

tetradecahydropicene-2-carboxylate (4c): Yellow solid; yield 59\%; mp $161-162{ }^{\circ} \mathrm{C}$; $[\alpha]_{\mathrm{D}}^{24}+68.3\left(c=0.5\right.$ in $\left.\mathrm{CHCl}_{3}\right) ;{ }^{1} \mathrm{H} \mathrm{NMR}\left(600 \mathrm{MHz}, \mathrm{CDCl}_{3}\right), \delta_{\mathrm{H}}$ 7.19-7.46 (10H, m, ArH), $7.01(1 \mathrm{H}, \mathrm{d}, J=7.1 \mathrm{~Hz}, \mathrm{H}-6), 6.45(1 \mathrm{H}, \mathrm{s}, \mathrm{H}-1), 6.29(1 \mathrm{H}, \mathrm{d}, J=7.1 \mathrm{~Hz}, \mathrm{H}-7)$, 4.49-4.66 (2H, m, H-1'), 2.43 (1H, s, C $\equiv \mathrm{CH}, \mathrm{H}-3$ '), 2.16 (3H, s, H-23), 1.43 (3H, s, H25), 1.25 (3H, s, H-26), 1.20 (3H, s, H-30), 1.09 (3H, s, H-28), 0.57 (3H, s, H-27); ${ }^{13} \mathrm{C}$ NMR $\left(150 \mathrm{MHz}, \mathrm{CDCl}_{3}\right), \delta_{\mathrm{C}} 178.0,177.4,171.3,163.2,152.5,143.4,142.8(2 \times \mathrm{C})$, $135.2,133.3,129.0(8 \times C), 126.7,126.3(2 \times C), 123.0,117.9,77.5,75.2,52.0,45.3$, 
$44.3,42.7,40.5,39.4,38.3,36.4,34.7,33.7,32.7,31.7,30.7,30.6,30.0,29.7,28.7$, 22.0, 18.9, 11.5; HRMS (ESI) calculated for $\mathrm{C}_{45} \mathrm{H}_{50} \mathrm{NO}_{5}[\mathrm{M}+\mathrm{H}]^{+} 684.3644$, found 684.3691; purity 99.95\% $\left(\mathrm{CH}_{3} \mathrm{CN} / \mathrm{H}_{2} \mathrm{O}=90 / 10 ; \mathrm{R}_{\mathrm{t}}=17.729 \mathrm{~min}\right)$.

(6bS,8aS, 11R, 12bS, 14aR)-4,6b,8a,11,12b,14a-hexamethyl-2-oxo-11-((prop-2yn-1-yloxy)carbonyl)-2,6b, 7,8,8a,9,10,11,12,12a,12b,13,14,14a-

tetradecahydropicen-3-yl 10H-phenothiazine-10-carboxylate (4d): Yellow solid; yield $48 \%$; mp $196-197{ }^{\circ} \mathrm{C}$; $[\alpha]_{\mathrm{D}}^{24}+73.7\left(c=0.5\right.$ in $\left.\mathrm{CHCl}_{3}\right) ;{ }^{1} \mathrm{H}$ NMR $(600 \mathrm{MHz}$, $\left.\mathrm{CDCl}_{3}\right), \delta_{\mathrm{H}} 7.95(2 \mathrm{H}, \mathrm{s}, \mathrm{Ar}-\mathrm{H}), 7.04-7.38(6 \mathrm{H}, \mathrm{m}, \mathrm{Ar}-\mathrm{H}), 7.02(1 \mathrm{H}, \mathrm{d}, J=7.1 \mathrm{~Hz}, \mathrm{H}-6)$, $6.54(1 \mathrm{H}, \mathrm{s}, \mathrm{H}-1), 6.30(1 \mathrm{H}, \mathrm{d}, J=7.1 \mathrm{~Hz}, \mathrm{H}-7), 4.51-4.67(2 \mathrm{H}, \mathrm{m}, \mathrm{H}-1$ '), 2.43 (1H, s, $\left.\mathrm{C} \equiv \underline{\mathrm{CH}}, \mathrm{H}-3^{\prime}\right), 2.08(3 \mathrm{H}, \mathrm{s}, \mathrm{H}-23), 1.47(3 \mathrm{H}, \mathrm{s}, \mathrm{H}-25), 1.26(3 \mathrm{H}, \mathrm{s}, \mathrm{H}-26), 1.21(3 \mathrm{H}, \mathrm{s}$, H-30), 1.10 (3H, s, H-28), 0.58 (3H, s, H-28); ${ }^{13} \mathrm{C}$ NMR (150 MHz, CDCl $), \delta_{\mathrm{C}} 178.1$, $177.4,172.1,163.5,151.4,143.2,138.6,135.8,135.8(2 \times C), 132.0(2 \times C), 127.4(2$ $\times \mathrm{C}), 127.3(2 \times \mathrm{C}), 127.1(2 \times \mathrm{C}), 126.5,126.5(2 \times \mathrm{C}), 123.0,118.0,77.6,75.2,52.1$, $45.4,44.3,42.8,40.5,39.4,38.4,36.4,34.7,33.6,32.7,31.7,30.7,30.6,29.9,29.7$, 28.7, 22.0, 18.9, 11.5; HRMS (ESI) calculated for $\mathrm{C}_{45} \mathrm{H}_{48} \mathrm{NO}_{5} \mathrm{~S}[\mathrm{M}+\mathrm{H}]^{+}$714.3208, found 714.3254; purity $99.61 \%\left(\mathrm{CH}_{3} \mathrm{CN} / \mathrm{H}_{2} \mathrm{O}=90 / 10 ; \mathrm{R}_{\mathrm{t}}=25.983 \mathrm{~min}\right)$.

Prop-2-yn-1-yl $\quad(2 R, 4 a S, 6 a S, 12 b R, 14 a S)-10-(($ dimethylcarbamoyl)oxy)2,4a,6a,9,12b,14a-hexamethyl-11-oxo-1,2,3,4,4a,5,6,6a,11,12b,13,14,14a,14btetradecahydropicene-2-carboxylate (4e): Yellow solid; yield 61\%; mp 120-121 ${ }^{\circ} \mathrm{C}$; $[\alpha]_{\mathrm{D}}^{24}+38.5\left(c=0.5\right.$ in $\left.\mathrm{CHCl}_{3}\right) ;{ }^{1} \mathrm{H} \mathrm{NMR}\left(600 \mathrm{MHz}, \mathrm{CDCl}_{3}\right), \delta_{\mathrm{H}} 7.01(1 \mathrm{H}, \mathrm{d}, J=7.1 \mathrm{~Hz}$, H-6), 6.45 (1H, s, H-1), 6.30 (1H, d, J= 7.1 Hz, H-7), 4.49-4.66 (2H, m, H-1'), 2.43 $\left(1 \mathrm{H}, \mathrm{s}, \mathrm{C} \equiv \underline{\mathrm{CH}}, \mathrm{H}-3^{\prime}\right), 3.12\left(3 \mathrm{H}, \mathrm{s}, \mathrm{N}-\mathrm{CH}_{3}\right), 3.00\left(3 \mathrm{H}, \mathrm{s}, \mathrm{N}-\mathrm{CH}_{3}\right), 2.17(3 \mathrm{H}, \mathrm{s}, \mathrm{H}-23)$, 
$1.45(3 \mathrm{H}, \mathrm{s}, \mathrm{H}-25), 1.25$ (3H, s, H-26), 1.20 (3H, s, H-30), 1.09 (3H, s, H-28), 0.57 (3H, s, H-27); ${ }^{13} \mathrm{C}$ NMR (150 MHz, $\left.\mathrm{CDCl}_{3}\right), \delta_{\mathrm{C}} 178.7,177.4,171.1,163.0,154.3,143.3$, $134.9,133.4,126.8,123.1,117.9,77.5,75.2,52.0,45.2,44.3,42.6,40.5,39.4,38.3$, $36.9,36.8,36.4,34.7,33.7,32.7,31.7,30.7,30.6,30.0,29.7,28.7,22.0,18.9,11.3$; HRMS (ESI) calculated for $\mathrm{C}_{35} \mathrm{H}_{46} \mathrm{NO}_{5}[\mathrm{M}+\mathrm{H}]^{+}$560.3331, found 560.3379; purity $99.20 \%\left(\mathrm{CH}_{3} \mathrm{CN} / \mathrm{H}_{2} \mathrm{O}=90 / 10 ; \mathrm{R}_{\mathrm{t}}=10.611 \mathrm{~min}\right)$.

$(6 b S, 8 a S, 11 R, 12 b S, 14 a R)-4,6 b, 8 a, 11,12 b, 14 a$-hexamethyl-2-oxo-11-((prop-2-yn1-yloxy)carbonyl)-2,6b, 7,8,8a,9,10,11,12,12a,12b,13,14,14a-tetradecahydropicen-3yl [1,4'-bipiperidine]-1'-carboxylate (4f): Yellow solid; yield 38\%; mp 139-140 ${ }^{\circ} \mathrm{C} ;[\alpha]$ ${ }_{\mathrm{D}}^{24}+35.0\left(c=0.5\right.$ in $\left.\mathrm{CHCl}_{3}\right) ;{ }^{1} \mathrm{H}$ NMR $\left(600 \mathrm{MHz}, \mathrm{CDCl}_{3}\right), \delta_{\mathrm{H}} 6.97(1 \mathrm{H}, \mathrm{d}, J=7.1 \mathrm{~Hz}$, H-6), 6.39 (1H, s, H-1), 6.25 (1H, d, $J=7.1 \mathrm{~Hz}, \mathrm{H}-7), 4.42-4.62$ (2H, m, H-1'), 2.43 $\left(1 \mathrm{H}, \mathrm{s}, \mathrm{C} \equiv \underline{\mathrm{CH}}, \mathrm{H}-3^{\prime}\right), 2.75-2.98(3 \mathrm{H}, \mathrm{m}), 2.43-2.57(4 \mathrm{H}, \mathrm{m}), 2.12$ (3H, s, H-23), 1.40 (3H, s, H-25), 1.20 (3H, s, H-26), 1.15 (3H, s, H-30), 1.04 (3H, s, H-28), 0.52 (3H, s, $\mathrm{H}-27) ;{ }^{13} \mathrm{C} \mathrm{NMR}\left(150 \mathrm{MHz}, \mathrm{CDCl}_{3}\right), \delta_{\mathrm{C}} 178.4,177.2,170.9,162.9,153.0,143.2,134.9$, $133.2,126.7,122.9,117.8,77.5,75.1,62.6,51.9,50.1(2 \times C), 45.1,44.9(2 \times C), 44.3$, $44.2,42.5,40.3,39.3,38.2,36.3,34.6,33.6,32.5,30.6,30.5,29.8,29.5,28.6,27.9(2$ $\times$ C), $26.3(2 \times \mathrm{C}), 24.7,21.9,18.7,11.2 ; \mathrm{HRMS}(\mathrm{ESI})$ calculated for $\mathrm{C}_{43} \mathrm{H}_{59} \mathrm{~N}_{2} \mathrm{O}_{5}[\mathrm{M}$ $+\mathrm{H}]^{+}$683.4379, found 683.4426; purity $99.47 \%\left(\mathrm{CH}_{3} \mathrm{OH} / \mathrm{H}_{2} \mathrm{O}=90 / 10 ; \mathrm{R}_{t}=11.193\right.$ $\min )$.

(6bS,8aS, 11R, 12bS,14aR)-4,6b,8a,11,12b,14a-hexamethyl-2-oxo-11-((prop-2yn-1-yloxy)carbonyl)-2, 6b, 7,8,8a,9,10,11,12,12a,12b,13,14,14atetradecahydropicen-3-yl 10,11-dihydro-5H-dibenzo[b,f]azepine-5-carboxylate (4g): 
Yellow solid; yield $62 \%$; mp $184-185^{\circ} \mathrm{C} ;[\alpha]_{\mathrm{D}}^{24}+54.5\left(c=0.5\right.$ in $\left.\mathrm{CHCl}_{3}\right) ;{ }^{1} \mathrm{H} \mathrm{NMR}(600$ $\left.\mathrm{MHz}, \mathrm{CDCl}_{3}\right), \delta_{\mathrm{H}} 7.46-7.89(2 \mathrm{H}, \mathrm{m}, \mathrm{Ar}-\mathrm{H}), 7.16-7.22(6 \mathrm{H}, \mathrm{m}, \mathrm{Ar}-\mathrm{H}), 6.98(1 \mathrm{H}, \mathrm{d}, J$ =7.1 Hz, H-6), $6.46(1 \mathrm{H}, \mathrm{s}, \mathrm{H}-1), 6.27(1 \mathrm{H}, \mathrm{d}, J=7.1 \mathrm{~Hz}, \mathrm{H}-7), 4.47-4.67$ (2H, m, H-

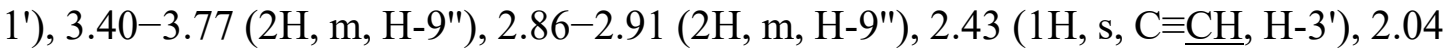
(3H, s, H-23), 1.42 (3H, s, H-25), 1.24 (3H, s, H-26), 1.20 (3H, s, H-30), 1.09 (3H, s, $\mathrm{H}-28), 0.56(3 \mathrm{H}, \mathrm{s}, \mathrm{H}-27) ;{ }^{13} \mathrm{C} \mathrm{NMR}\left(150 \mathrm{MHz}, \mathrm{CDCl}_{3}\right), \delta_{\mathrm{C}} 178.0,177.3,171.1,163.1$, 152.7, 143.3, $141.6(2 \times \mathrm{C}), 140.1(2 \times \mathrm{C}), 135.0,133.1,130.3(2 \times \mathrm{C}), 128.6(2 \times \mathrm{C})$, $127.8(2 \times \mathrm{C}), 127.5(2 \times \mathrm{C}), 126.6,122.9,117.9,77.5,75.1,52.0,45.2,44.3,42.6$, $40.4,39.3,38.2,36.4,34.7,33.6,32.6,31.6,31.2(2 \times \mathrm{C}), 30.6,30.6,29.9,29.6,28.7$, 22.0, 18.8, 11.4; HRMS (ESI) calculated for $\mathrm{C}_{47} \mathrm{H}_{52} \mathrm{NO}_{5}[\mathrm{M}+\mathrm{H}]^{+}$710.3801, found 710.3847; purity 99.78\% $\left(\mathrm{CH}_{3} \mathrm{CN} / \mathrm{H}_{2} \mathrm{O}=90 / 10 ; \mathrm{R}_{\mathrm{t}}=18.368 \mathrm{~min}\right)$.

(6bS,8aS, 11R, 12bS,14aR)-4,6b,8a,11,12b,14a-hexamethyl-2-oxo-11-((prop-2yn-1-yloxy)carbonyl)-2, 6b, 7,8,8a,9,10,11,12,12a,12b,13,14,14atetradecahydropicen-3-yl piperidine-1-carboxylate (4h): Yellow solid; yield 54\%; mp

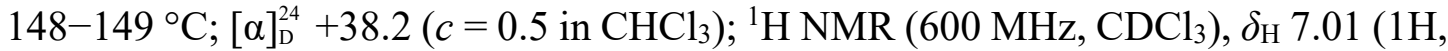
d, $J=7.1 \mathrm{~Hz}, \mathrm{H}-6), 6.43(1 \mathrm{H}, \mathrm{s}, \mathrm{H}-1), 6.29(1 \mathrm{H}, \mathrm{d}, J=7.1 \mathrm{~Hz}, \mathrm{H}-7), 4.46-4.67(2 \mathrm{H}, \mathrm{m}$, H-1'), $2.43\left(1 \mathrm{H}, \mathrm{s}, \mathrm{C} \equiv \underline{\mathrm{CH}}, \mathrm{H}-3^{\prime}\right), 2.16(3 \mathrm{H}, \mathrm{s}, \mathrm{H}-23), 1.44$ (3H, s, H-25), 1.25 (3H, s, H26), 1.19 (3H, s, H-30), 1.09 (3H, s, H-28), 0.57 (3H, s, H-27); ${ }^{13} \mathrm{C}$ NMR (150 MHz, $\left.\mathrm{CDCl}_{3}\right), \delta_{\mathrm{C}} 178.7,177.4,170.9,162.9,153.2,143.4,134.8,133.2,126.9,123.1,117.9$, $77.5,75.2,52.0,46.2,45.5,45.2,44.3,42.6,40.5,39.4,38.3,36.5,34.7,33.7,32.7$, $31.7,30.7,30.6,30.0,29.7,28.7,25.9,25.5,24.5,22.0,18.9,11.3$; HRMS (ESI) calculated for $\mathrm{C}_{38} \mathrm{H}_{50} \mathrm{NO}_{5}[\mathrm{M}+\mathrm{H}]^{+}$600.3644, found 600.3691 ; purity $99.81 \%$ 
$\left(\mathrm{CH}_{3} \mathrm{CN} / \mathrm{H}_{2} \mathrm{O}=90 / 10 ; \mathrm{R}_{\mathrm{t}}=14.671 \mathrm{~min}\right)$.

(6bS,8aS, 11R, 12bS,14aR)-4,6b,8a,11,12b,14a-hexamethyl-2-oxo-11-((prop-2yn-1-yloxy)carbonyl)-2,6b, 7,8,8a,9,10,11,12,12a,12b,13,14,14a-

tetradecahydropicen-3-yl pyrrolidine-1-carboxylate (4i): Yellow solid; yield 42\%; mp $143-144{ }^{\circ} \mathrm{C} ;[\alpha]_{\mathrm{D}}^{24}+27.3\left(c=0.5\right.$ in $\left.\mathrm{CHCl}_{3}\right) ;{ }^{1} \mathrm{H}$ NMR $\left(600 \mathrm{MHz}, \mathrm{CDCl}_{3}\right), \delta_{\mathrm{H}} 7.00$ (1H, d, $J=7.1 \mathrm{~Hz}, \mathrm{H}-6), 6.44(1 \mathrm{H}, \mathrm{s}, \mathrm{H}-1), 6.29(1 \mathrm{H}, \mathrm{d}, J=7.1 \mathrm{~Hz}, \mathrm{H}-7), 4.48-4.65$ (2H, m, H-1'), $2.43(1 \mathrm{H}, \mathrm{s}, \mathrm{C} \equiv \underline{\mathrm{CH}}, \mathrm{H}-3$ '), 2.18 (3H, s, H-23), 1.44 (3H, s, H-25), 1.25 (3H, s, H-26), 1.19 (3H, s, H-30), 1.08 (3H, s, H-28), 0.57 (3H, s, H-27); ${ }^{13} \mathrm{C}$ NMR (150 $\left.\mathrm{MHz}, \mathrm{CDCl}_{3}\right), \delta_{\mathrm{C}} 178.8,177.4,170.9,162.9,152.6,143.3,134.8,133.4,126.9,123.2$, $117.9,77.5,75.2,52.0,46.6,46.6,45.2,44.3,42.6,40.5,39.4,38.3,36.4,34.7,33.7$, 32.7, 31.7, 30.7, 30.6, 30.0, 29.7, 28.7, 25.9, 25.1, 22.0, 18.9, 11.4; HRMS (ESI) calculated for $\mathrm{C}_{37} \mathrm{H}_{48} \mathrm{NO}_{5}[\mathrm{M}+\mathrm{H}]^{+}$586.3488, found 586.3536; purity $99.56 \%$ $\left(\mathrm{CH}_{3} \mathrm{CN} / \mathrm{H}_{2} \mathrm{O}=90 / 10 ; \mathrm{R}_{\mathrm{t}}=11.624 \mathrm{~min}\right)$.

(6bS,8aS, 11R, 12bS,14aR)-4,6b,8a,11,12b,14a-hexamethyl-2-oxo-11-((prop-2yn-1-yloxy)carbonyl)-2, 6b, 7,8,8a,9,10,11,12,12a,12b,13,14,14a-

tetradecahydropicen-3-yl 5H-dibenzo[b,f]azepine-5-carboxylate (4j): Yellow solid; yield $55 \%$; mp $187-188^{\circ} \mathrm{C} ;[\alpha]_{\mathrm{D}}^{24}+62.2\left(c=0.5\right.$ in $\left.\mathrm{CHCl}_{3}\right) ;{ }^{1} \mathrm{HNMR}\left(600 \mathrm{MHz}, \mathrm{CDCl}_{3}\right)$, $\delta_{\mathrm{H}} 7.96(1 \mathrm{H}, \mathrm{s}, \mathrm{Ar}-\mathrm{H}), 7.66(1 \mathrm{H}, \mathrm{s}, \mathrm{Ar}-\mathrm{H}), 7.25-7.41(6 \mathrm{H}, \mathrm{m}, \mathrm{Ar}-\mathrm{H}), 6.94-6.97(2 \mathrm{H}, \mathrm{m}$, Ar-H), $6.94(1 \mathrm{H}, \mathrm{d}, J=7.1 \mathrm{~Hz}, \mathrm{H}-6), 6.46(1 \mathrm{H}, \mathrm{s}, \mathrm{H}-1), 6.24(1 \mathrm{H}, \mathrm{d}, J=7.1 \mathrm{~Hz}, \mathrm{H}-7)$, 4.47-4.66 (2H, m, H-1'), $2.43\left(1 \mathrm{H}, \mathrm{s}, \mathrm{C} \equiv \underline{\mathrm{CH}}, \mathrm{H}-3^{\prime}\right), 2.03(3 \mathrm{H}, \mathrm{s}, \mathrm{H}-23), 1.39$ (3H, s, H25), 1.22 (3H, s, H-26), 1.20 (3H, s, H-30), 1.08 (3H, s, H-28), 0.55 (3H, s, H-27); ${ }^{13} \mathrm{C}$ NMR $\left(150 \mathrm{MHz}, \mathrm{CDCl}_{3}\right), \delta_{\mathrm{C}} 178.0,177.3,171.0,162.9,152.5,143.1,139.9(2 \times \mathrm{C})$, 
135.0, 134.1, $133.2(2 \times \mathrm{C}), 130.7(2 \times \mathrm{C}), 130.2(2 \times \mathrm{C}), 129.1(2 \times \mathrm{C}), 128.8(2 \times \mathrm{C})$, $127.4(2 \times \mathrm{C}), 126.6,123.0,117.8,77.5,75.1,52.0,45.2,44.2,42.5,40.4,39.3,38.2$, $36.3,34.6,33.5,32.6,31.6,30.6,30.5,29.9,29.5,28.6,21.9,18.8,11.2$; HRMS (ESI) calculated for $\mathrm{C}_{47} \mathrm{H}_{50} \mathrm{NO}_{5}[\mathrm{M}+\mathrm{H}]^{+}$708.3644, found 708.3691 ; purity $99.43 \%$ $\left(\mathrm{CH}_{3} \mathrm{CN} / \mathrm{H}_{2} \mathrm{O}=90 / 10 ; \mathrm{R}_{\mathrm{t}}=17.320 \mathrm{~min}\right)$.

$(6 b S, 8 a S, 11 R, 12 b S, 14 a R)-4,6 b, 8 a, 11,12 b, 14 a$-hexamethyl-2-oxo-11-((prop-2yn-1-yloxy)carbonyl)-2,6b, 7,8,8a,9,10,11,12,12a,12b,13,14,14a-tetradecahydropicen3-yl 4-methylpiperazine-1-carboxylate (4k): Yellow solid; yield 41\%; mp 154-156 ${ }^{\circ} \mathrm{C}$; $[\alpha]_{\mathrm{D}}^{24}+61.2\left(c=0.5\right.$ in $\left.\mathrm{CHCl}_{3}\right) ;{ }^{1} \mathrm{H} \mathrm{NMR}\left(600 \mathrm{MHz}, \mathrm{CDCl}_{3}\right), \delta_{\mathrm{H}} 7.00(1 \mathrm{H}, \mathrm{d}, J=7.1 \mathrm{~Hz}$, H-6), 6.40 (1H, s, H-1), 6.27 (1H, d, $J=7.1 \mathrm{~Hz}, \mathrm{H}-7), 4.43-4.63$ (2H, m, H-1'), 3.54-4.68 (4H, m, H-3"), $2.43(1 \mathrm{H}, \mathrm{s}, \mathrm{C} \equiv \underline{\mathrm{CH}}, \mathrm{H}-3$ '), 2.41-2.44 (4H, m), $2.43(1 \mathrm{H}, \mathrm{s}$, $\left.\mathrm{C} \equiv \underline{\mathrm{CH}}, \mathrm{H}-3^{\prime}\right), 2.30\left(3 \mathrm{H}, \mathrm{s}, \mathrm{N}-\mathrm{CH}_{3}\right), 2.14(3 \mathrm{H}, \mathrm{s}, \mathrm{H}-23), 1.41(3 \mathrm{H}, \mathrm{s}, \mathrm{H}-25), 1.22(3 \mathrm{H}, \mathrm{s}$, H-26), 1.16 (3H, s, H-30), 1.05 (3H, s, H-28), 0.53 (3H, s, H-27); ${ }^{13} \mathrm{C}$ NMR (150 MHz, $\left.\mathrm{CDCl}_{3}\right), \delta_{\mathrm{C}} 178.4,177.3,171.2,163.1,153.0,143.2,135.1,133.3,126.6,122.9,117.8$, $77.4,75.1,54.7,54.5,51.9,46.2,45.2,44.9,44.2,44.0,42.6,40.4,39.3,38.3,36.3$, 34.6, 33.6, 32.6, 31.6, 30.6, 30.5, 29.9, 29.6, 28.6, 21.9, 18.8, 11.3; HRMS (ESI) calculated for $\mathrm{C}_{38} \mathrm{H}_{51} \mathrm{~N}_{2} \mathrm{O}_{5}[\mathrm{M}+\mathrm{H}]^{+}$615.3753, found 615.3801 ; purity $99.17 \%$ $\left(\mathrm{CH}_{3} \mathrm{OH} / \mathrm{H}_{2} \mathrm{O}=90 / 10 ; \mathrm{R}_{\mathrm{t}}=14.624 \mathrm{~min}\right)$.

Prop-2-yn-1-yl (2R,4aS, 6aS, 12bR,14aS)-10-((dipropylcarbamoyl)oxy)2,4a,6a,9,12b,14a-hexamethyl-11-oxo-1,2,3,4,4a,5,6,6a,11,12b,13,14,14a,14btetradecahydropicene-2-carboxylate (4l): Yellow solid; yield 52\%; mp 162-164 ${ }^{\circ} \mathrm{C}$; $[\alpha]_{\mathrm{D}}^{24}+43.3\left(c=0.5\right.$ in $\left.\mathrm{CHCl}_{3}\right) ;{ }^{1} \mathrm{H} \mathrm{NMR}\left(600 \mathrm{MHz}, \mathrm{CDCl}_{3}\right), \delta_{\mathrm{H}} 6.98(1 \mathrm{H}, \mathrm{d}, J=7.1 \mathrm{~Hz}$, 
H-6), 6.41 (1H, s, H-1), 6.27 (1H, d, $J=7.1 \mathrm{~Hz}, \mathrm{H}-7), 4.46-4.64$ (2H, m, H-1'), 3.23-3.33 (4H, m, H-3"), $2.43(1 \mathrm{H}, \mathrm{s}, \mathrm{C} \equiv \underline{\mathrm{CH}}, \mathrm{H}-3$ '), $2.15(3 \mathrm{H}, \mathrm{s}, \mathrm{H}-23), 1.42(3 \mathrm{H}, \mathrm{s}, \mathrm{H}-$ 25), 1.23 (3H, s, H-26), 1.18 (3H, s, H-30), 1.07 (3H, s, H-28), 0.55 (3H, s, H-27); ${ }^{13} \mathrm{C}$ NMR $\left(150 \mathrm{MHz}, \mathrm{CDCl}_{3}\right), \delta_{\mathrm{C}} 178.6,177.3,170.6,162.8,154.0,143.4,134.6,133.2$, $126.9,123.1,117.8,77.5,75.1,52.0,49.7,49.7,45.1,44.3,42.5,40.4,39.3,38.2,36.4$, $34.7,33.6,32.6,31.6,30.6,30.6,29.9,29.6,28.7,22.0,22.0,21.3,18.8,11.4,11.3$, 11.3; (+)-HRESIMS $m / z 616.3835[\mathrm{M}+\mathrm{H}]^{+}\left(\right.$calcd for $\left.\mathrm{C}_{39} \mathrm{H}_{54} \mathrm{NO}_{5}, 616.3957\right)$. HRMS (ESI) calculated for $\mathrm{C}_{39} \mathrm{H}_{54} \mathrm{NO}_{5}[\mathrm{M}+\mathrm{H}]^{+}$616.3957, found 616.3835 ; purity $98.46 \%$ $\left(\mathrm{CH}_{3} \mathrm{CN} / \mathrm{H}_{2} \mathrm{O}=90 / 10 ; \mathrm{R}_{\mathrm{t}}=19.768 \mathrm{~min}\right)$.

Prop-2-yn-1-yl $\quad(2 R, 4 a S, 6 a S, 12 b R, 14 a S)-10-(($ ethyl(methyl)carbamoyl)oxy)2,4a,6a,9,12b,14a-hexamethyl-11-oxo-1,2,3,4,4a,5,6,6a,11,12b,13,14,14a,14btetradecahydropicene-2-carboxylate (4m): Yellow solid; yield 57\%; mp 139-140 ${ }^{\circ} \mathrm{C}$; $[\alpha]_{\mathrm{D}}^{24}+35.9\left(c=0.5\right.$ in $\left.\mathrm{CHCl}_{3}\right) ;{ }^{1} \mathrm{H} \mathrm{NMR}\left(600 \mathrm{MHz}, \mathrm{CDCl}_{3}\right), \delta_{\mathrm{H}} 6.99(1 \mathrm{H}, \mathrm{d}, J=7.1 \mathrm{~Hz}$, H-6), $6.41(1 \mathrm{H}, \mathrm{s}, \mathrm{H}-1), 6.28(1 \mathrm{H}, \mathrm{d}, J=7.1 \mathrm{~Hz}, \mathrm{H}-7), 4.46-4.64$ (2H, m, H-1'), 3.26-3.37 (2H, m, H-3"), 2.43 (1H, s, CE $\underline{\mathrm{CH}}, \mathrm{H}-3$ '), 2.15 (3H, s, H-23), 1.42 (3H, s, H25), 1.23 (3H, s, H-26), 1.18 (3H, s, H-30), 1.07 (3H, s, H-28), 0.55 (3H, s, H-27); ${ }^{13} \mathrm{C}$ NMR (150 MHz, $\left.\mathrm{CDCl}_{3}\right), \delta_{\mathrm{C}} 178.7,177.4,170.9,162.9,154.1,143.4,134.9,133.3$, $126.9,123.1,117.9,77.5,75.2,52.0,51.4,45.2,44.3,42.6,40.4,39.3,38.3,36.4,35.0$, 34.7, 33.6, 32.6, 31.7, 30.7, 30.6, 30.0, 29.7, 28.7, 22.0, 21.2, 18.8, 11.3, 11.1;HRMS (ESI) calculated for $\mathrm{C}_{37} \mathrm{H}_{50} \mathrm{NO}_{5}[\mathrm{M}+\mathrm{H}]^{+}$588.3644, found 588.3678; purity $99.82 \%$ $\left(\mathrm{CH}_{3} \mathrm{CN} / \mathrm{H}_{2} \mathrm{O}=90 / 10 ; \mathrm{R}_{\mathrm{t}}=13.712 \mathrm{~min}\right)$.

Prop-2-yn-1-yl (2R,4aS,6aS,12bR,14aS)-10-((ethyl(methyl)carbamoyl)oxy)- 
2,4a,6a,9,12b,14a-hexamethyl-11-oxo-1,2,3,4,4a,5,6,6a,11,12b,13,14,14a,14btetradecahydropicene-2-carboxylate (4n): Yellow solid; yield 54\%; mp $163-164{ }^{\circ} \mathrm{C}$; $[\alpha]_{\mathrm{D}}^{24}+44.3\left(c=0.5\right.$ in $\left.\mathrm{CHCl}_{3}\right) ;{ }^{1} \mathrm{H} \mathrm{NMR}\left(600 \mathrm{MHz}, \mathrm{CDCl}_{3}\right), \delta_{\mathrm{H}} 7.00(1 \mathrm{H}, \mathrm{d}, J=7.1 \mathrm{~Hz}$, H-6), $6.43(1 \mathrm{H}, \mathrm{s}, \mathrm{H}-1), 6.29(1 \mathrm{H}, \mathrm{d}, J=7.1 \mathrm{~Hz}, \mathrm{H}-7), 4.47-4.65(2 \mathrm{H}, \mathrm{m}, \mathrm{H}-1$ '), 3.31-3.42 (2H, m, H-3"), 2.43 (1H, s, C $\equiv \underline{\mathrm{CH}}, \mathrm{H}-3$ '), 2.16 (3H, s, H-23), 1.44 (3H, s, H25), 1.25 (3H, s, H-26), 1.19 (3H, s, H-30), 1.08 (3H, s, H-28), $0.56(3 \mathrm{H}, \mathrm{s}, \mathrm{H}-27) ;{ }^{13} \mathrm{C}$ NMR (150 MHz, $\left.\mathrm{CDCl}_{3}\right), \delta_{\mathrm{C}} 178.7,177.4,170.9,162.9,154.1,143.4,134.8,133.3$, $126.9,123.1,117.9,77.5,75.2,52.0,49.6,45.2,44.3,42.6,40.5,39.4,38.3,36.4,35.0$, $34.7,34.7,33.7,32.7,31.7,30.7,30.6,30.2,30.0,29.7,28.7,22.0,20.0,18.8,14.0$, 11.3; HRMS (ESI) calculated for $\mathrm{C}_{38} \mathrm{H}_{52} \mathrm{NO}_{5}[\mathrm{M}+\mathrm{H}]^{+}$602.3801, found 602.3834; purity $99.78 \%\left(\mathrm{CH}_{3} \mathrm{CN} / \mathrm{H}_{2} \mathrm{O}=90 / 10 ; \mathrm{R}_{\mathrm{t}}=16.296 \mathrm{~min}\right)$.

$$
\text { prop-2-yn-1-yl (2R,4aS,6aS, 12bR,14aS)-10-((dibutylcarbamoyl)oxy)- }
$$

2,4a,6a,9,12b,14a-hexamethyl-11-oxo-1,2,3,4,4a,5,6,6a,11,12b,13,14,14a,14b-

tetradecahydropicene-2-carboxylate (4o): Yellow solid; yield $64 \%$; mp $171-172{ }^{\circ} \mathrm{C}$; $[\alpha]_{\mathrm{D}}^{24}+49.8\left(c=0.5\right.$ in $\left.\mathrm{CHCl}_{3}\right) ;{ }^{1} \mathrm{H} \mathrm{NMR}\left(600 \mathrm{MHz}, \mathrm{CDCl}_{3}\right), \delta_{\mathrm{H}} 6.98(1 \mathrm{H}, \mathrm{d}, J=7.1 \mathrm{~Hz}$, H-6), $6.41(1 \mathrm{H}, \mathrm{s}, \mathrm{H}-1), 6.27(1 \mathrm{H}, \mathrm{d}, J=7.1 \mathrm{~Hz}, \mathrm{H}-7), 4.46-4.64\left(2 \mathrm{H}, \mathrm{m}, \mathrm{H}-1^{\prime}\right)$,

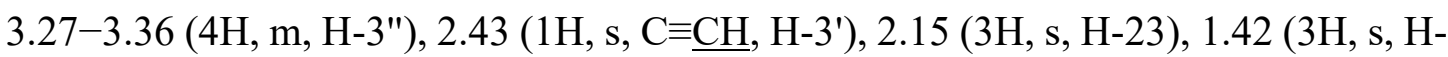
25), 1.23 (3H, s, H-26), 1.18 (3H, s, H-30), 1.07 (3H, s, H-28), 0.55 (3H, s, H-27); ${ }^{13} \mathrm{C}$ NMR $\left(150 \mathrm{MHz}, \mathrm{CDCl}_{3}\right), \delta_{\mathrm{C}} 178.6,177.3,170.7,162.8,154.0,143.4,134.6,133.2$, $126.9,123.1,117.8,77.5,75.2,52.0,47.7,47.7,45.2,44.3,42.5,40.4,39.3,38.2,36.4$, $34.7,33.6,32.6,31.6,30.9,30.6,30.6,30.1,29.9,29.6,28.7,22.0,20.2,20.0,18.8$, 14.0, 13.9, 11.3; HRMS (ESI) calculated for $\mathrm{C}_{41} \mathrm{H}_{58} \mathrm{NO}_{5}[\mathrm{M}+\mathrm{H}]^{+}$644.4270, found 
644.4303; purity 97.92\% $\left(\mathrm{CH}_{3} \mathrm{CN} / \mathrm{H}_{2} \mathrm{O}=90 / 10 ; \mathrm{R}_{\mathrm{t}}=11.388 \mathrm{~min}\right)$.

(6bS,8aS, 11R, 12bS,14aR)-4,6b,8a,11,12b,14a-hexamethyl-2-oxo-11-((prop-2yn-1-yloxy)carbonyl)-2,6b, 7,8,8a,9,10,11,12,12a,12b,13,14,14a-

tetradecahydropicen-3-yl 4-phenylpiperazine-1-carboxylate (4p): Yellow solid; yield $26 \%$; mp $143-145{ }^{\circ} \mathrm{C} ;[\alpha]_{\mathrm{D}}^{24}+36.3\left(c=0.5\right.$ in $\left.\mathrm{CHCl}_{3}\right) ;{ }^{1} \mathrm{H}$ NMR $\left(600 \mathrm{MHz}, \mathrm{CDCl}_{3}\right), \delta_{\mathrm{H}}$ 7.27-7.29 (2H, m, Ar-H), 7.04 (1H, d, J=7.1 Hz, H-6), 6.89-6.97 (3H, m, Ar-H), 6.46 (1H, s, H-1), $6.31(1 \mathrm{H}, \mathrm{d}, J=7.1 \mathrm{~Hz}, \mathrm{H}-7), 4.48-4.67(2 \mathrm{H}, \mathrm{m}, \mathrm{H}-1$ '), $3.73-3.86(4 \mathrm{H}, \mathrm{m}$, H-4"), 3.20-3.26 (4H, m, H-3"), 2.43 (1H, s, CE $\underline{\mathrm{CH}}, \mathrm{H}-3$ '), 2.19 (3H, s, H-23), 1.46 (3H, s, H-25), 1.26 (3H, s, H-26), 1.20 (3H, s, H-30), 1.10 (3H, s, H-28), 0.58 (3H, s, $\mathrm{H}-27) ;{ }^{13} \mathrm{C} \mathrm{NMR}\left(150 \mathrm{MHz}, \mathrm{CDCl}_{3}\right), \delta_{\mathrm{C}} 178.5,177.4,171.5,163.2,153.2,151.4,143.3$, 135.3, 133.5, $129.3(2 \times \mathrm{C}), 126.8,123.0,120.5,117.9,116.9(2 \times \mathrm{C}), 77.6,75.2,52.0$, $49.5(2 \times \mathrm{C}), 45.3,45.1,44.4,44.2,42.7,40.5,39.4,38.4,36.4,34.7,33.7,32.7,31.7$, 30.7, 30.6, 30.0, 29.7, 28.8, 22.0, 18.9, 11.4; HRMS (ESI) calculated for $\mathrm{C}_{43} \mathrm{H}_{53} \mathrm{~N}_{2} \mathrm{O}_{5}$ $[\mathrm{M}+\mathrm{H}]^{+}$677.3910, found 677.3942; purity $95.30 \%\left(\mathrm{CH}_{3} \mathrm{CN} / \mathrm{H}_{2} \mathrm{O}=90 / 10 ; \mathrm{R}_{\mathrm{t}}=15.439\right.$ $\min )$.

(6bS, 8aS, 11R, 12bS,14aR)-4,6b,8a,11,12b,14a-hexamethyl-2-oxo-11-((prop-2yn-1-yloxy)carbonyl)-2, 6b, 7,8,8a,9,10,11,12,12a,12b,13,14,14atetradecahydropicen-3-yl 4-(4-chlorophenyl)piperazine-1-carboxylate (4q): Yellow solid; yield 18\%; mp $146-148{ }^{\circ} \mathrm{C} ;[\alpha]_{\mathrm{D}}^{24}+64.0\left(c=0.5\right.$ in $\left.\mathrm{CHCl}_{3}\right) ;{ }^{1} \mathrm{H}$ NMR $(400 \mathrm{MHz}$, $\left.\mathrm{CDCl}_{3}\right), \delta_{\mathrm{H}} 7.23(2 \mathrm{H}, \mathrm{d}, J=8.0 \mathrm{~Hz}, \mathrm{Ar}-\mathrm{H}), 7.06(1 \mathrm{H}, \mathrm{d}, J=7.1 \mathrm{~Hz}, \mathrm{H}-6), 6.86(2 \mathrm{H}, \mathrm{d}$, $J=8.0 \mathrm{~Hz}, \mathrm{Ar}-\mathrm{H}), 6.45(1 \mathrm{H}, \mathrm{s}, \mathrm{H}-1), 6.33(1 \mathrm{H}, \mathrm{d}, J=7.1 \mathrm{~Hz}, \mathrm{H}-7), 4.48-4.67(2 \mathrm{H}, \mathrm{m}$, H-1'), 3.71-3.85 (4H, m, H-4"), 3.10-3.13 (4H, m, H-3"), 2.43 (1H, s, C三 $\left.\underline{\mathrm{CH}}, \mathrm{H}-3^{\prime}\right)$, 
$2.19(3 \mathrm{H}, \mathrm{s}, \mathrm{H}-23), 1.46$ (3H, s, H-25), $1.26(3 \mathrm{H}, \mathrm{s}, \mathrm{H}-26), 1.20(3 \mathrm{H}, \mathrm{s}, \mathrm{H}-30), 1.09(3 \mathrm{H}$, s, H-28), 0.58 (3H, s, H-27); ${ }^{13} \mathrm{C}$ NMR (100 MHz, $\left.\mathrm{CDCl}_{3}\right), \delta_{\mathrm{C}} 178.5,177.4,171.6$, $163.3,153.2,150.0,143.3,135.4,133.5,129.2(2 \times \mathrm{C}), 126.7,125.4,123.0,118.1(2 \times$ C), 118.0, 77.6, 75.2, 52.1, $49.5(2 \times \mathrm{C}), 45.3,45.1,44.4,44.1,42.8,40.5,39.5,38.4$, $36.5,34.8,33.7,32.7,31.7,30.7,30.7,30.0,29.7,28.8,22.0,18.9,11.4$; HRMS (ESI) calculated for $\mathrm{C}_{43} \mathrm{H}_{52} \mathrm{ClN}_{2} \mathrm{O}_{5}[\mathrm{M}+\mathrm{H}]^{+}$711.3520, found 711.3551 ; purity $97.01 \%$ $\left(\mathrm{CH}_{3} \mathrm{CN} / \mathrm{H}_{2} \mathrm{O}=90 / 10 ; \mathrm{R}_{\mathrm{t}}=18.594 \mathrm{~min}\right)$.

(6bS, 8aS, 11R, 12bS,14aR)-4,6b,8a,11,12b,14a-hexamethyl-2-oxo-11-((prop-2yn-1-yloxy)(arbonyl)-2,6b, 7,8,8a,9,10,11,12,12a,12b,13,14,14a-

tetradecahydropicen-3-yl 4-(2-chlorophenyl)piperazine-1-carboxylate (4r): Yellow solid; yield 61\%; mp $138-139{ }^{\circ} \mathrm{C} ;[\alpha]_{\mathrm{D}}^{24}+46.8\left(c=0.5\right.$ in $\left.\mathrm{CHCl}_{3}\right) ;{ }^{1} \mathrm{H}$ NMR $(400 \mathrm{MHz}$, $\left.\mathrm{CDCl}_{3}\right), \delta_{\mathrm{H}} 7.37(1 \mathrm{H}, \mathrm{d}, J=8.0 \mathrm{~Hz}, \mathrm{Ar}-\mathrm{H}), 7.20-7.25(1 \mathrm{H}, \mathrm{m}, \mathrm{Ar}-\mathrm{H}), 6.97-7.07(2 \mathrm{H}$, m, Ar-H), $7.04(1 \mathrm{H}, \mathrm{d}, J=7.1 \mathrm{~Hz}, \mathrm{H}-6), 6.45(1 \mathrm{H}, \mathrm{s}, \mathrm{H}-1), 6.31(1 \mathrm{H}, \mathrm{d}, J=7.1 \mathrm{~Hz}, \mathrm{H}-$ 7), 4.47-4.67 (2H, m, H-1'), 3.74-3.87 (4H, m, H-4"), 3.10-3.13 (4H, m, H-3"), 2.43 (1H, s, C $\left.\equiv \underline{\mathrm{CH}}, \mathrm{H}-3^{\prime}\right), 2.20$ (3H, s, H-23), 1.46 (3H, s, H-25), 1.26 (3H, s, H-26), 1.20 (3H, s, H-30), 1.09 (3H, s, H-28), 0.58 (3H, s, H-27); ${ }^{13} \mathrm{C} \mathrm{NMR}\left(100 \mathrm{MHz}, \mathrm{CDCl}_{3}\right), \delta_{\mathrm{C}}$ $178.5,177.4,171.4,163.2,153.3,149.1,143.3,135.2,133.4,130.7,129.1,127.8,126.8$, $124.2,123.0,120.8,117.9,77.5,75.2,52.0,51.2,51.1,45.5,45.3,44.6,44.3,42.7,40.5$, $39.4,38.4,36.4,34.7,33.7,32.7,31.7,30.7,30.6,29.9,29.6,28.7,22.0,18.9,11.4$; (+)-HRESIMS $m / z 711.3487[\mathrm{M}+\mathrm{H}]^{+}$(calcd for $\mathrm{C}_{43} \mathrm{H}_{52} \mathrm{ClN}_{2} \mathrm{O}_{5}, 711.3520$ ). HRMS (ESI) calculated for $\mathrm{C}_{43} \mathrm{H}_{52} \mathrm{ClN}_{2} \mathrm{O}_{5}[\mathrm{M}+\mathrm{H}]^{+} 711.3520$, found 711.3551 ; purity $99.50 \%$ $\left(\mathrm{CH}_{3} \mathrm{CN} / \mathrm{H}_{2} \mathrm{O}=90 / 10 ; \mathrm{R}_{\mathrm{t}}=20.920 \mathrm{~min}\right)$. 
$(6 b S, 8 a S, 11 R, 12 b S, 14 a R)-4,6 b, 8 a, 11,12 b, 14 a$-hexamethyl-2-oxo-11-((prop-2yn-1-yloxy)carbonyl)-2,6b, 7,8,8a,9,10,11,12,12a,12b,13,14,14a-

tetradecahydropicen-3-yl 4-(3-methoxyphenyl)piperazine-1-carboxylate $(4 s)$ : Yellow solid; yield 63\%; mp $131-133{ }^{\circ} \mathrm{C} ;[\alpha]_{\mathrm{D}}^{24}+51.7\left(c=0.5\right.$ in $\left.\mathrm{CHCl}_{3}\right) ;{ }^{1} \mathrm{H} \mathrm{NMR}(400$ $\left.\mathrm{MHz}, \mathrm{CDCl}_{3}\right), \delta_{\mathrm{H}} 7.16-7.20(1 \mathrm{H}, \mathrm{m}, \mathrm{Ar}-\mathrm{H}), 7.04(1 \mathrm{H}, \mathrm{d}, J=7.1 \mathrm{~Hz}, \mathrm{H}-6), \quad 6.44-6.57$ (4H, m, Ar-H), 6.30 (1H, d, J= 7.1 Hz, H-7), 4.47-4.67 (2H, m, H-1'), 3.71-3.84 (4H,

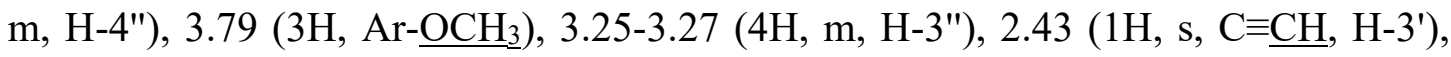
$2.19(3 \mathrm{H}, \mathrm{s}, \mathrm{H}-23), 1.46$ (3H, s, H-25), 1.26 (3H, s, H-26), 1.20 (3H, s, H-30), 1.09 (3H, s, H-28), 0.58 (3H, s, H-27); ${ }^{13} \mathrm{C}$ NMR (100 MHz, $\left.\mathrm{CDCl}_{3}\right), \delta_{\mathrm{C}} 178.4,177.3,171.4$, $163.1,160.6,153.1,152.6,143.2,135.1,133.4,129.9,126.6,122.9,117.8,109.4,105.2$ 103.1, 77.5, 75.1, 55.2, 51.9, $49.3(2 \times \mathrm{C}), 45.2,44.9,44.2,44.1,42.6,40.4,39.3,38.3$, 36.3, 34.6, 33.6, 32.6, 31.6, 30.6, 30.5, 29.9, 29.6, 28.6, 21.9, 18.8, 11.3; HRMS (ESI) calculated for $\mathrm{C}_{44} \mathrm{H}_{55} \mathrm{~N}_{2} \mathrm{O}_{6}[\mathrm{M}+\mathrm{H}]^{+} 707.4015$, found 707.4047 ; purity $98.45 \%$ $\left(\mathrm{CH}_{3} \mathrm{CN} / \mathrm{H}_{2} \mathrm{O}=90 / 10 ; \mathrm{R}_{\mathrm{t}}=15.365 \mathrm{~min}\right)$.

(6bS,8aS, 11R, 12bS,14aR)-4,6b,8a,11,12b,14a-hexamethyl-2-oxo-11-((prop-2yn-1-yloxy)carbonyl)-2, 6b, 7,8,8a,9,10,11,12,12a,12b,13,14,14atetradecahydropicen-3-yl 4-(2-methoxyphenyl)piperazine-1-carboxylate (4t): Yellow solid; yield 53\%; mp $127-129{ }^{\circ} \mathrm{C} ;[\alpha]_{\mathrm{D}}^{24}+35.7\left(c=0.5\right.$ in $\left.\mathrm{CHCl}_{3}\right) ;{ }^{1} \mathrm{H}$ NMR $(400$ $\left.\mathrm{MHz}, \mathrm{CDCl}_{3}\right), \delta_{\mathrm{H}} 6.99-7.04(2 \mathrm{H}, \mathrm{m}, \mathrm{Ar}-\mathrm{H}), 6.86-6.97$ (3H, m, Ar-H), 6.45 (1H, s, H1), 6.30 (1H, d, J= 7.1 Hz, H-7), 4.46-4.67 (2H, m, H-1'), 3.73-3.88 (4H, m, H-4"), $3.87\left(3 \mathrm{H}, \mathrm{Ar}-\underline{\mathrm{OCH}}_{3}\right), 3.10-3.12(4 \mathrm{H}, \mathrm{m}, \mathrm{H}-3 "), 2.43(1 \mathrm{H}, \mathrm{s}, \mathrm{C} \equiv \underline{\mathrm{CH}}, \mathrm{H}-3$ ') 2.19 (3H, s, H-23), 1.45 (3H, s, H-25), 1.26 (3H, s, H-26), 1.19 (3H, s, H-30), 1.08 (3H, s, H-28), 
$0.57(3 \mathrm{H}, \mathrm{s}, \mathrm{H}-27) ;{ }^{13} \mathrm{C} \mathrm{NMR}\left(100 \mathrm{MHz}, \mathrm{CDCl}_{3}\right), \delta_{\mathrm{C}} 178.5,177.3,171.2,163.1,153.1$, 152.4, 143.3, 141.1, 135.1, 133.4, 126.7, 123.4, 123.0, 121.1, 118.7, 117.9, 111.4, 77.5, $75.2,55.5,52.0,50.6(2 \times \mathrm{C}), 45.4,45.2,44.5,44.3,42.6,40.4,39.4,38.3,36.4,34.7$, 33.7, 32.6, 31.6, 30.7, 30.6, 29.9, 29.6, 28.7, 22.0, 18.8, 11.3; HRMS (ESI) calculated for $\mathrm{C}_{44} \mathrm{H}_{55} \mathrm{~N}_{2} \mathrm{O}_{6}[\mathrm{M}+\mathrm{H}]^{+}$707.4015, found 707.4046; purity 98.61\% $\left(\mathrm{CH}_{3} \mathrm{CN} / \mathrm{H}_{2} \mathrm{O}=\right.$ $\left.90 / 10 ; \mathrm{R}_{\mathrm{t}}=14.519 \mathrm{~min}\right)$.

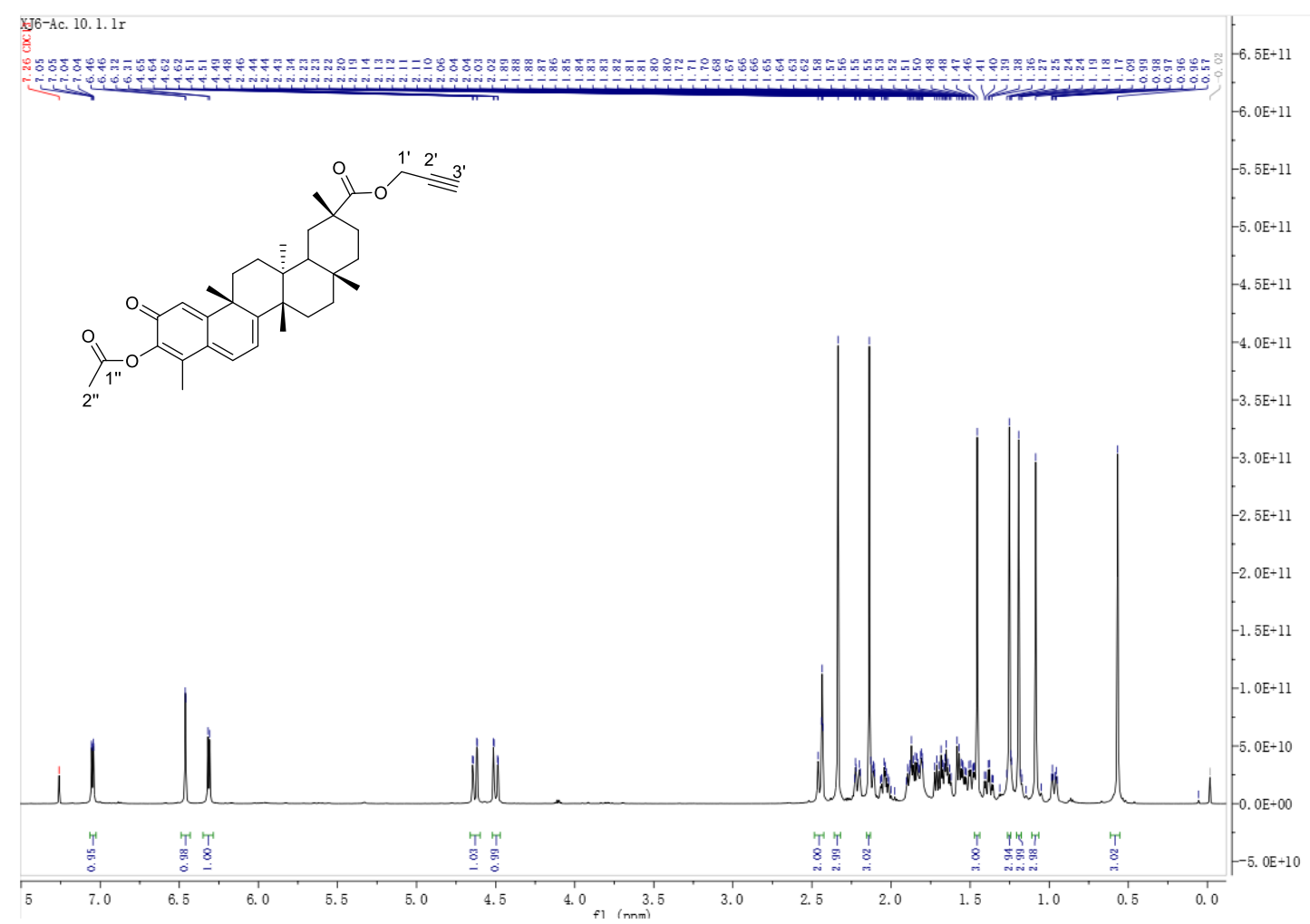

Figure S 1-1 ${ }^{1} \mathrm{H}$ NMR Spectrum $(600 \mathrm{MHz})$ of compound $\mathbf{2 a}$ in $\mathrm{CDCl}_{3}$ 


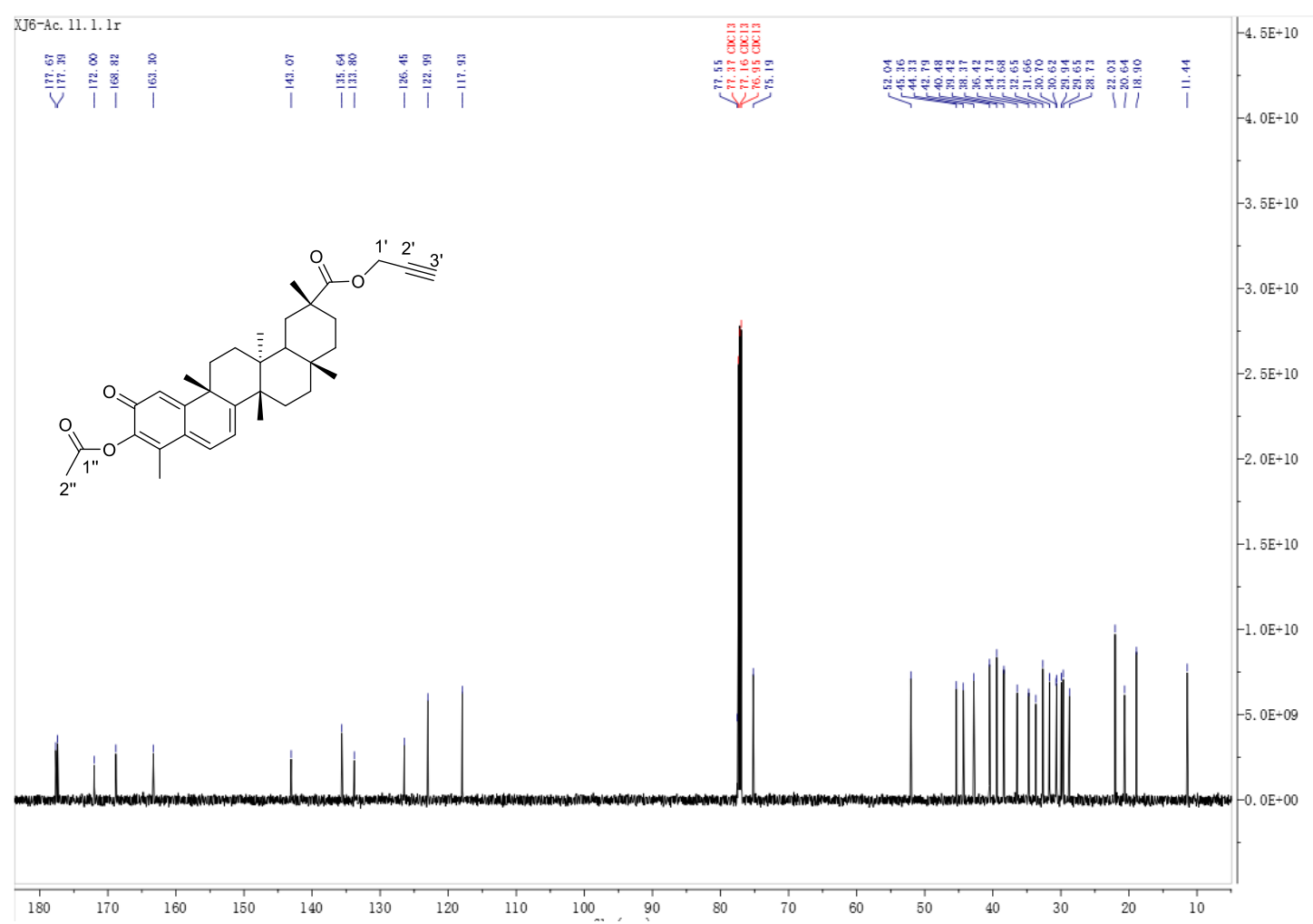

Figure S 1-2 ${ }^{13} \mathrm{C}$ NMR Spectrum (150MHz) of compound 2a in $\mathrm{CDCl}_{3}$

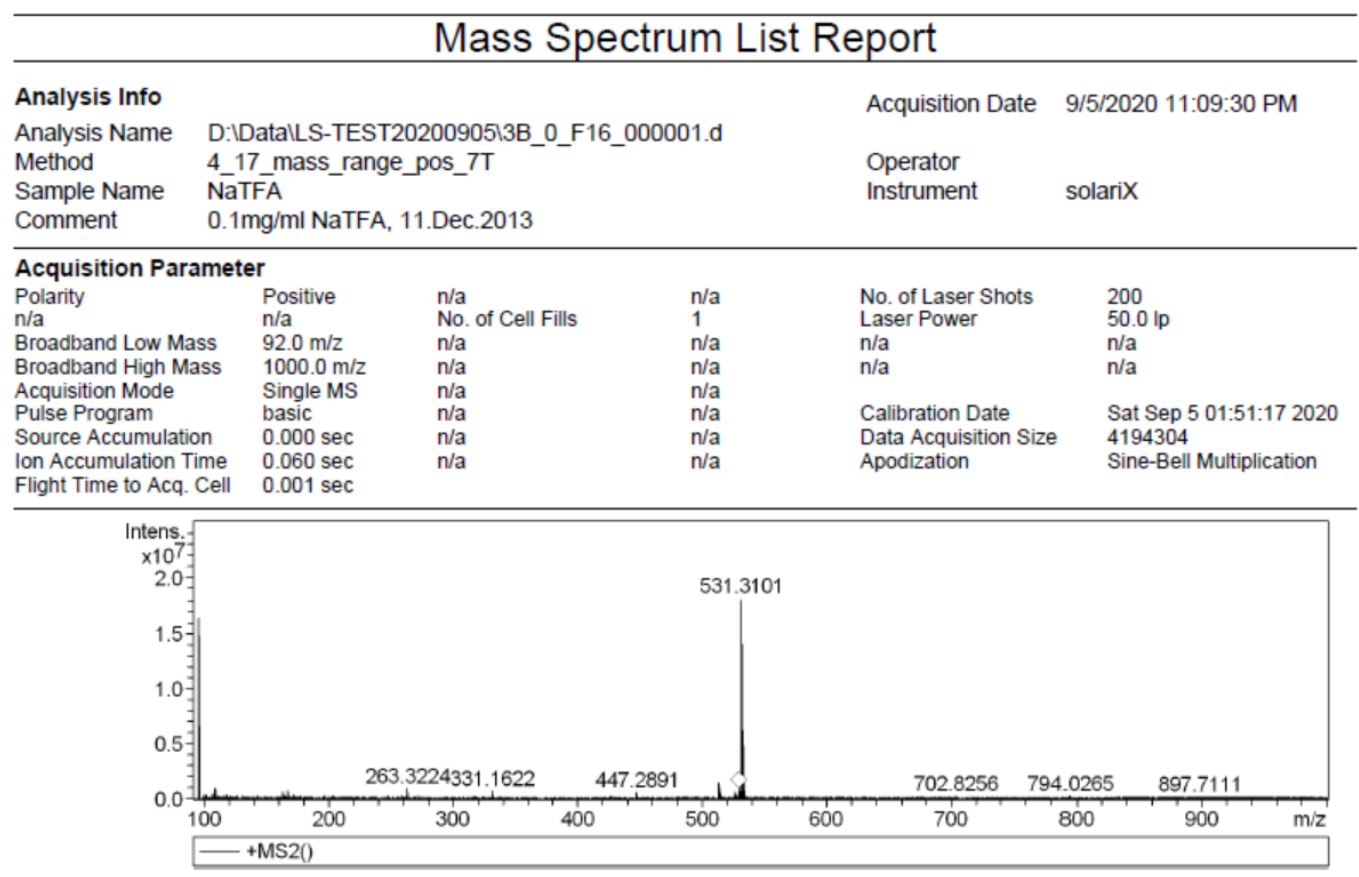

Figure S 1-4 HRESIMS Spectrum of compound 2a 
〈色谱图〉

$\mathrm{mV}$

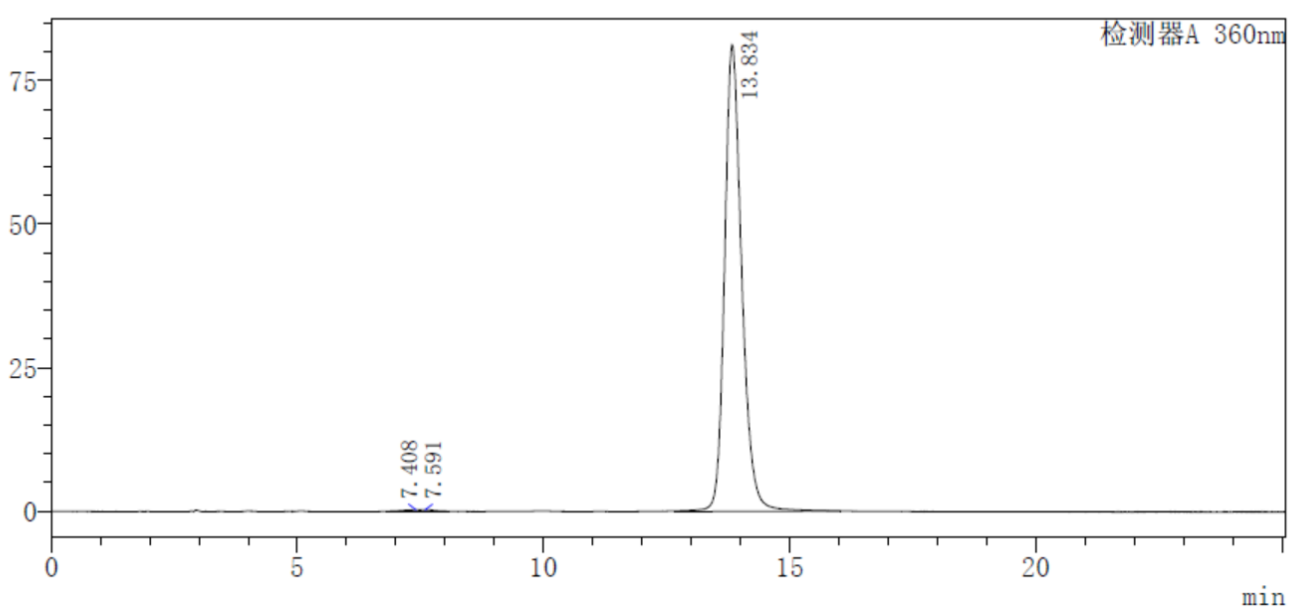

Figure S 1-3 HPLC Spectrum of compound 2a

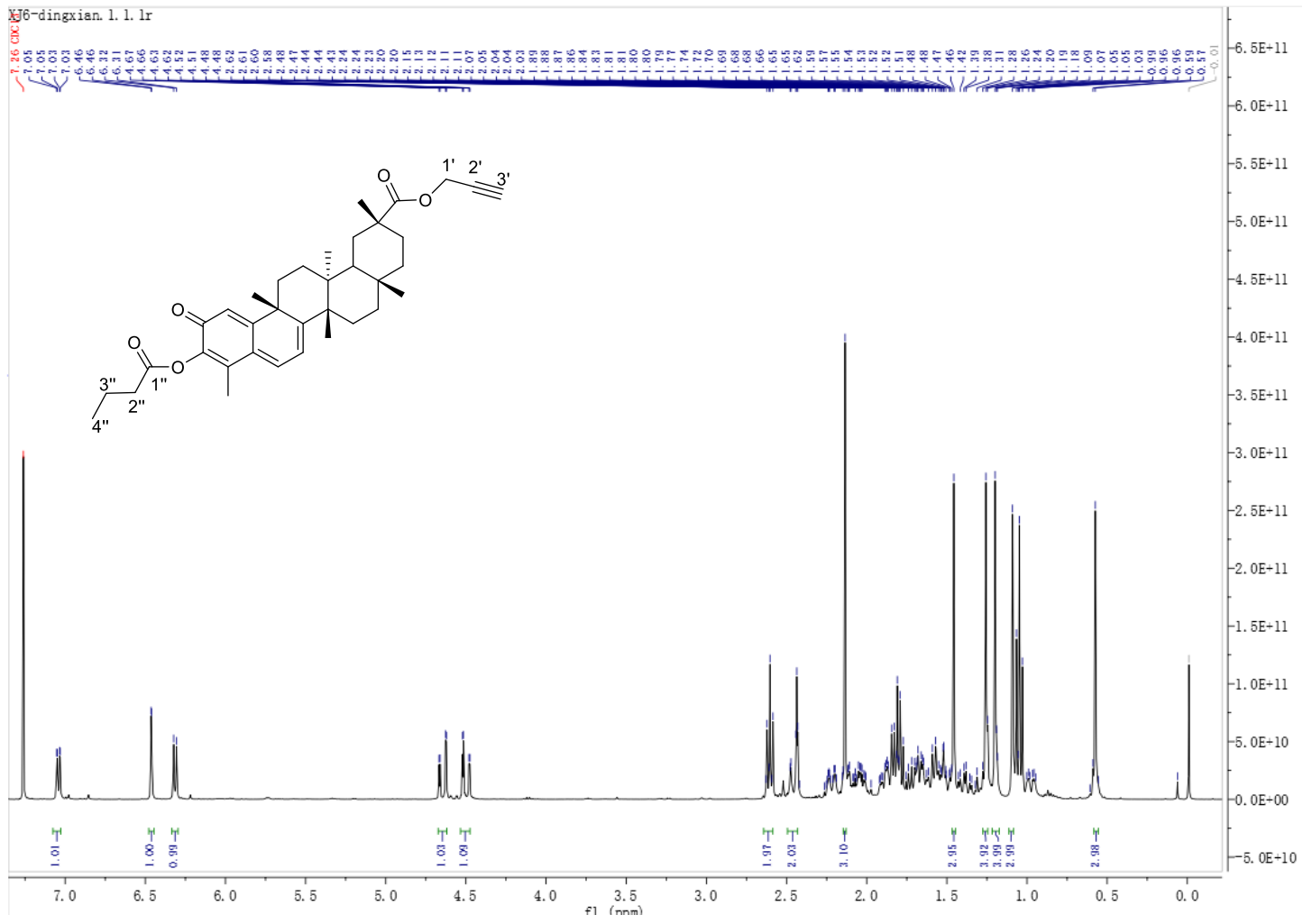

Figure S 2-1 ${ }^{1} \mathrm{H}$ NMR Spectrum $(600 \mathrm{MHz})$ of compound $\mathbf{2 b}$ in $\mathrm{CDCl}_{3}$ 


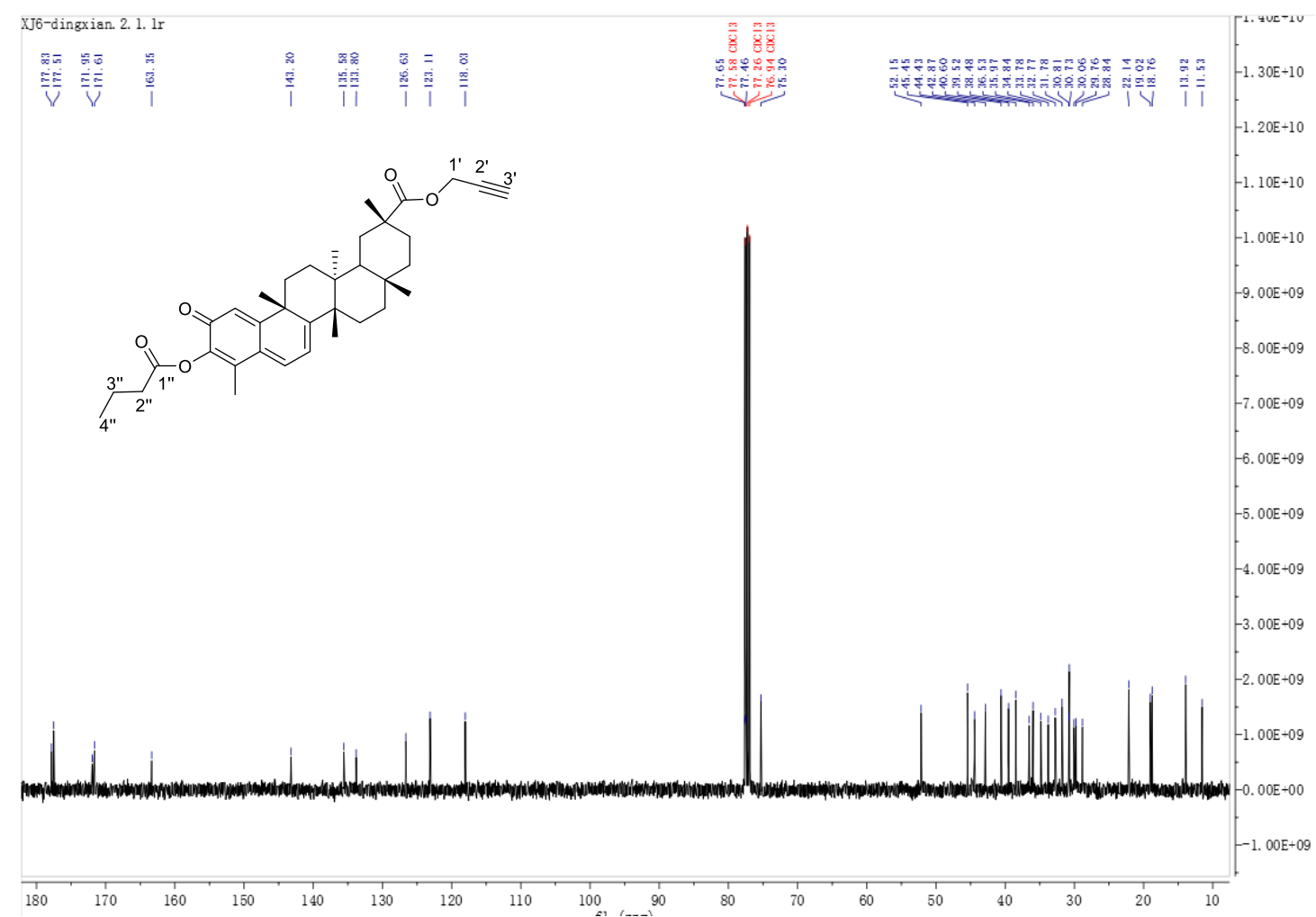

Figure S 2-2 ${ }^{13} \mathrm{C}$ NMR Spectrum $(150 \mathrm{MHz})$ of compound $\mathbf{2 b}$ in $\mathrm{CDCl}_{3}$

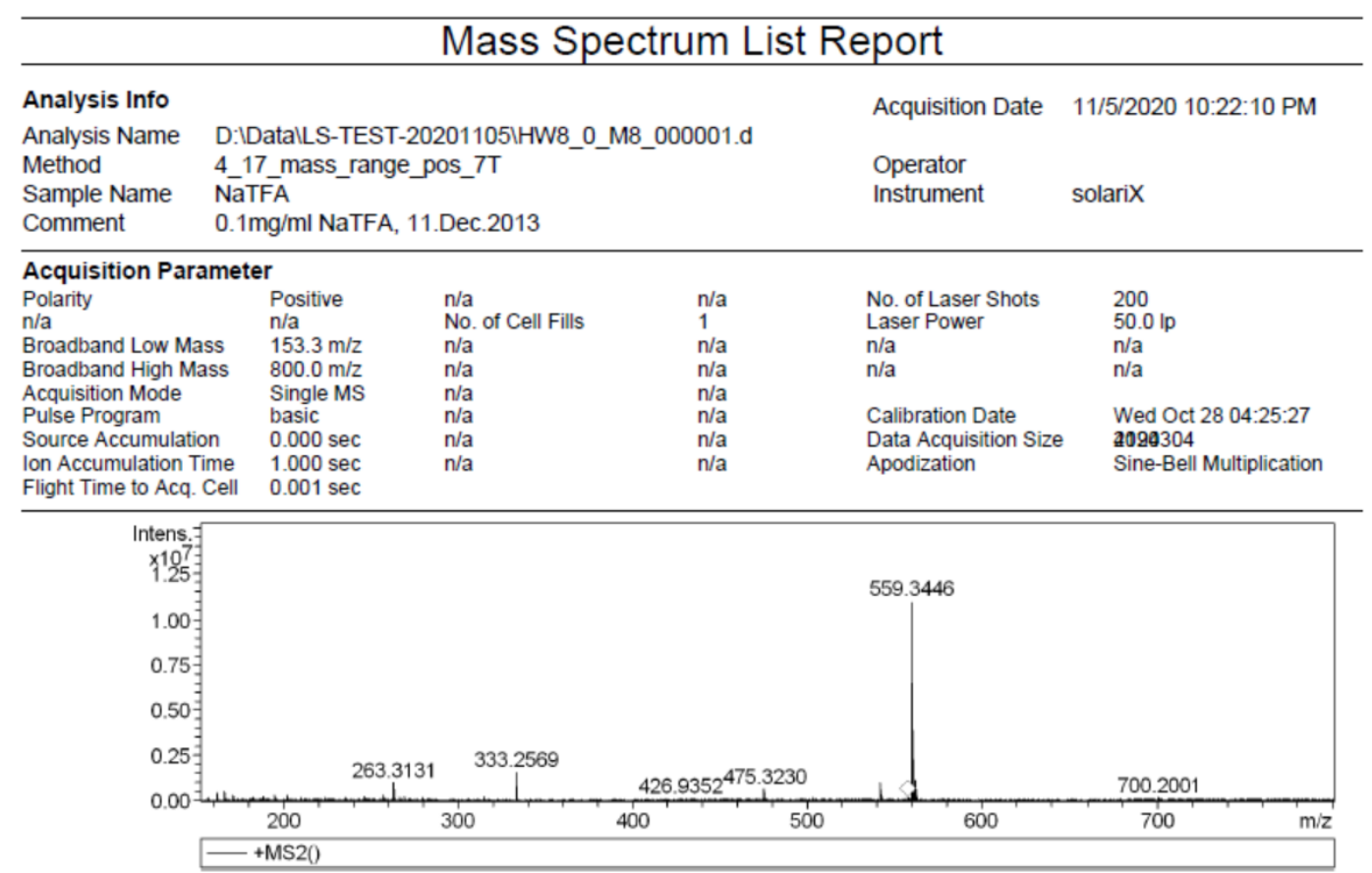

Figure S 2-3 HRESIMS Spectrum of compound 2b 
〈色谱图〉

$\mathrm{mV}$

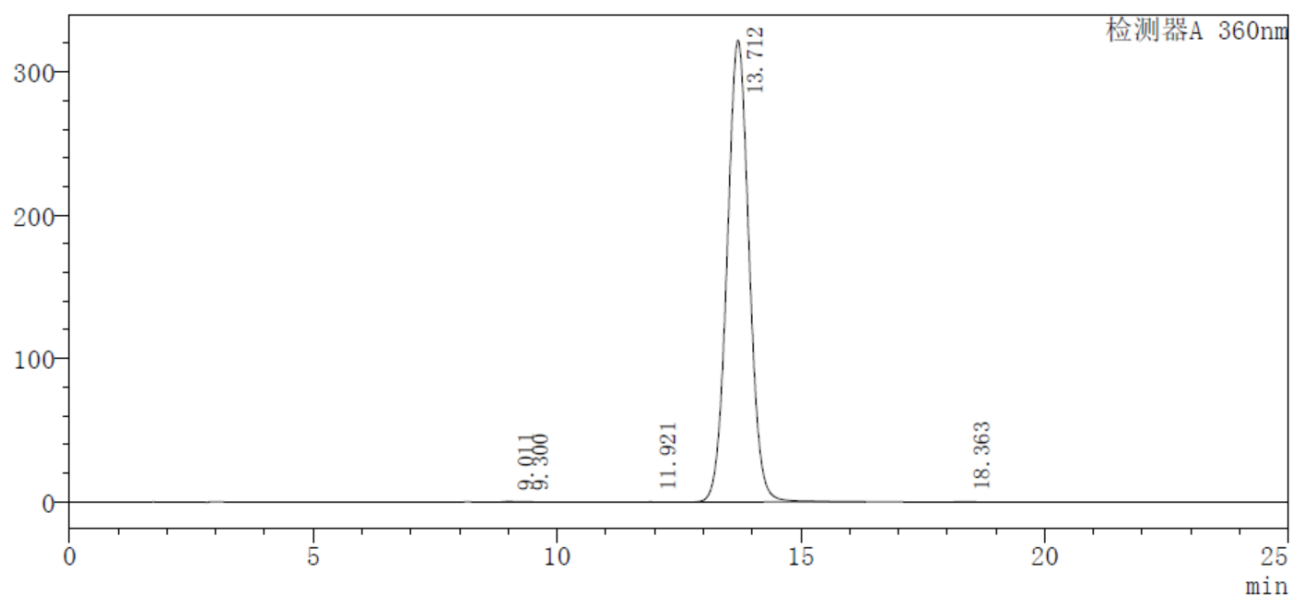

Figure S 2-4 HPLC Spectrum of compound 2b

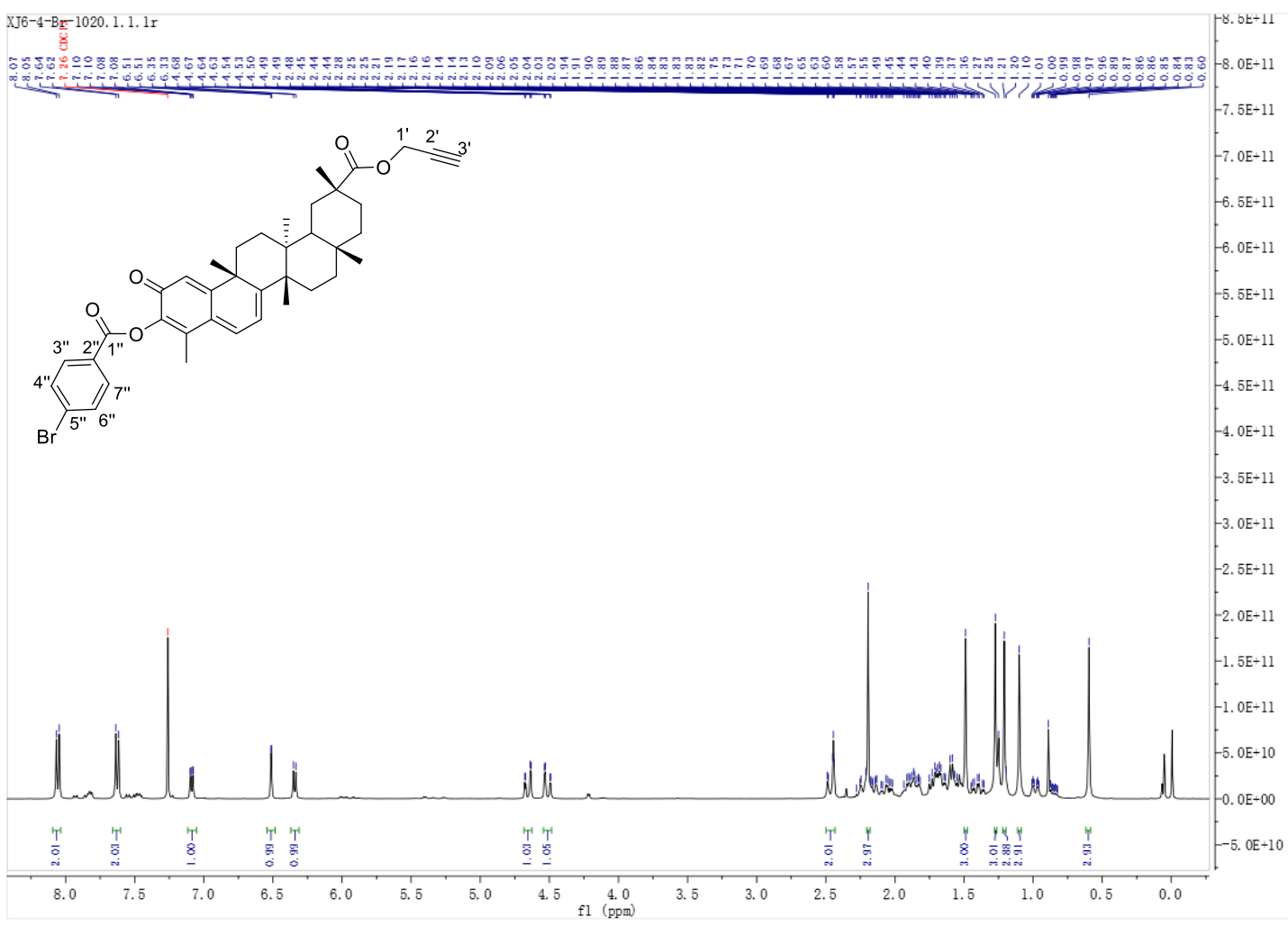

Figure S 3-1 ${ }^{1} \mathrm{H}$ NMR Spectrum (400MHz) of compound 2 c in $\mathrm{CDCl}_{3}$ 


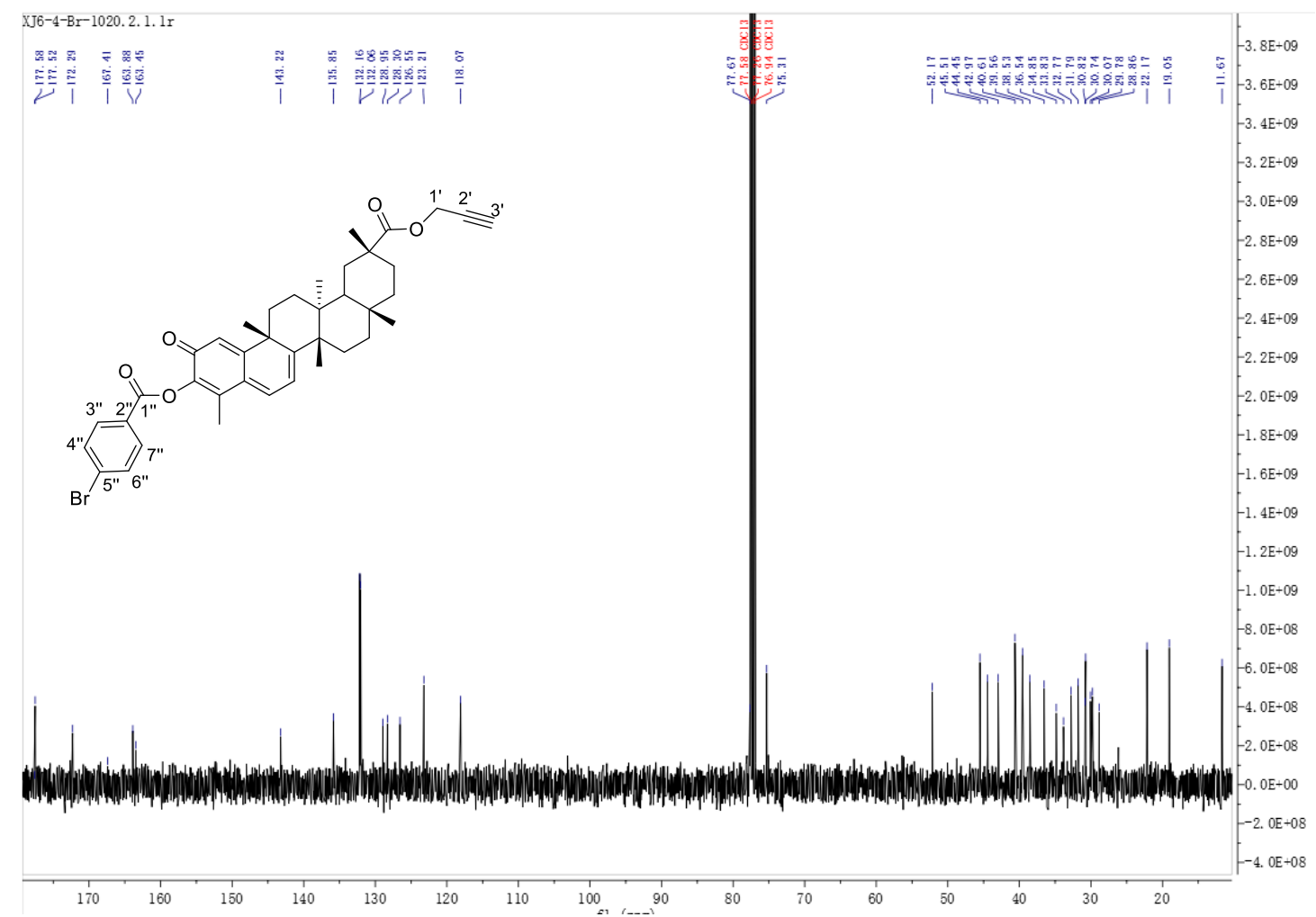

Figure S 3-2 ${ }^{13} \mathrm{C}$ NMR Spectrum $(100 \mathrm{MHz})$ of compound $2 \mathrm{c}$ in $\mathrm{CDCl}_{3}$

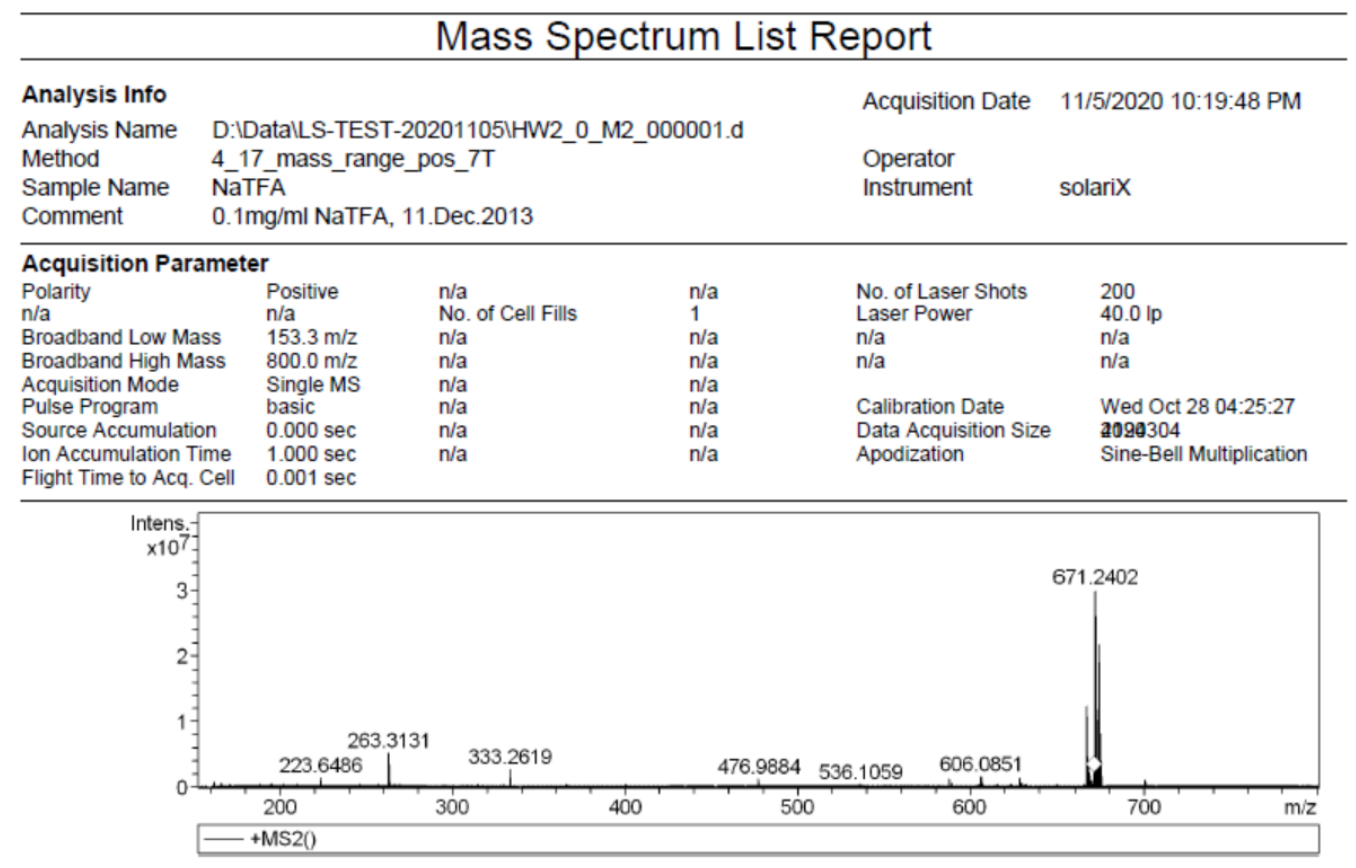

Figure S 3-3 HRESIMS Spectrum of 2c 


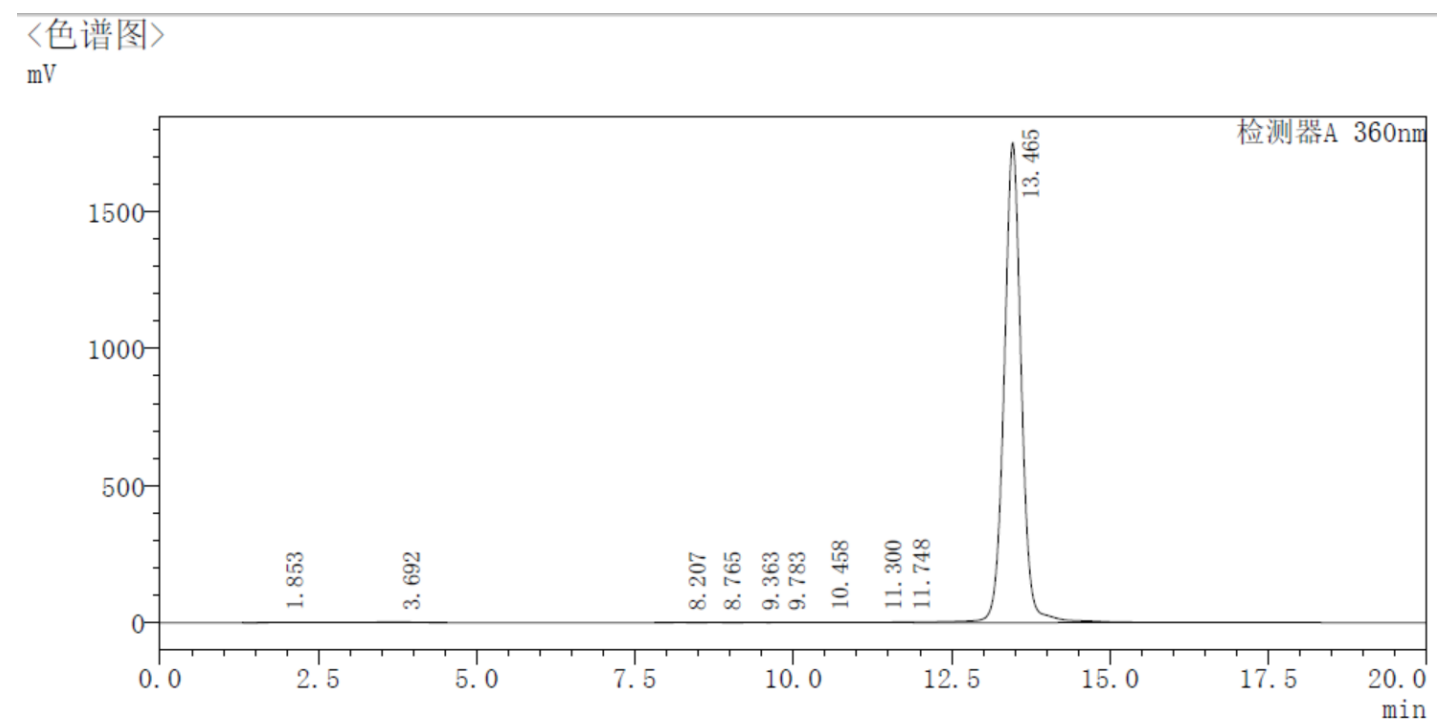

Figure S 3-4 HPLC Spectrum of 2c

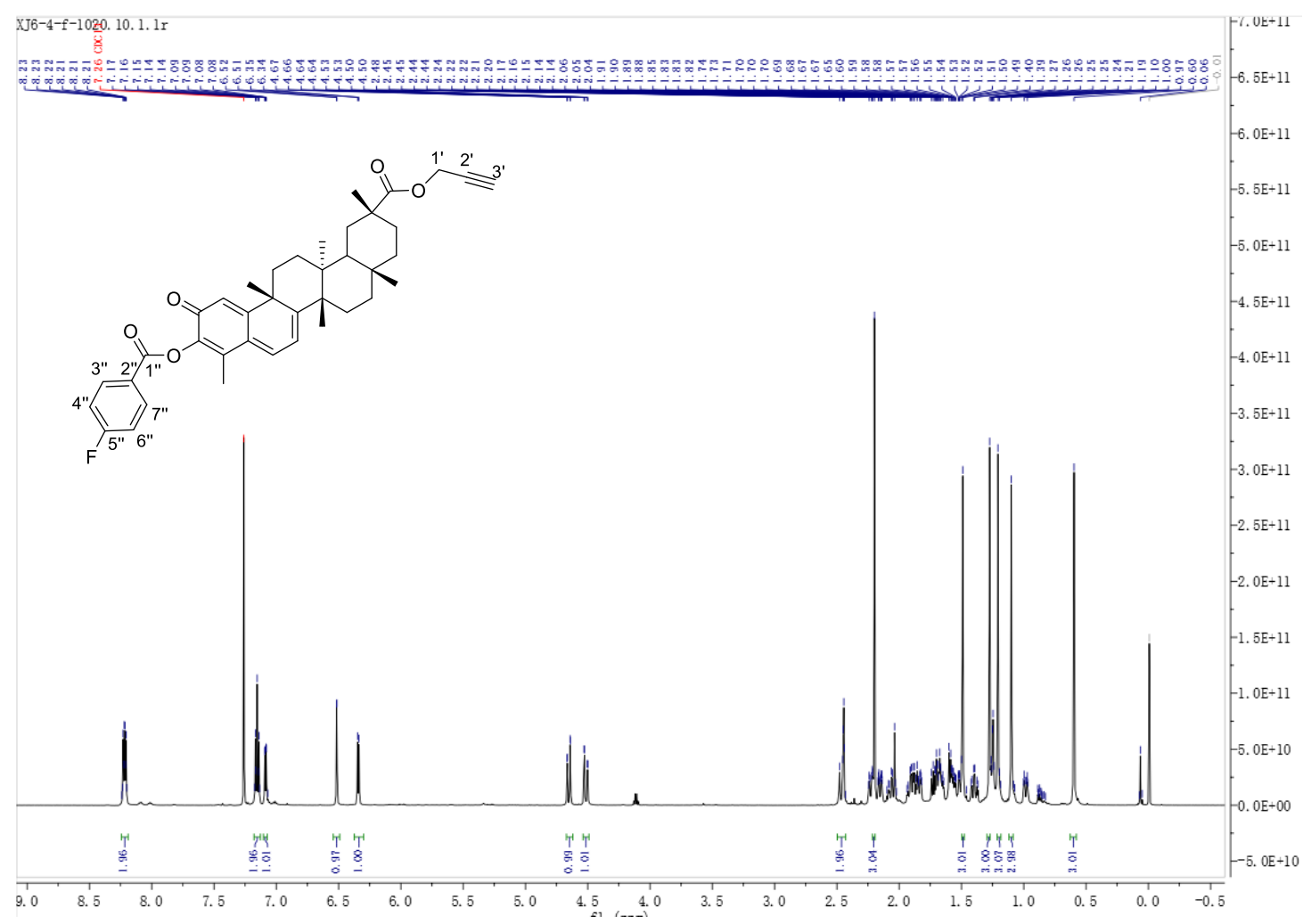

Figure S 4-1 ${ }^{1} \mathrm{H}$ NMR Spectrum $(600 \mathrm{MHz})$ of compound $2 \mathbf{d}$ in $\mathrm{CDCl}_{3}$ 


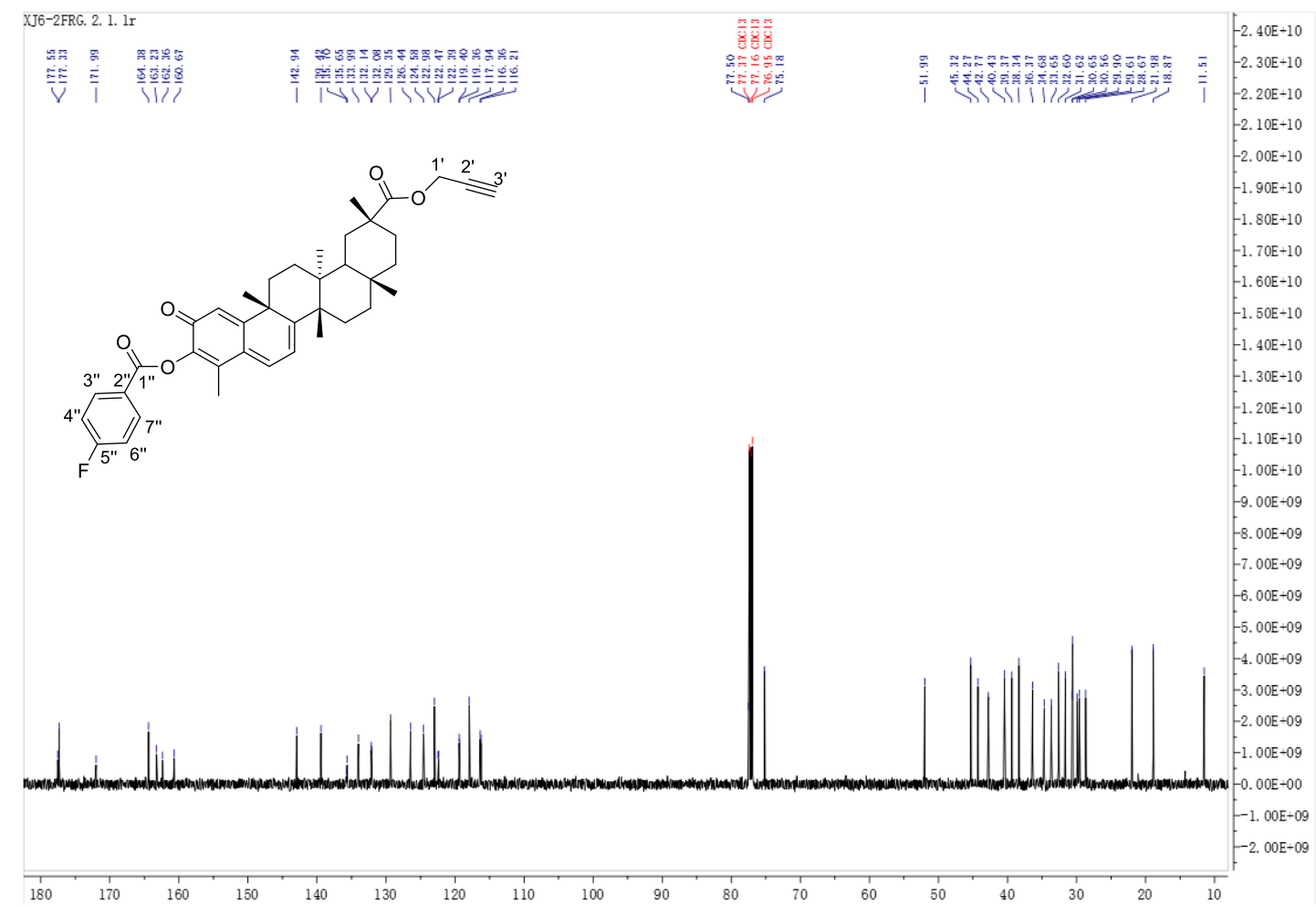

Figure S 4-2 ${ }^{13} \mathrm{C}$ NMR Spectrum $(150 \mathrm{MHz})$ of compound $\mathbf{2 d}$ in $\mathrm{CDCl}_{3}$

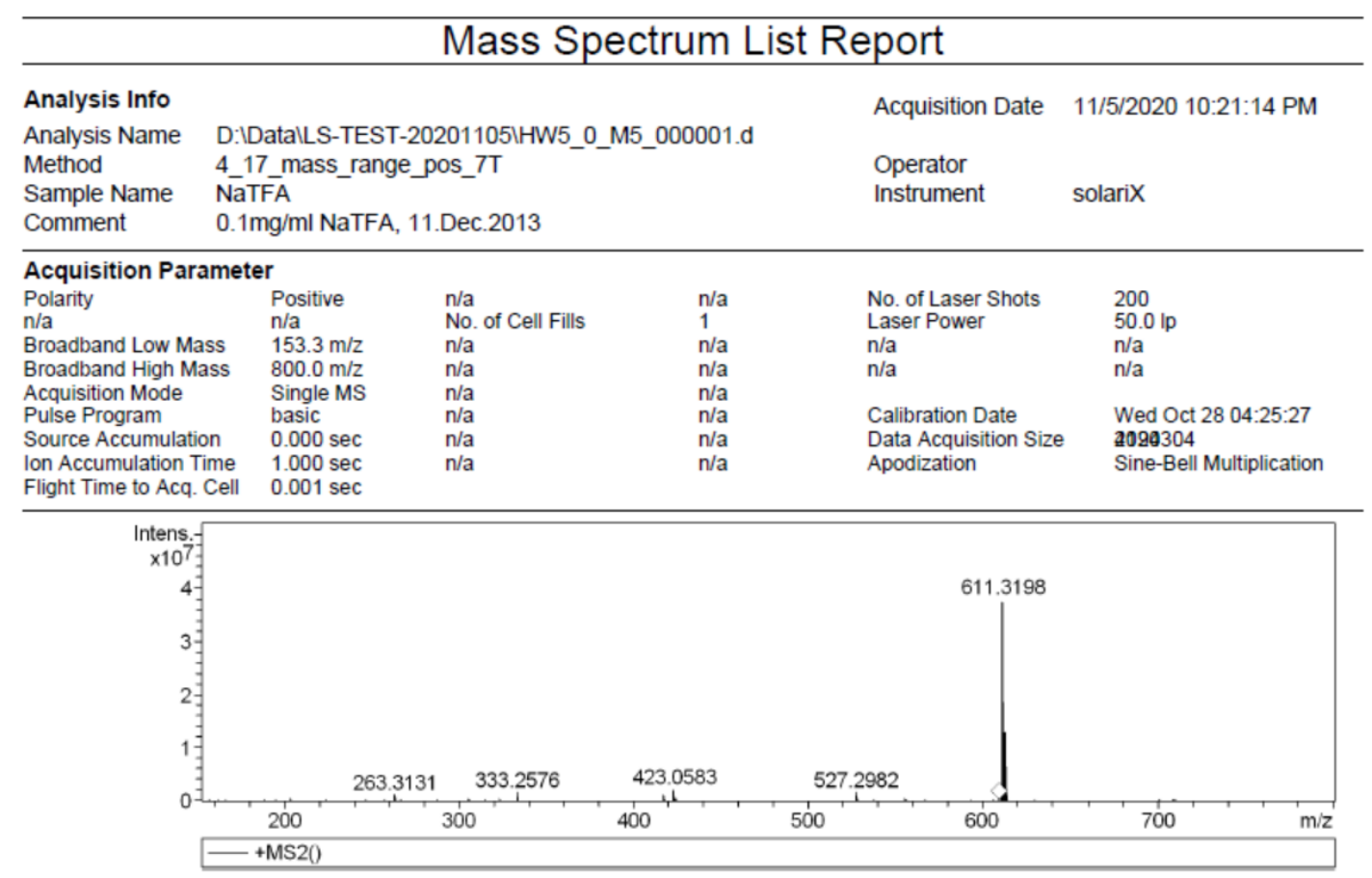

Figure S 4-3 HRESIMS Spectrum of 2d 


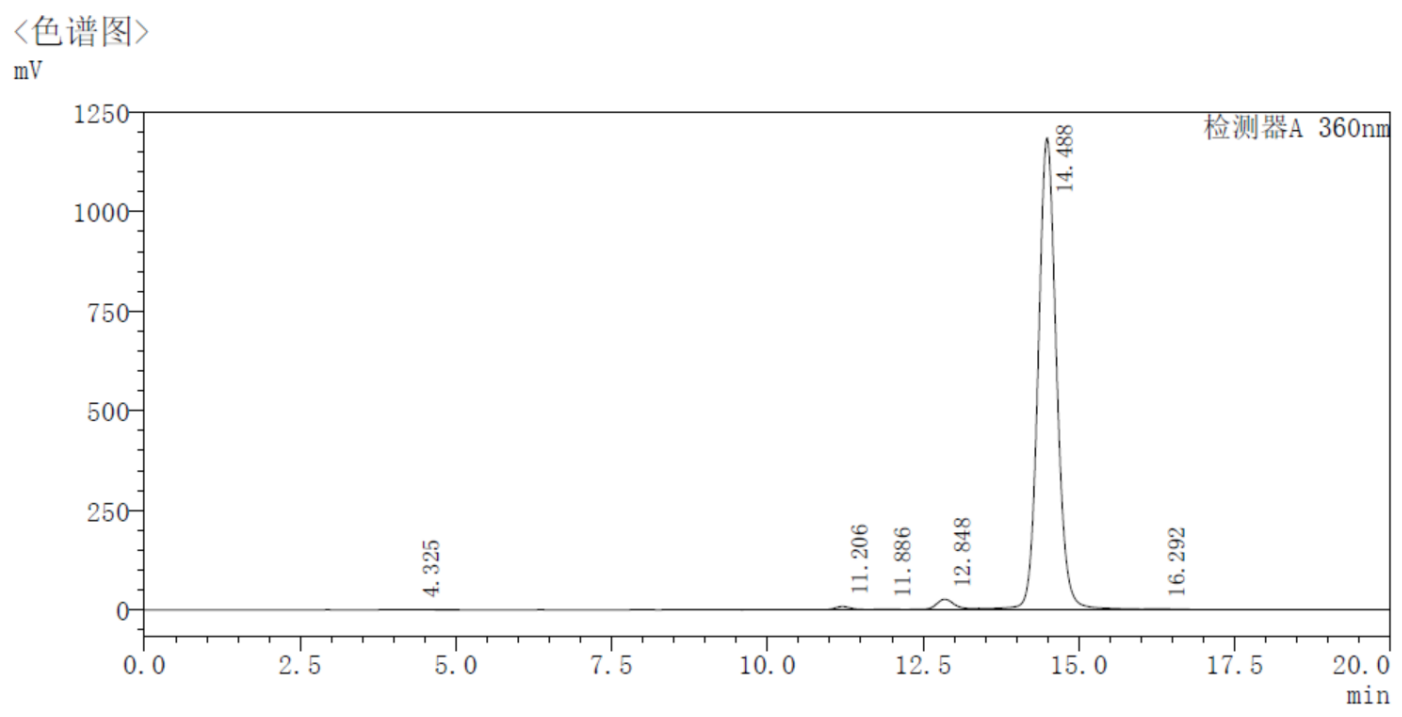

Figure S 4-4 HPLC Spectrum of 2d

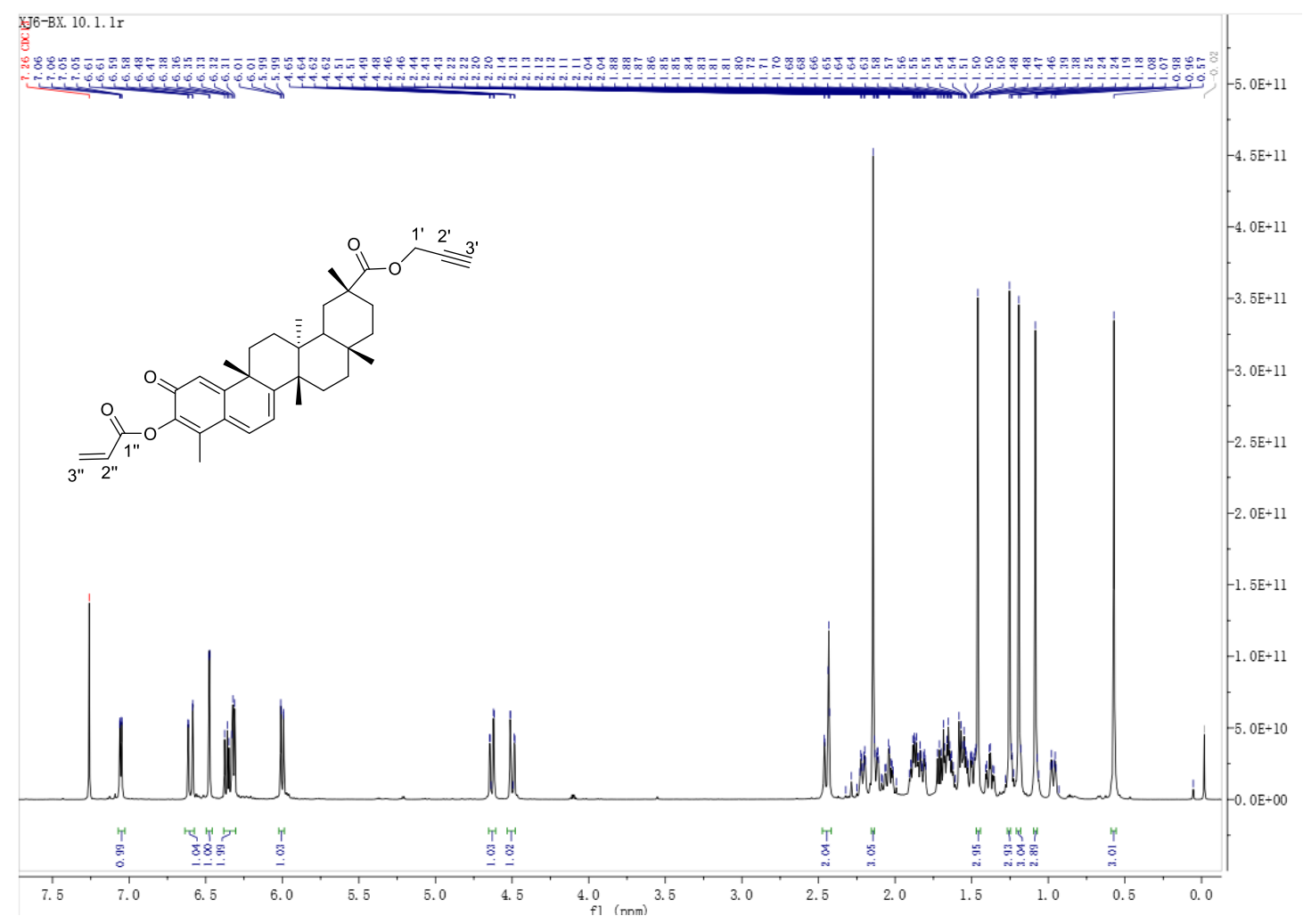

Figure S 5-1 ${ }^{1} \mathrm{H}$ NMR Spectrum $(600 \mathrm{MHz})$ of compound 3a in $\mathrm{CDCl}_{3}$ 


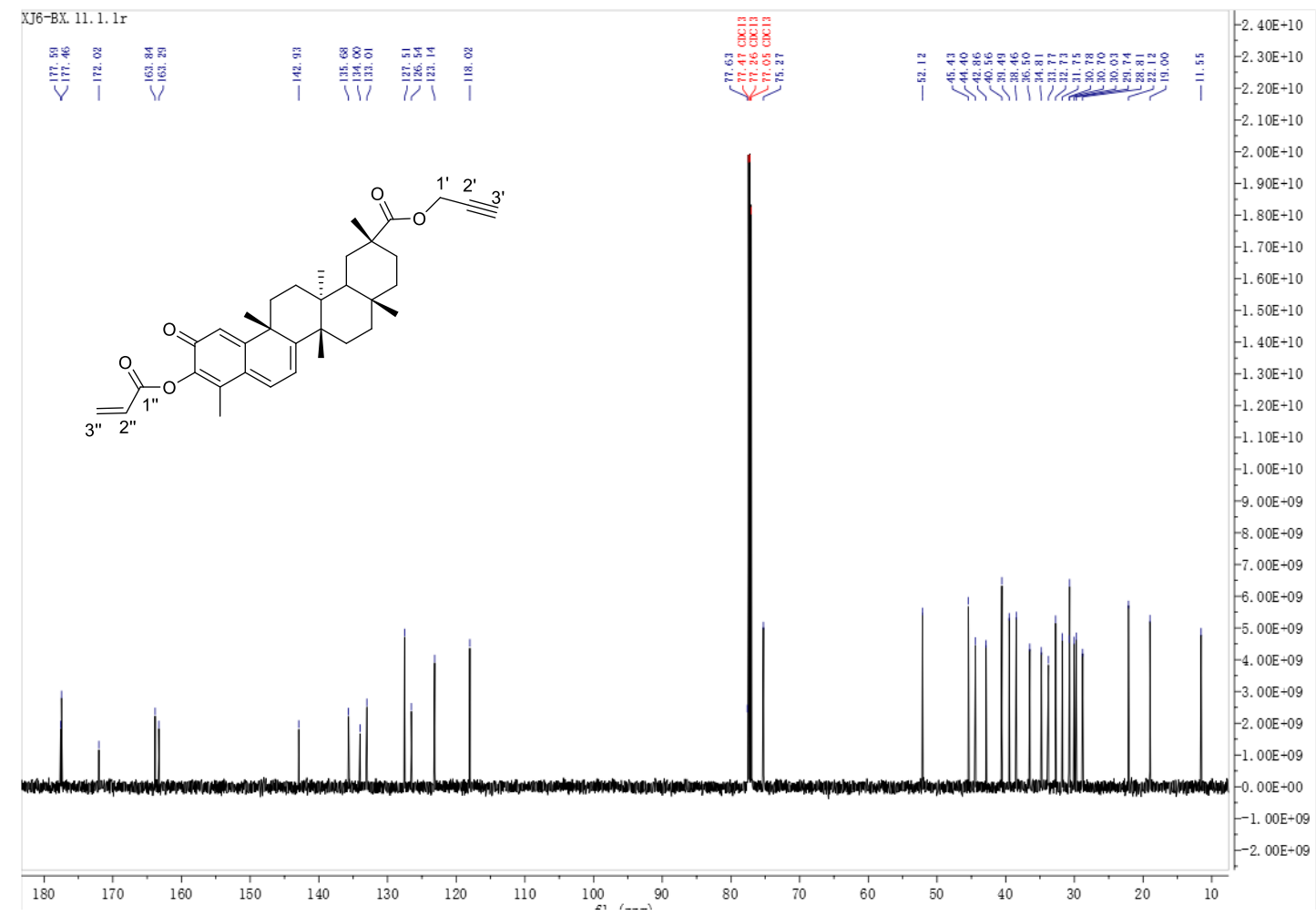

Figure S 5-2 ${ }^{13} \mathrm{C}$ NMR Spectrum $(150 \mathrm{MHz})$ of compound $\mathbf{3 a}$ in $\mathrm{CDCl}_{3}$

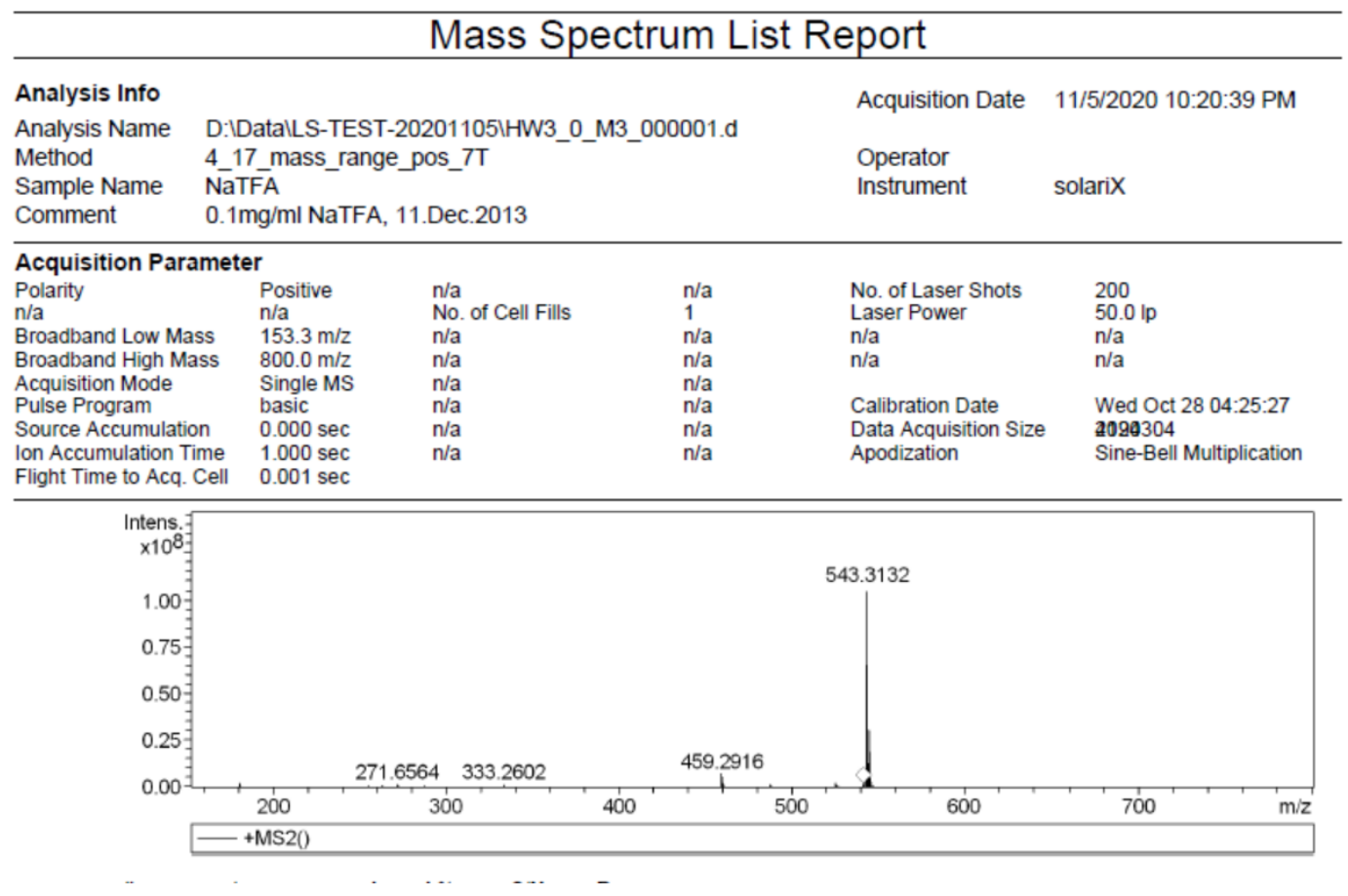

Figure S 5-3 HRESIMS Spectrum of 3a 
〈色谱图〉

$\mathrm{mV}$

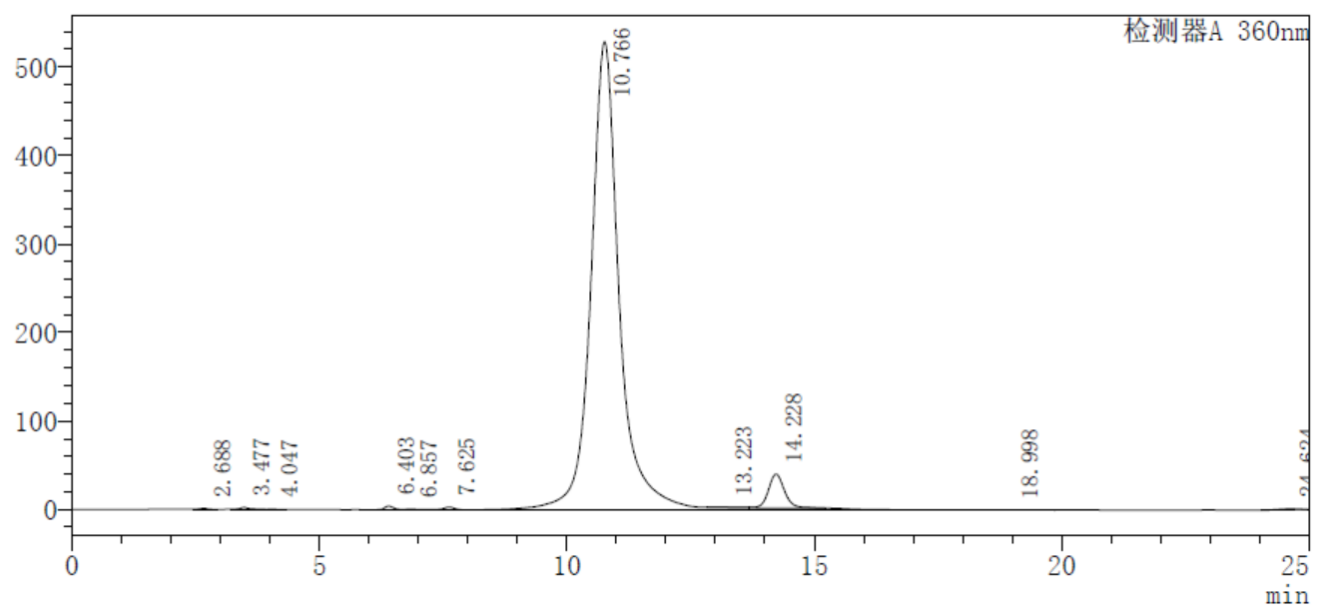

Figure S 5-4 HPLC Spectrum of 3a

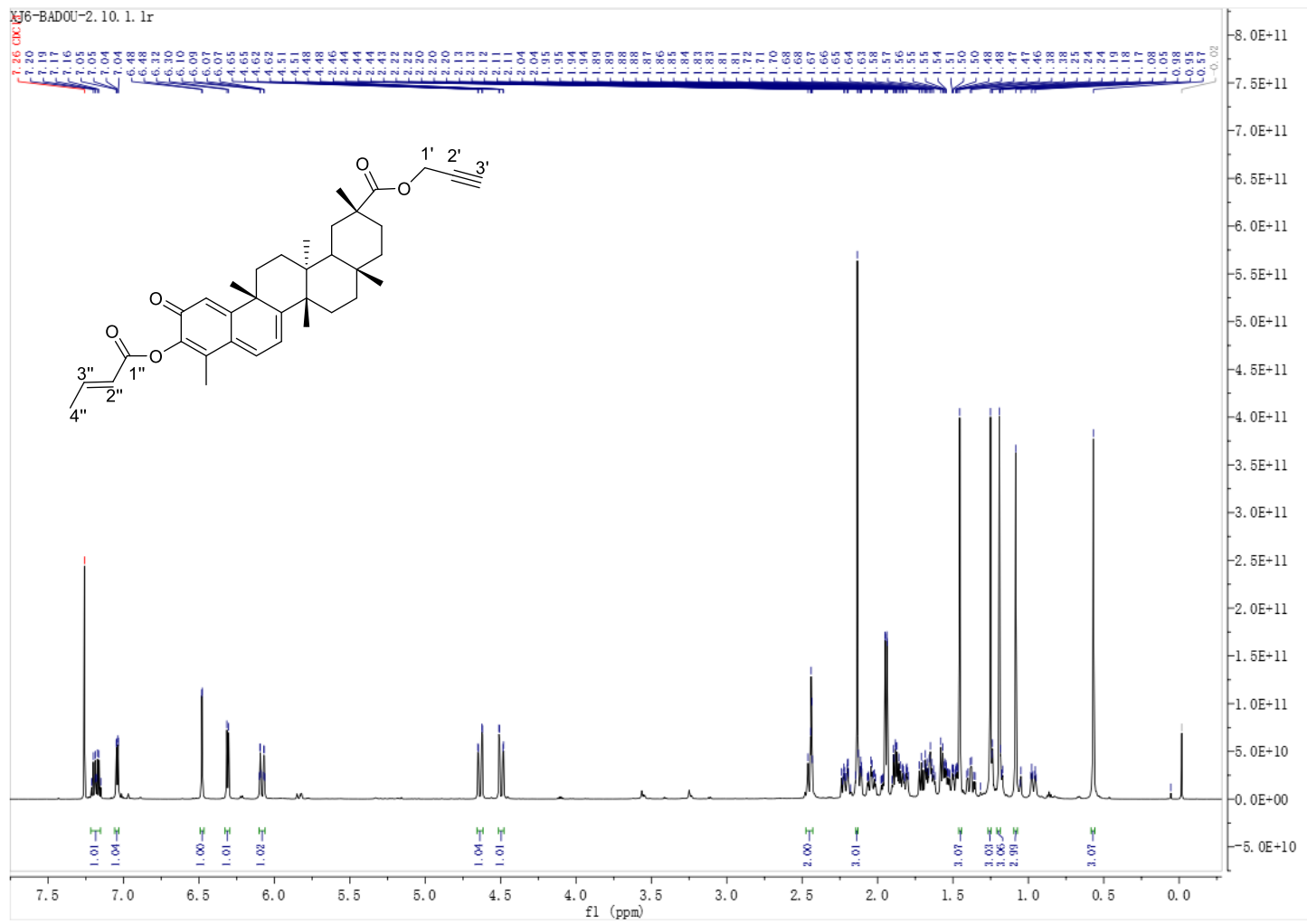

Figure S 6-1 ${ }^{1} \mathrm{H}$ NMR Spectrum $(400 \mathrm{MHz})$ of compound $\mathbf{3 b}$ in $\mathrm{CDCl}_{3}$ 


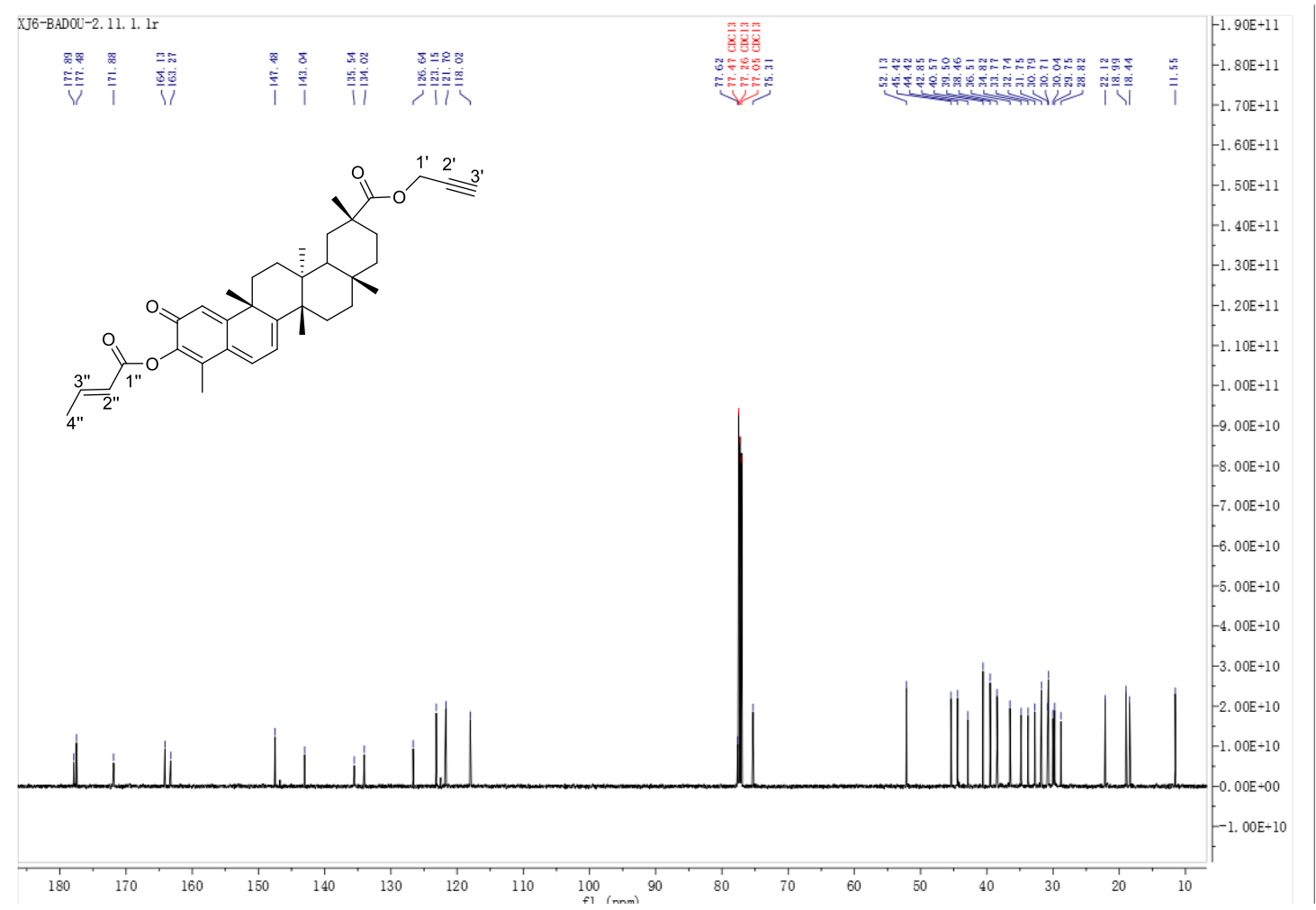

Figure S 6-2 ${ }^{13} \mathrm{C}$ NMR Spectrum $(100 \mathrm{MHz})$ of compound $\mathbf{3 b}$ in $\mathrm{CDCl}_{3}$

\begin{tabular}{|c|c|c|c|c|c|c|}
\hline \multicolumn{7}{|c|}{ Mass Spectrum List Report } \\
\hline $\begin{array}{l}\text { Analysis Info } \\
\text { Analysis Name } \\
\text { Method } \\
\text { Sample Name } \\
\text { Comment }\end{array}$ & $\begin{array}{l}\text { D:ID } \\
4 \_1 \\
\text { NaT } \\
0.1 \mathrm{n}\end{array}$ & $\begin{array}{l}\text { atalLS-TEST-202 } \\
\text { 7_mass_range_po } \\
\text { hy } / \text { ml NaTFA, } 11 . \mathrm{D}\end{array}$ & $\begin{array}{l}\text { 011051HW1_0_M1_- } \\
\text { os_7T } \\
\text { Dec.2013 }\end{array}$ & 001.d & $\begin{array}{l}\text { Acquisition Date } \\
\text { Operator } \\
\text { Instrument }\end{array}$ & solariX \\
\hline $\begin{array}{l}\text { Acquisition Paran } \\
\text { Polarity } \\
\text { n/a } \\
\text { Broadband Low Mas } \\
\text { Broadband High Mas } \\
\text { Acquisition Mode } \\
\text { Pulse Program } \\
\text { Source Accumulation } \\
\text { Ion Accumulation Tim } \\
\text { Flight Time to Acq. C }\end{array}$ & $\begin{array}{l}\text { ss } \\
\text { iss } \\
\text { in } \\
\text { me } \\
\text { Cell }\end{array}$ & $\begin{array}{l}\text { Positive } \\
\text { n/a } \\
153.3 \mathrm{~m} / \mathrm{z} \\
800.0 \mathrm{~m} / \mathrm{z} \\
\text { Single MS } \\
\text { basic } \\
0.000 \mathrm{sec} \\
1.000 \mathrm{sec} \\
0.001 \mathrm{sec}\end{array}$ & $\begin{array}{l}n / a \\
\text { No. of Cell Fills } \\
\text { n/a } \\
n / a \\
n / a \\
n / a \\
n / a \\
n / a\end{array}$ & $\begin{array}{l}\mathrm{n} / \mathrm{a} \\
1 \\
\mathrm{n} / \mathrm{a} \\
\mathrm{n} / \mathrm{a} \\
\mathrm{n} / \mathrm{a} \\
\mathrm{n} / \mathrm{a} \\
\mathrm{n} / \mathrm{a} \\
\mathrm{n} / \mathrm{a}\end{array}$ & $\begin{array}{l}\text { No. of Laser Shots } \\
\text { Laser Power } \\
\text { n/a } \\
\text { n/a } \\
\text { Calibration Date } \\
\text { Data Acquisition Size } \\
\text { Apodization }\end{array}$ & $\begin{array}{l}200 \\
40.0 \text { lp } \\
\text { n/a } \\
\text { n/a } \\
\text { Wed Oct } 28 \text { 04:25:27 } \\
\text { 2090304 } \\
\text { Sine-Bell Multiplication }\end{array}$ \\
\hline 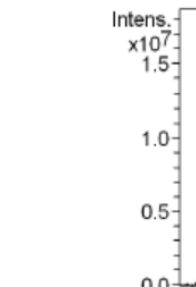 & & 263.3131 & 333.2588 & 473.3072 & 557.3289 & 700.1847 \\
\hline & & 200 & 400 & 500 & 600 & 700 \\
\hline
\end{tabular}

Figure S 6-3 HRESIMS Spectrum of 3b 
〈色谱图〉

$\mathrm{mV}$

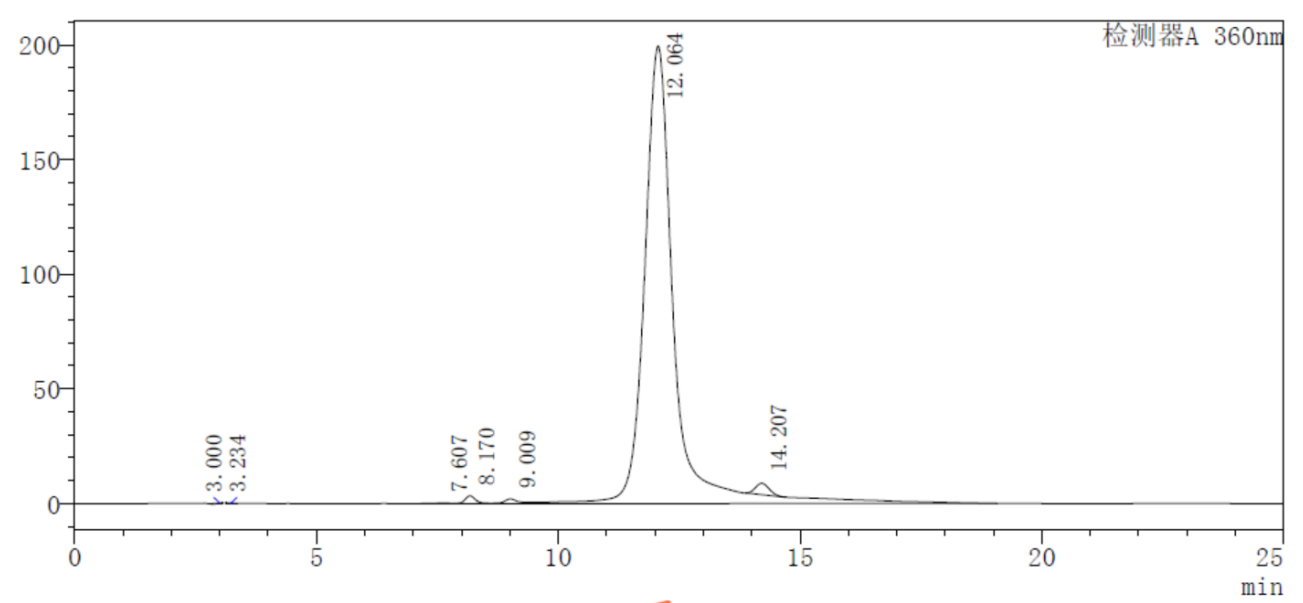

Figure S 6-4 HPLC Spectrum of 3b

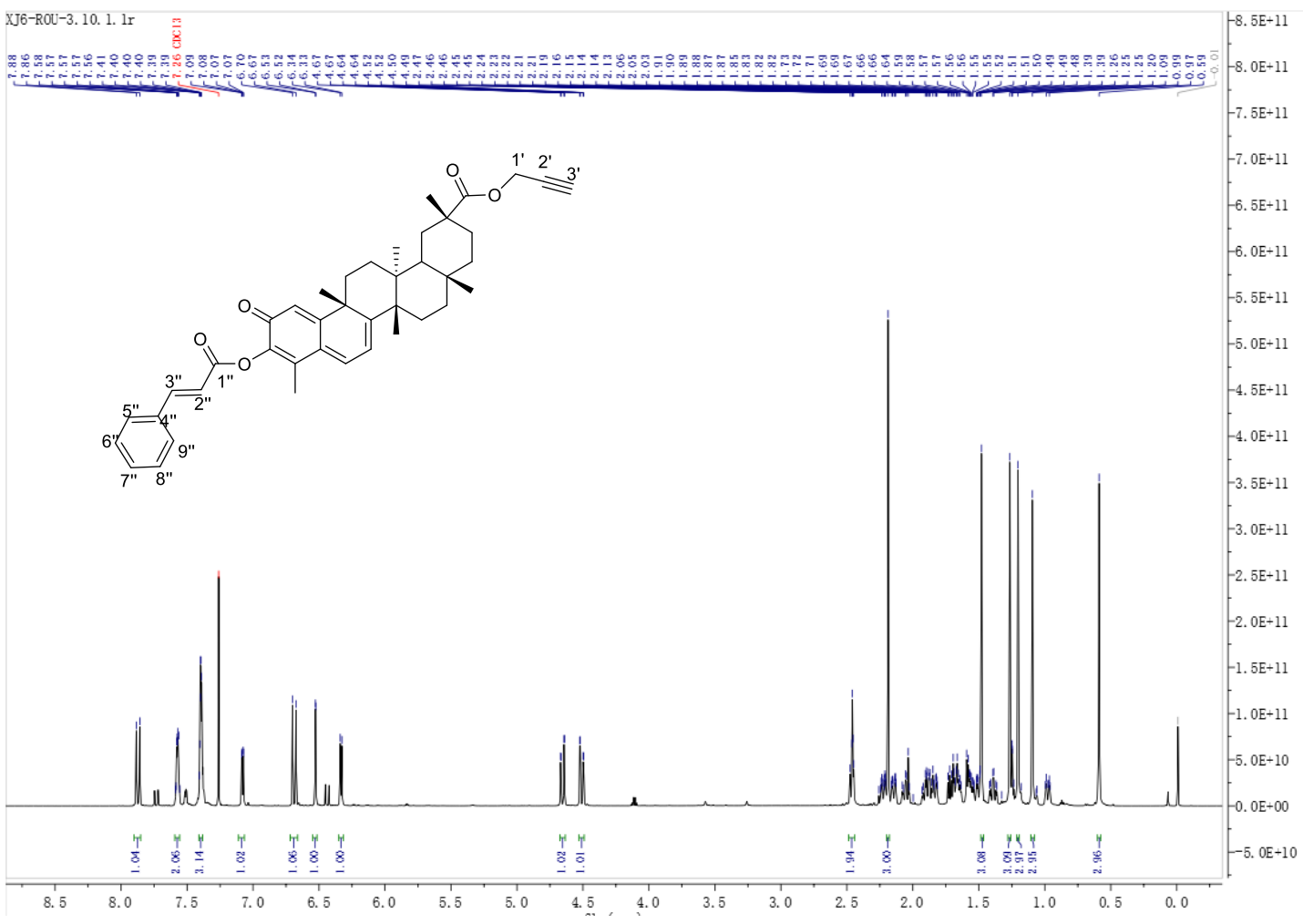

Figure S 7-1 ${ }^{1} \mathrm{H}$ NMR Spectrum $(600 \mathrm{MHz})$ of compound $3 \mathbf{c}$ in $\mathrm{CDCl}_{3}$ 


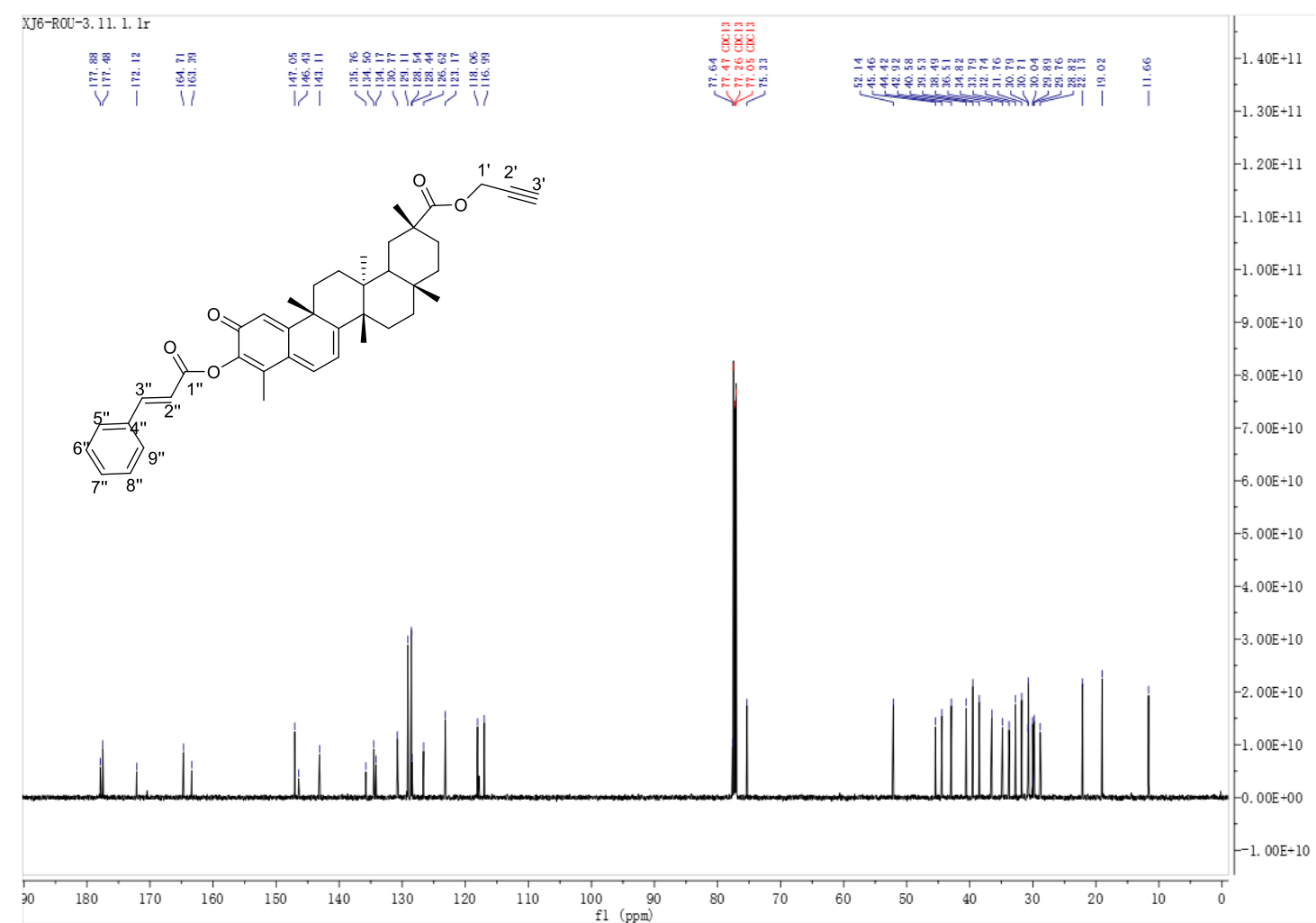

Figure S 7-2 ${ }^{13} \mathrm{C}$ NMR Spectrum $(150 \mathrm{MHz})$ of compound $\mathbf{3 c}$ in $\mathrm{CDCl}_{3}$

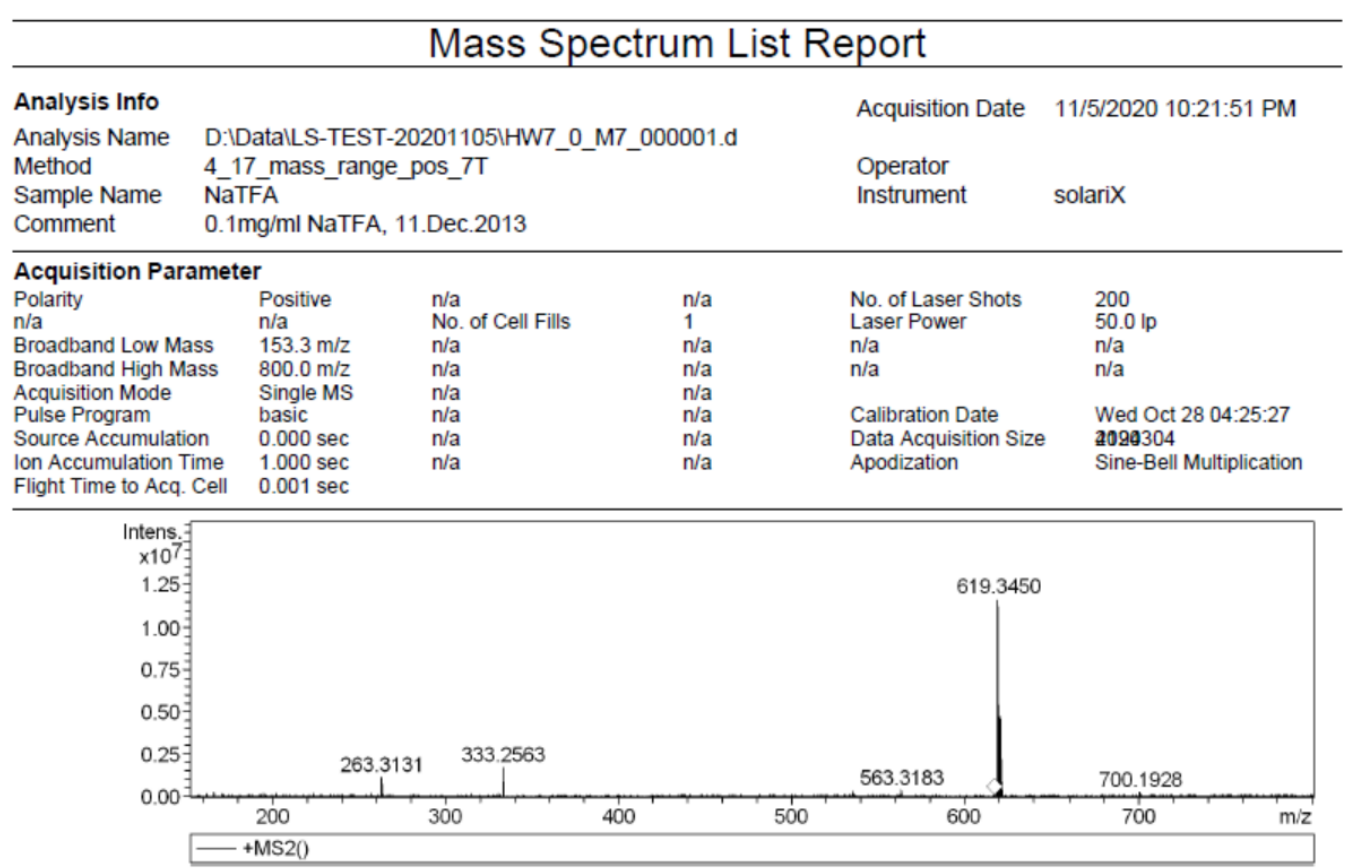

Figure S 7-3 HRESIMS Spectrum of 3c 
〈色谱图〉

$\mathrm{mV}$

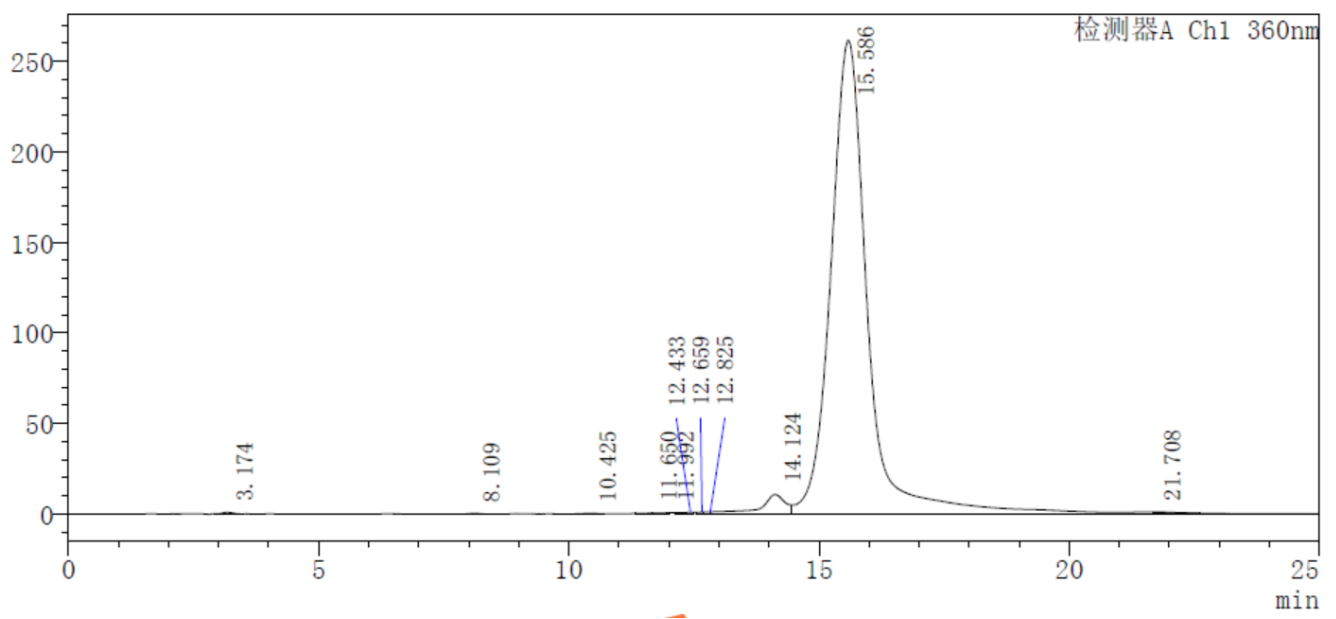

Figure S 7-4 HPLC Spectrum of 3c

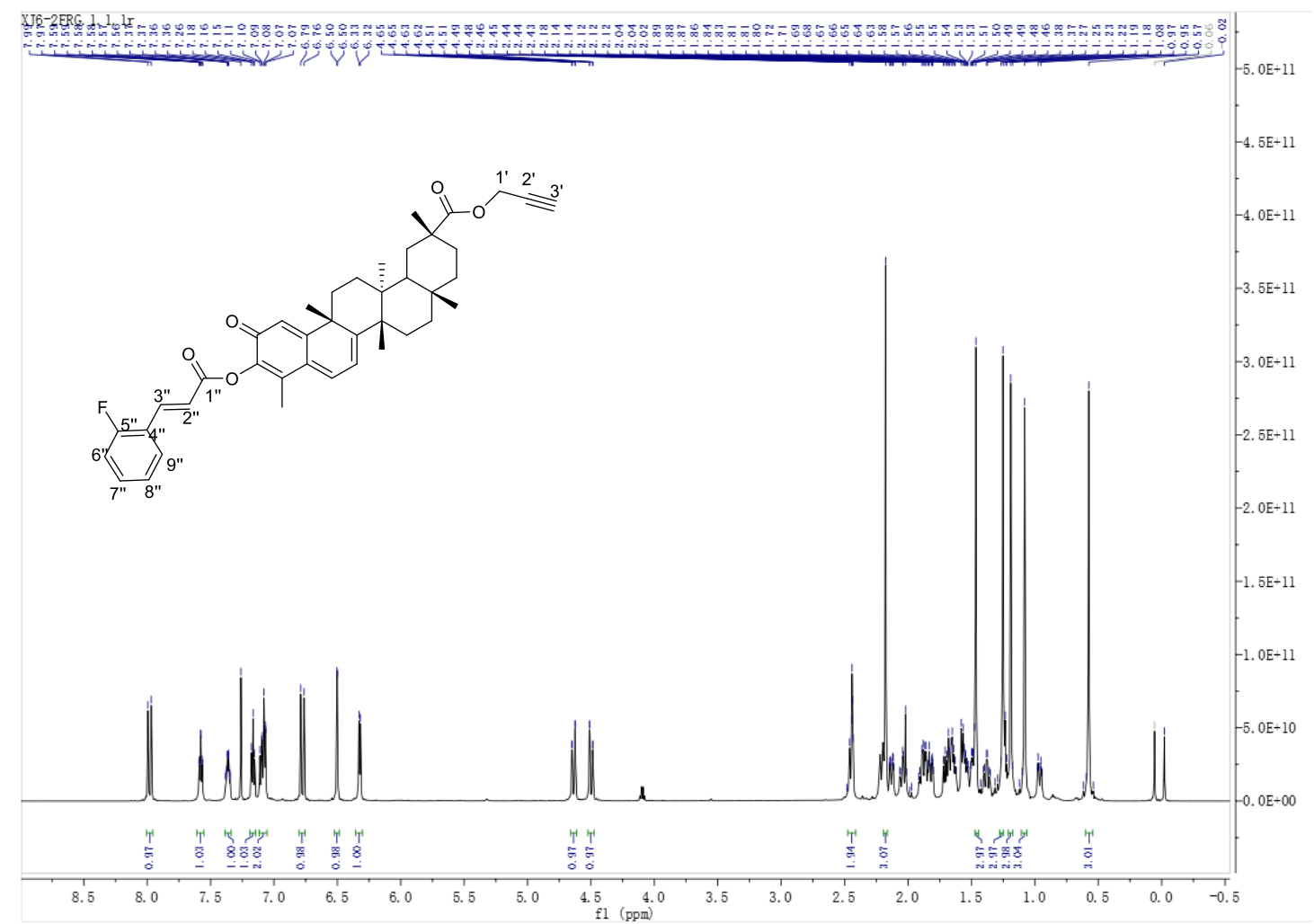

Figure S 8-1 ${ }^{1} \mathrm{H}$ NMR Spectrum $(600 \mathrm{MHz})$ of compound 3d in $\mathrm{CDCl}_{3}$ 


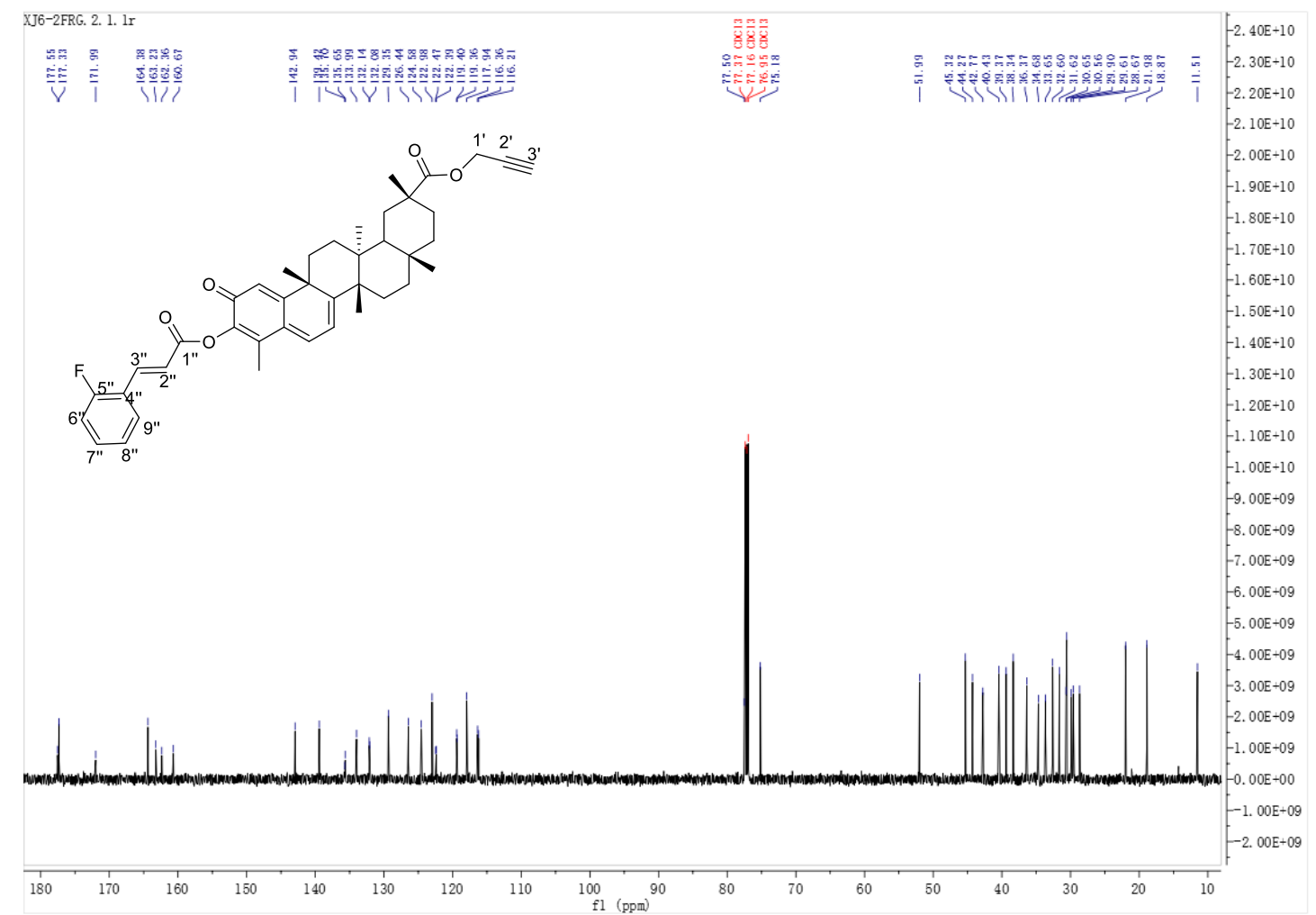

Figure S 8-2 ${ }^{13} \mathrm{C}$ NMR Spectrum $(150 \mathrm{MHz})$ of compound $\mathbf{3 d}$ in $\mathrm{CDCl}_{3}$

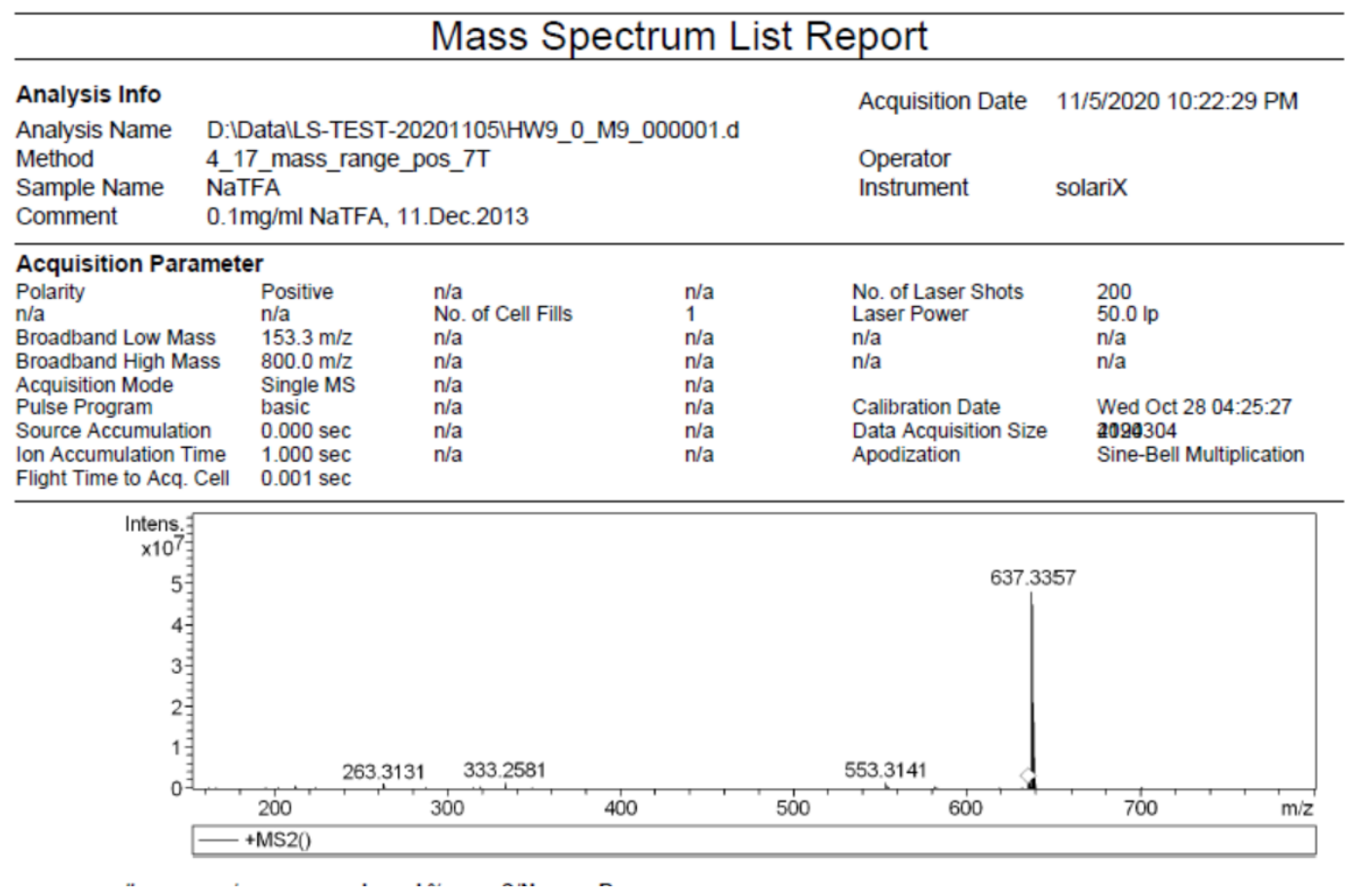

Figure S 8-3 HRESIMS Spectrum of 3d 
〈色谱图〉

$\mathrm{mV}$

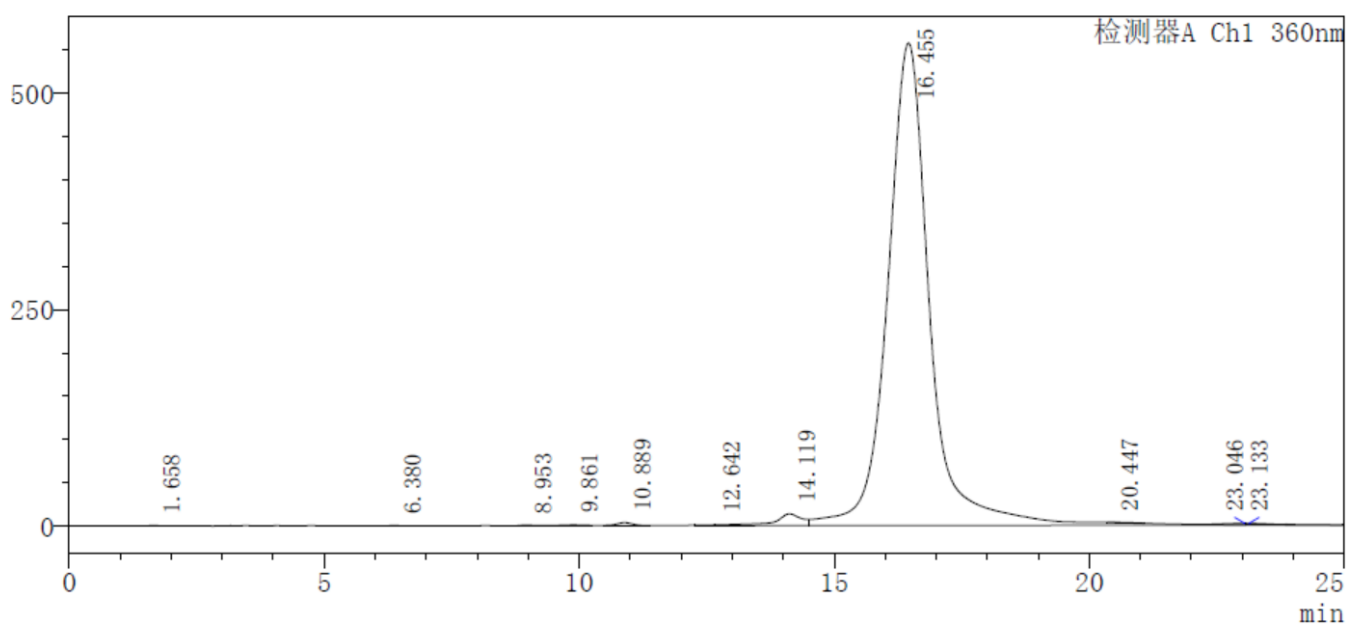

Figure S 8-4 HPLC Spectrum of 3d

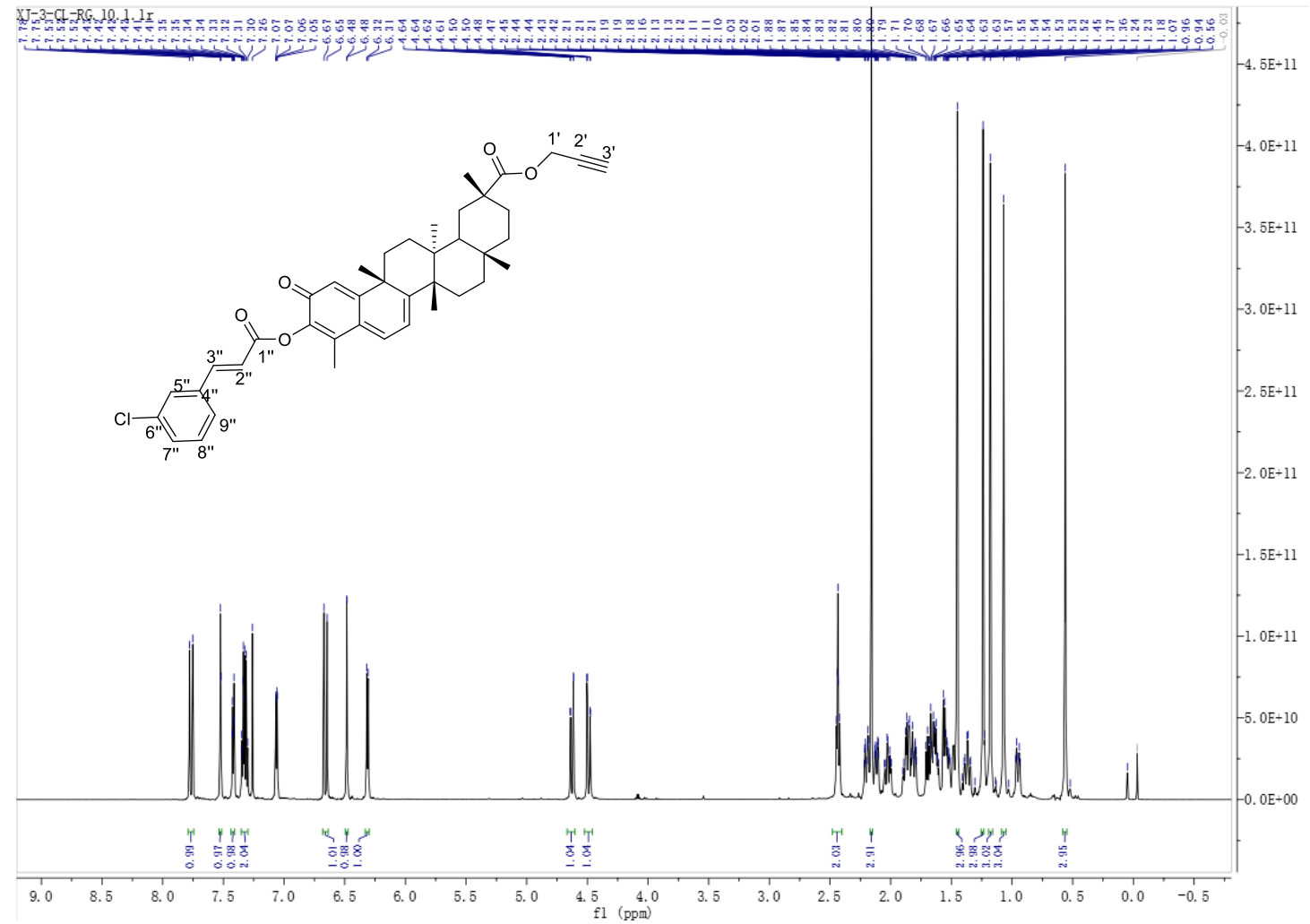

Figure S 9-1 ${ }^{1} \mathrm{H}$ NMR Spectrum $(600 \mathrm{MHz})$ of compound $3 \mathbf{e}$ in $\mathrm{CDCl}_{3}$ 


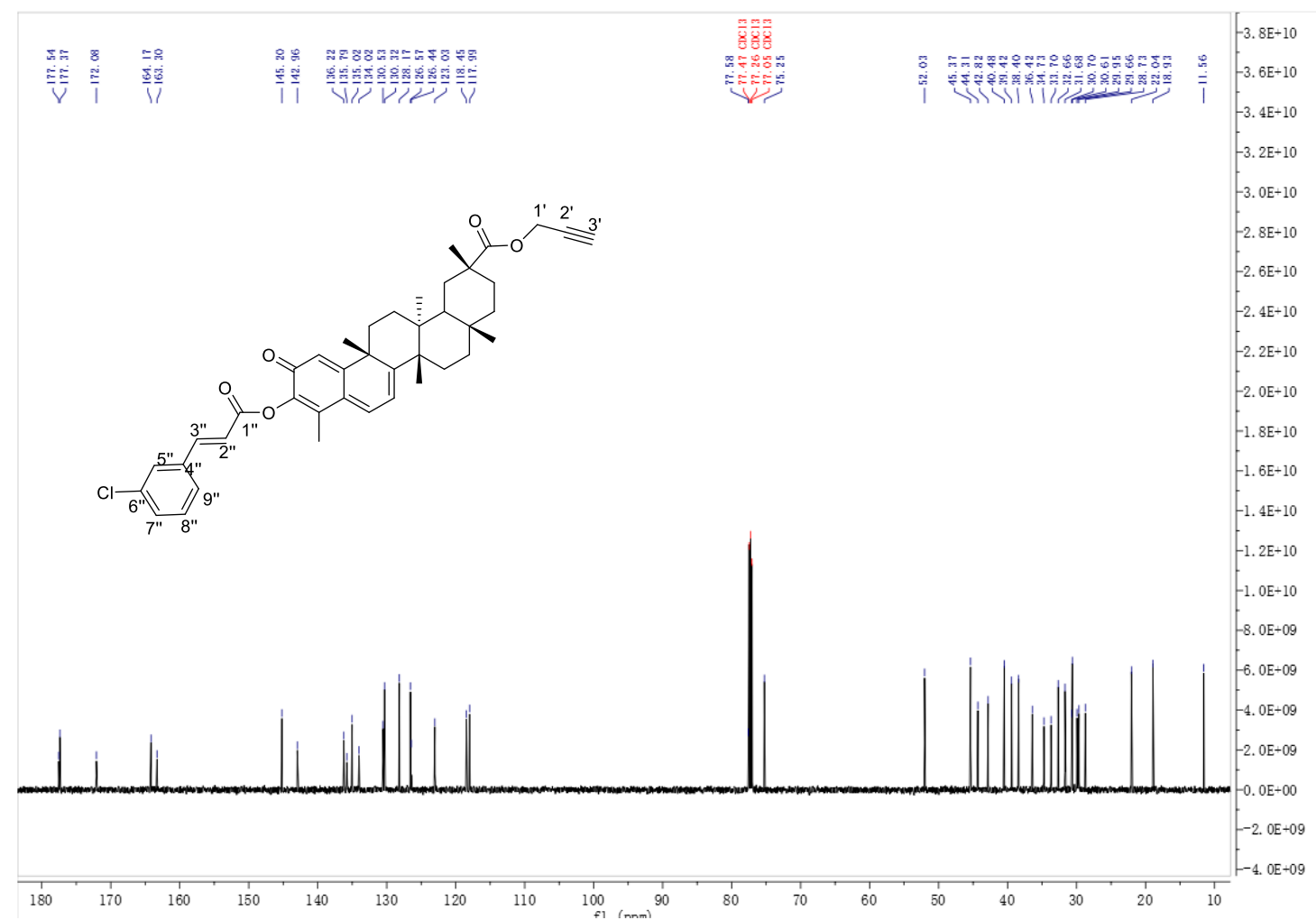

Figure S 9-2 ${ }^{13} \mathrm{C}$ NMR Spectrum $(150 \mathrm{MHz})$ of compound $3 \mathbf{e}$ in $\mathrm{CDCl}_{3}$

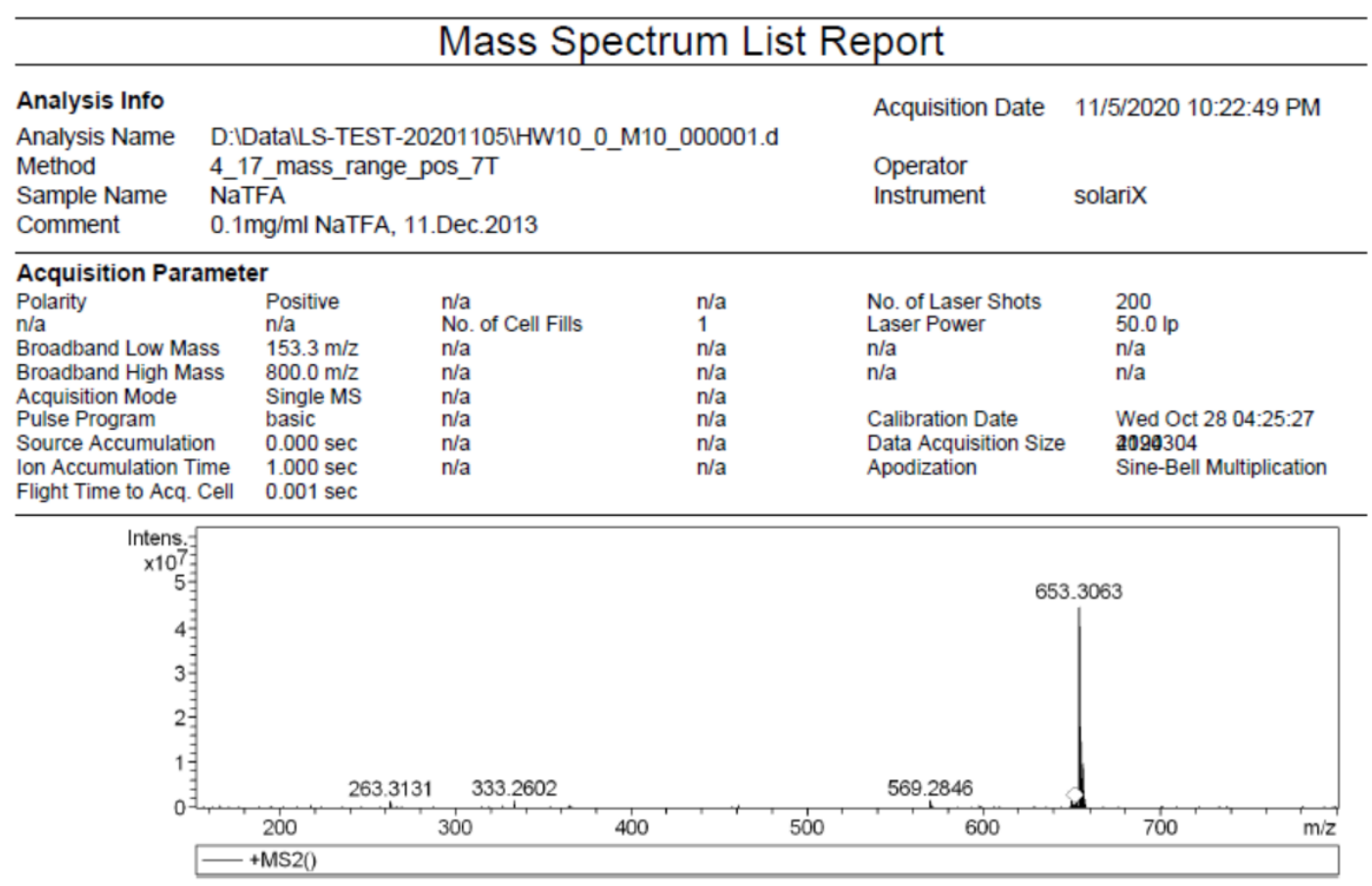

Figure S 9-3 HRESIMS Spectrum of 3e 
〈色谱图〉

$\mathrm{mV}$

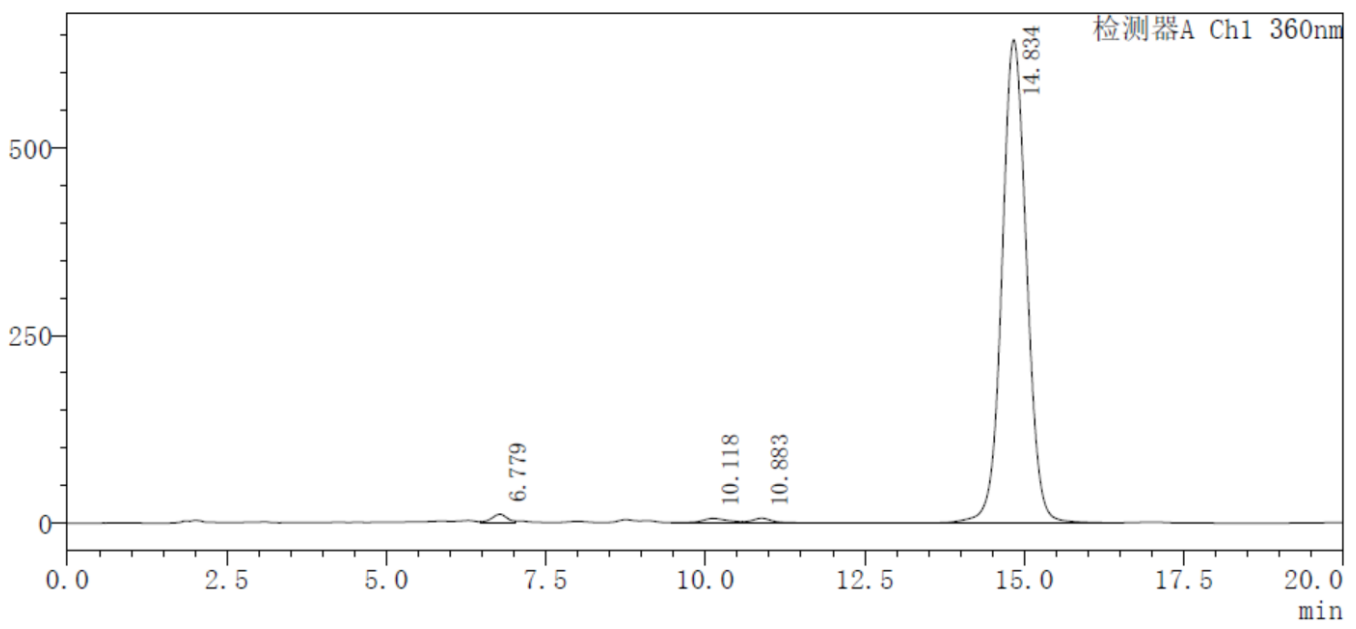

Figure S 9-4 HPLC Spectrum of 3e

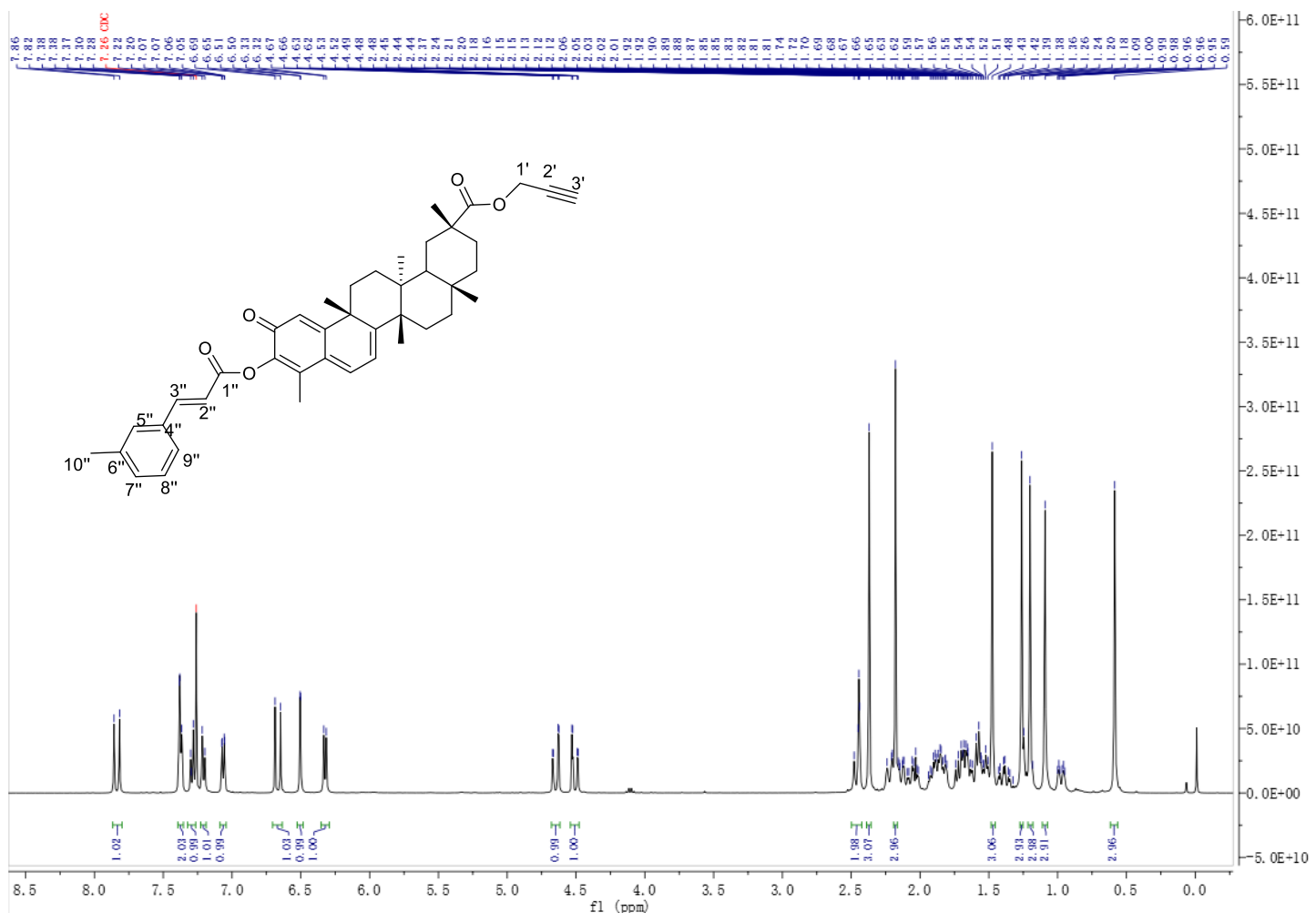

Figure S 10-1 ${ }^{1} \mathrm{H}$ NMR Spectrum (400MHz) of compound $\mathbf{3 f}$ in $\mathrm{CDCl}_{3}$ 


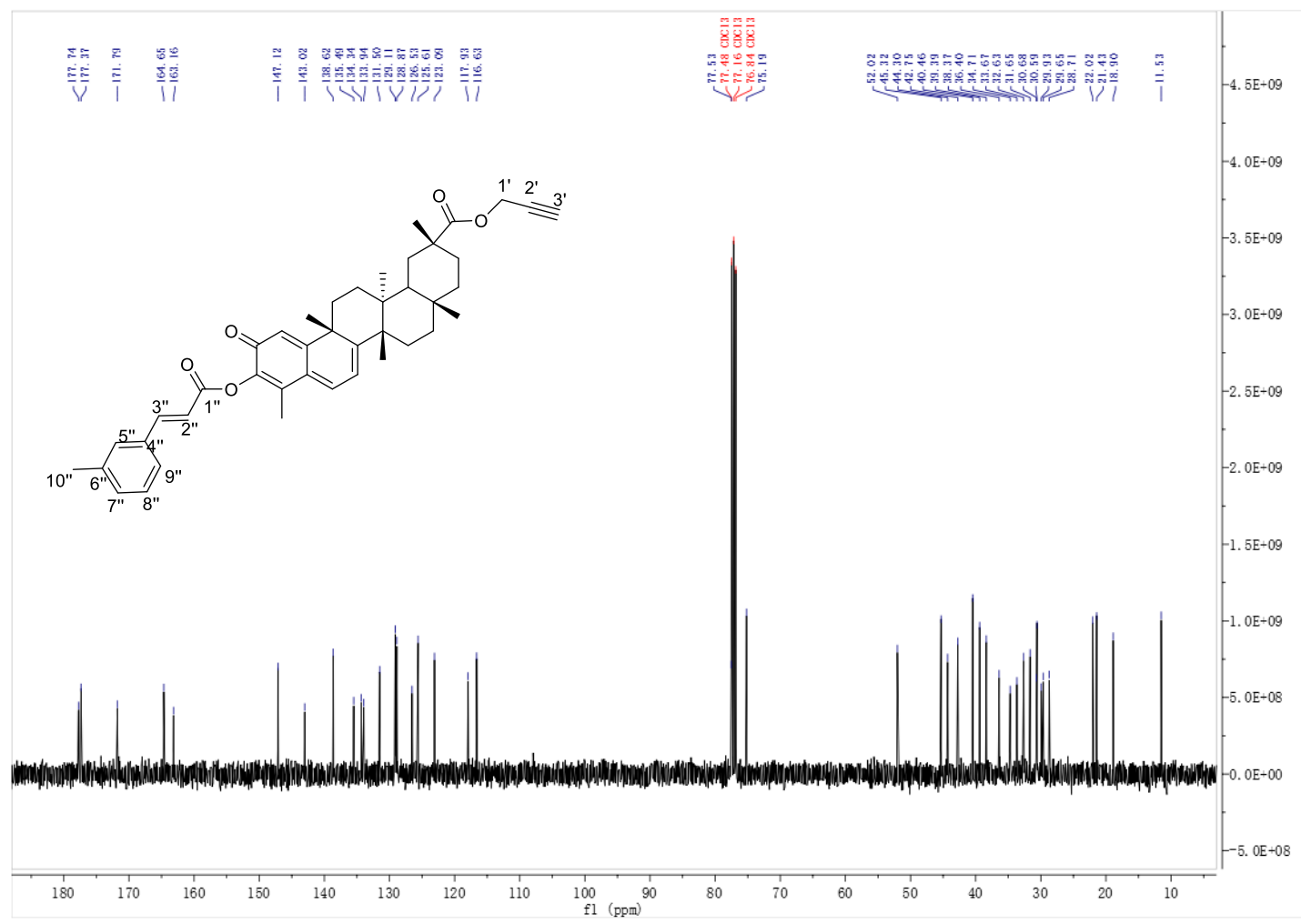

Figure S 10-2 ${ }^{13} \mathrm{C}$ NMR Spectrum $(100 \mathrm{MHz})$ of compound $\mathbf{3 f}$ in $\mathrm{CDCl}_{3}$

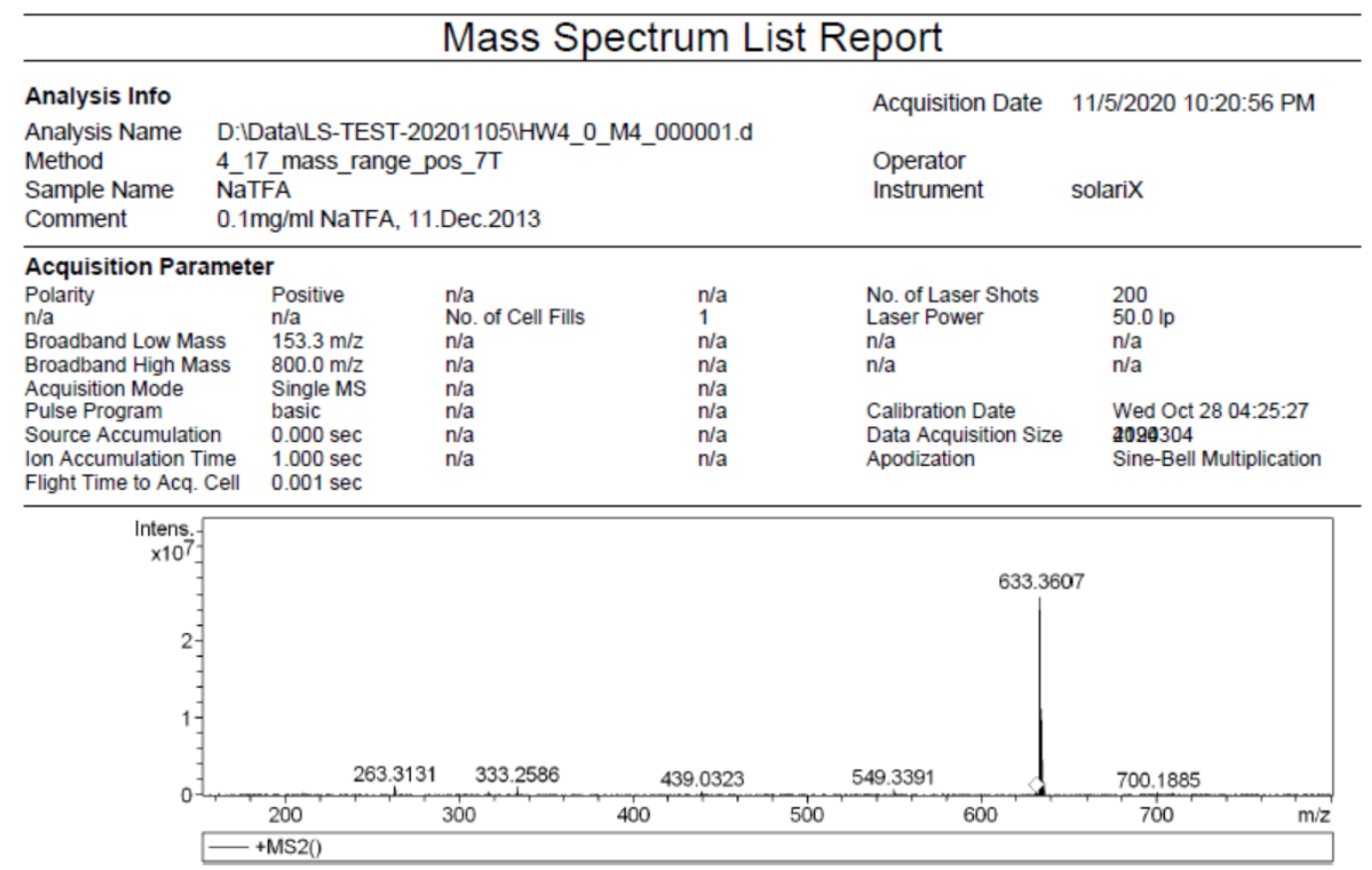

Figure $\mathbf{S}$ 10-3 HRESIMS Spectrum of $\mathbf{3 f}$ 
〈色谱图〉

$\mathrm{mV}$

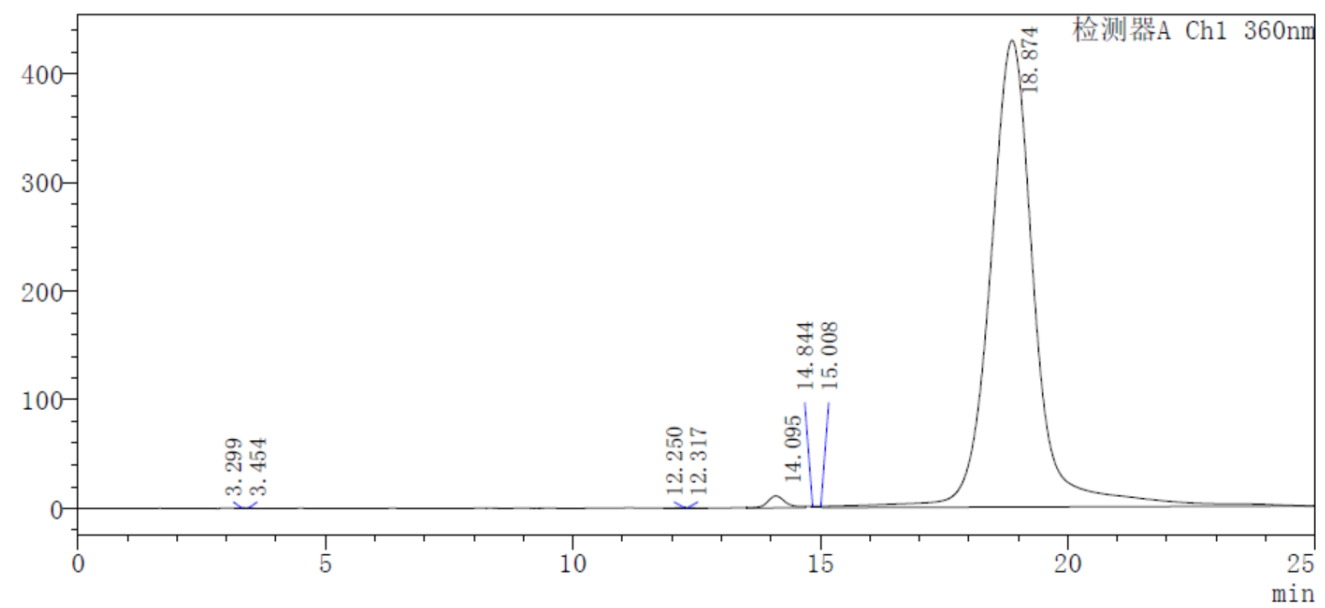

Figure S 10-4 HPLC Spectrum of $\mathbf{3 f}$

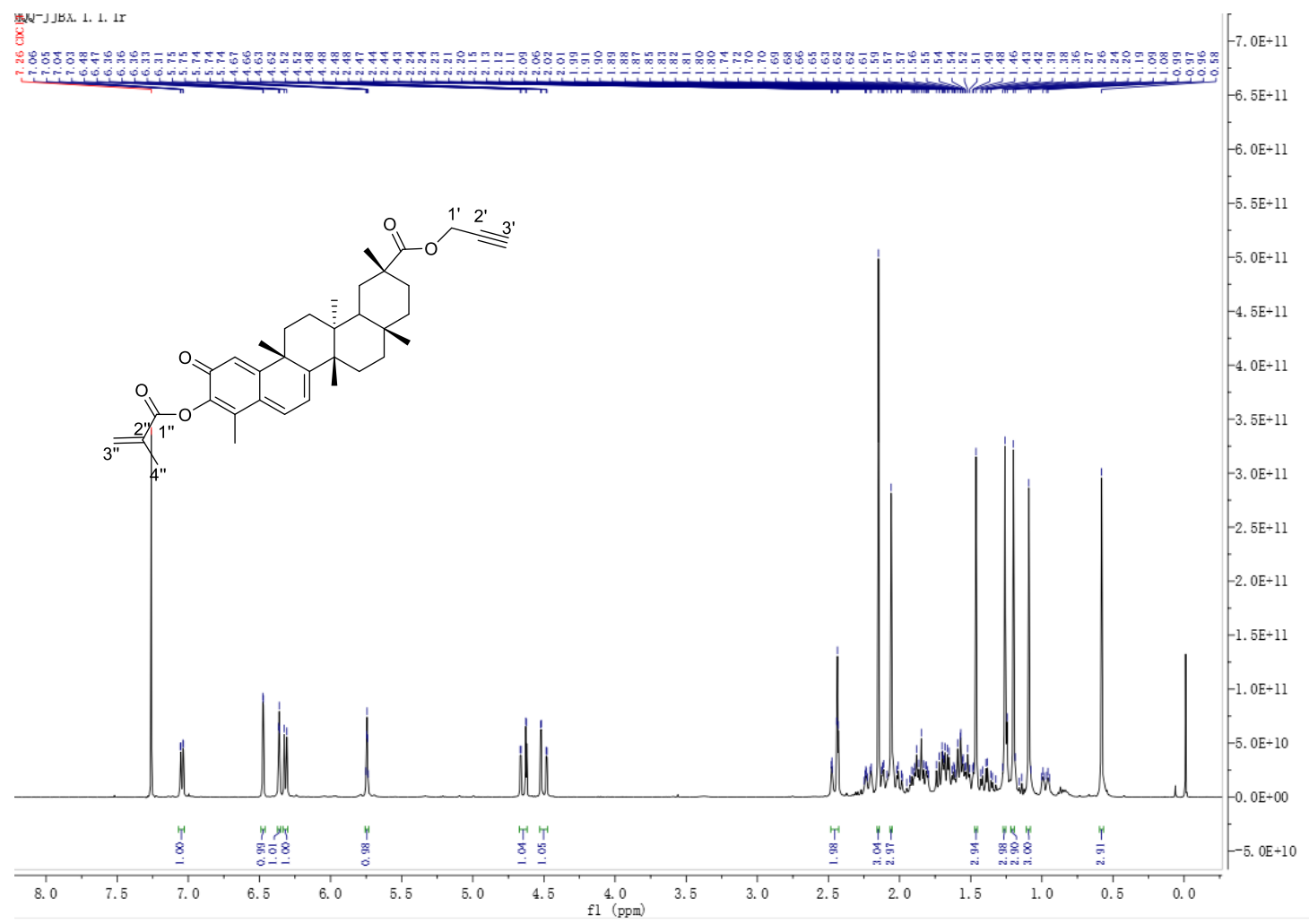

Figure $\mathbf{S}$ 11-1 ${ }^{1} \mathrm{H}$ NMR Spectrum $(400 \mathrm{MHz})$ of compound $\mathbf{3 g}$ in $\mathrm{CDCl}_{3}$ 


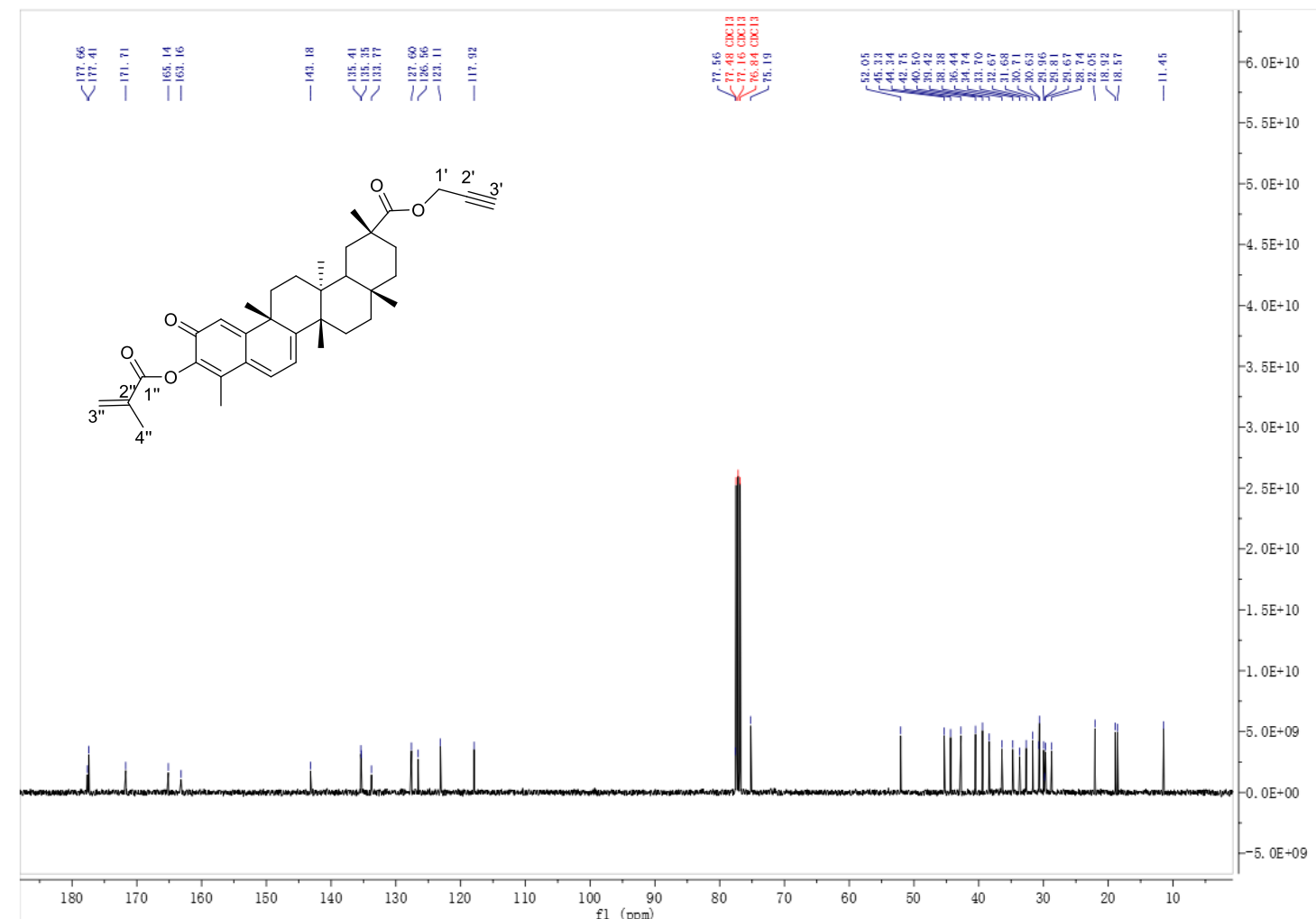

Figure S 11-2 ${ }^{13} \mathrm{C}$ NMR Spectrum $(100 \mathrm{MHz})$ of compound $\mathbf{3 g}$ in $\mathrm{CDCl}_{3}$

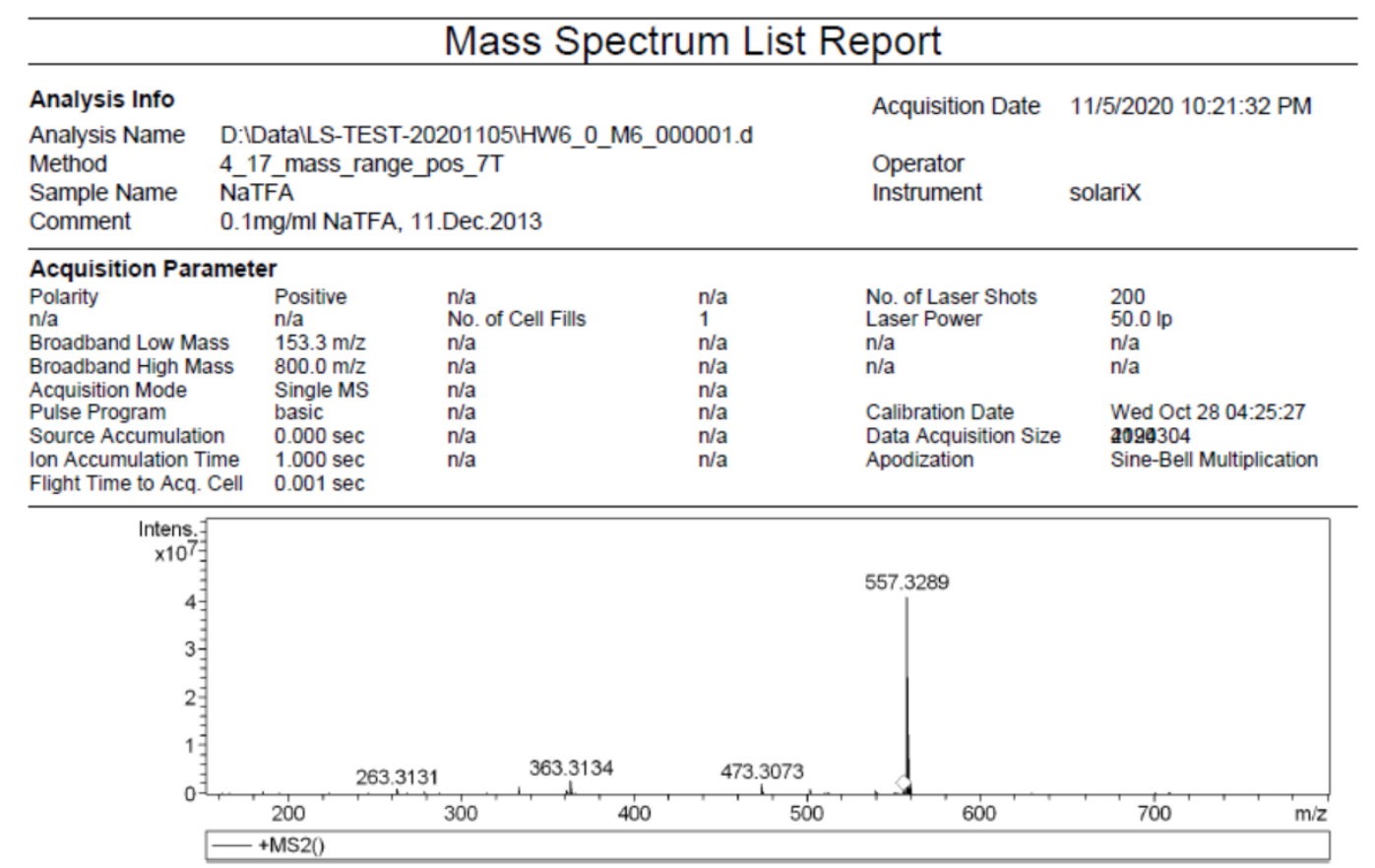

Figure S 11-3 HRESIMS Spectrum of $\mathbf{3 g}$ 
〈色谱图〉

$\mathrm{mV}$

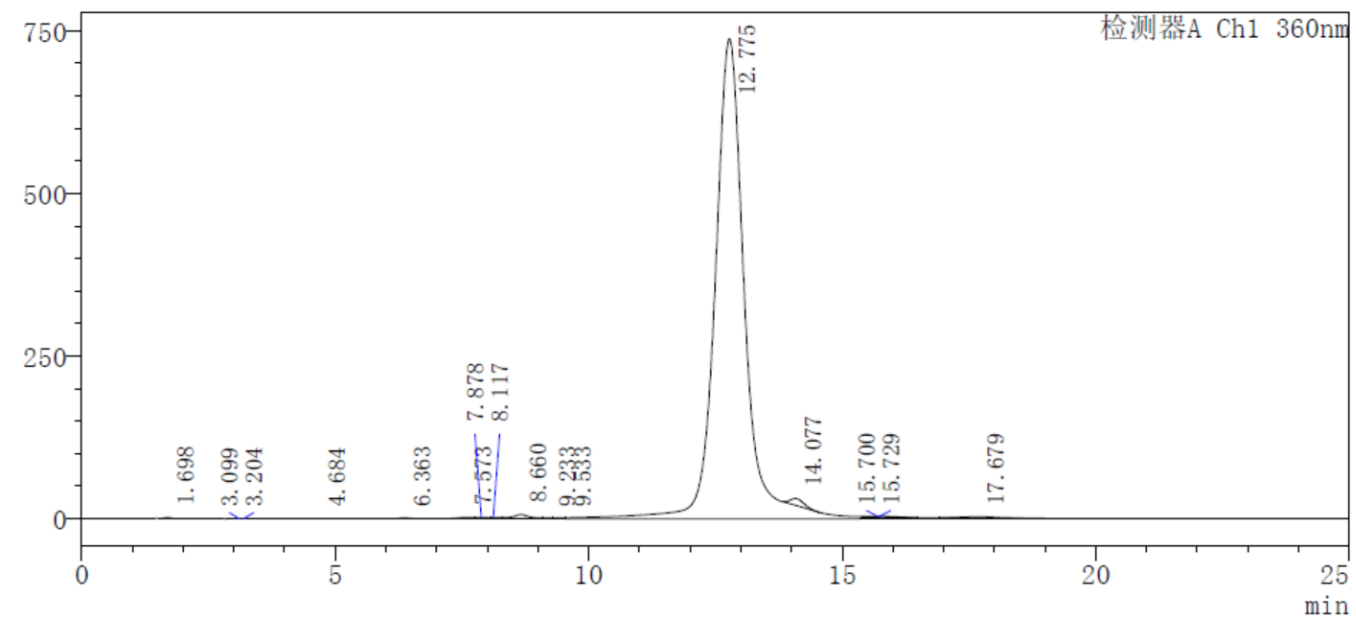

Figure S 11-4 HPLC Spectrum of 3g

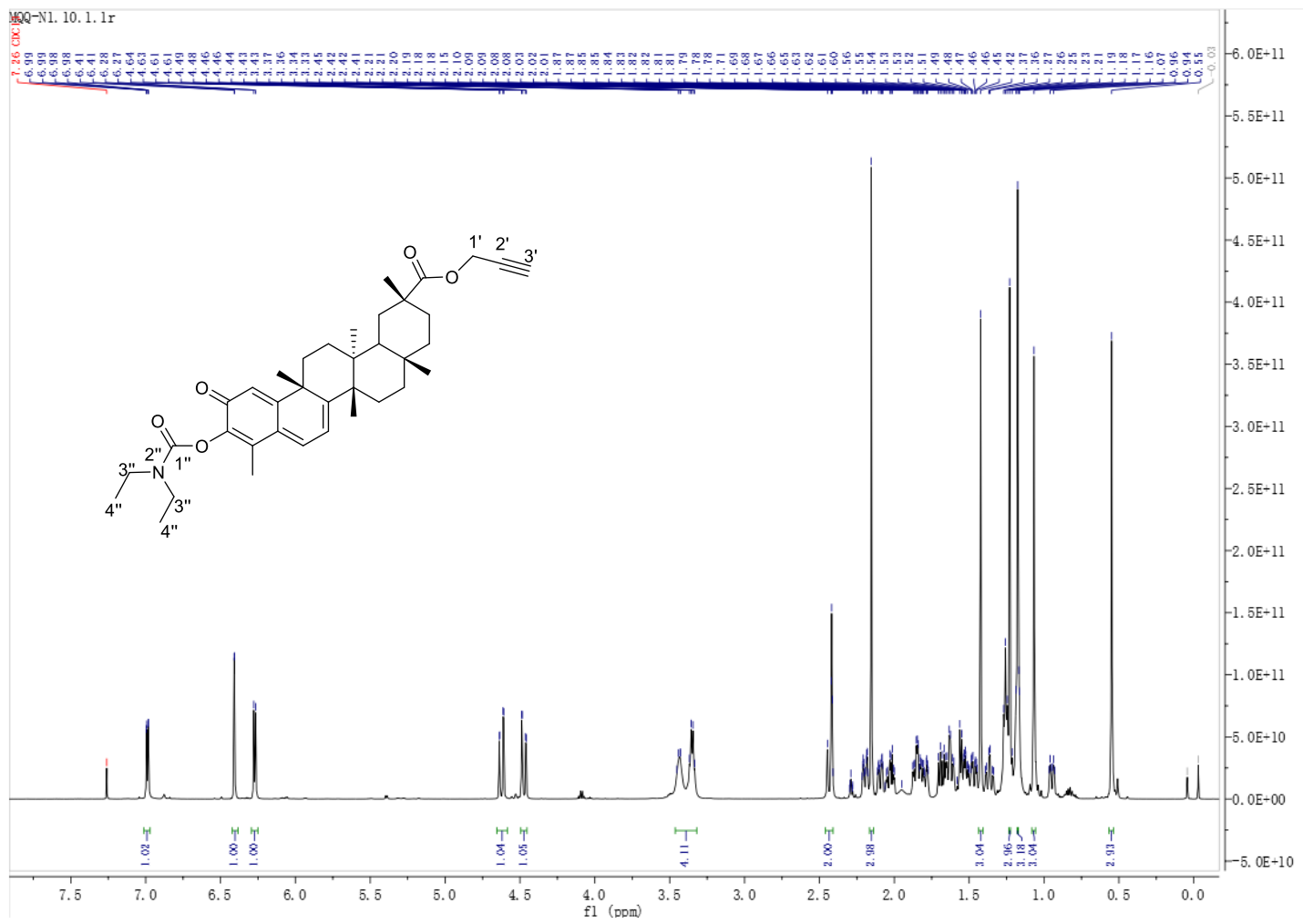

Figure $\mathbf{S}$ 12-1 ${ }^{1} \mathrm{H}$ NMR Spectrum $(600 \mathrm{MHz})$ of compound 4 a in $\mathrm{CDCl}_{3}$ 


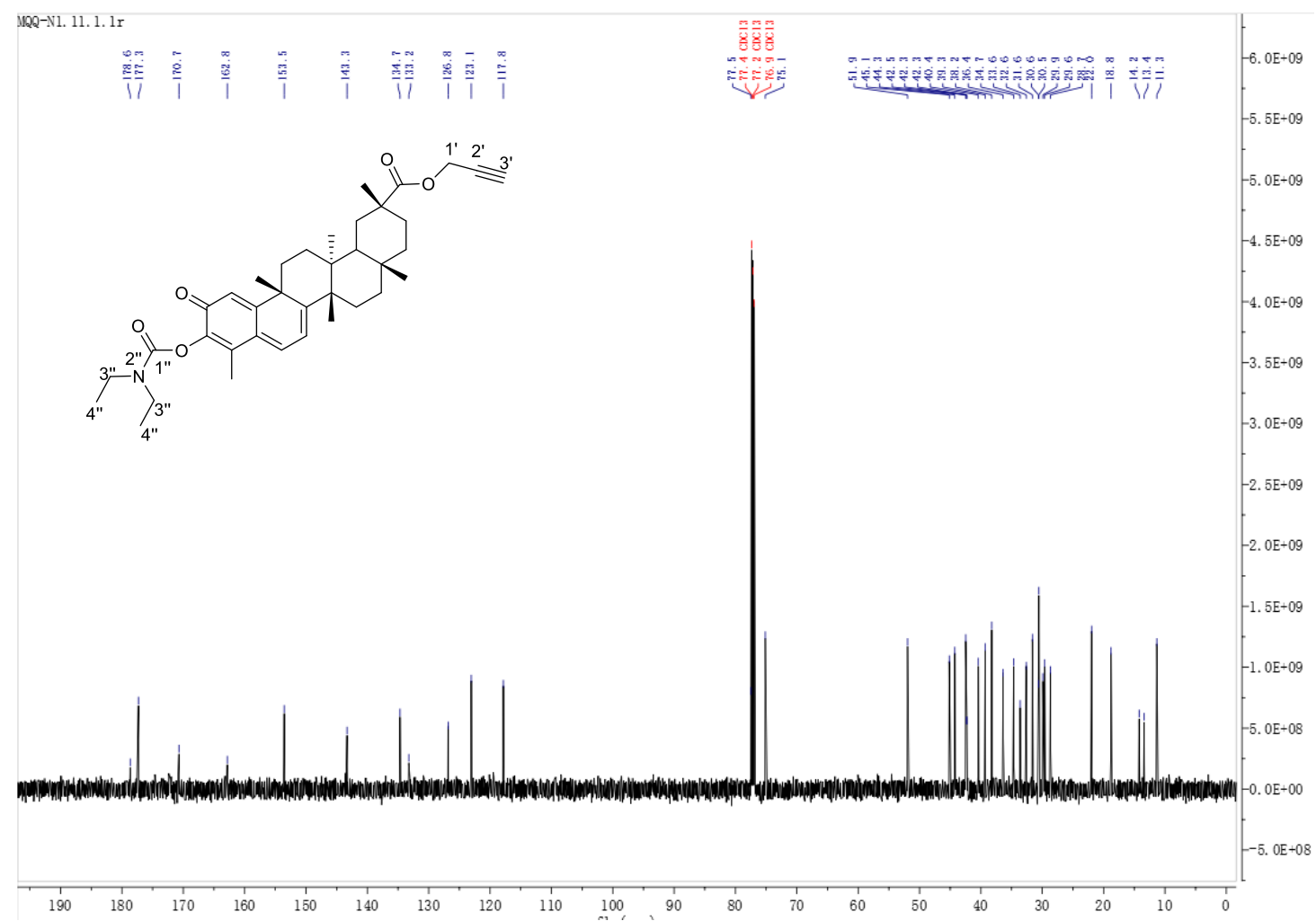

Figure S 12-2 ${ }^{13} \mathrm{C}$ NMR Spectrum $(150 \mathrm{MHz})$ of compound 4 a in $\mathrm{CDCl}_{3}$

\section{Mass Spectrum List Report}

\section{Analysis Info}

Analysis Name

Method

Sample Name

Comment

Acquisition Date $\quad 7 / 11 / 2020$ 6:13:55 PM

D:IDatalLS-TEST20200711 ICE-N1_0_L1_000001.d

4_17_mass_range_pos_7T

NaTFA

$0.1 \mathrm{mg} / \mathrm{ml}$ NaTFA, 11.Dec. 2013

\section{Acquisition Parameter}

Polarity
n/a

Broadband Low Mass $92.0 \mathrm{~m} / \mathrm{z}$

Broadband High Mass $\quad 1600.0 \mathrm{~m} / \mathrm{z}$

Acquisition Mode

Pulse Program

Ion Accumulation Time

Single MS

basic

0.000 sec

$0.000 \mathrm{sec}$

$0.015 \mathrm{sec}$

$0.001 \mathrm{sec}$

n/a
No. of Cell Fills
n/a
n/a
n/a
n/a
n/a
n/a

$n / a$
1
$n / a$
$n / a$
$n / a$
$n / a$
$n / a$
$n / a$

No. of Laser Shots

Laser Power

$n / a$

Operator

Instrument solariX

Flight Time to Acq. Cell

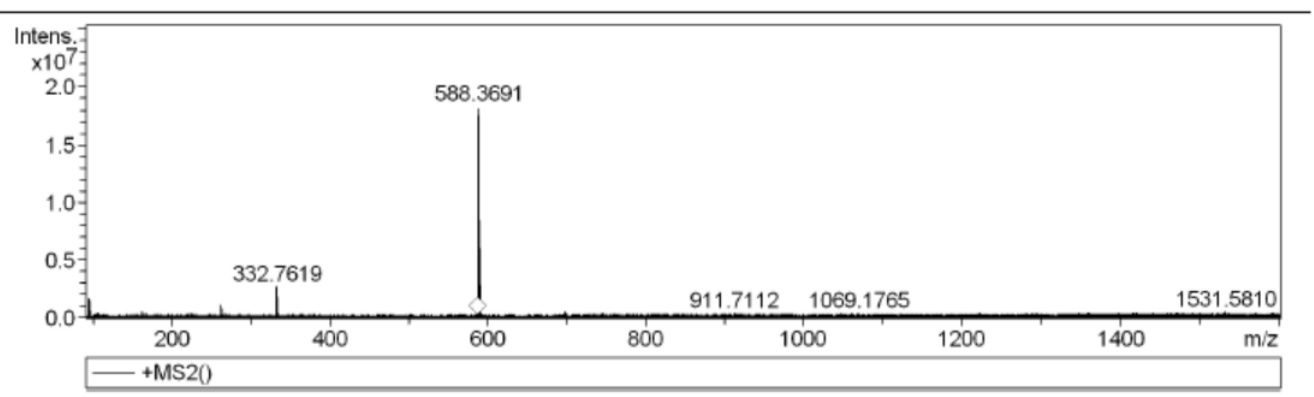

Figure S 12-3 HRESIMS Spectrum of 4a 
〈色谱图〉

$\mathrm{mV}$

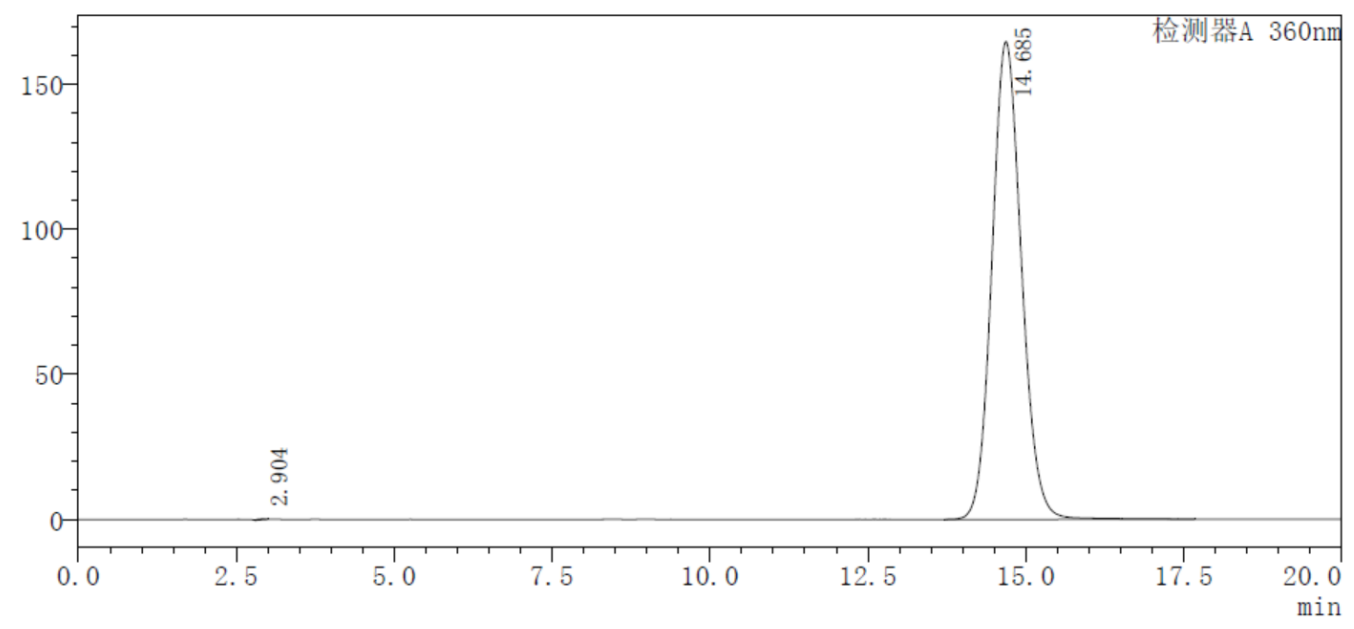

Figure S 12-4 HPLC Spectrum of $\mathbf{4 a}$

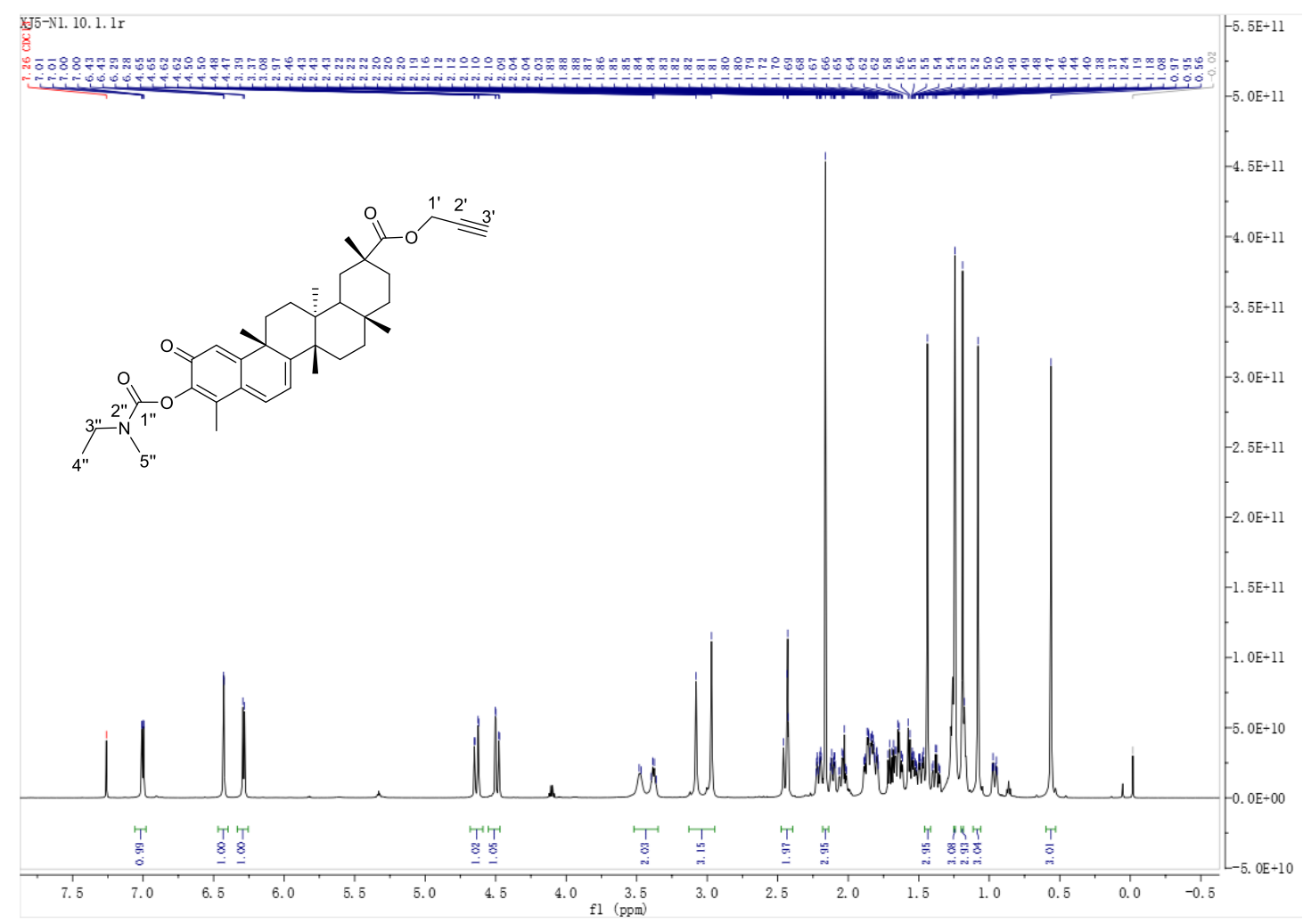

Figure S 13-1 ${ }^{1} \mathrm{H}$ NMR Spectrum (600MHz) of compound $\mathbf{4 b}$ in $\mathrm{CDCl}_{3}$ 


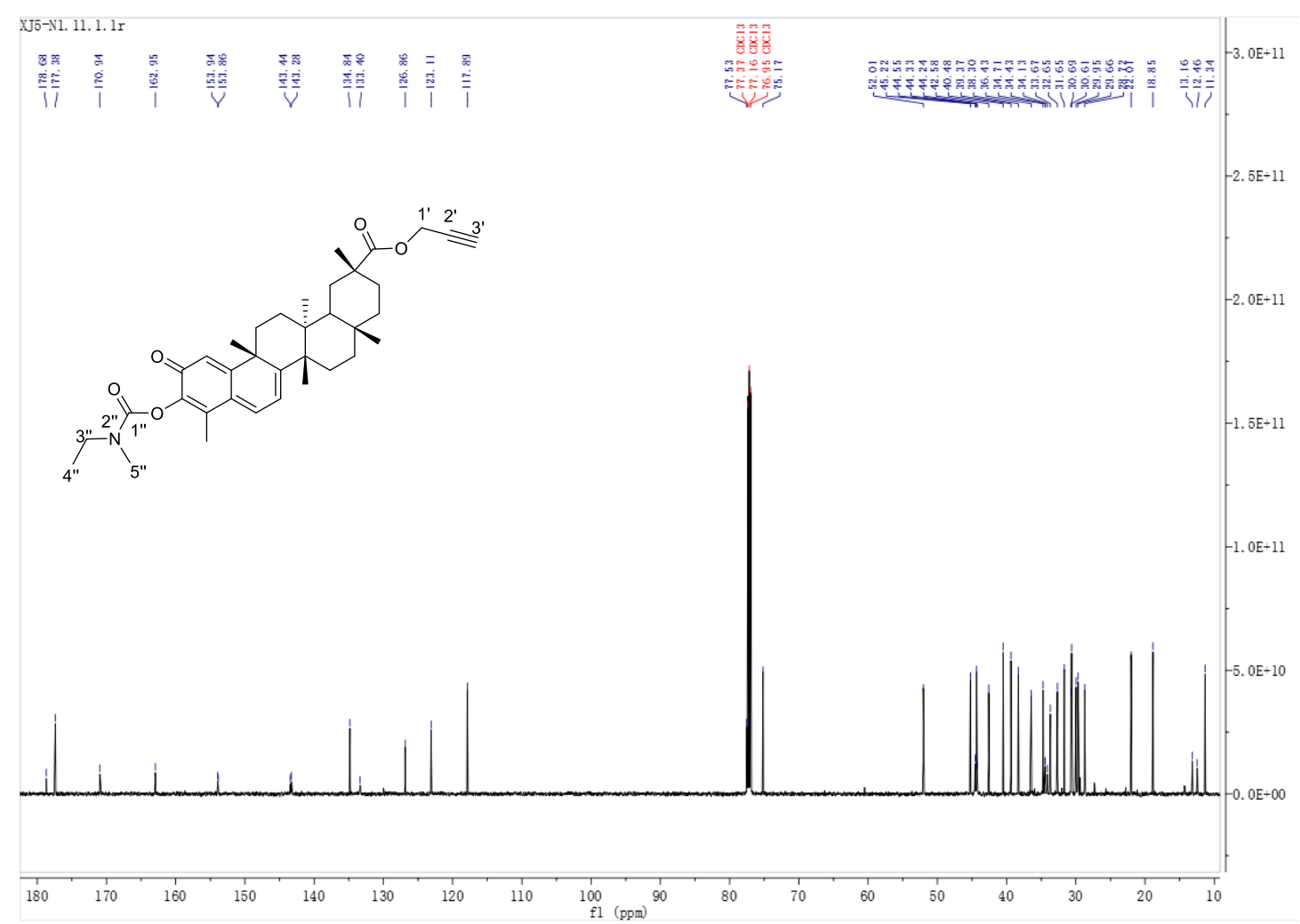

Figure S 13-2 ${ }^{13} \mathrm{C}$ NMR Spectrum $(150 \mathrm{MHz})$ of compound $\mathbf{4 b}$ in $\mathrm{CDCl}_{3}$

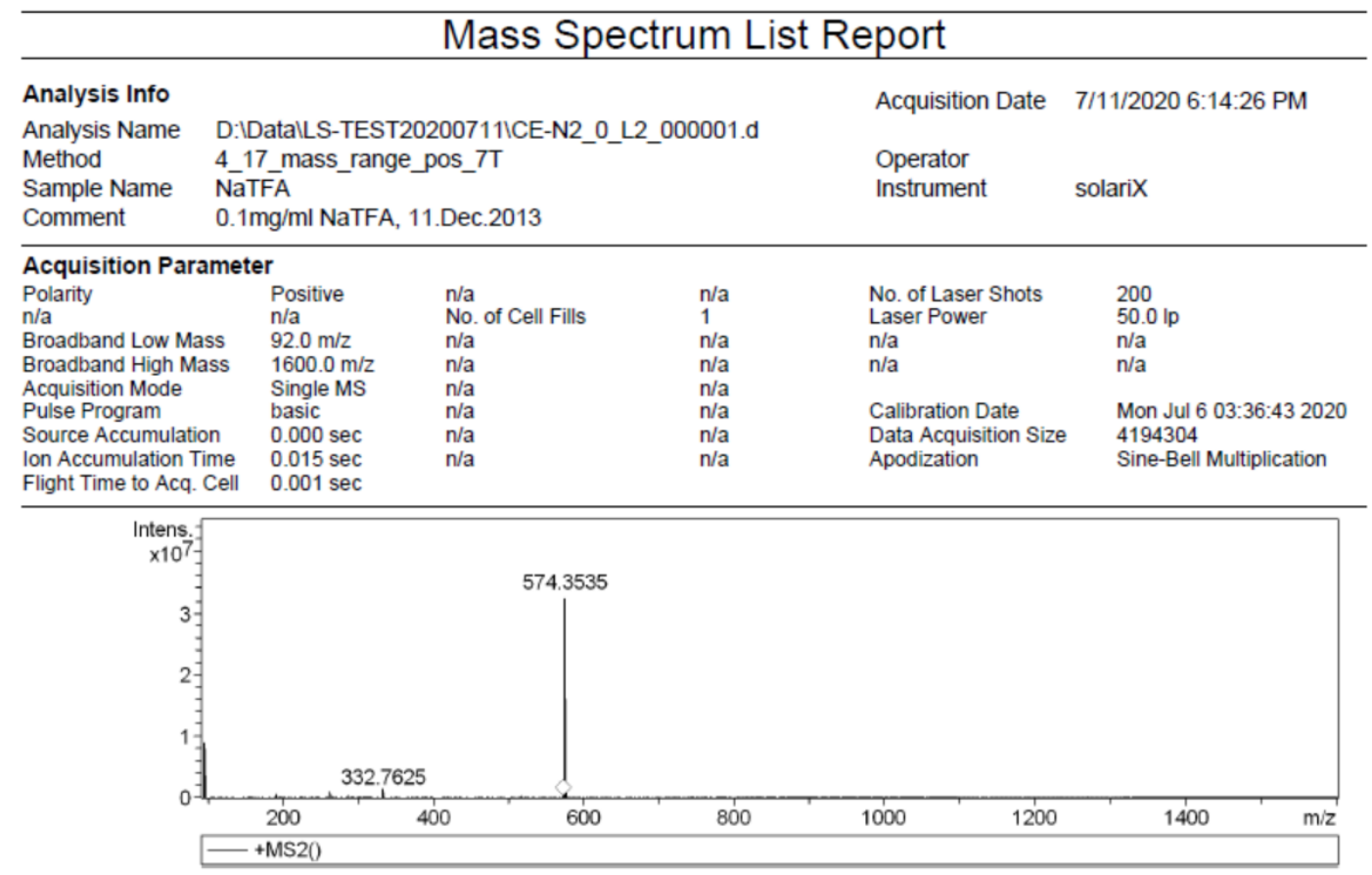

Figure S 13-3 HRESIMS Spectrum of $4 b$ 
〈色谱图〉

$\mathrm{mV}$

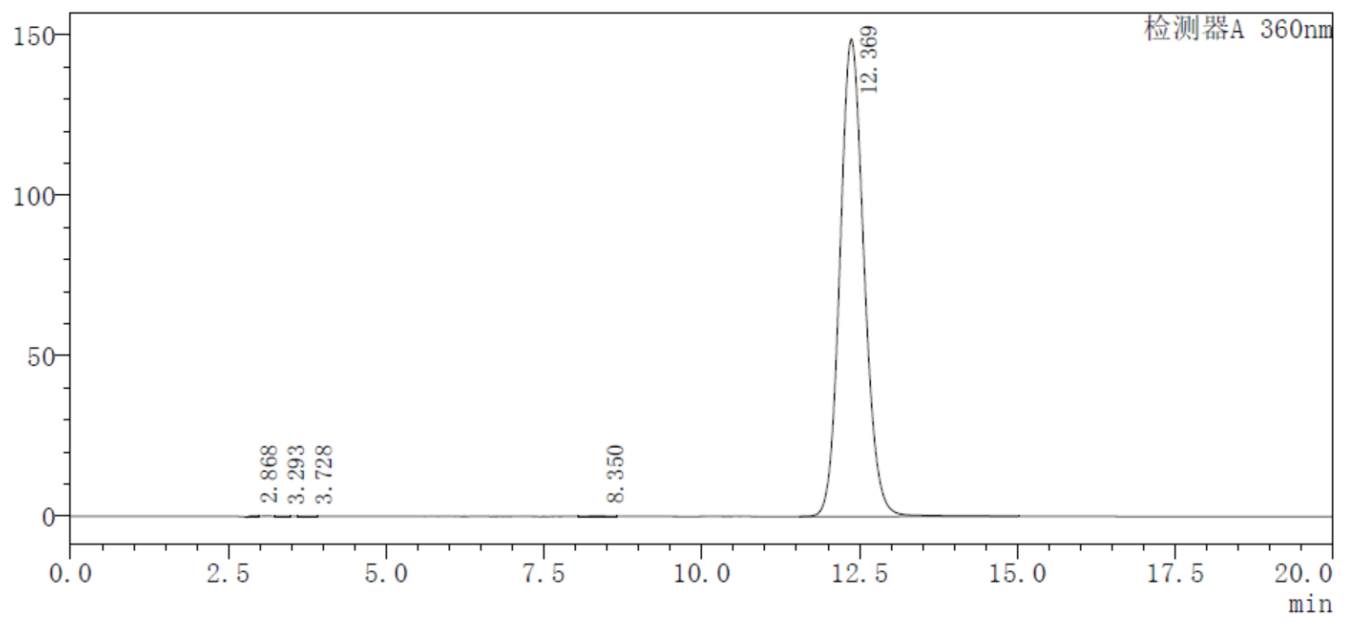

Figure S 13-4 HPLC Spectrum of 4b

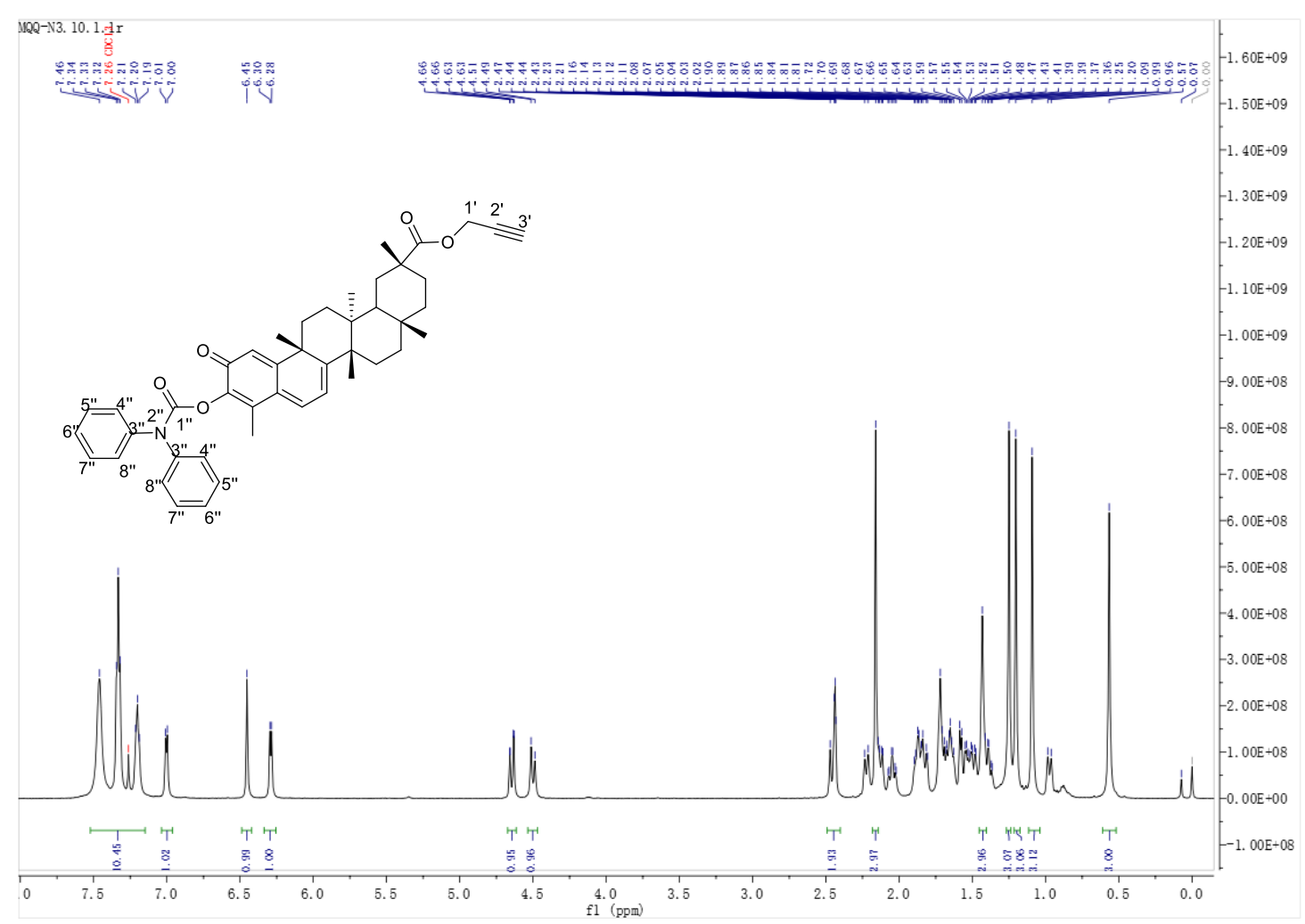

Figure S 14-1 ${ }^{1} \mathrm{H}$ NMR Spectrum $(600 \mathrm{MHz})$ of compound $4 \mathbf{c}$ in $\mathrm{CDCl}_{3}$ 


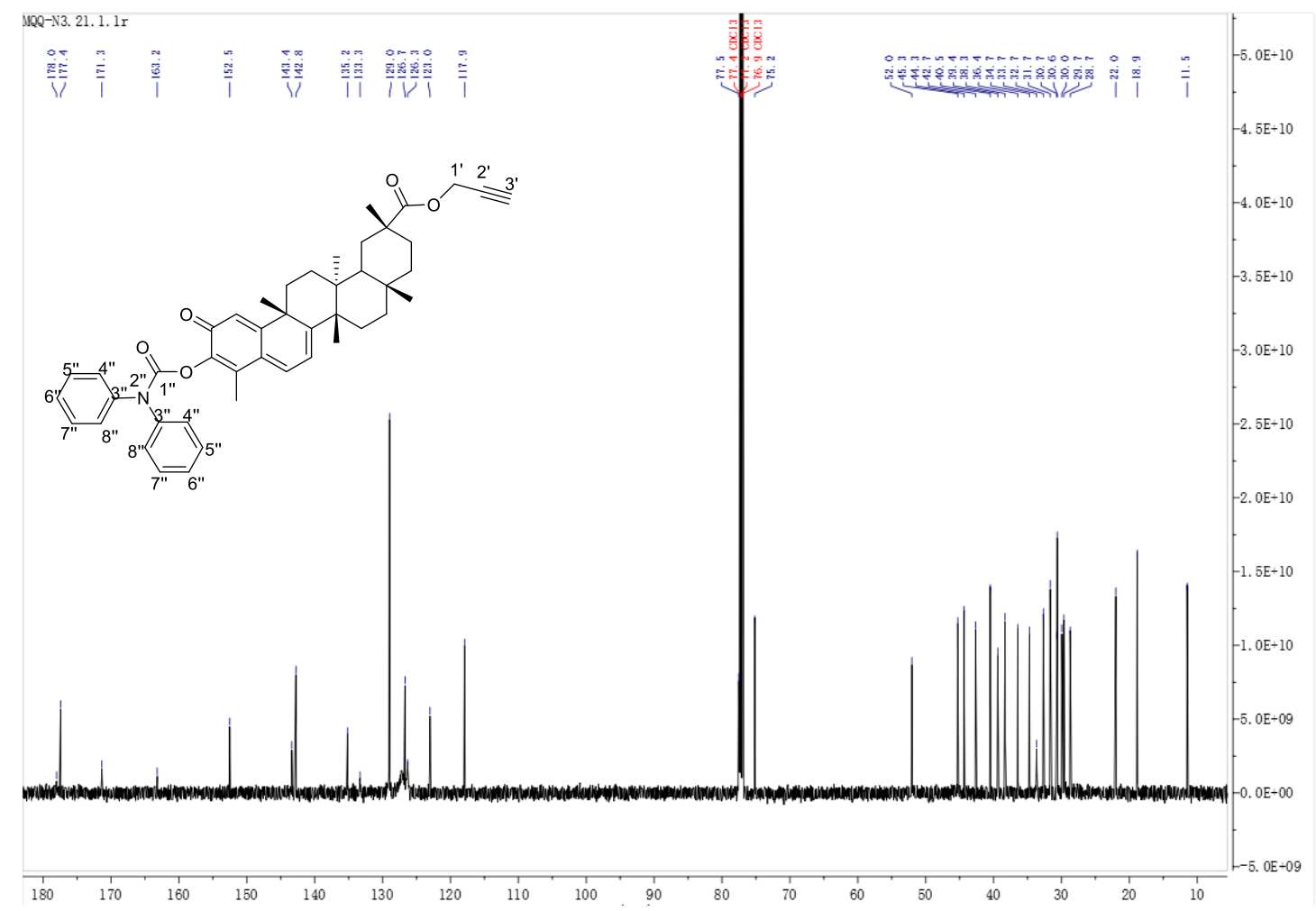

Figure S 14-2 ${ }^{13} \mathrm{C}$ NMR Spectrum $(150 \mathrm{MHz})$ of compound $4 \mathbf{c}$ in $\mathrm{CDCl}_{3}$

\section{Mass Spectrum List Report}

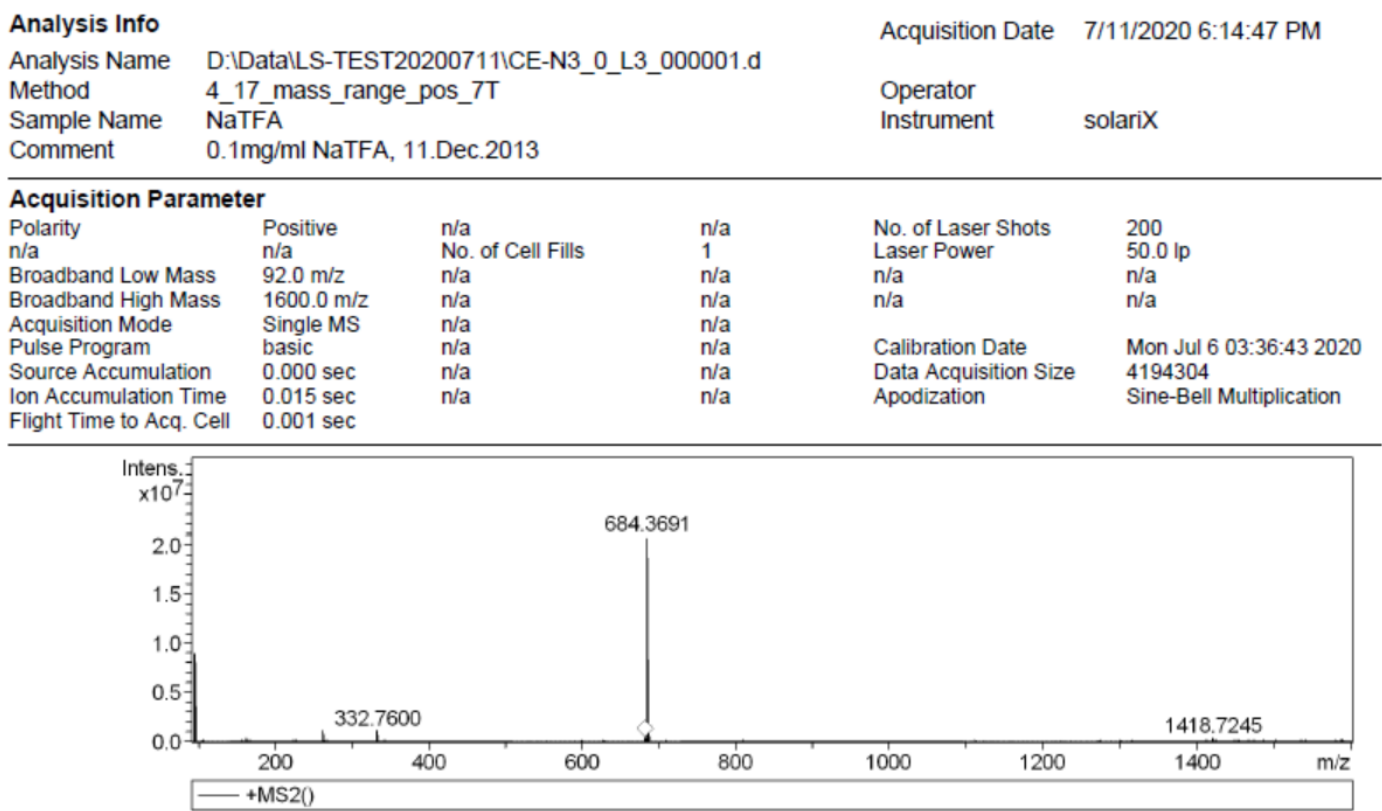

Figure S 14-3 HRESIMS Spectrum of 4c 
〈色谱图〉

$\mathrm{mV}$

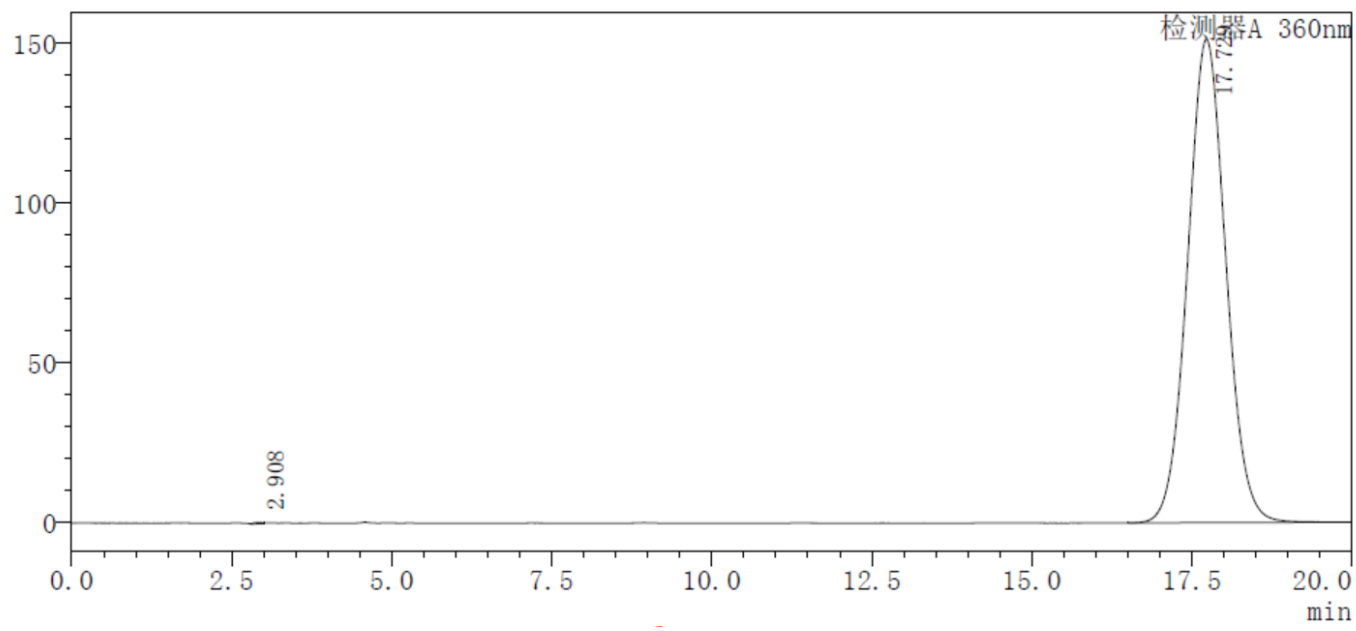

Figure S 14-4 HPLC Spectrum of 4c

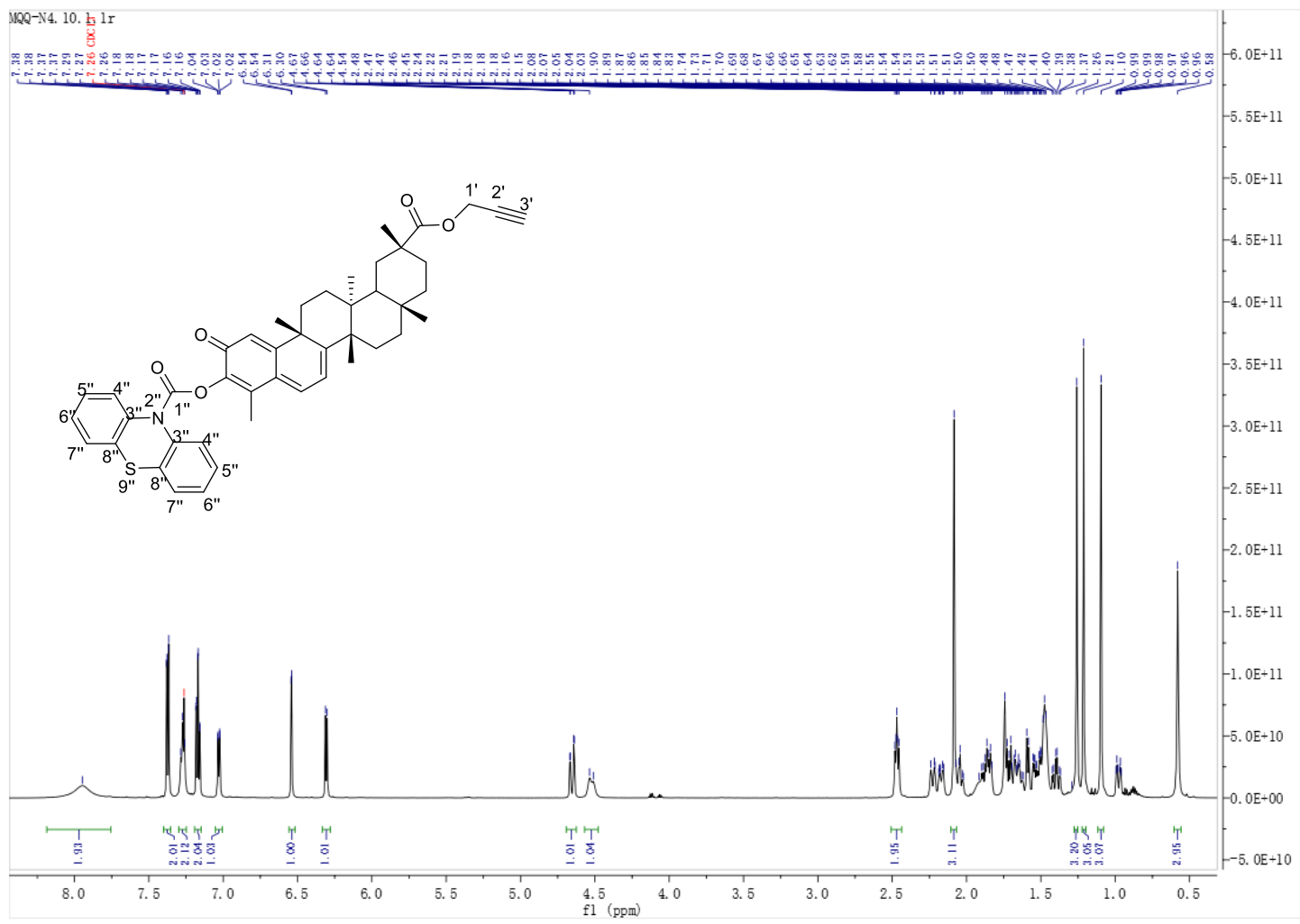

Figure $\mathbf{S}$ 15-1 ${ }^{1} \mathrm{H}$ NMR Spectrum $(600 \mathrm{MHz})$ of compound $\mathbf{4 d}$ in $\mathrm{CDCl}_{3}$ 


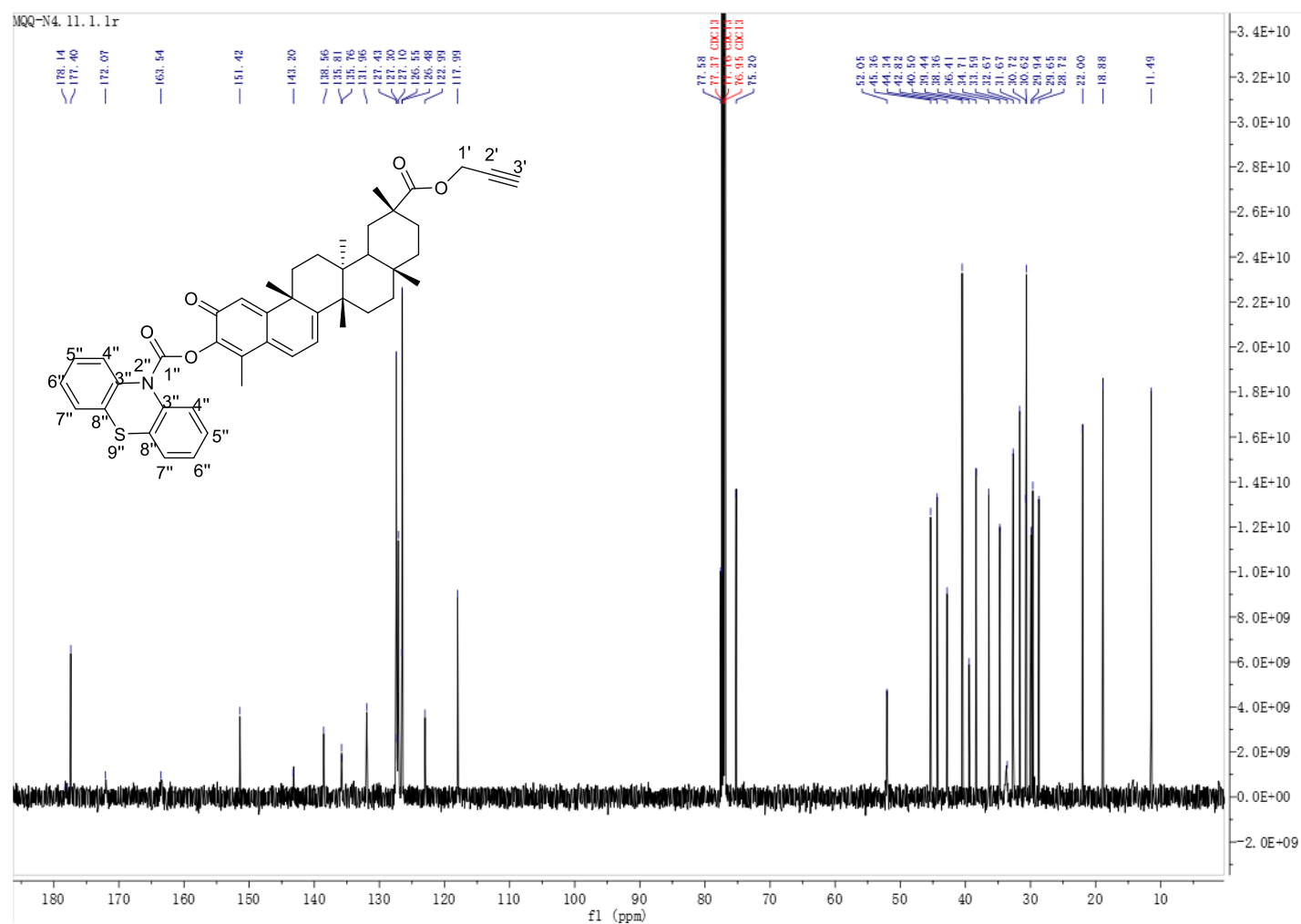

Figure $\mathbf{S}$ 15-2 ${ }^{13} \mathrm{C}$ NMR Spectrum $(150 \mathrm{MHz})$ of compound $4 \mathbf{d}$ in $\mathrm{CDCl}_{3}$

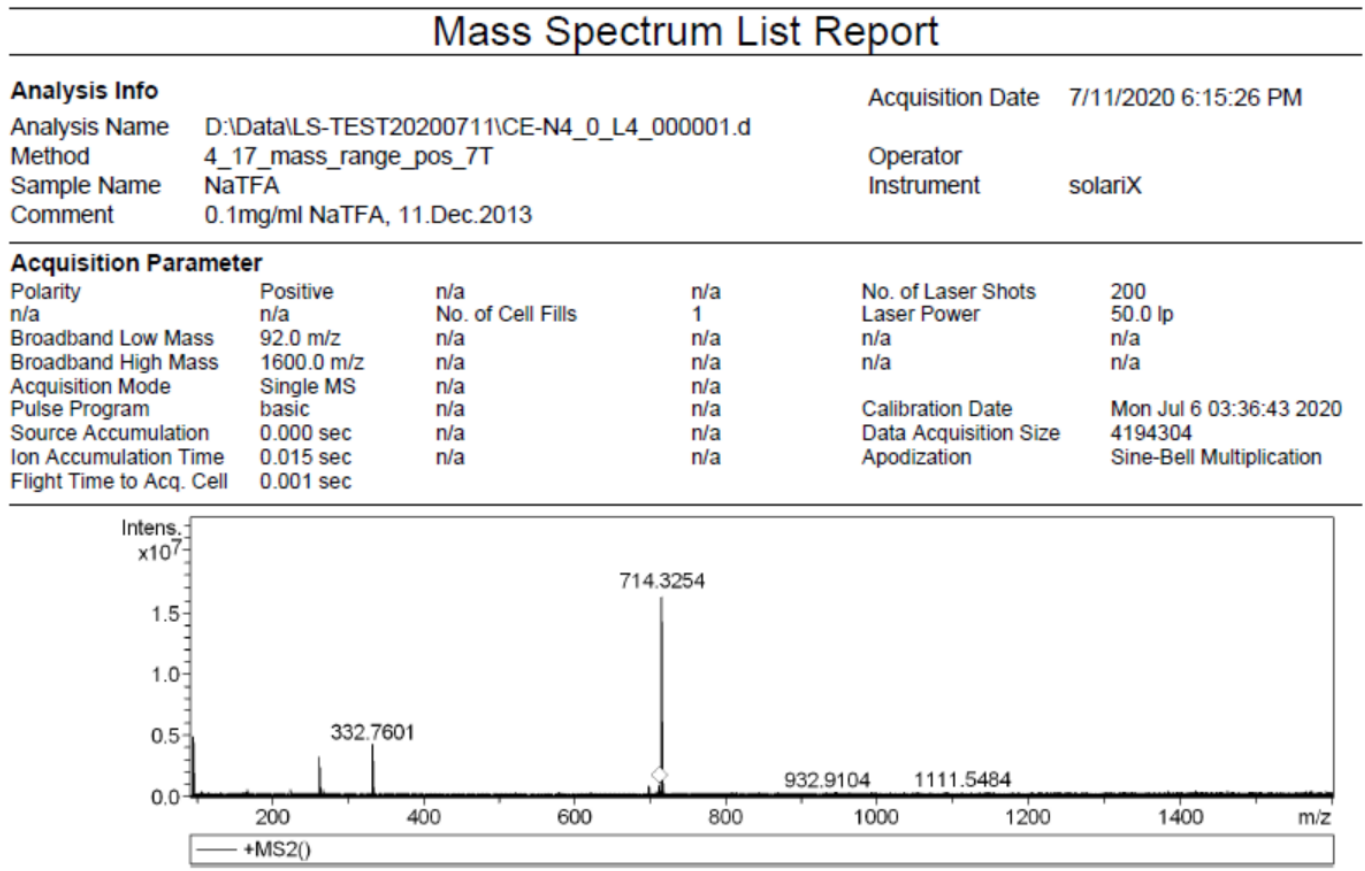

Figure S 15-3 HRESIMS Spectrum of 4d 
〈色谱图〉

$\mathrm{mV}$

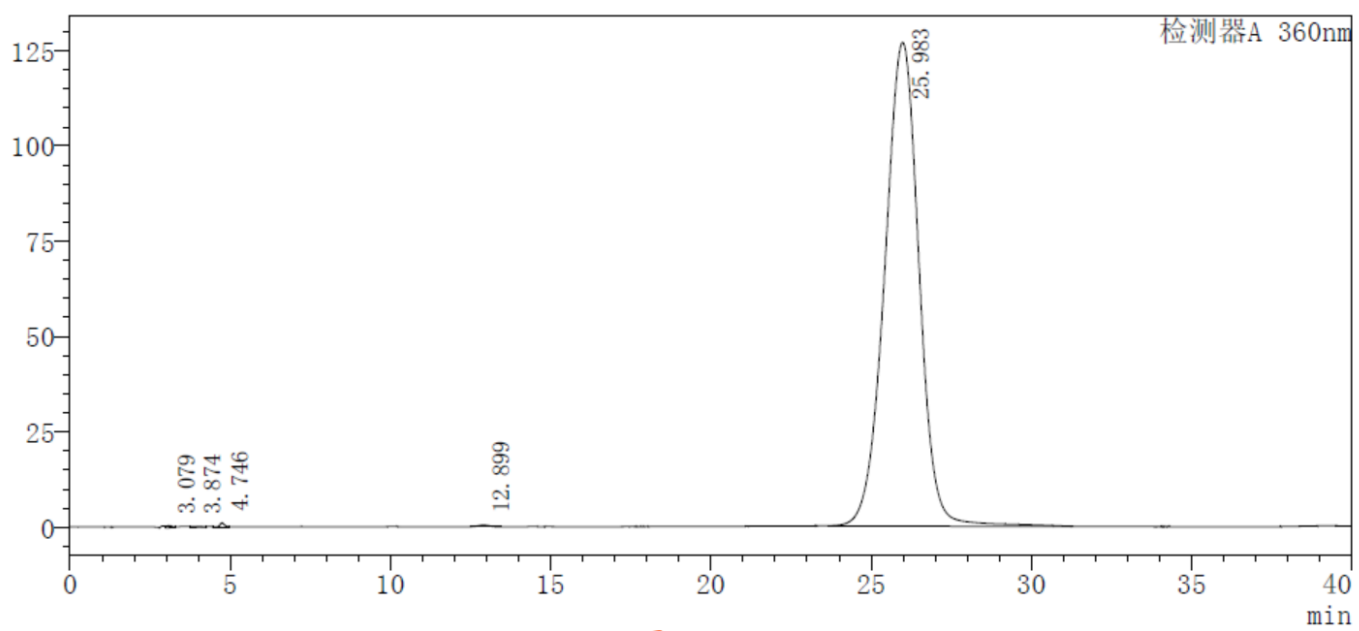

Figure S 15-4 HPLC Spectrum of 4d

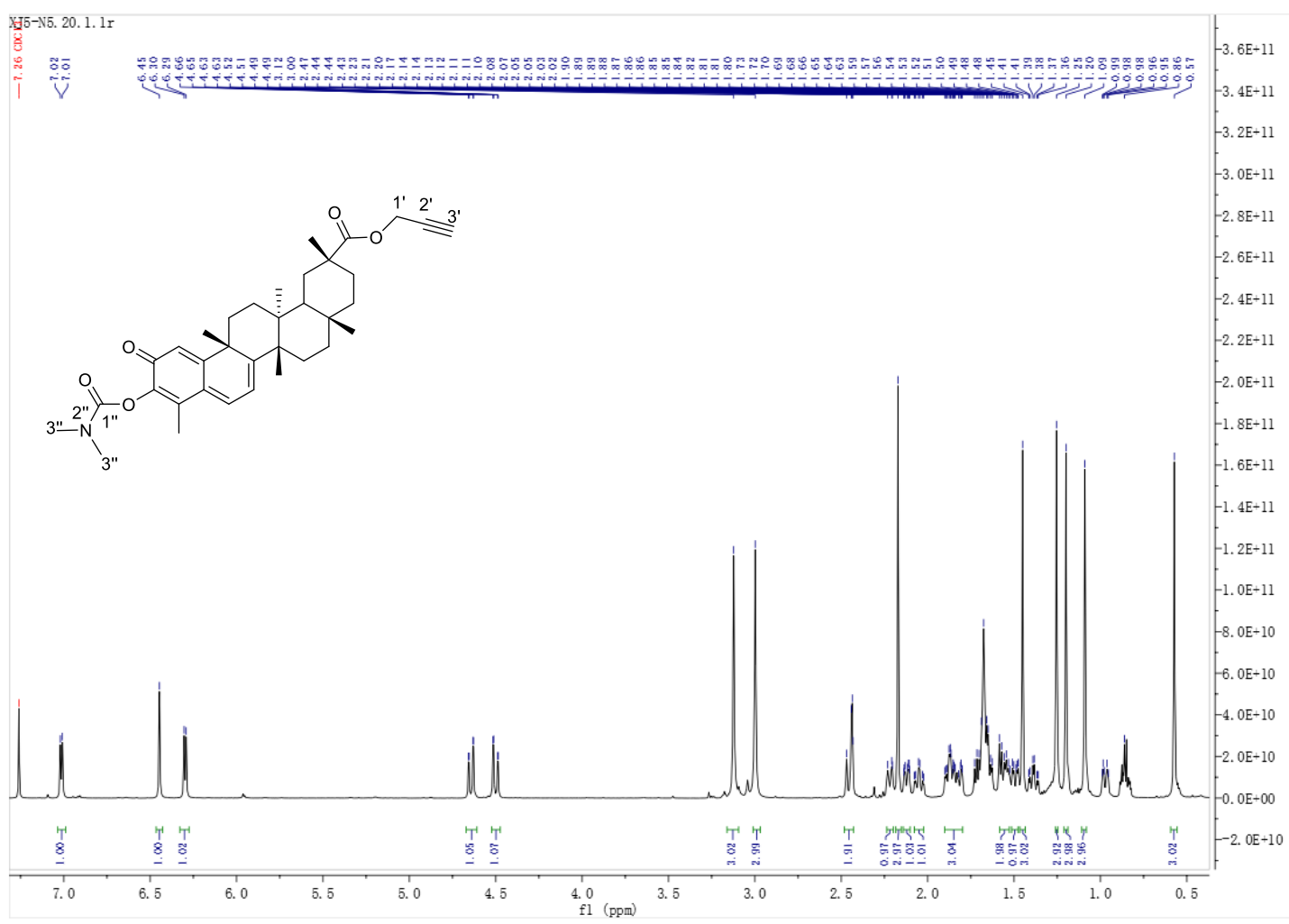

Figure $\mathbf{S}$ 16-1 ${ }^{1} \mathrm{H}$ NMR Spectrum $(600 \mathrm{MHz})$ of compound $4 \mathbf{e}$ in $\mathrm{CDCl}_{3}$ 


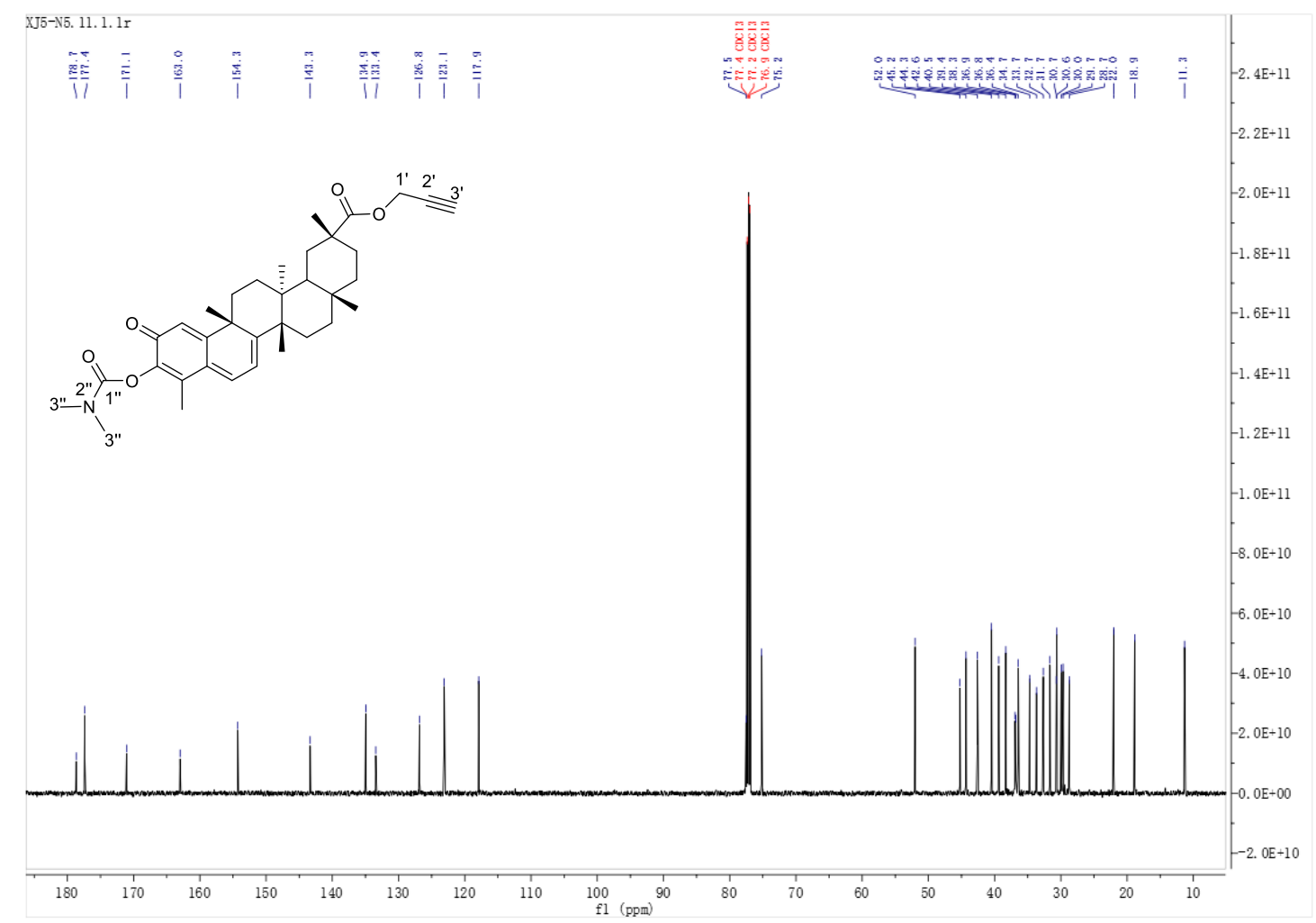

Figure S 16-2 ${ }^{13} \mathrm{C}$ NMR Spectrum $(150 \mathrm{MHz})$ of compound $4 \mathbf{e}$ in $\mathrm{CDCl}_{3}$

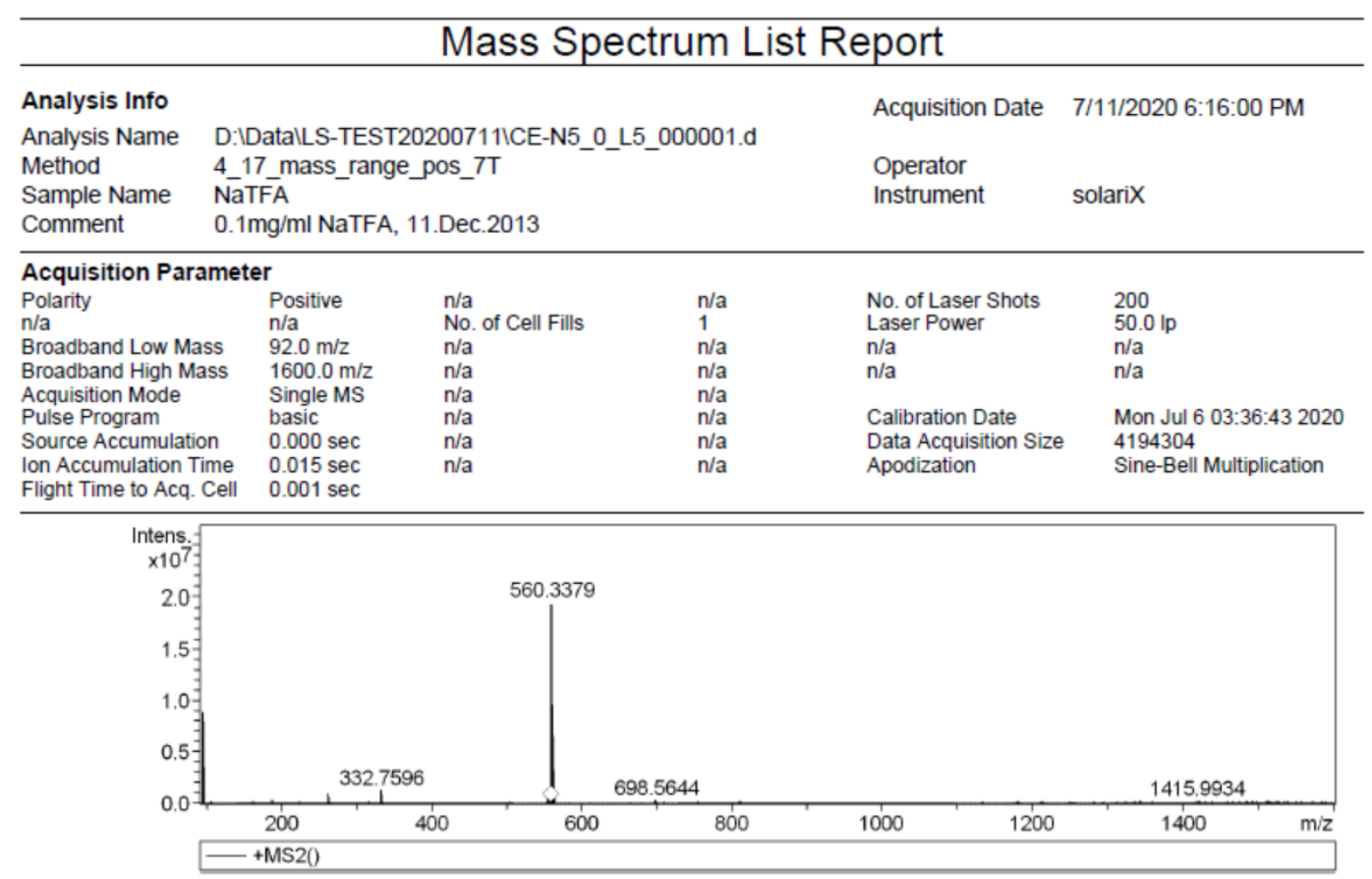

Figure S 16-3 HRESIMS Spectrum of $4 \mathrm{e}$ 
〈色谱图〉

$\mathrm{mV}$

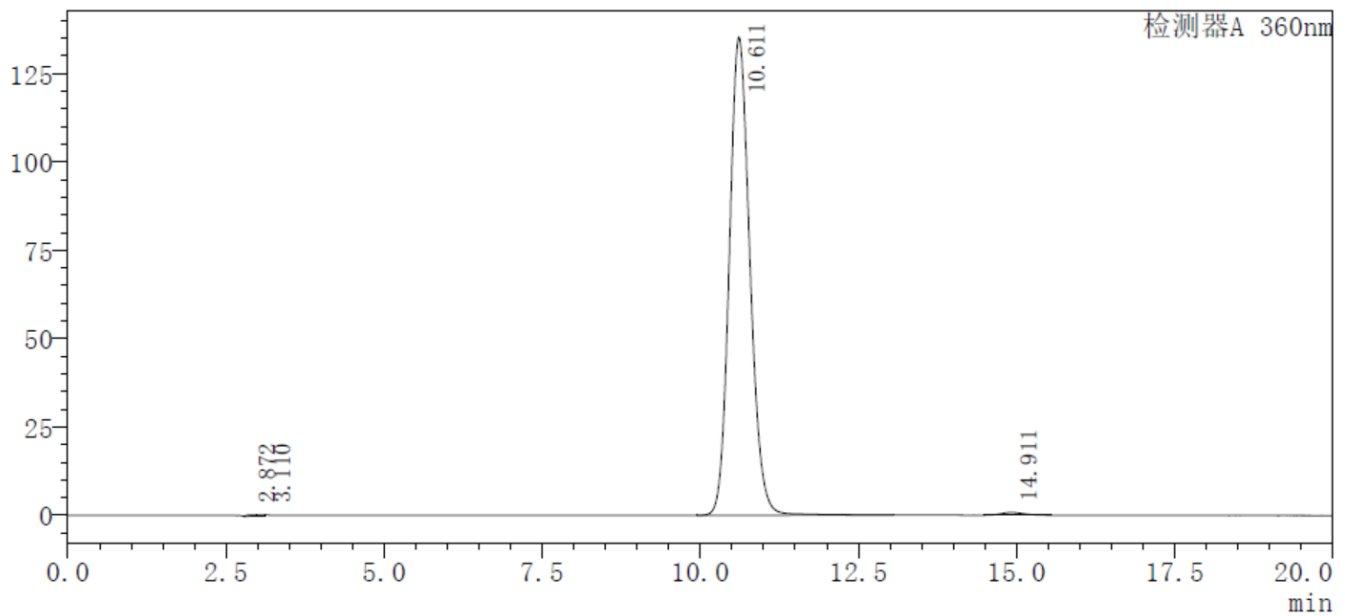

Figure S 16-4 HPLC Spectrum of 4e

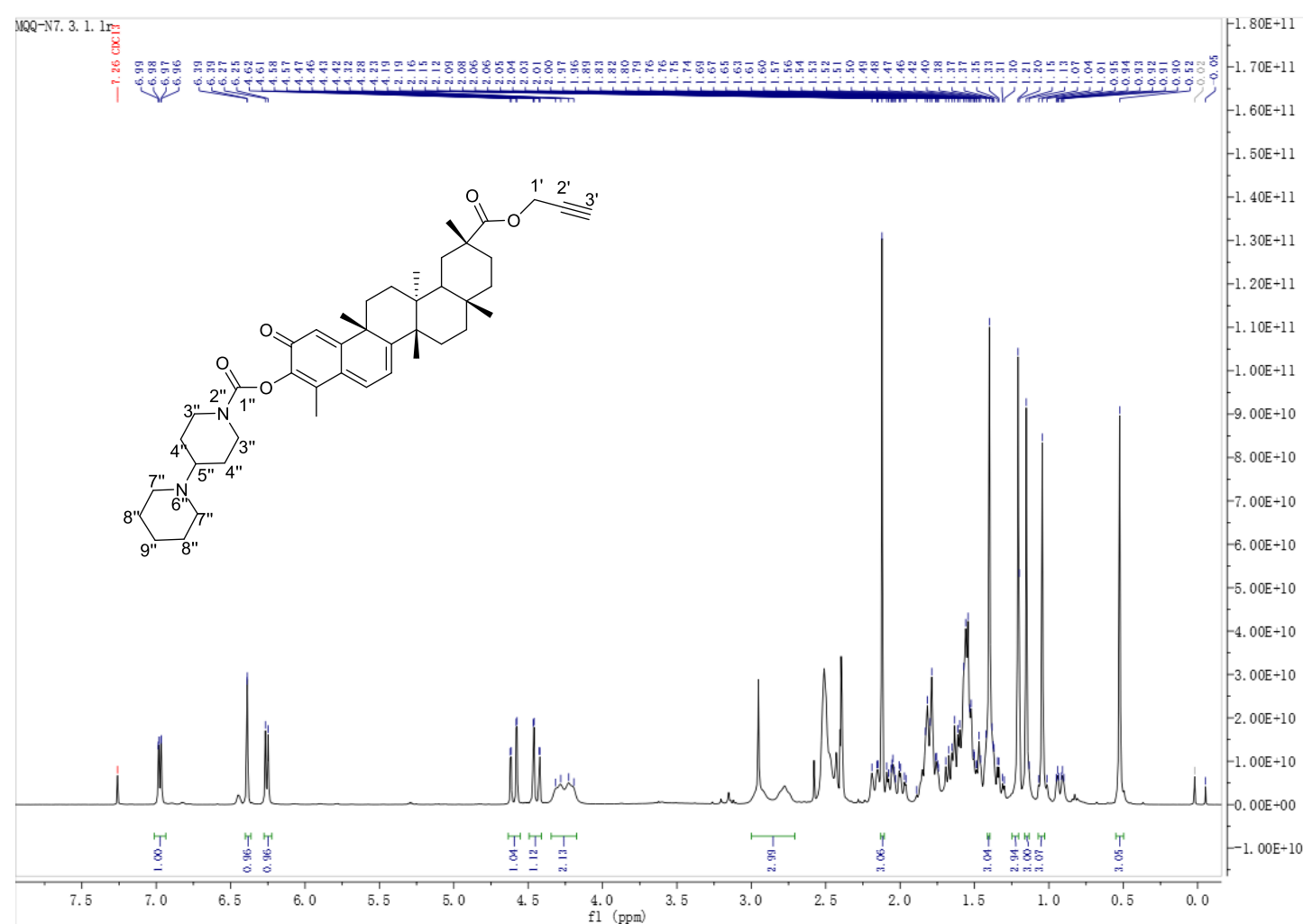

Figure $\mathbf{S}$ 17-1 ${ }^{1} \mathrm{H}$ NMR Spectrum $(600 \mathrm{MHz})$ of compound $\mathbf{4 f}$ in $\mathrm{CDCl}_{3}$ 


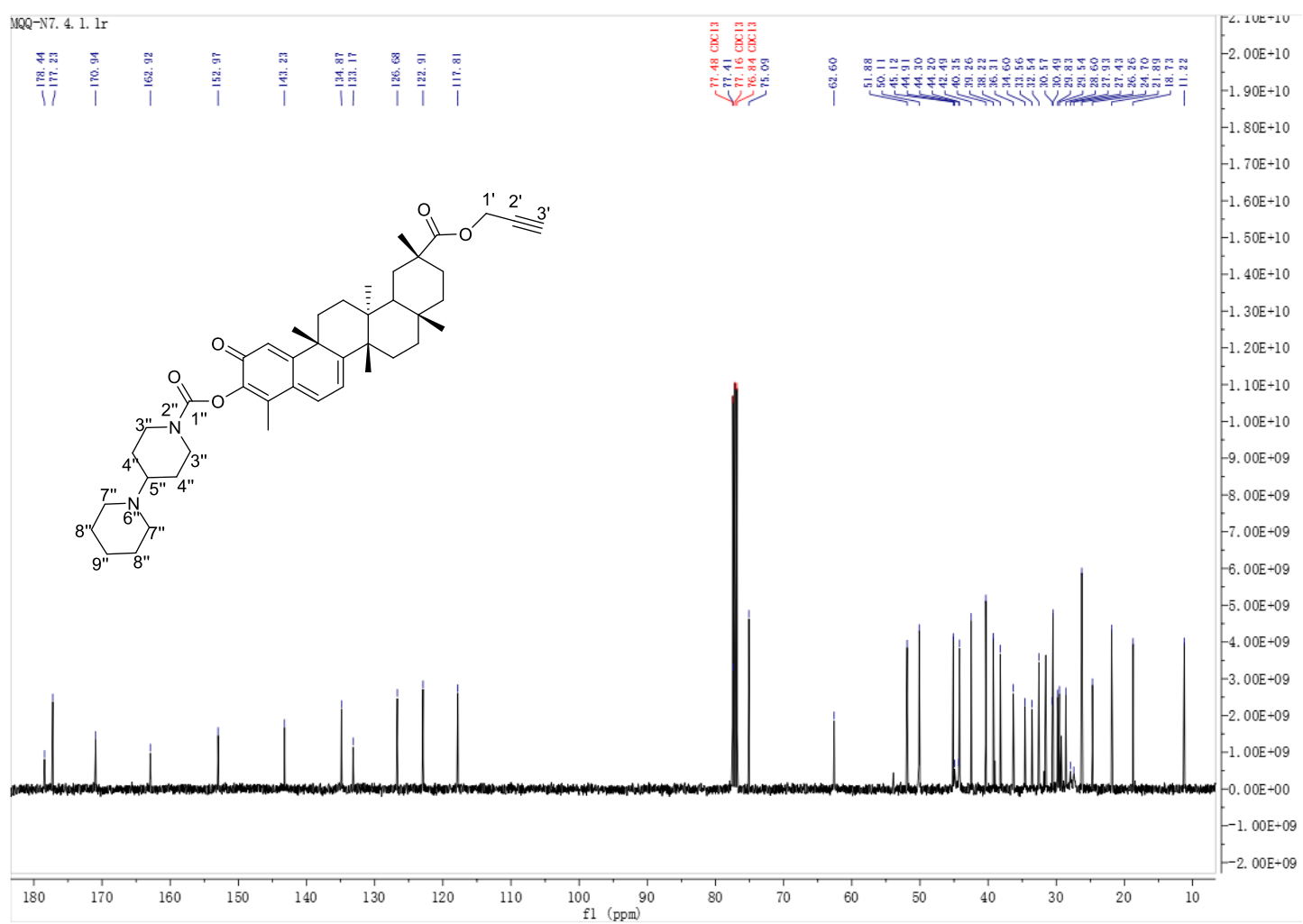

Figure S 17-2 ${ }^{13} \mathrm{C}$ NMR Spectrum $(150 \mathrm{MHz})$ of compound $\mathbf{4 f}$ in $\mathrm{CDCl}_{3}$

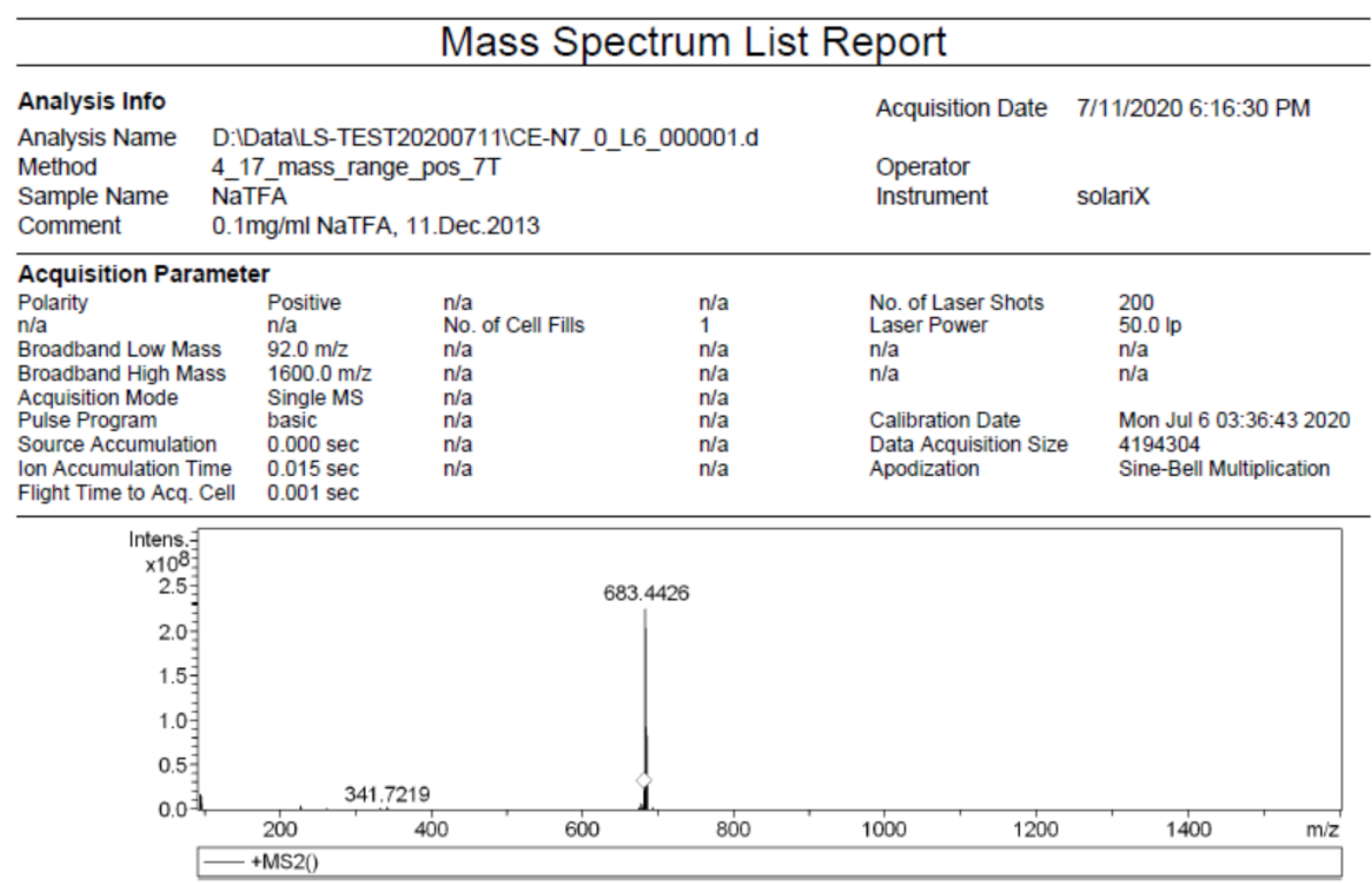

Figure $\mathbf{S}$ 17-3 HRESIMS Spectrum of $\mathbf{4 f}$ 
〈色谱图〉

$\mathrm{mV}$

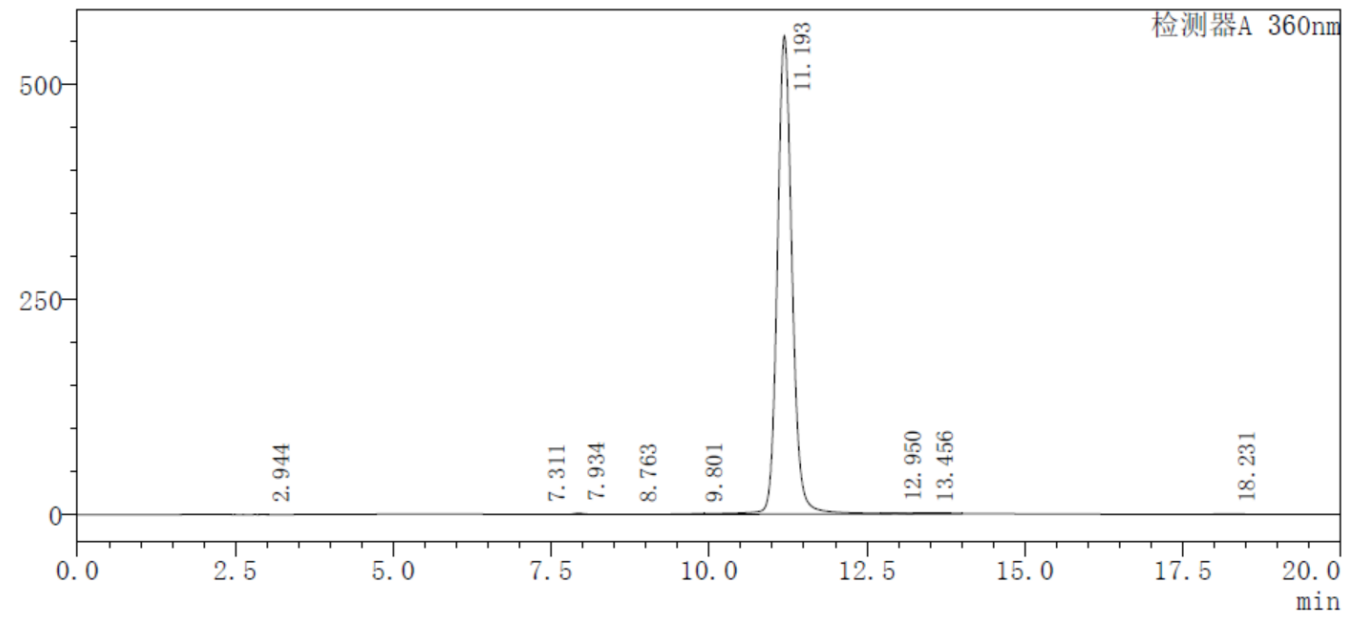

Figure S 17-4 HPLC Spectrum of $\mathbf{4 f}$

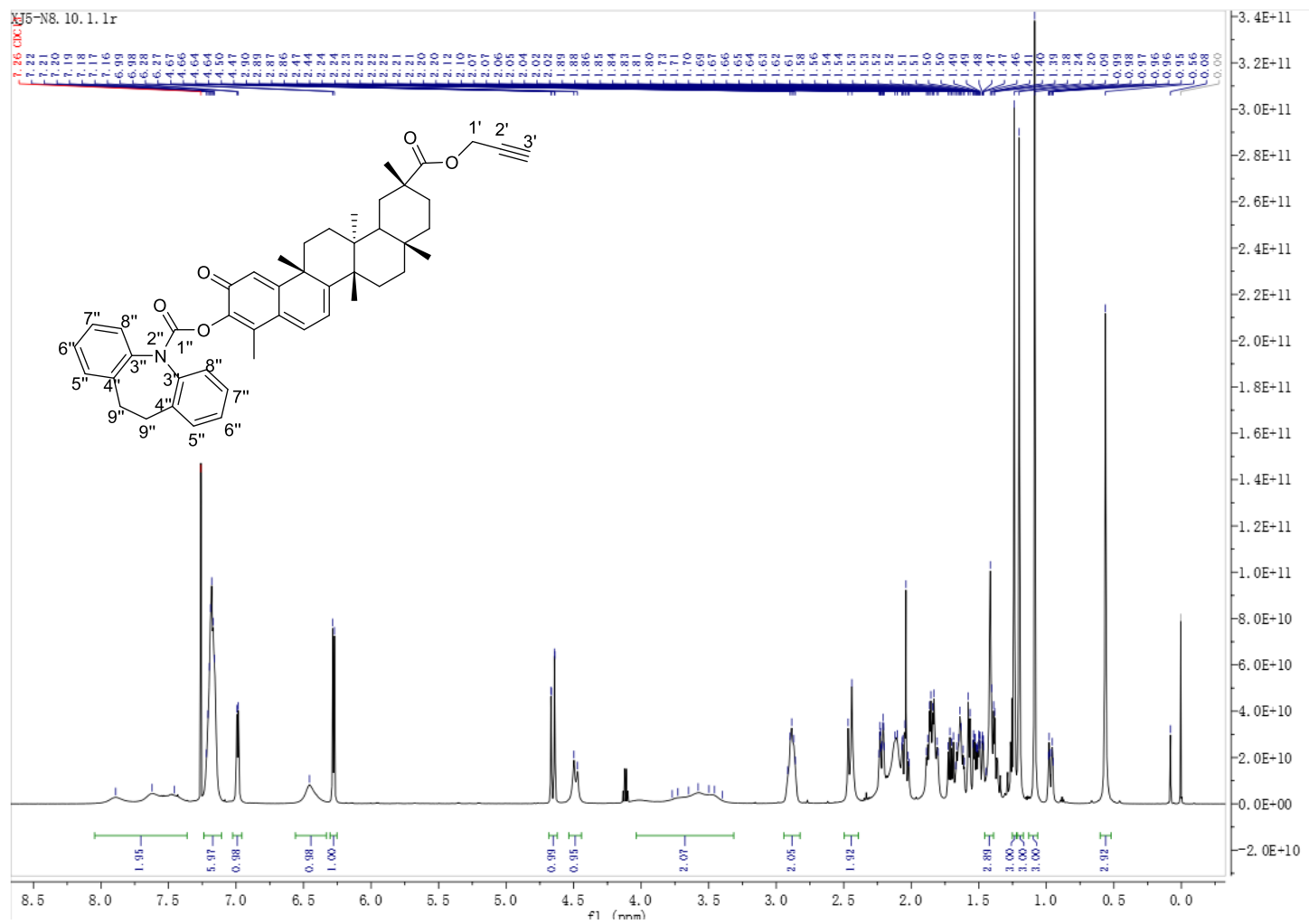

Figure $\mathbf{S}$ 18-1 ${ }^{1} \mathrm{H}$ NMR Spectrum $(600 \mathrm{MHz})$ of compound $\mathbf{4 g}$ in $\mathrm{CDCl}_{3}$ 


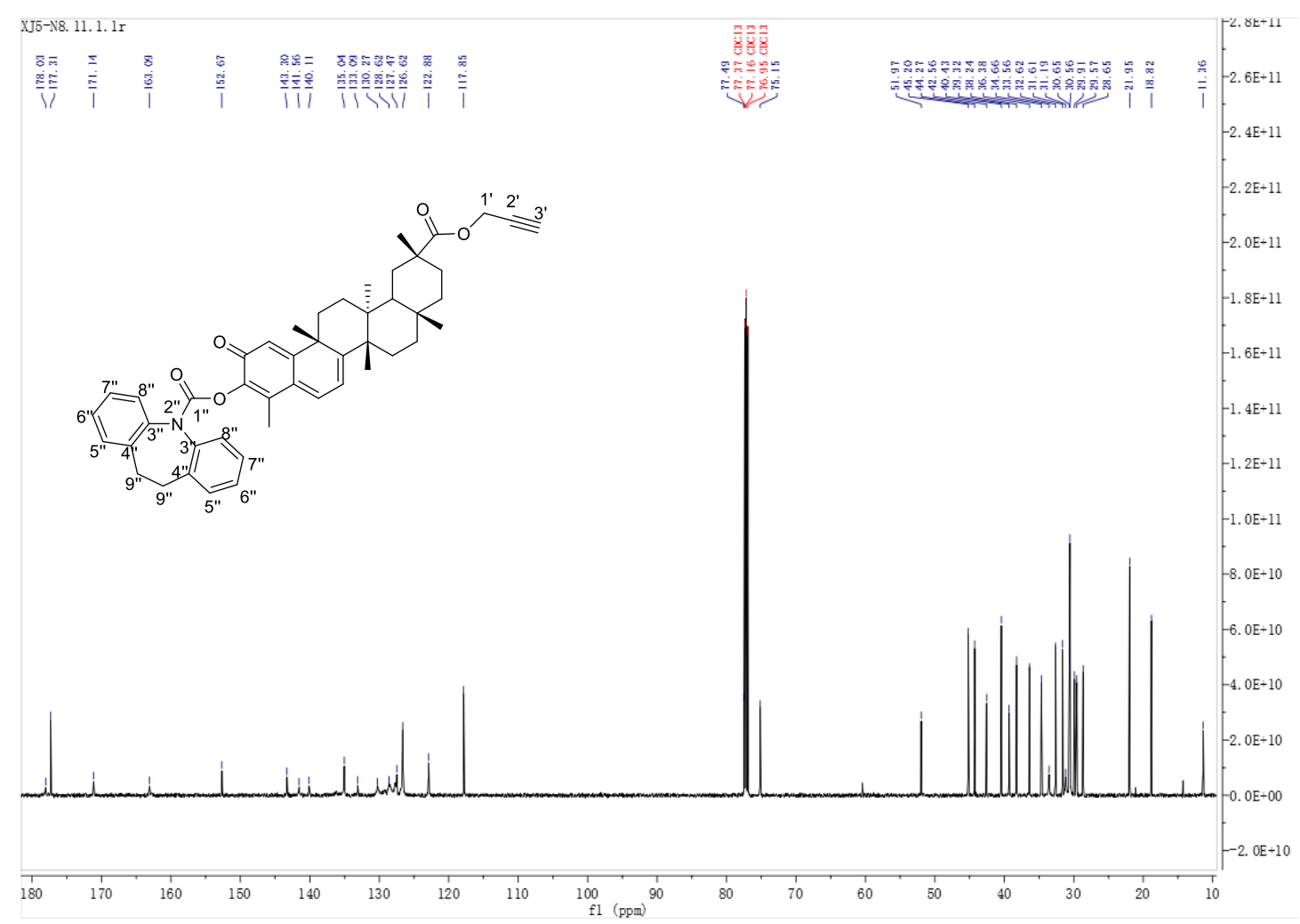

Figure S 18-2 ${ }^{13} \mathrm{C}$ NMR Spectrum $(150 \mathrm{MHz})$ of compound $\mathbf{4 g}$ in $\mathrm{CDCl}_{3}$

\section{Mass Spectrum List Report}

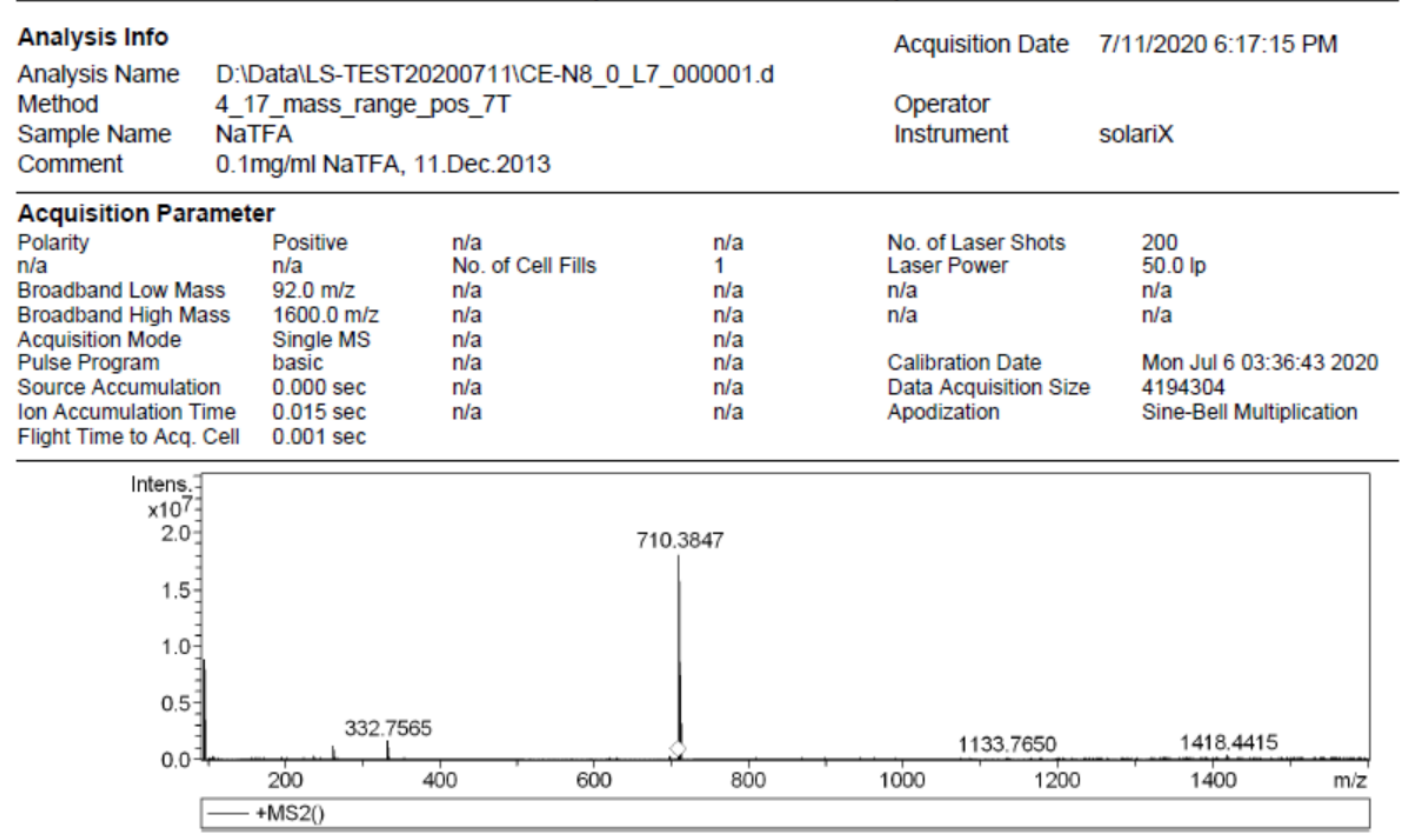

Figure S 18-3 HRESIMS Spectrum of $\mathbf{4 g}$ 
〈色谱图〉

$\mathrm{mV}$

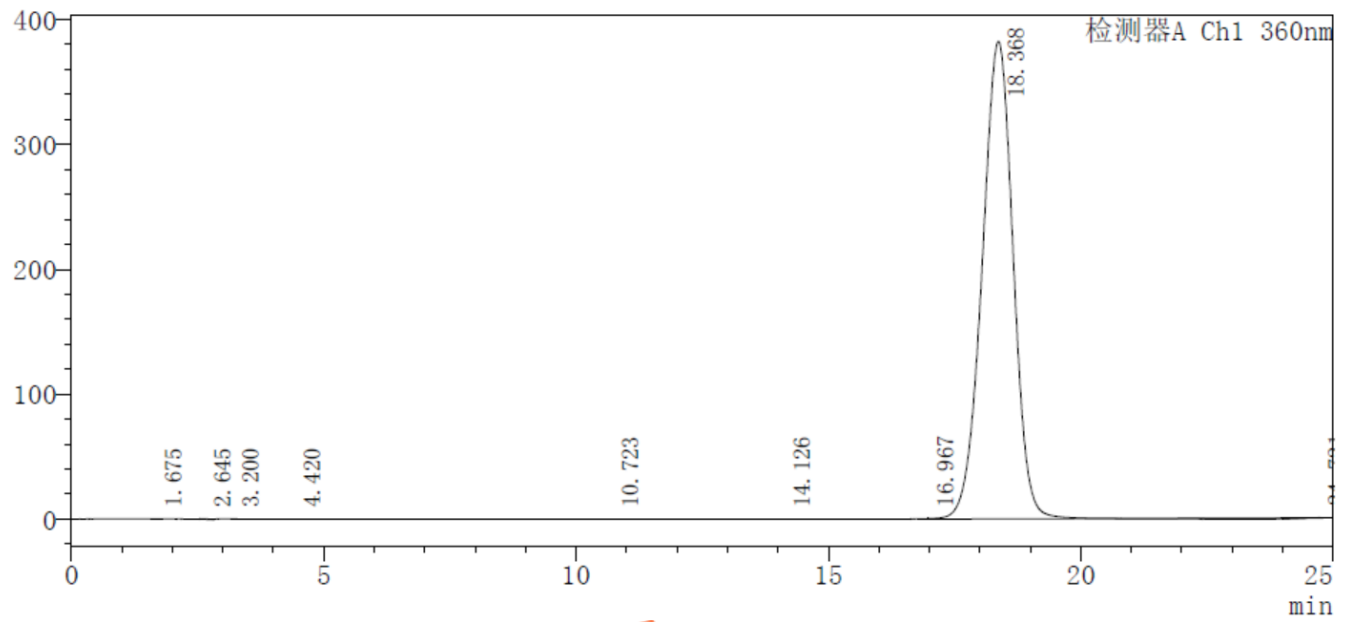

Figure S 18-4 HPLC Spectrum of $\mathbf{4 g}$

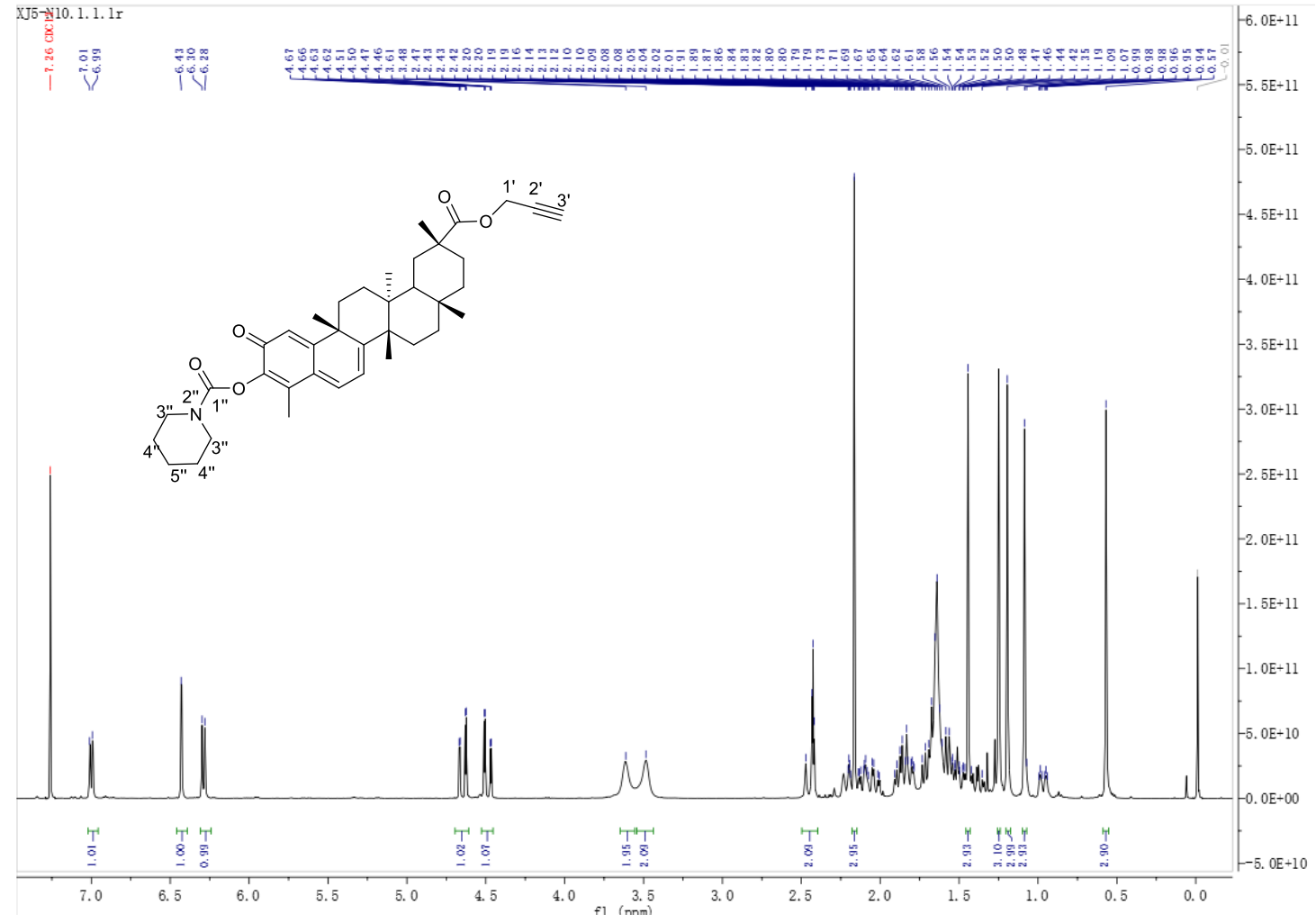

Figure S 19-1 ${ }^{1} \mathrm{H}$ NMR Spectrum $(600 \mathrm{MHz})$ of compound $4 \mathbf{h}$ in $\mathrm{CDCl}_{3}$ 


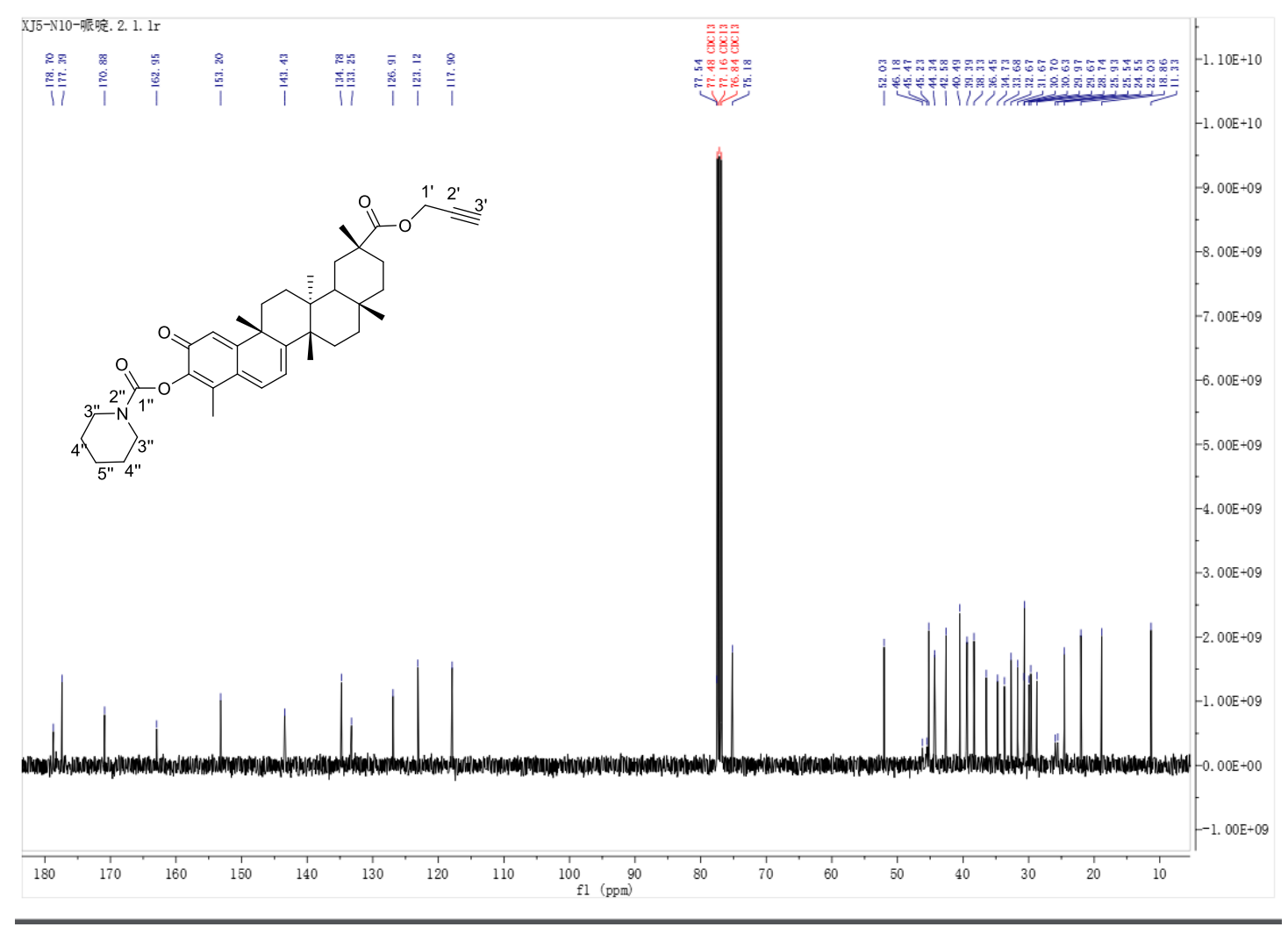

Figure S 19-2 ${ }^{13} \mathrm{C}$ NMR Spectrum $(150 \mathrm{MHz})$ of compound $\mathbf{4 h}$ in $\mathrm{CDCl}_{3}$ Mass Spectrum List Report

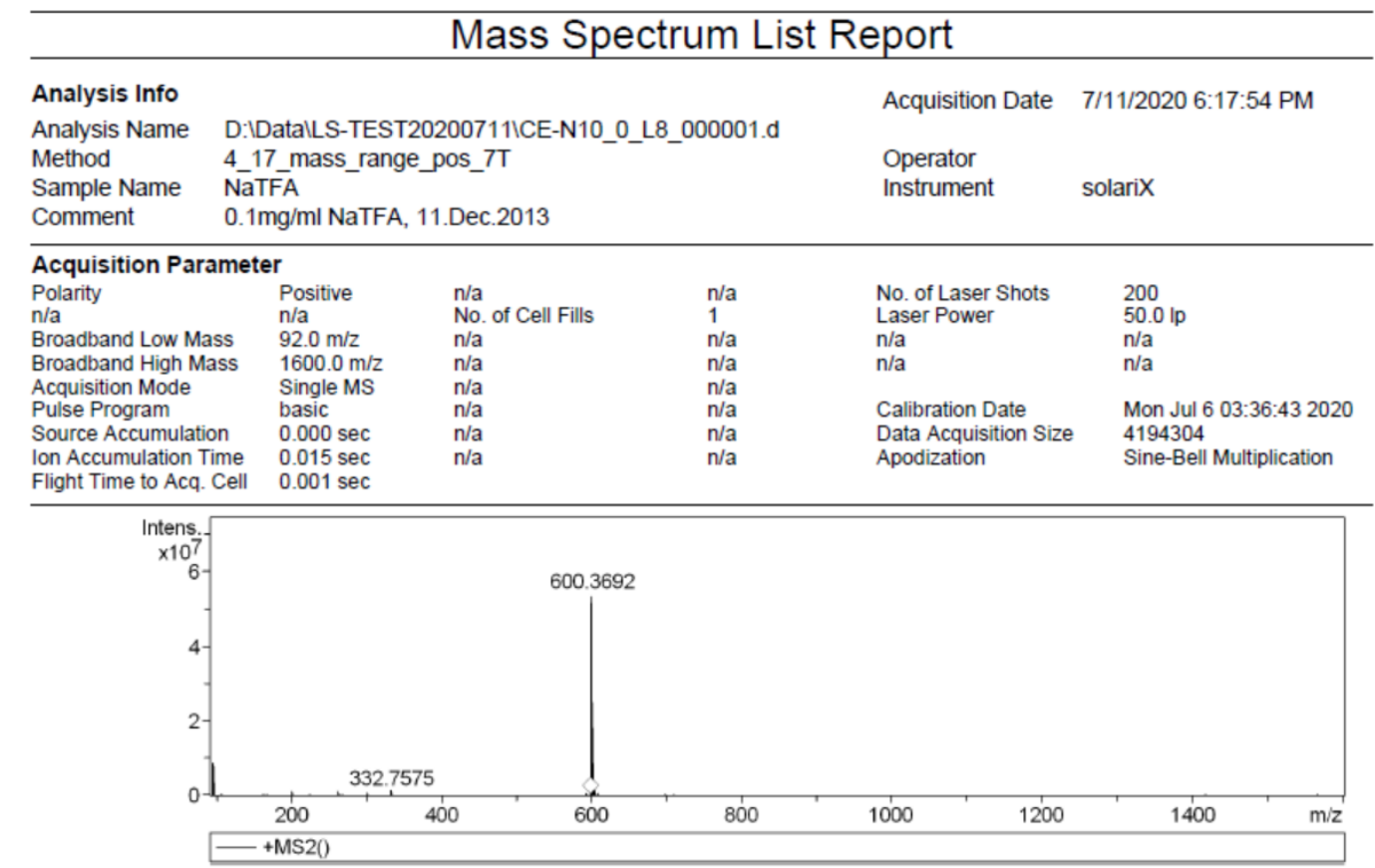

Figure S 19-3 HRESIMS Spectrum of $\mathbf{4 h}$ 


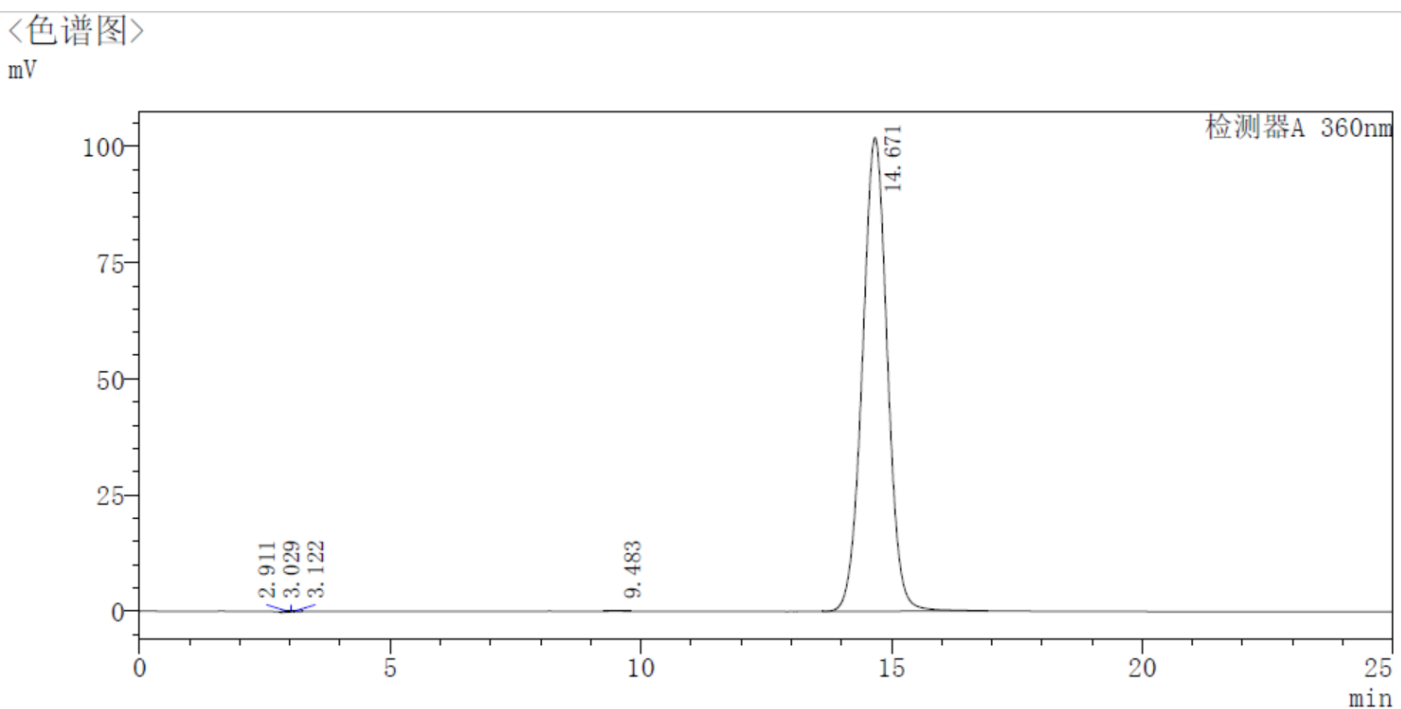

Figure S 19-4 HPLC Spectrum of $4 \mathrm{~h}$

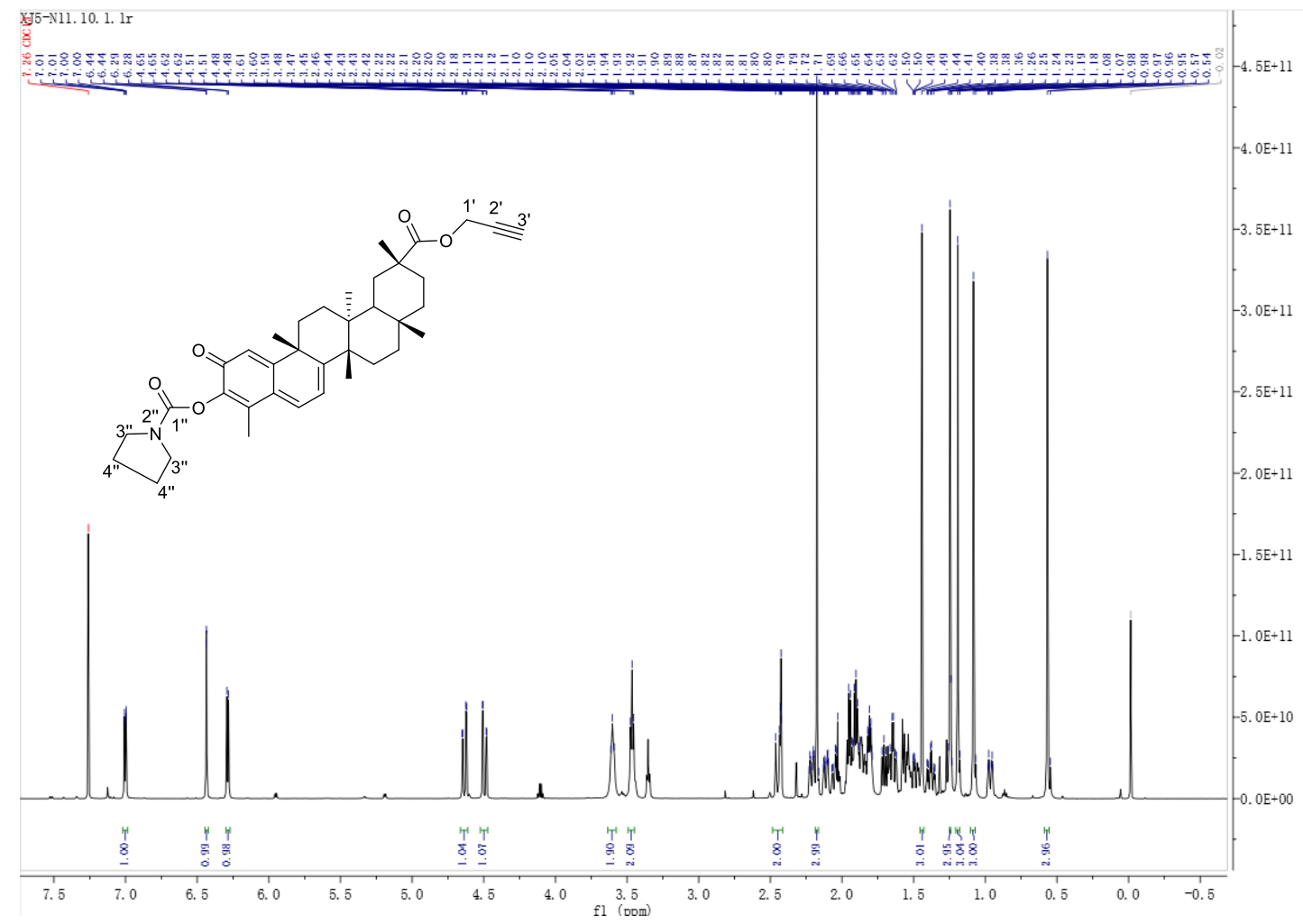

Figure S 20-1 ${ }^{1} \mathrm{H}$ NMR Spectrum $(600 \mathrm{MHz})$ of compound $4 \mathbf{i}$ in $\mathrm{CDCl}_{3}$ 


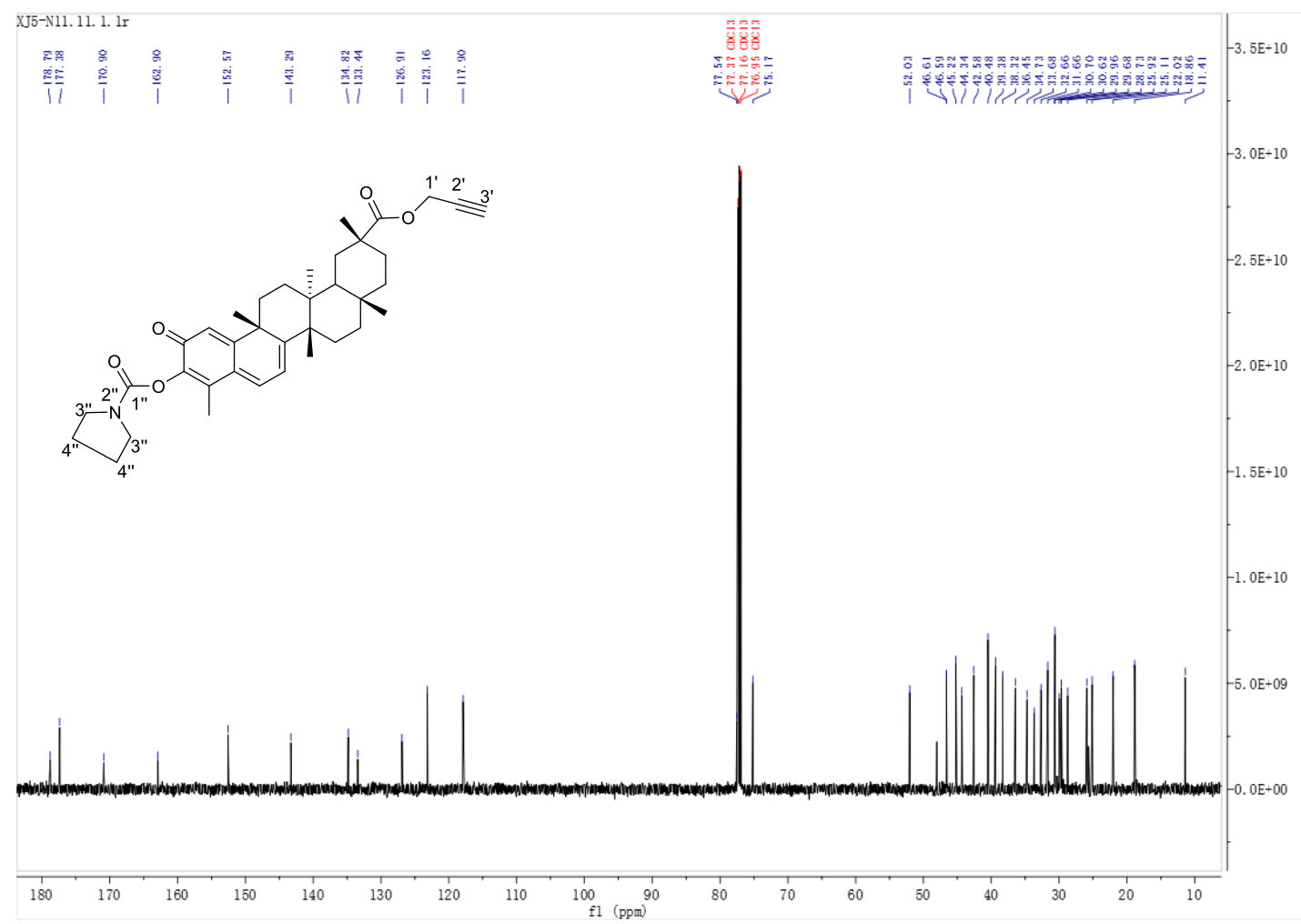

Figure S 20-2 ${ }^{13} \mathrm{C}$ NMR Spectrum $(150 \mathrm{MHz})$ of compound $4 \mathbf{i}$ in $\mathrm{CDCl}_{3}$

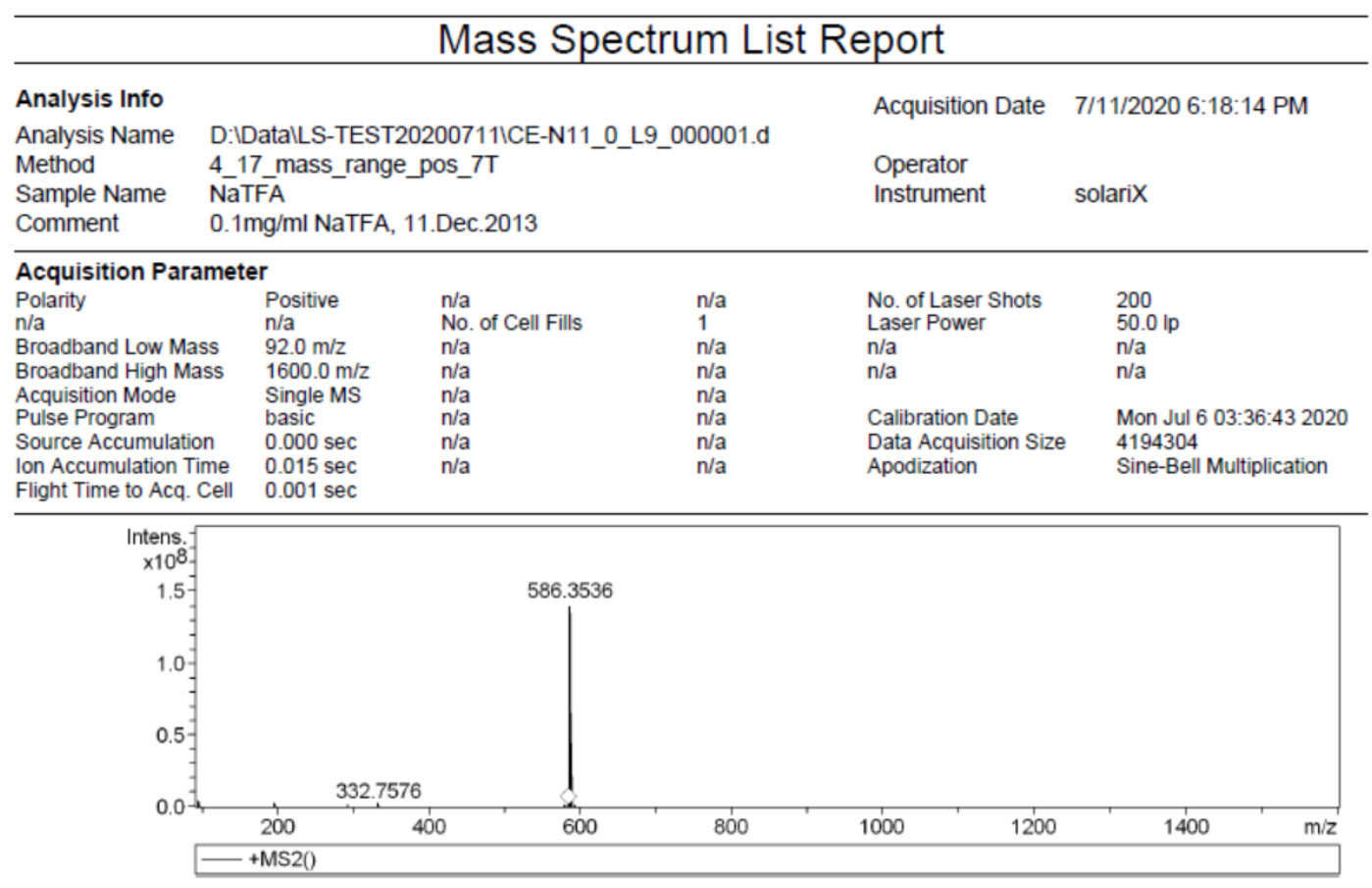

Figure S 20-3 HRESIMS Spectrum of $\mathbf{4 i}$ 
〈色谱图〉

$\mathrm{mV}$

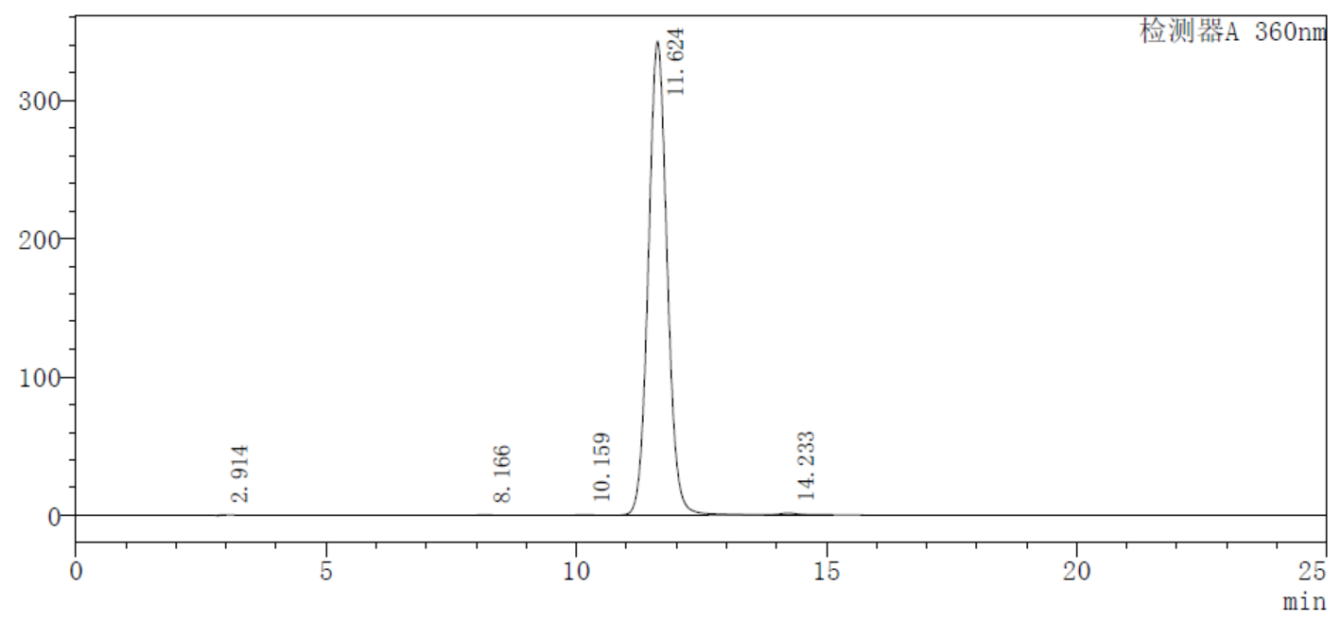

Figure S 20-4 HPLC Spectrum of $\mathbf{4 i}$

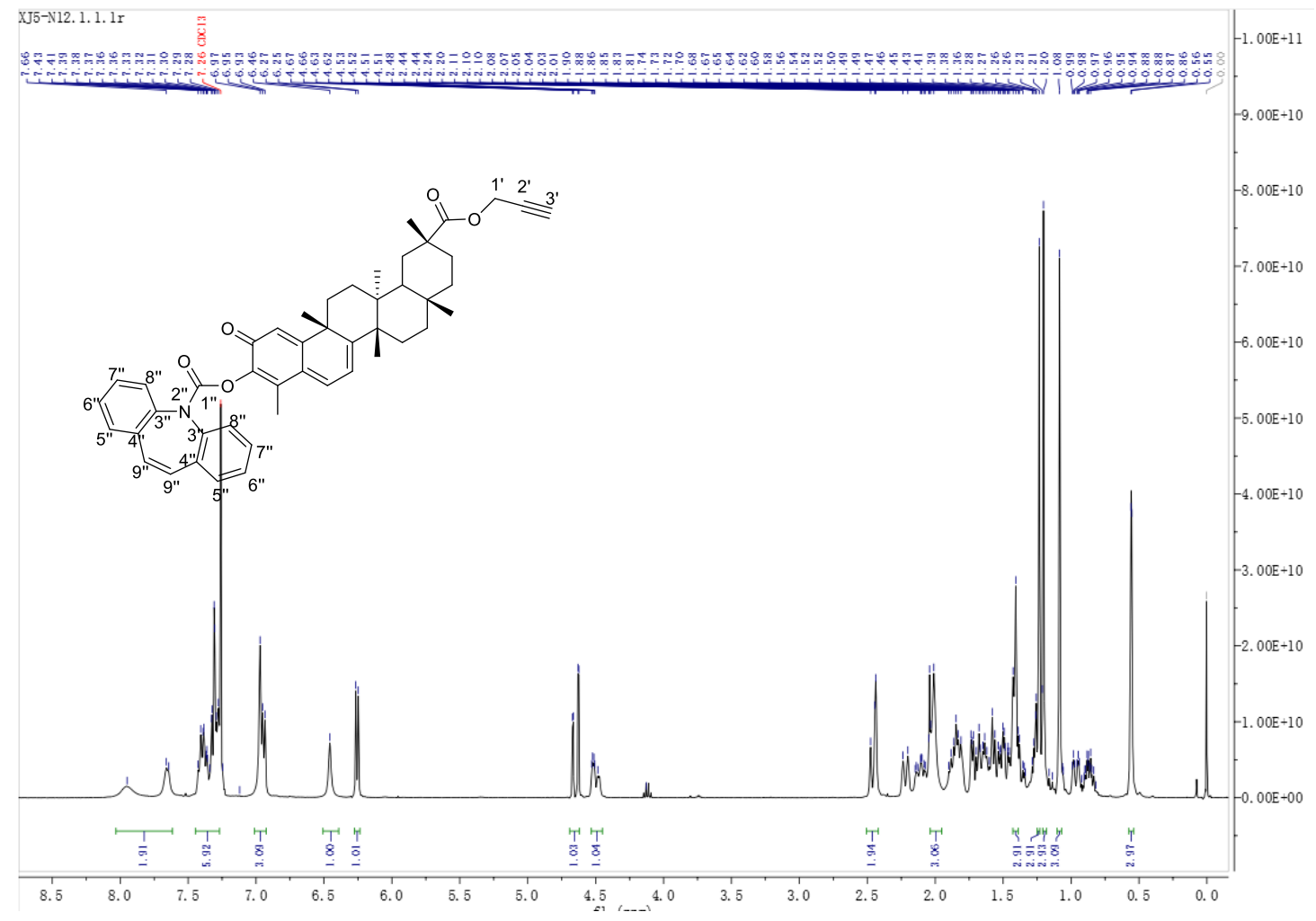

Figure S 21-1 ${ }^{1} \mathrm{H}$ NMR Spectrum $(600 \mathrm{MHz})$ of compound $\mathbf{4 j}$ in $\mathrm{CDCl}_{3}$ 


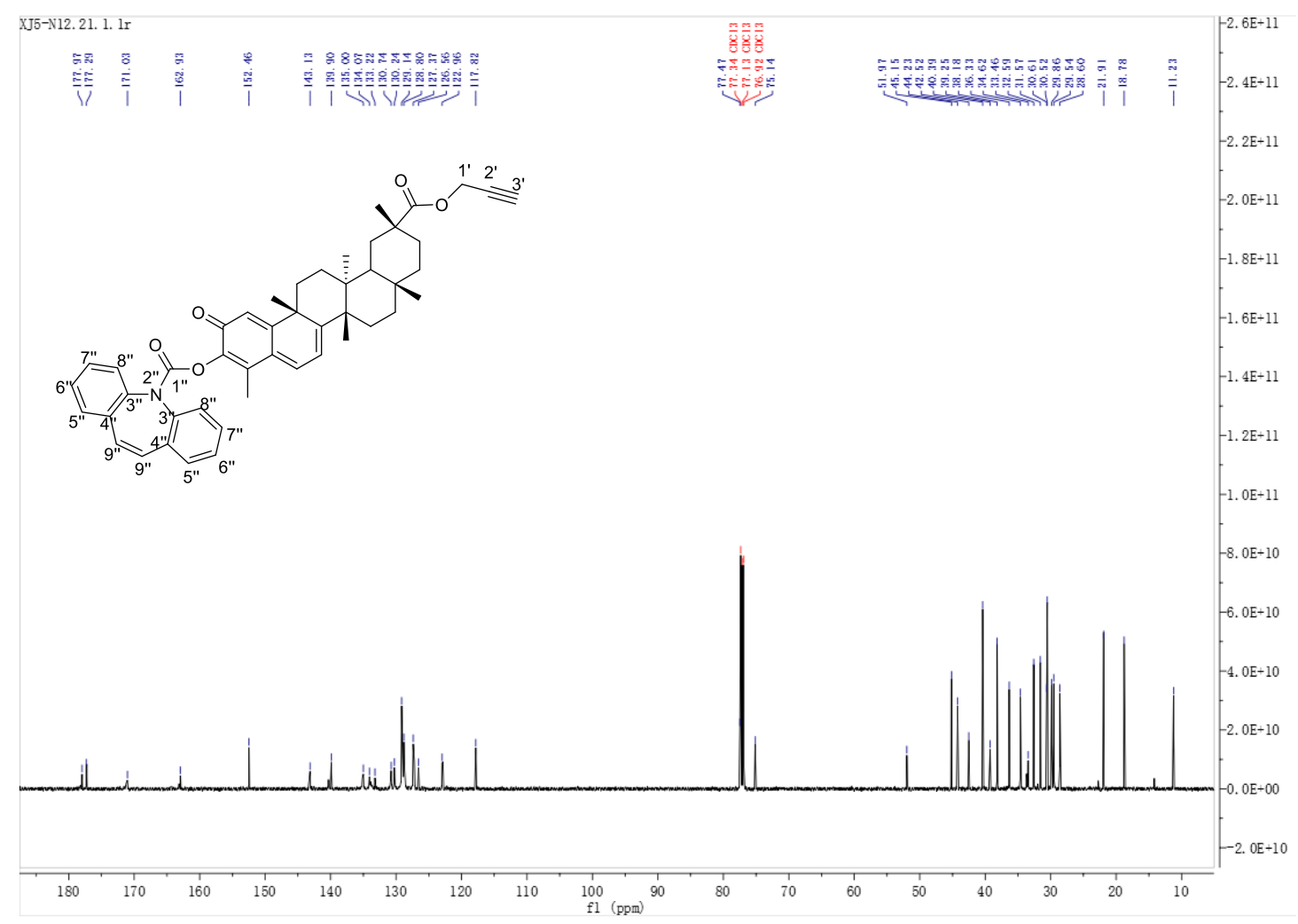

Figure $\mathbf{S}$ 21-2 ${ }^{13} \mathrm{C}$ NMR Spectrum $(150 \mathrm{MHz})$ of compound $\mathbf{4 j}$ in $\mathrm{CDCl}_{3}$

\section{Mass Spectrum List Report}

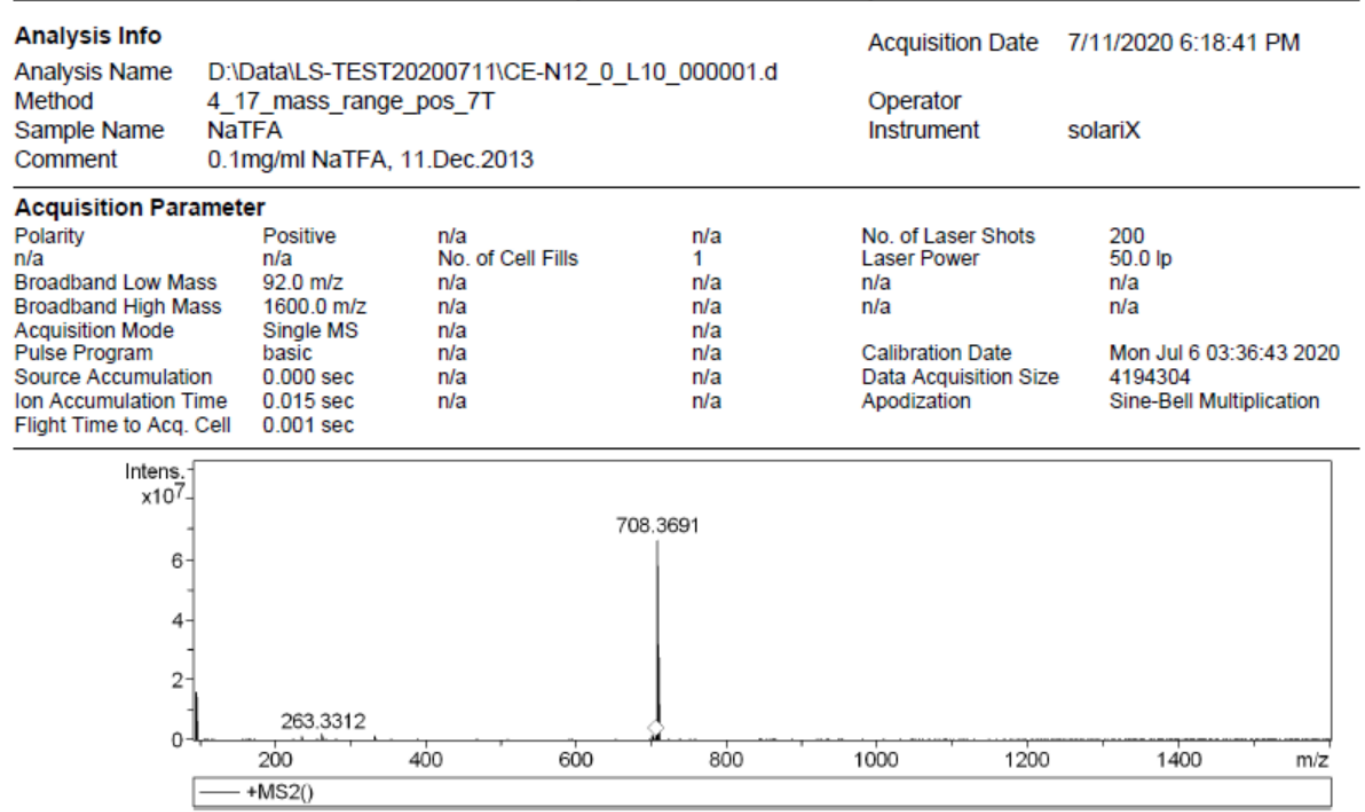

Figure S 21-3 HRESIMS Spectrum of $\mathbf{4 j}$ 
〈色谱图〉

$\mathrm{mV}$

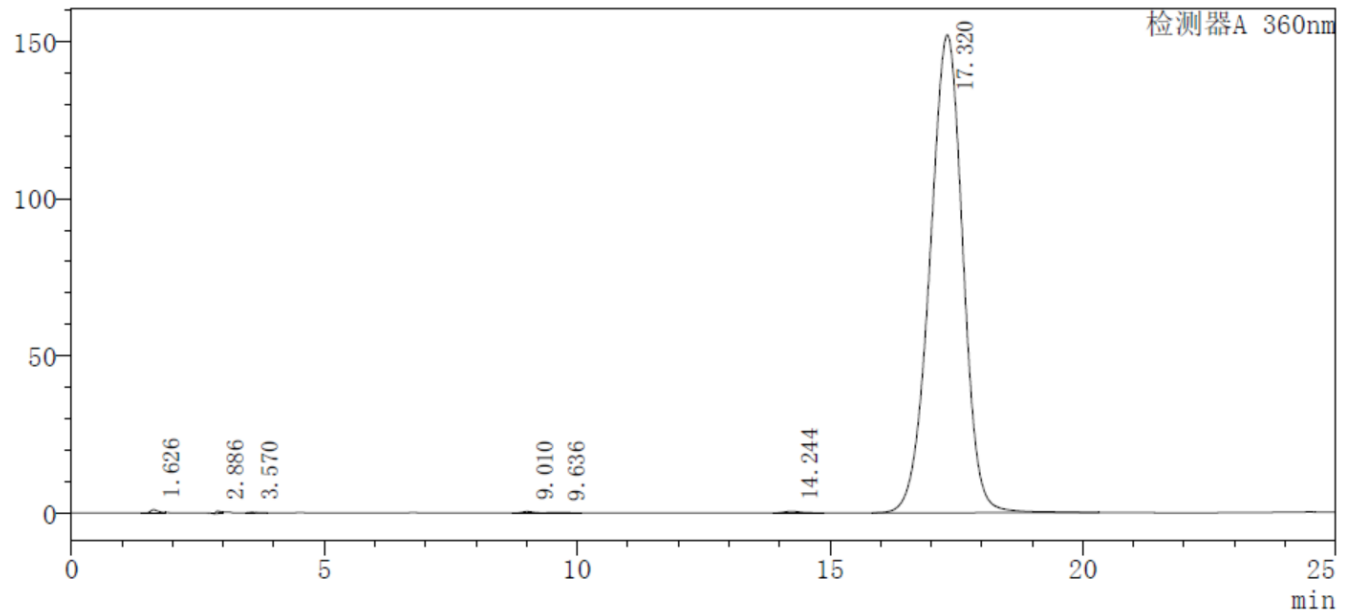

Figure S 21-4 HPLC Spectrum of $\mathbf{4 j}$

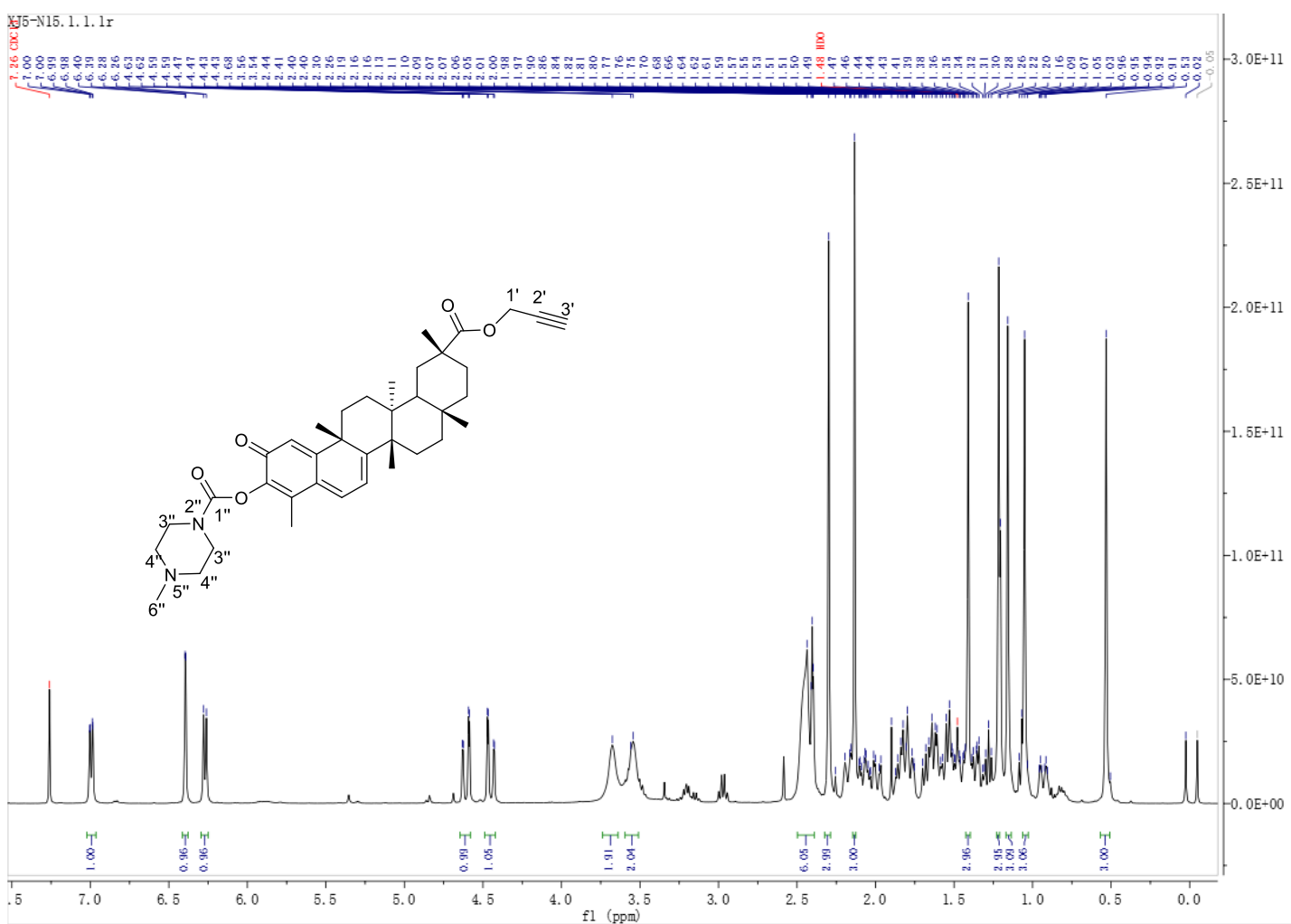

Figure S 22-1 ${ }^{1} \mathrm{H}$ NMR Spectrum $(600 \mathrm{MHz})$ of compound $\mathbf{4 k}$ in $\mathrm{CDCl}_{3}$ 


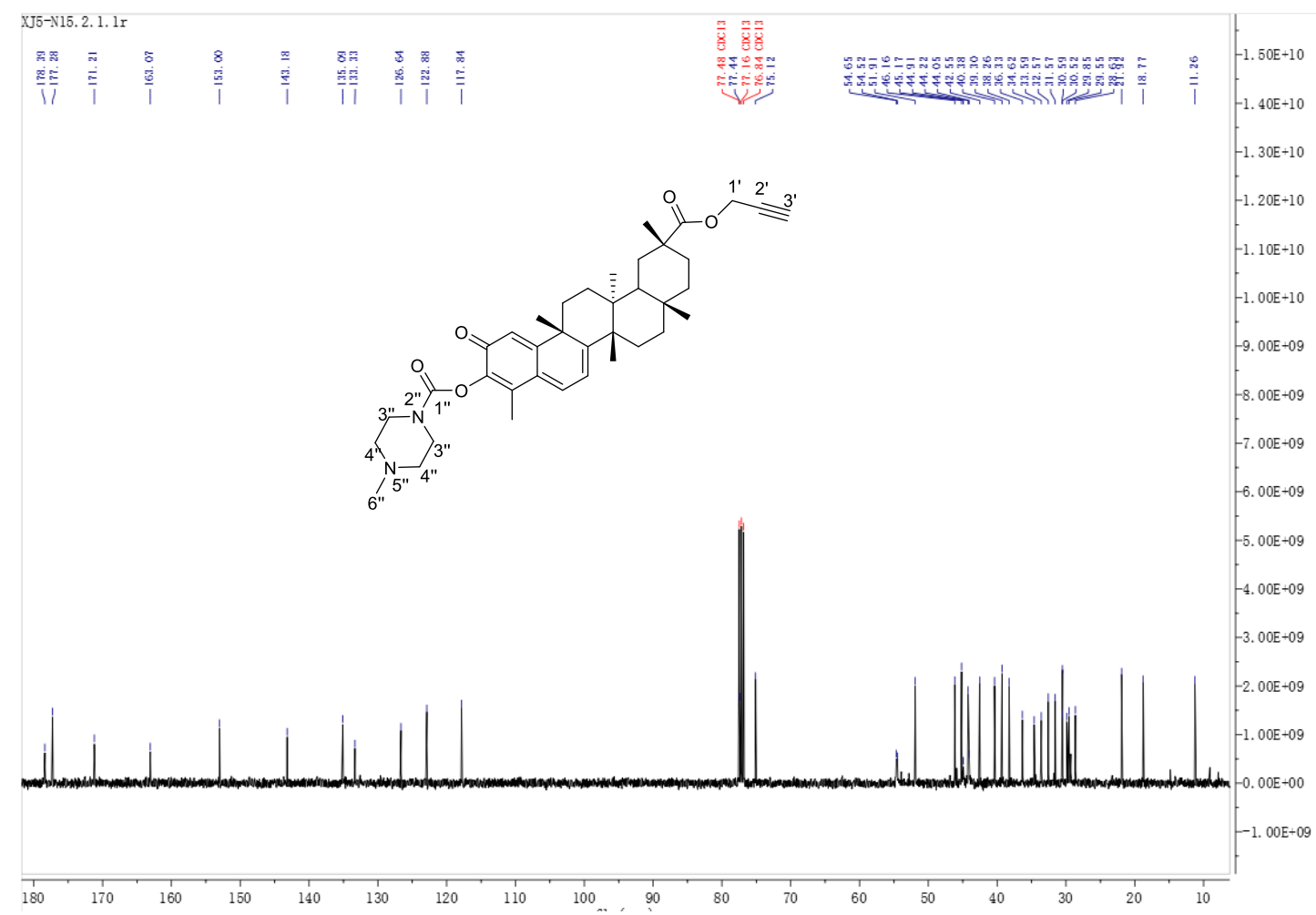

Figure S 22-2 ${ }^{13} \mathrm{C}$ NMR Spectrum $(150 \mathrm{MHz})$ of compound $4 \mathbf{k}$ in $\mathrm{CDCl}_{3}$

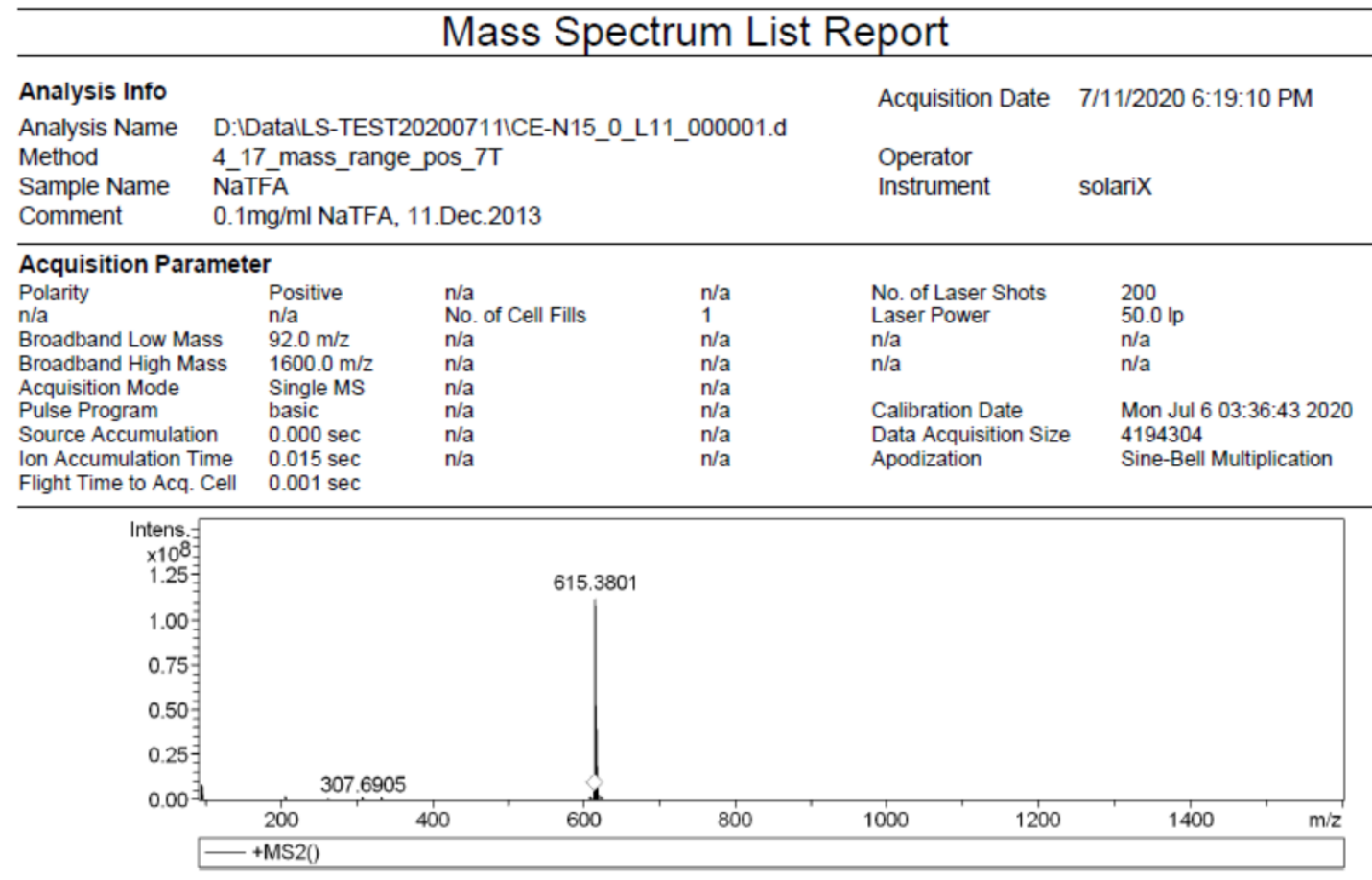

Figure S 22-3 HRESIMS Spectrum of $\mathbf{4 k}$ 


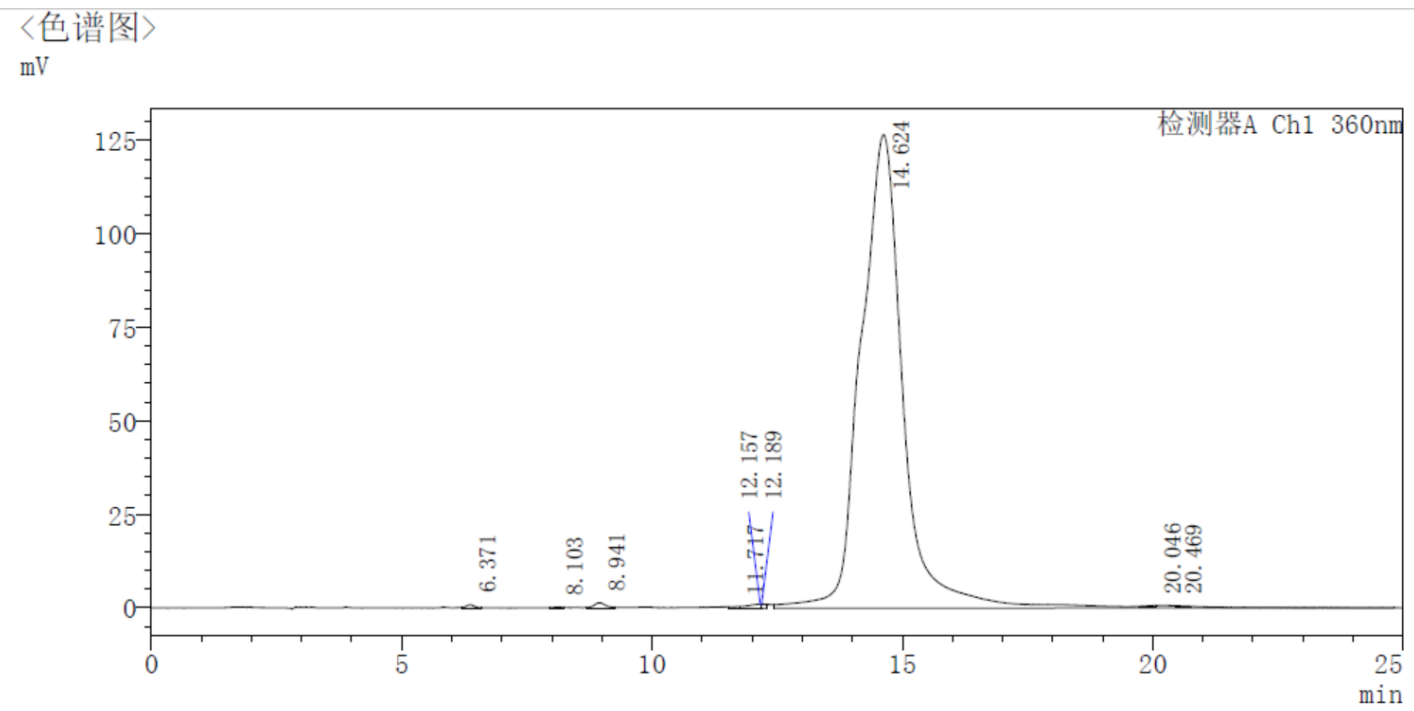

Figure S 22-4 HPLC Spectrum of $4 \mathbf{k}$

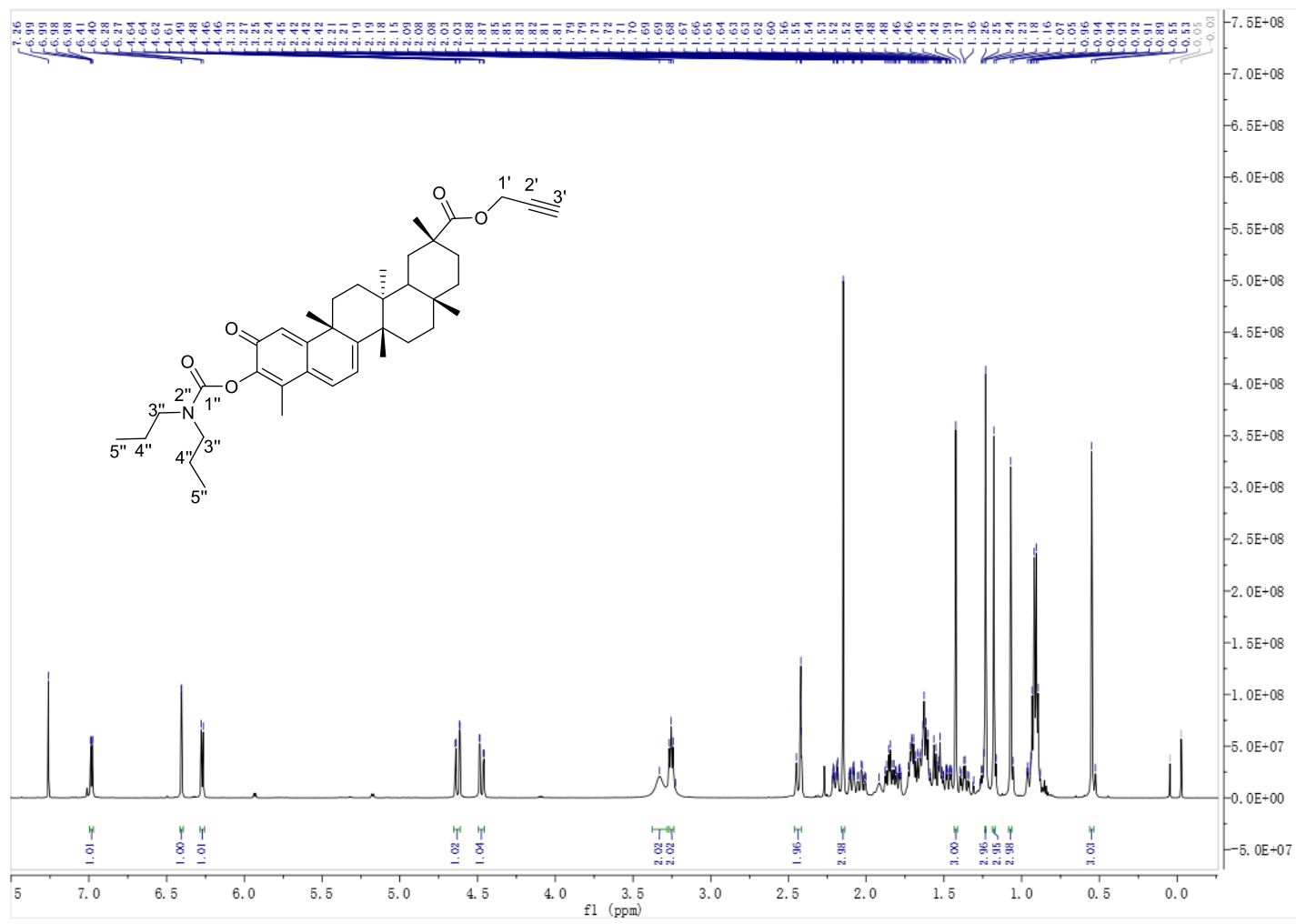

Figure S 23-1 ${ }^{1} \mathrm{H}$ NMR Spectrum $(600 \mathrm{MHz})$ of compound $4 \mathbf{l}$ in $\mathrm{CDCl}_{3}$ 


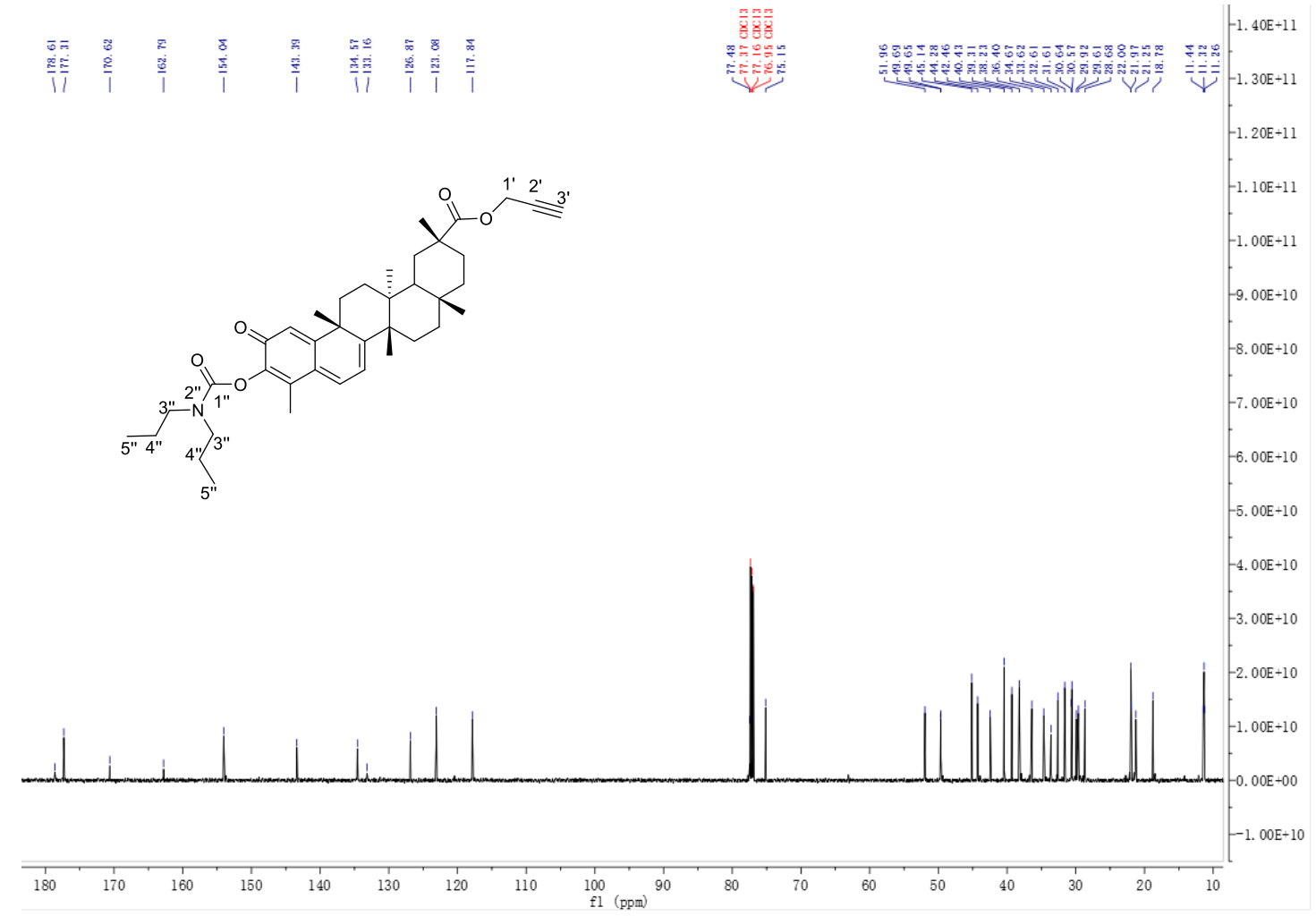

Figure S 23-2 ${ }^{13} \mathrm{C}$ NMR Spectrum $(150 \mathrm{MHz})$ of compound $4 \mathrm{l}$ in $\mathrm{CDCl}_{3}$

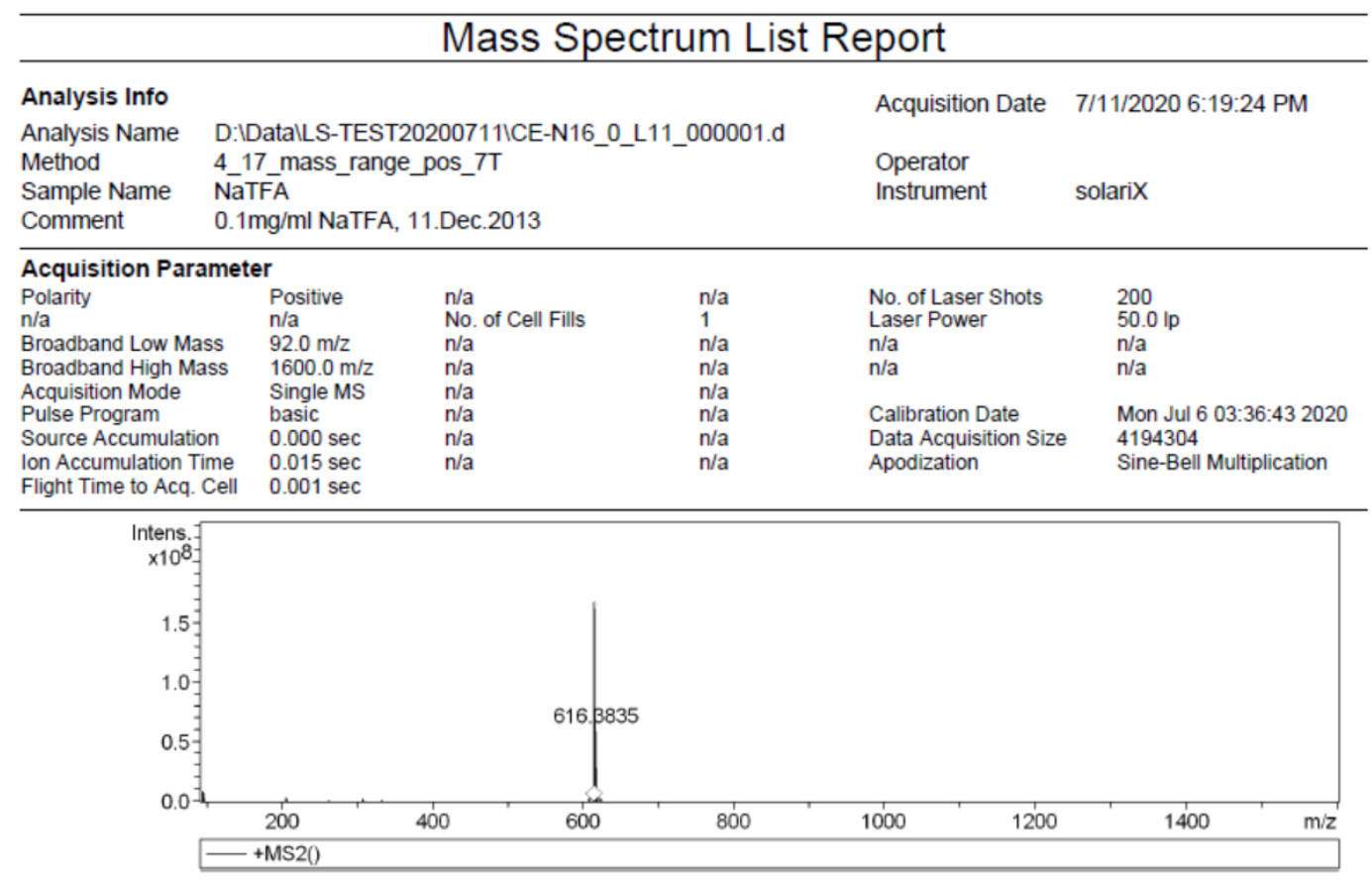

Figure S 23-3 HRESIMS Spectrum of 4l 
〈色谱图〉

$\mathrm{mV}$

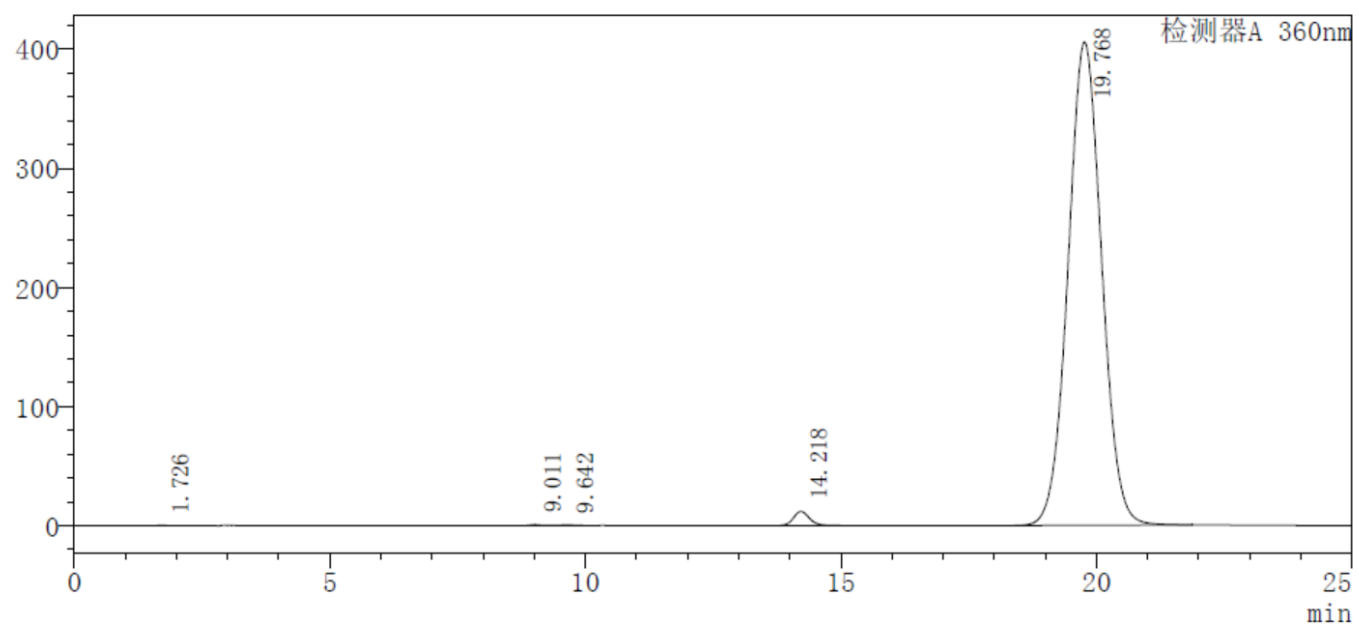

Figure S 23-4 HPLC Spectrum of 41

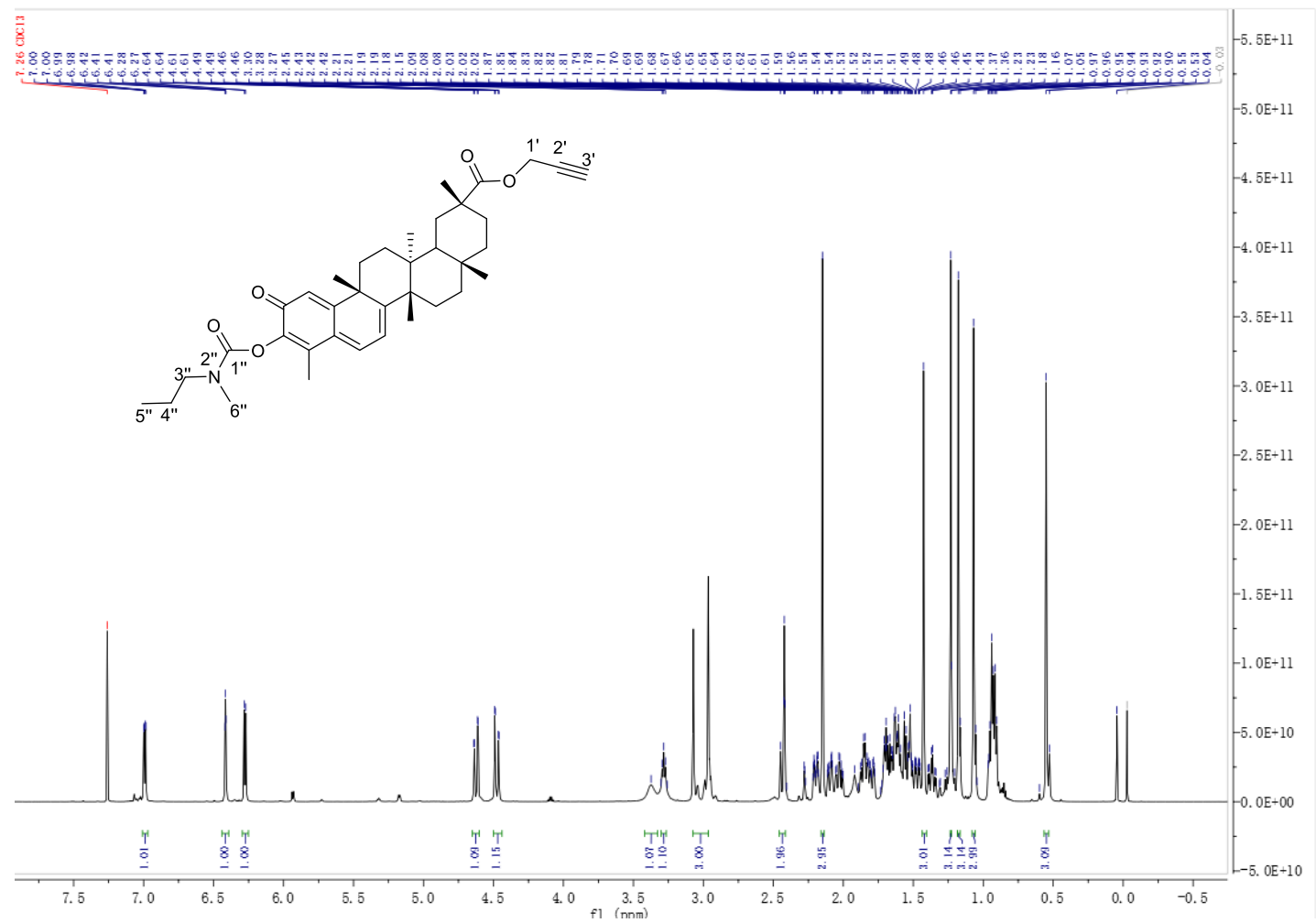

Figure S 24-1 ${ }^{1} \mathrm{H}$ NMR Spectrum $(600 \mathrm{MHz})$ of compound $\mathbf{4 m}$ in $\mathrm{CDCl}_{3}$ 


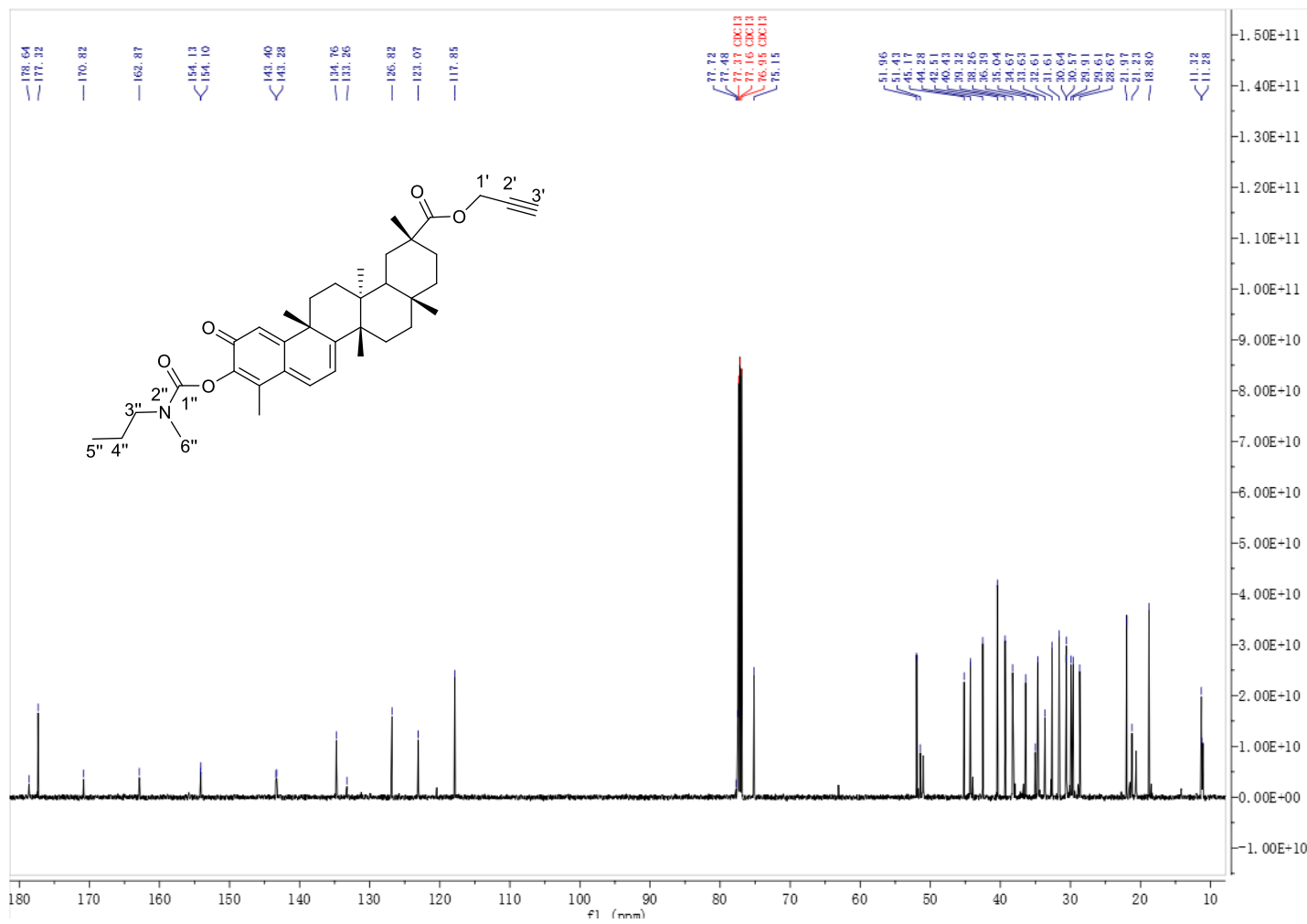

Figure S 24-2 ${ }^{13} \mathrm{C}$ NMR Spectrum $(150 \mathrm{MHz})$ of compound $\mathbf{4 m}$ in $\mathrm{CDCl}_{3}$

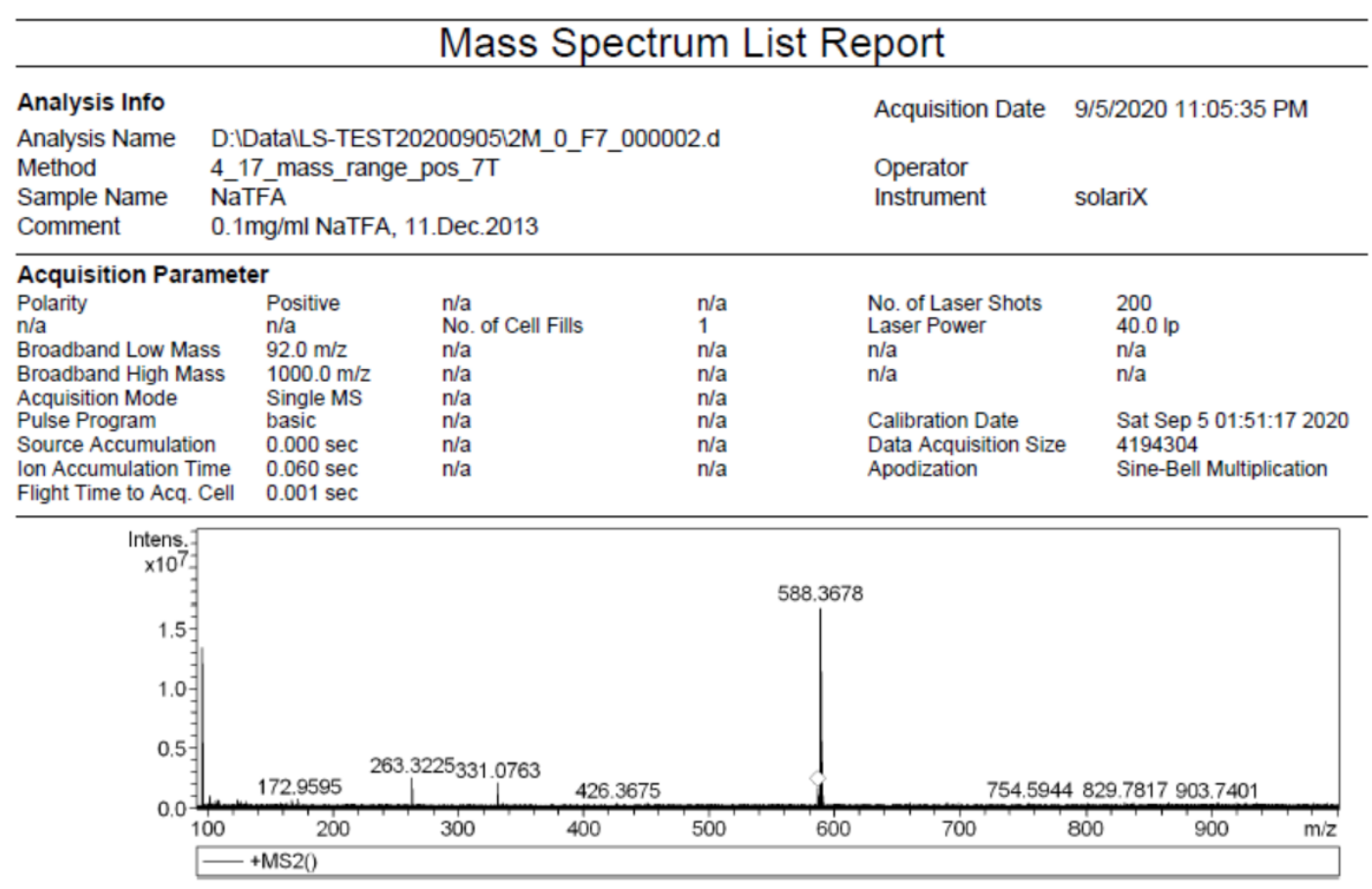

Figure S 24-3 HRESIMS Spectrum of $\mathbf{4 m}$ 


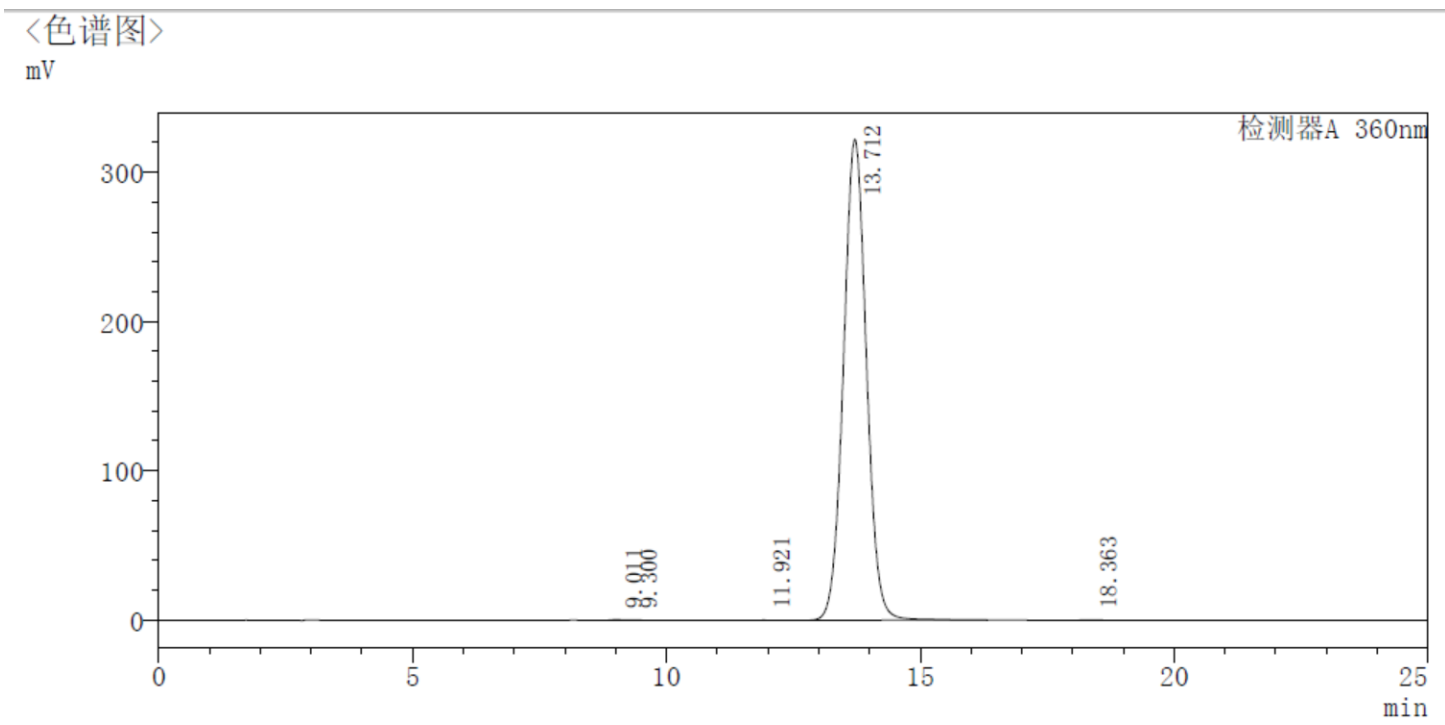

Figure S 24-4 HPLC Spectrum of 4m

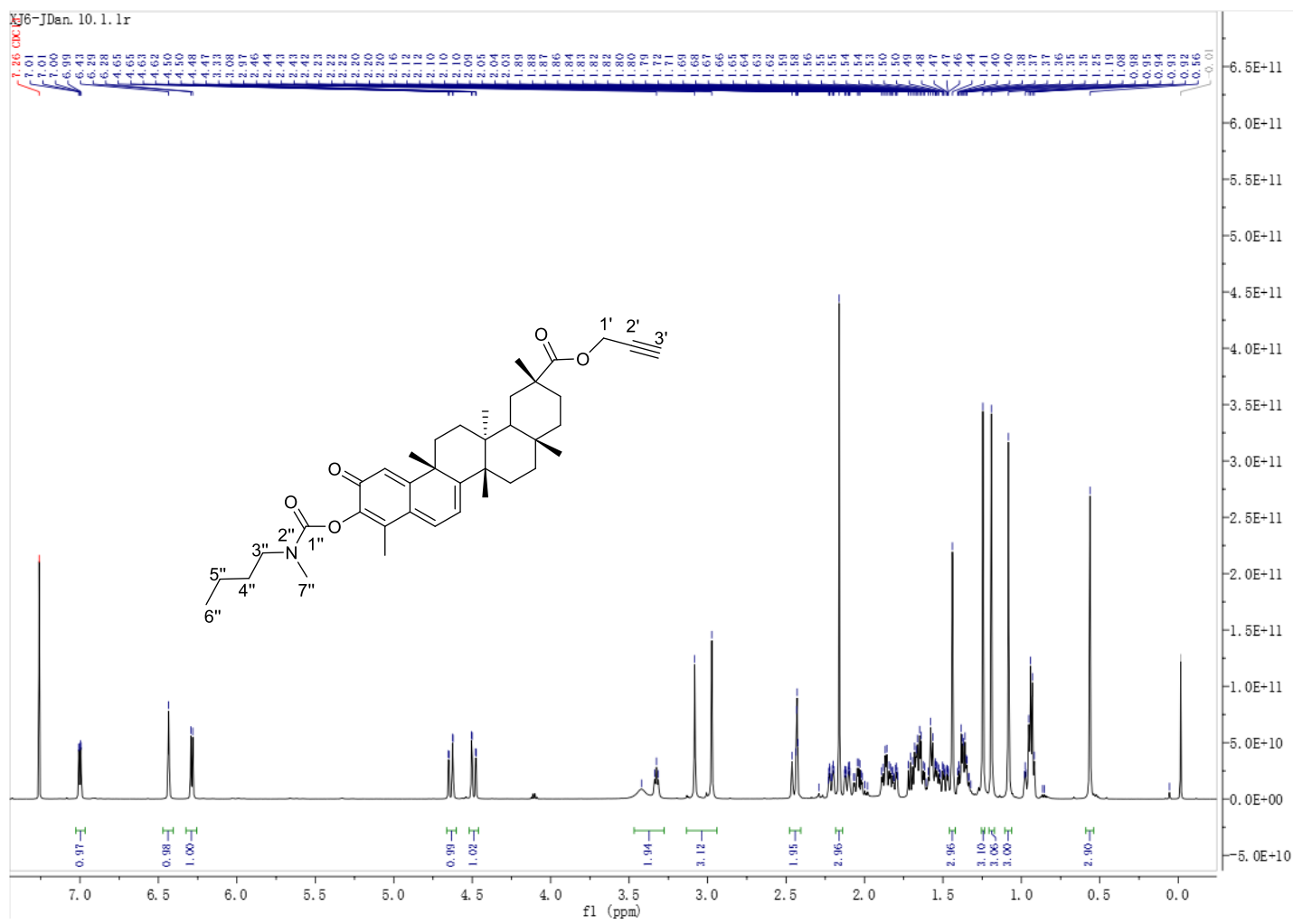

Figure S 25-1 ${ }^{1} \mathrm{H}$ NMR Spectrum $(600 \mathrm{MHz})$ of compound $4 \mathbf{n}$ in $\mathrm{CDCl}_{3}$ 


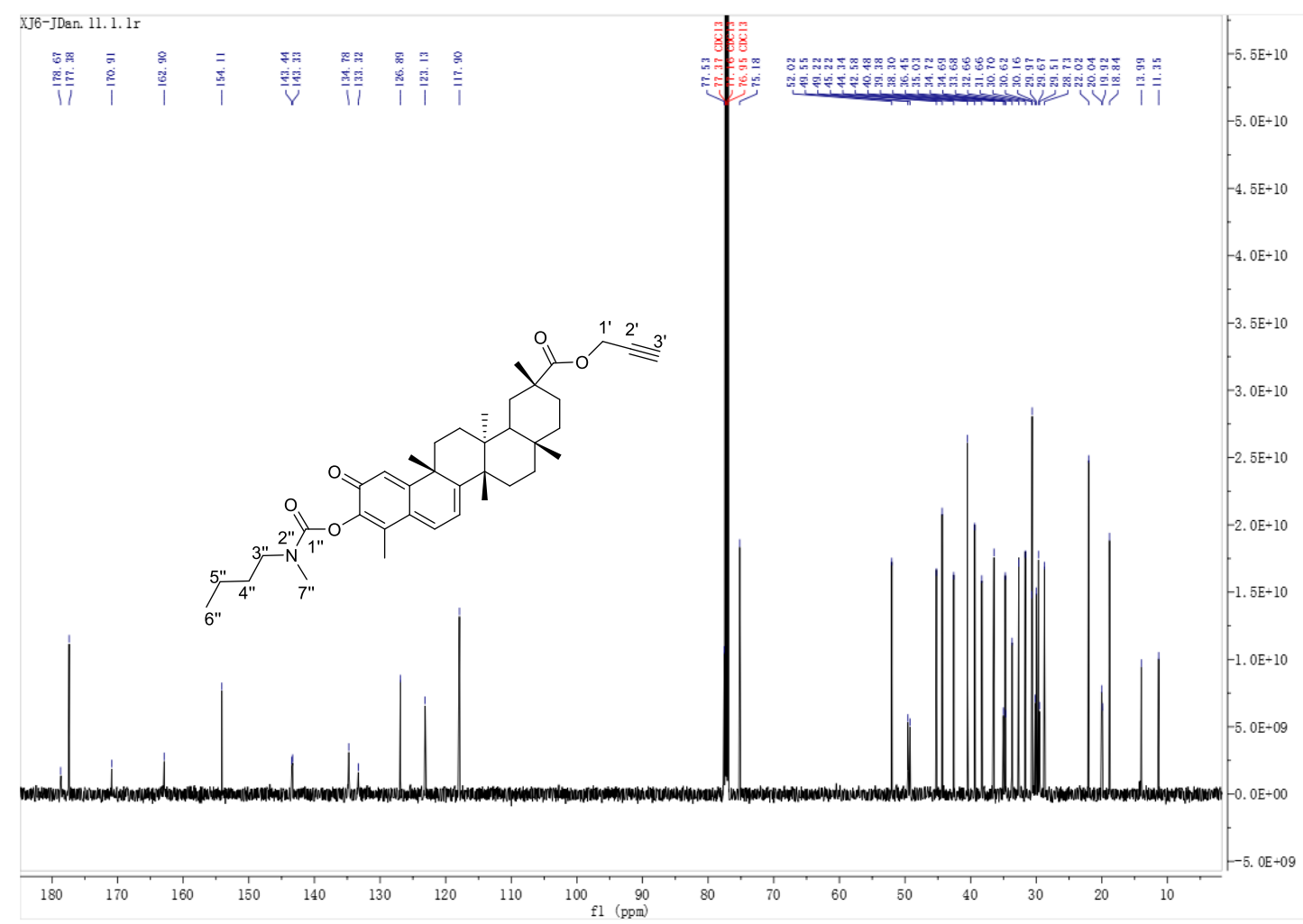

Figure S 25-2 ${ }^{13} \mathrm{C}$ NMR Spectrum $(150 \mathrm{MHz})$ of compound $4 \mathbf{n}$ in $\mathrm{CDCl}_{3}$

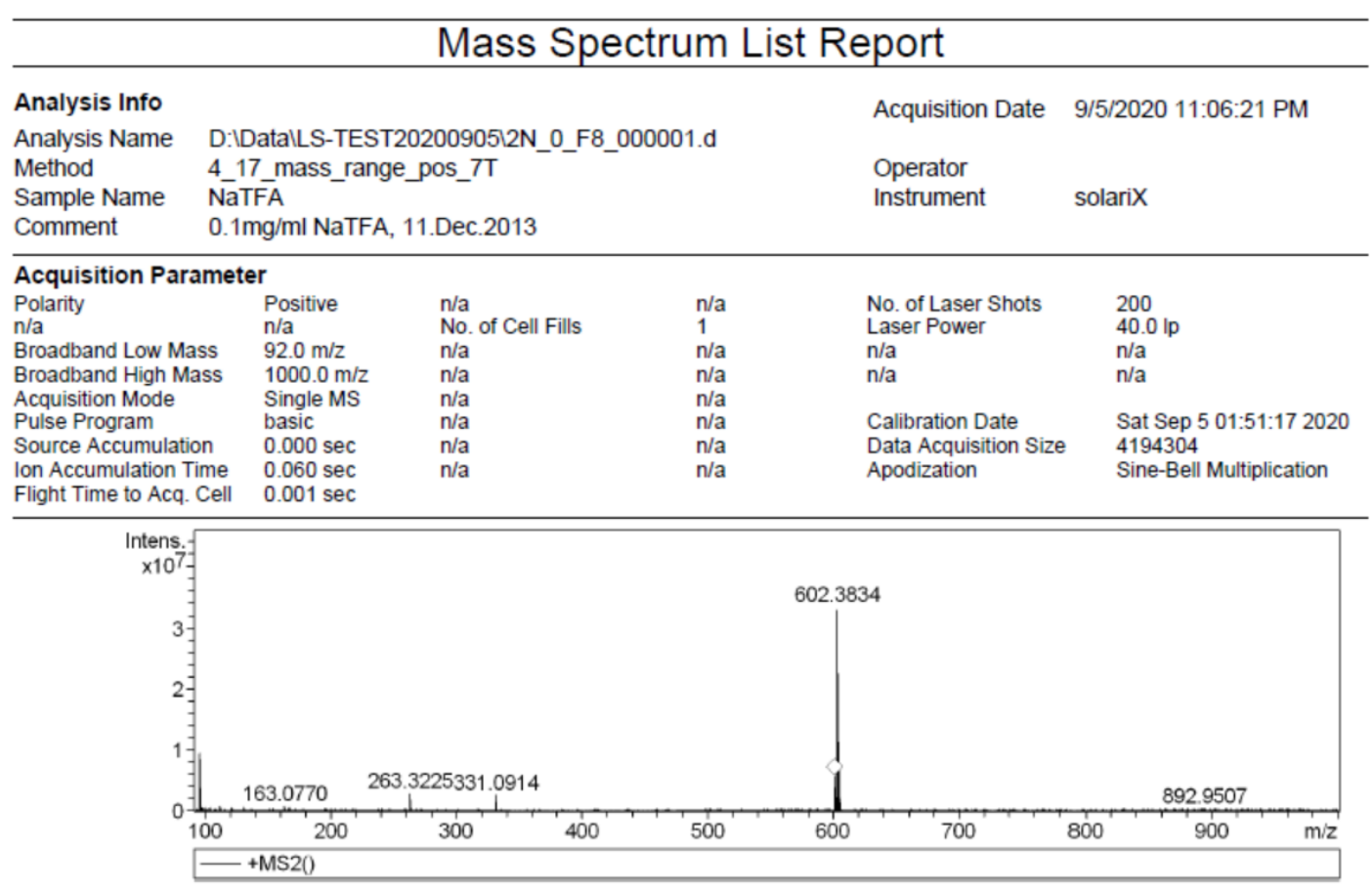

Figure S 25-3 HRESIMS Spectrum of 4n 


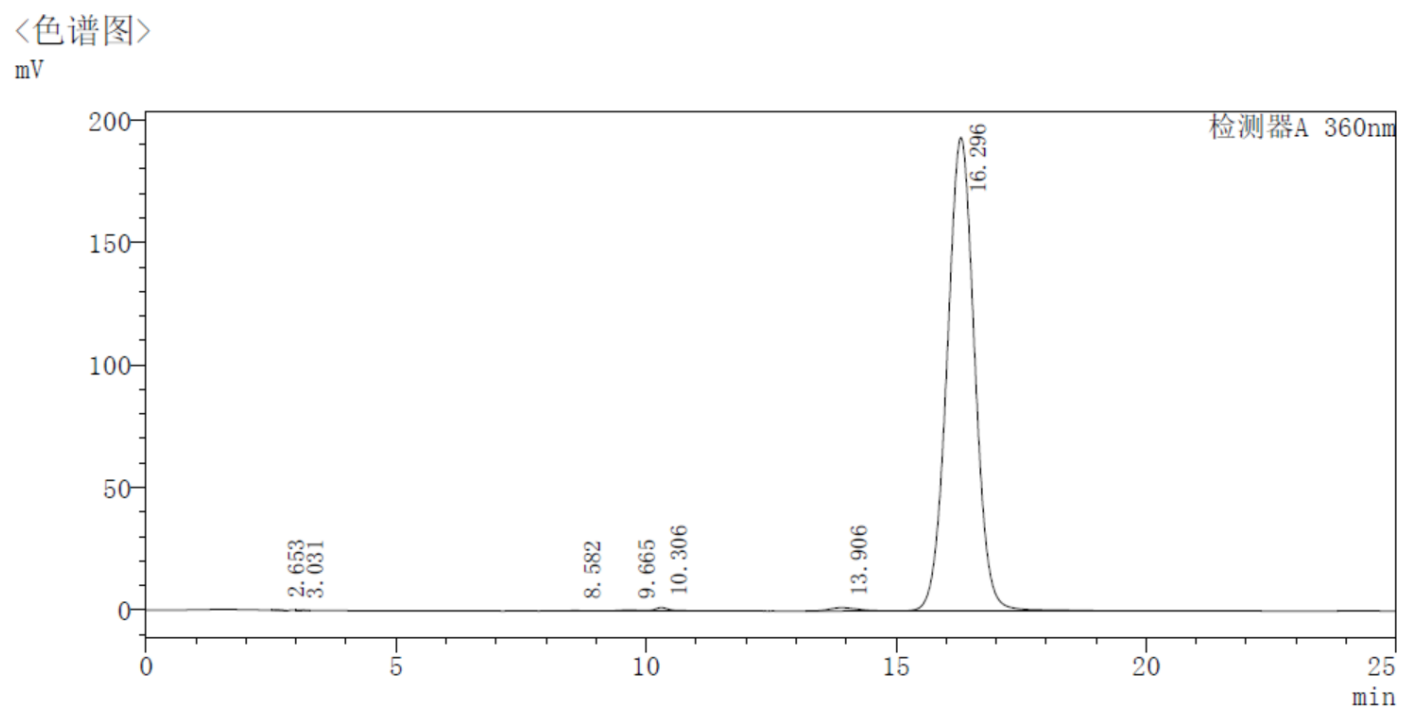

Figure S 25-4 HPLC Spectrum of 4n

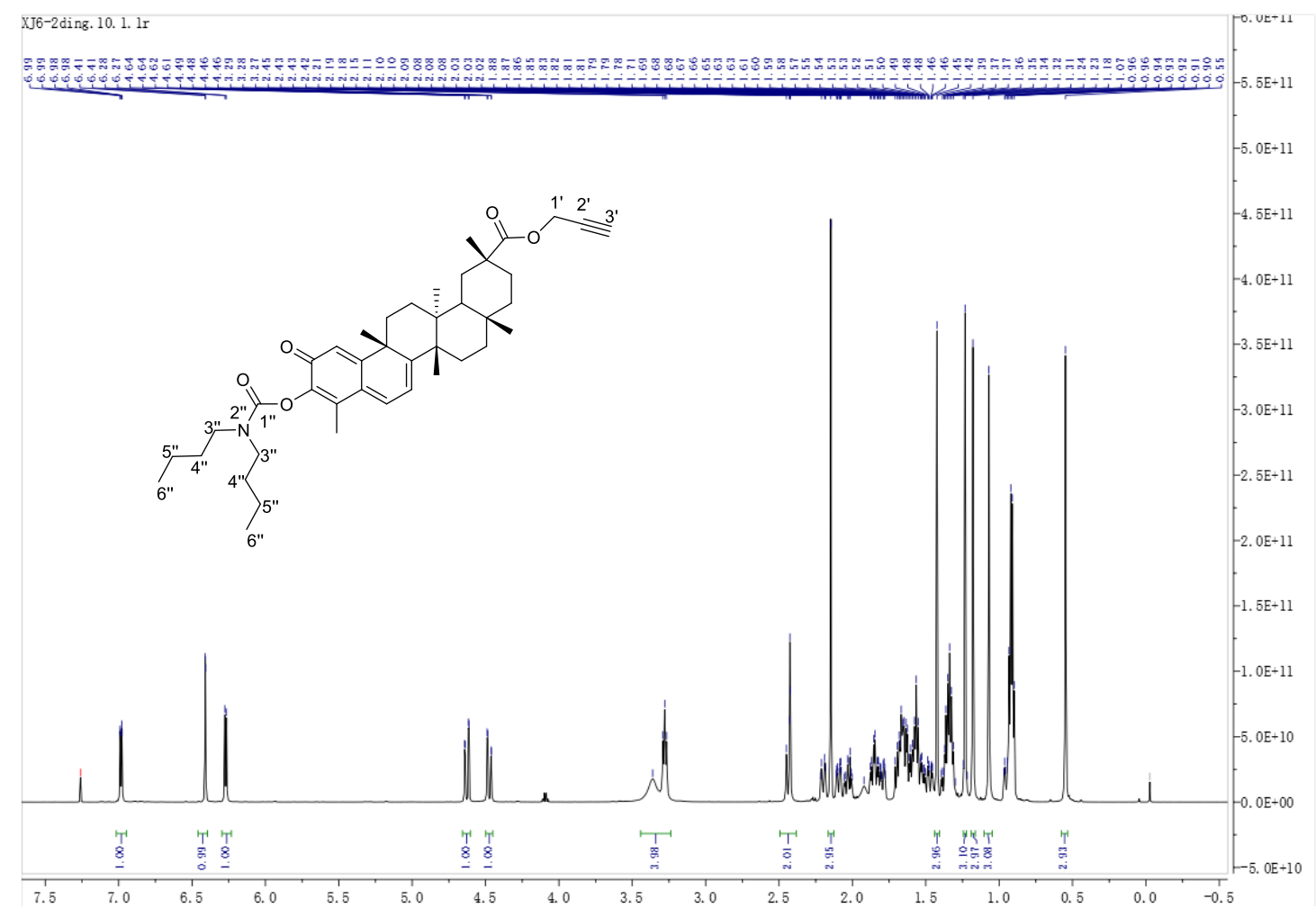

Figure S 26-1 ${ }^{1} \mathrm{H}$ NMR Spectrum $(600 \mathrm{MHz})$ of compound 40 in $\mathrm{CDCl}_{3}$ 


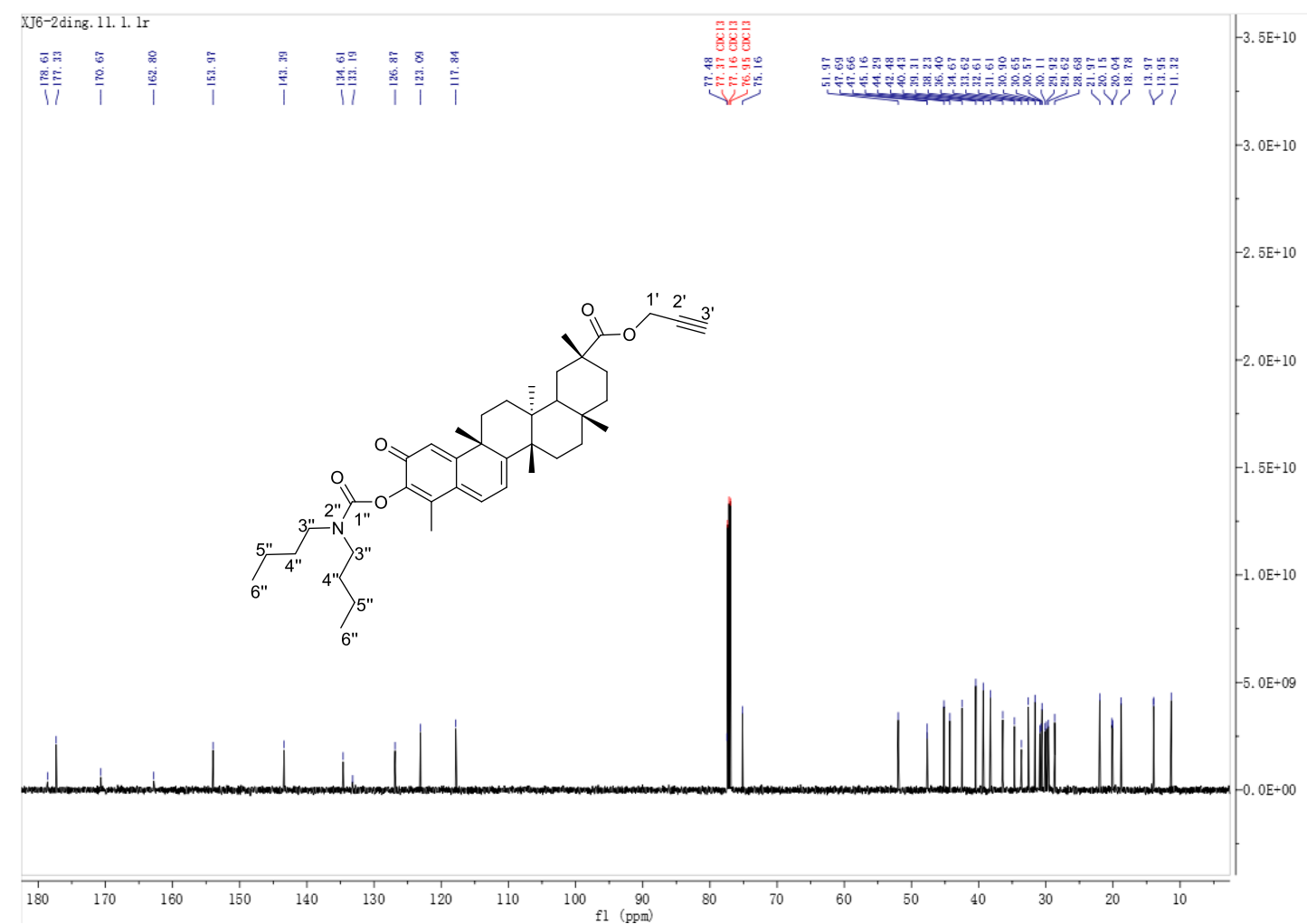

Figure S 26-2 ${ }^{13} \mathrm{C}$ NMR Spectrum $(150 \mathrm{MHz})$ of compound 40 in $\mathrm{CDCl}_{3}$

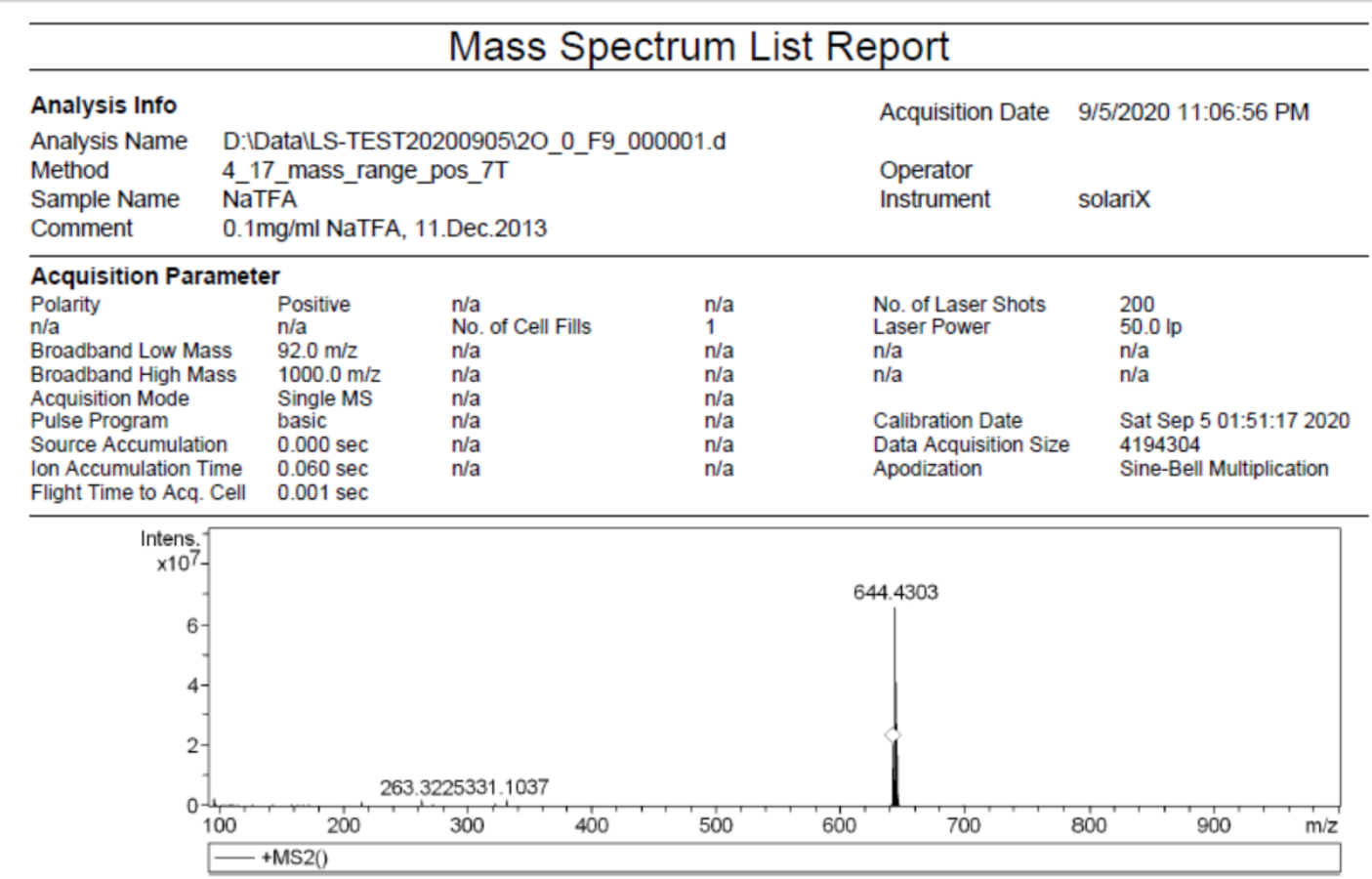

Figure S 26-3 HRESIMS Spectrum of 40 
〈色谱图〉

$\mathrm{mV}$

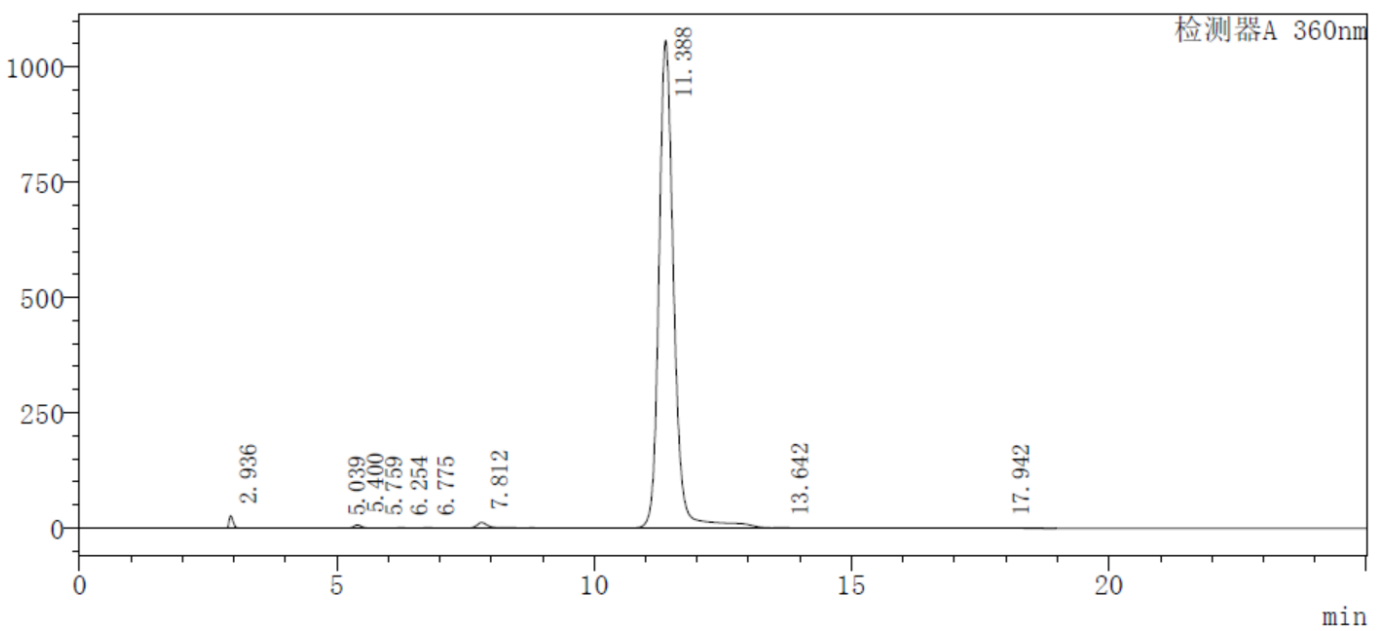

Figure S 26-4 HPLC Spectrum of 4o

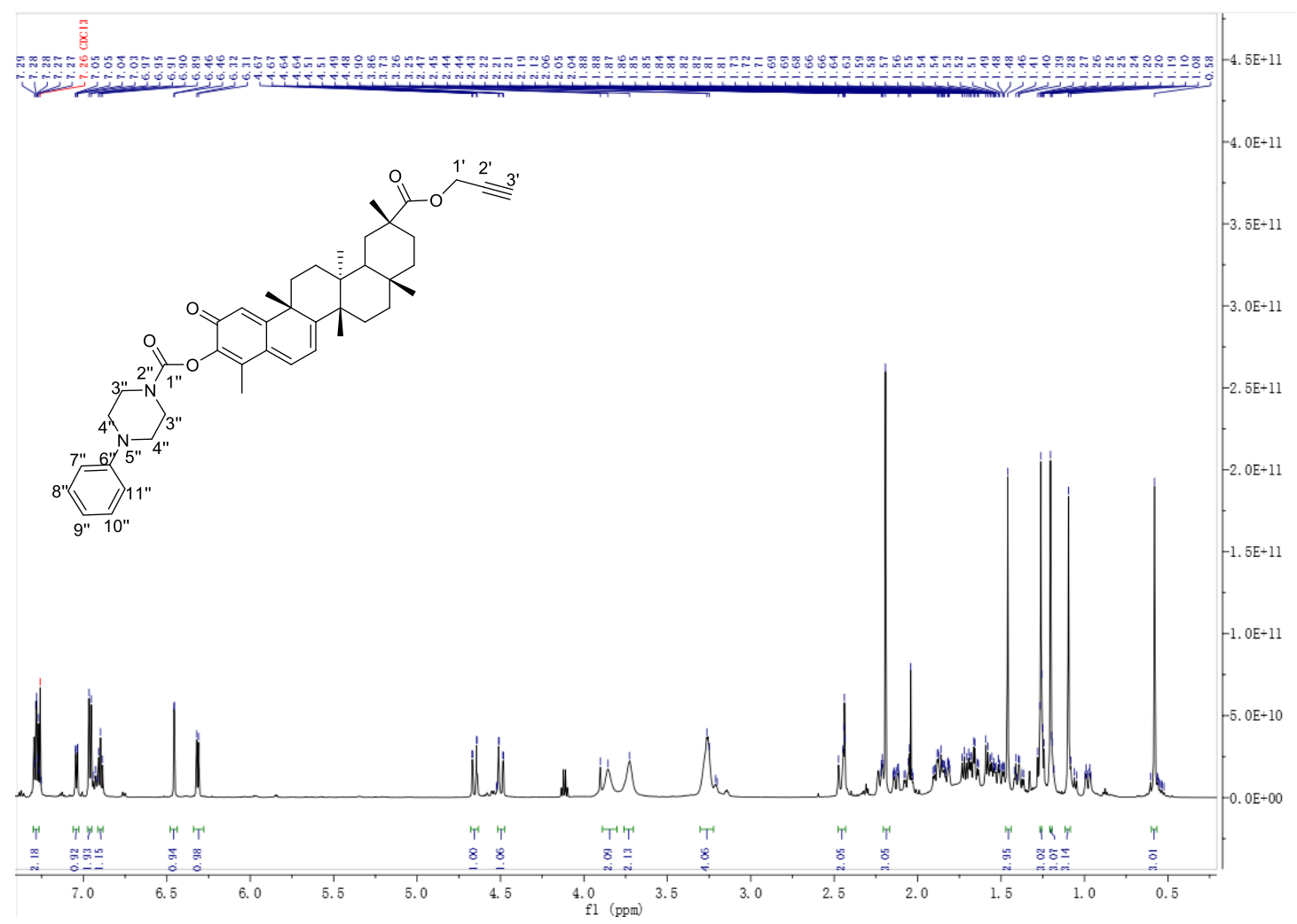

Figure S 27-1 ${ }^{1} \mathrm{H}$ NMR Spectrum $(600 \mathrm{MHz})$ of compound $4 \mathbf{p}$ in $\mathrm{CDCl}_{3}$ 


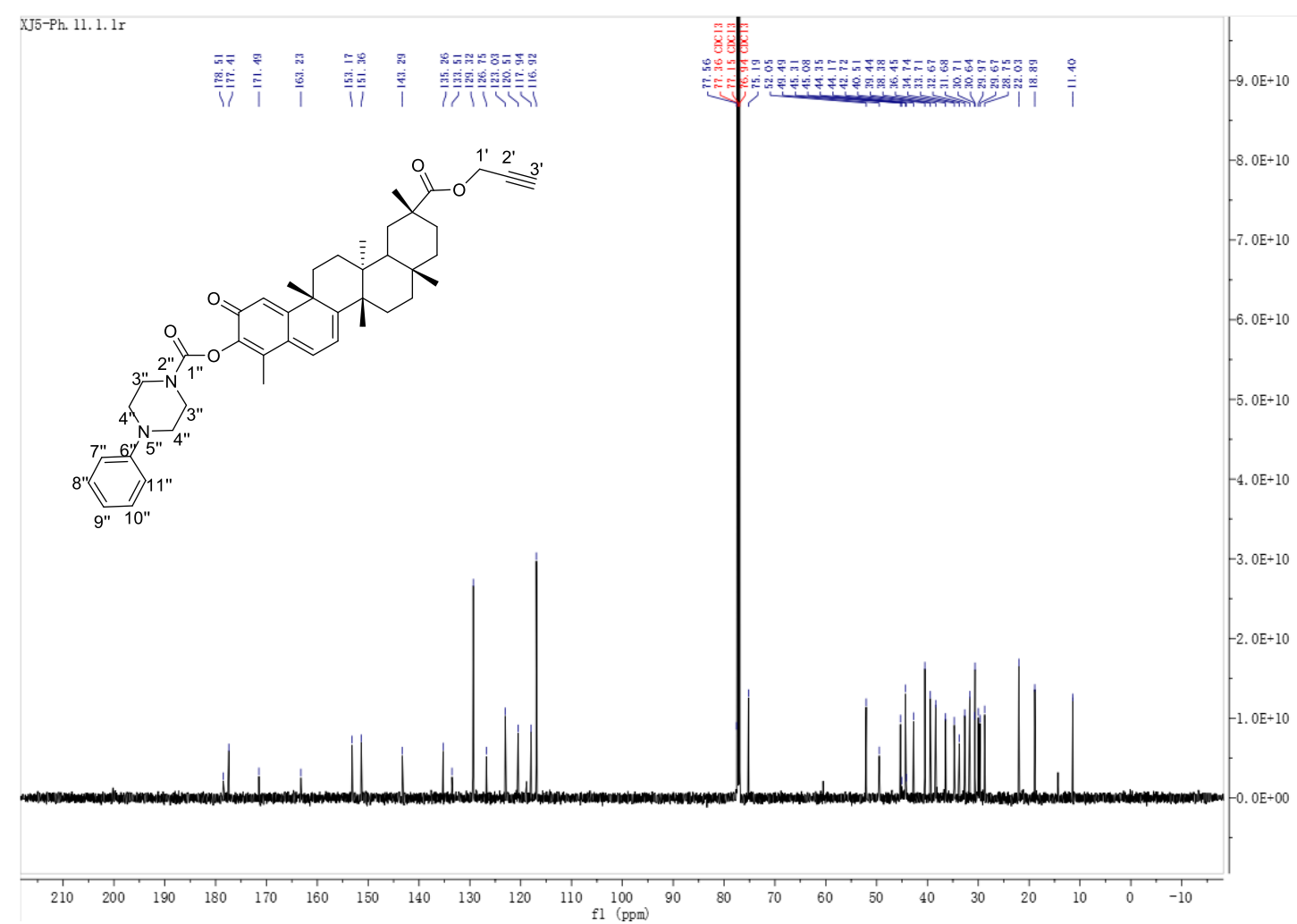

Figure S 27-2 ${ }^{13} \mathrm{C}$ NMR Spectrum $(150 \mathrm{MHz})$ of compound $4 \mathbf{p}$ in $\mathrm{CDCl}_{3}$

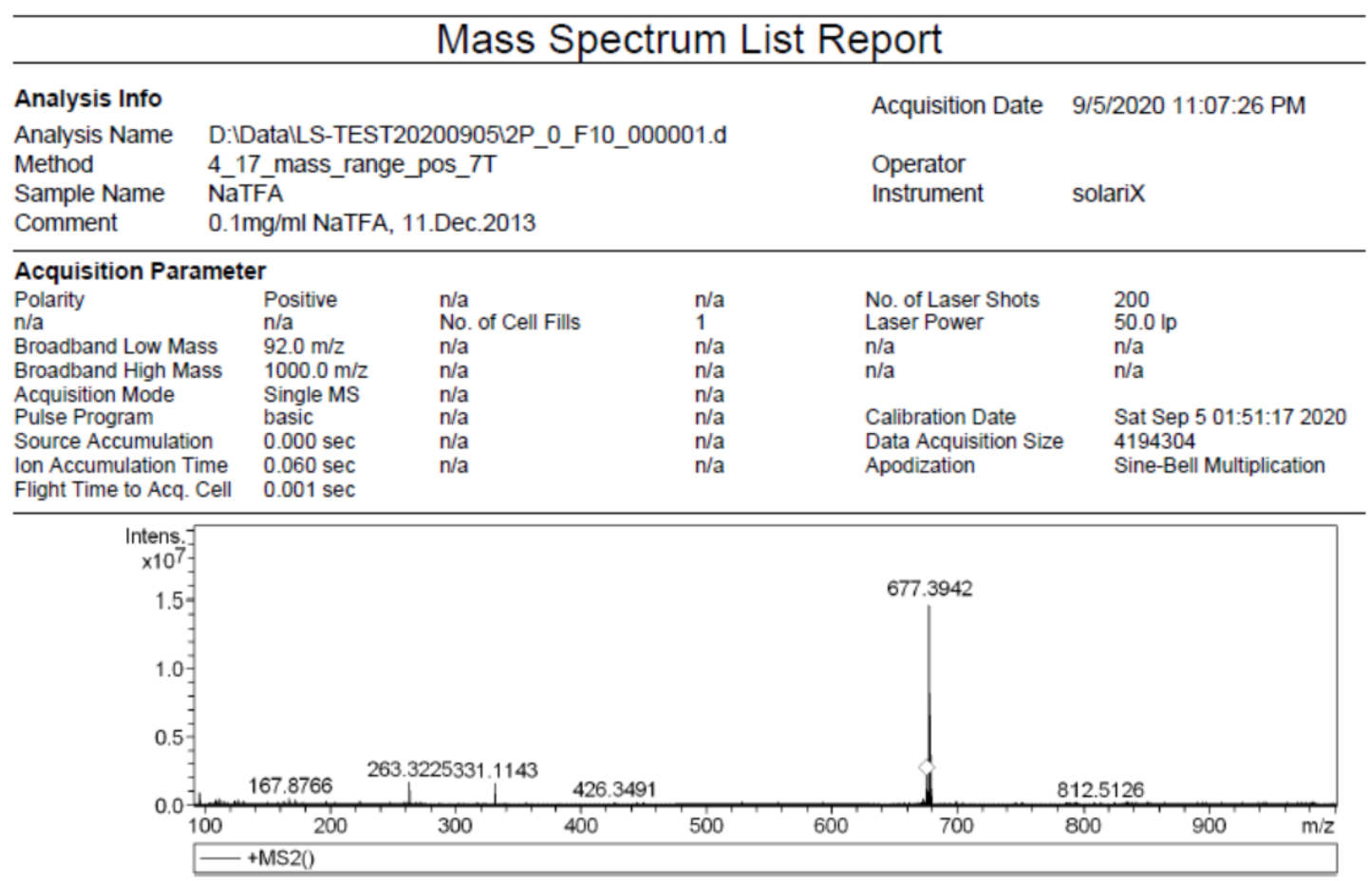

Figure S 27-3 HRESIMS Spectrum of 4p 
〈色谱图〉

$\mathrm{mV}$

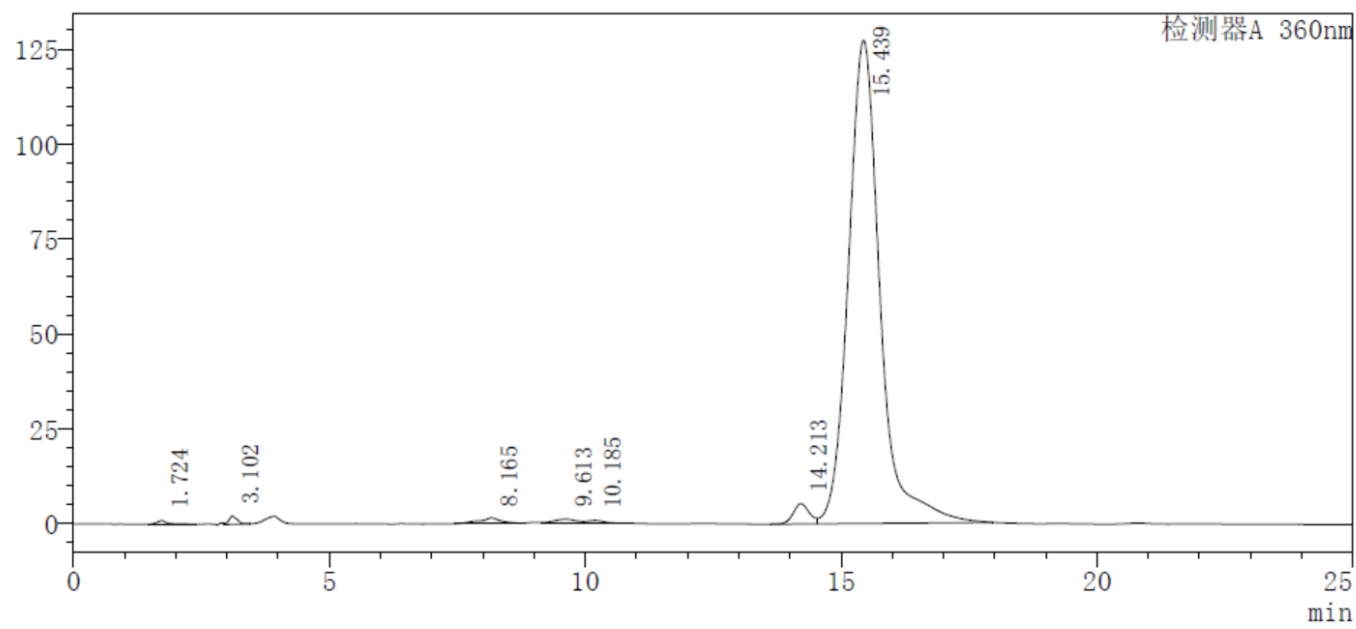

Figure S 27-4 HPLC Spectrum of 4p

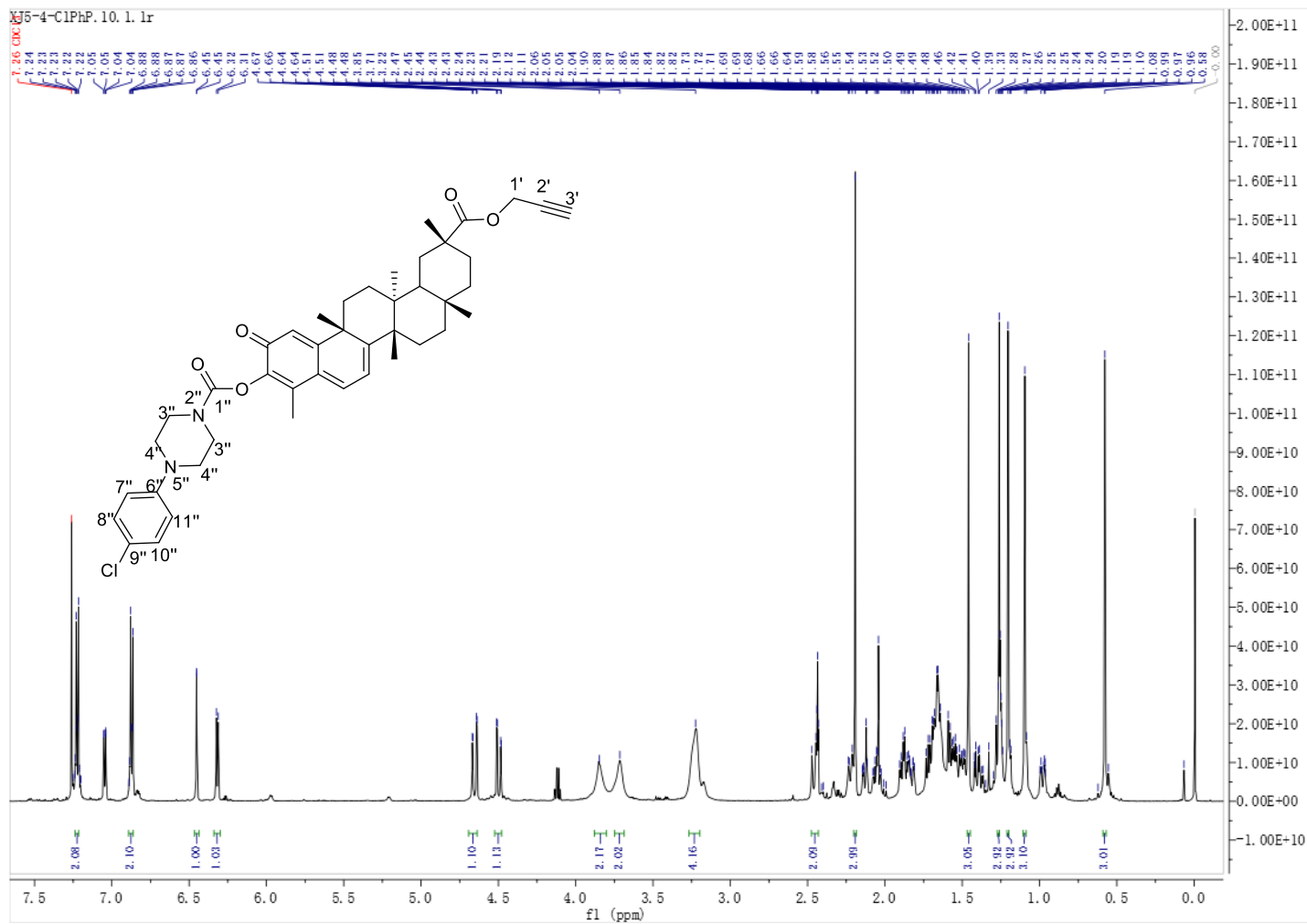

Figure S 28-1 ${ }^{1} \mathrm{H}$ NMR Spectrum (400MHz) of compound $\mathbf{4 q}$ in $\mathrm{CDCl}_{3}$ 


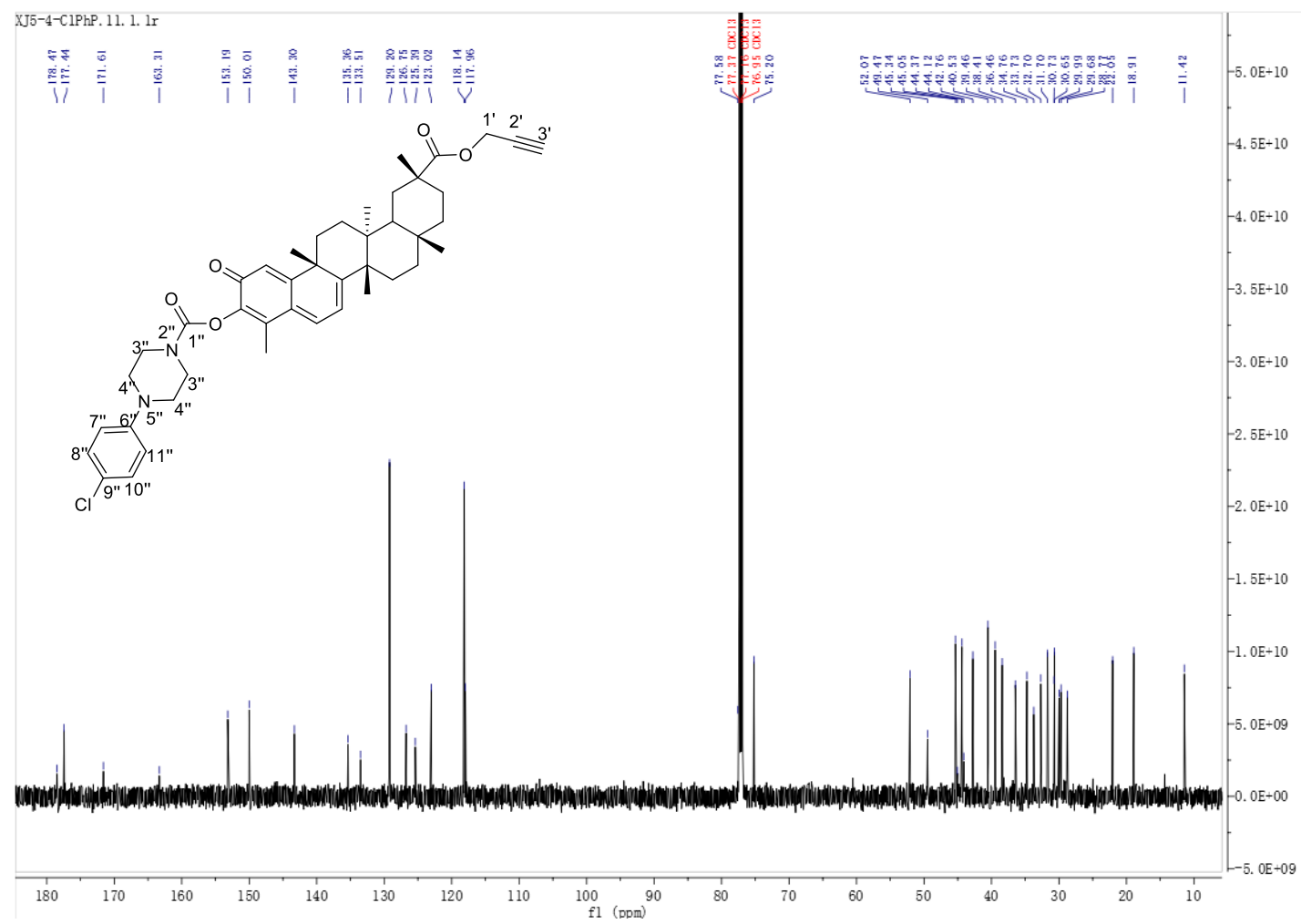

Figure S 28-2 ${ }^{13} \mathrm{C}$ NMR Spectrum $(100 \mathrm{MHz})$ of compound $\mathbf{4 q}$ in $\mathrm{CDCl}_{3}$

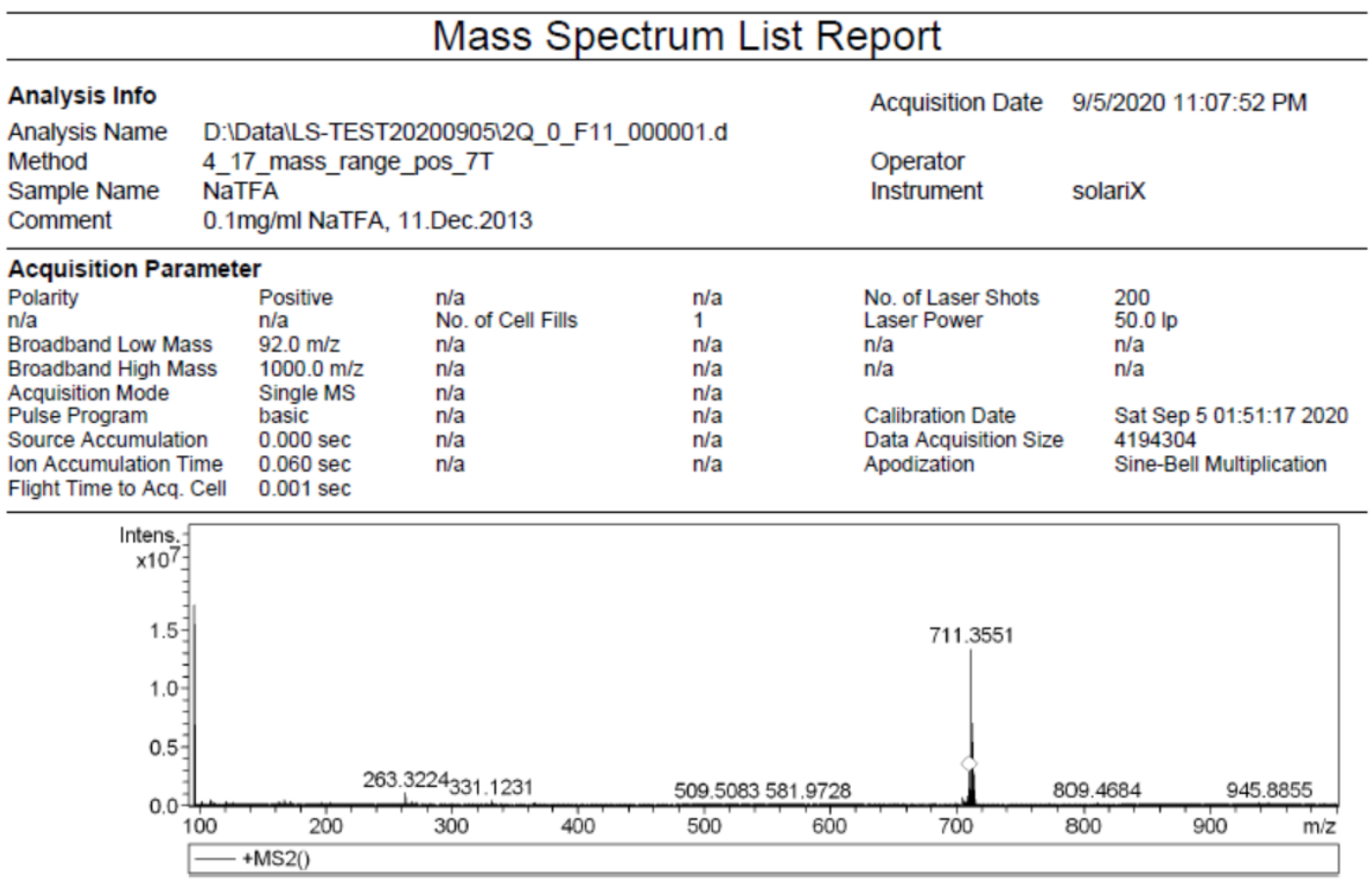

Figure S 28-3 HRESIMS Spectrum of 4q 
〈色谱图〉

$\mathrm{mV}$

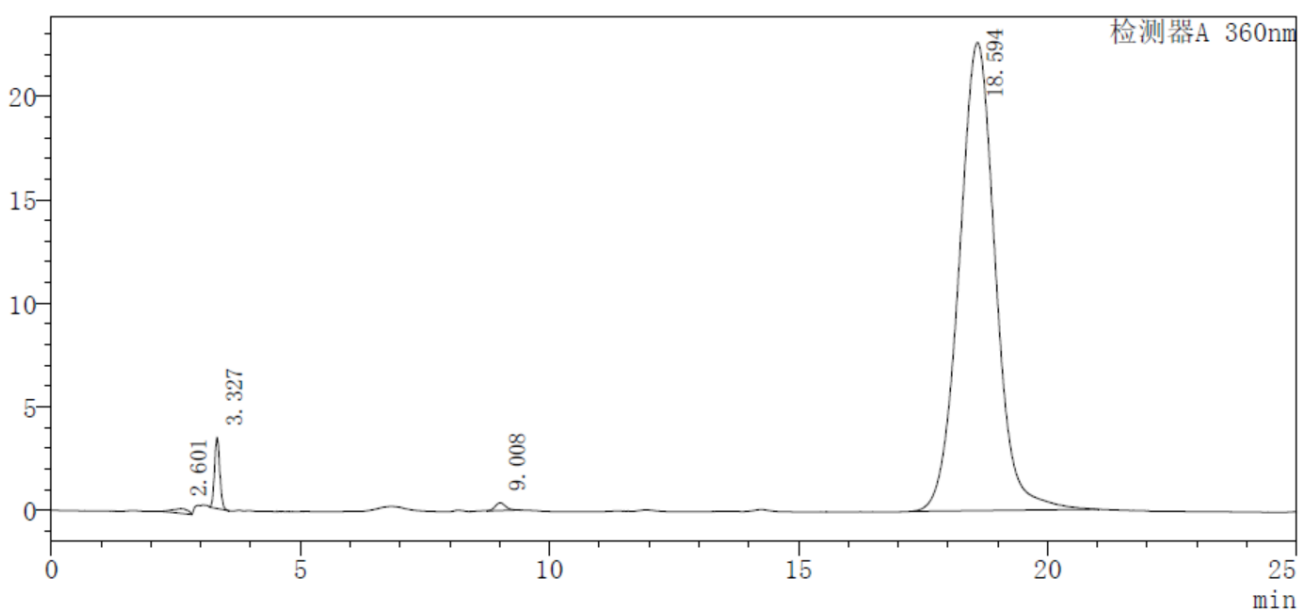

Figure S 28-4 HPLC Spectrum of $\mathbf{4 q}$

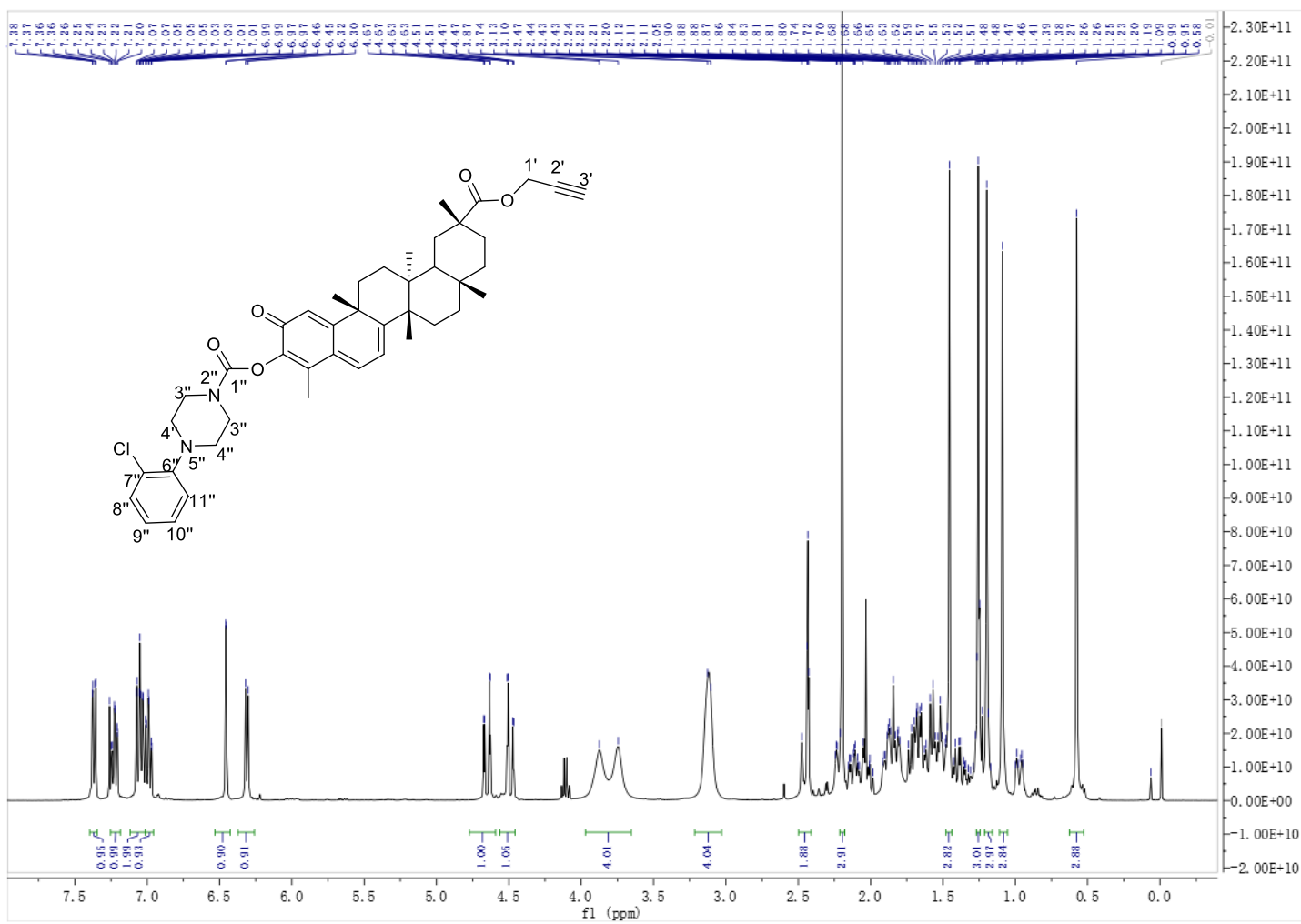

Figure S 29-1 ${ }^{1} \mathrm{H}$ NMR Spectrum (400MHz) of compound $4 r$ in $\mathrm{CDCl}_{3}$ 


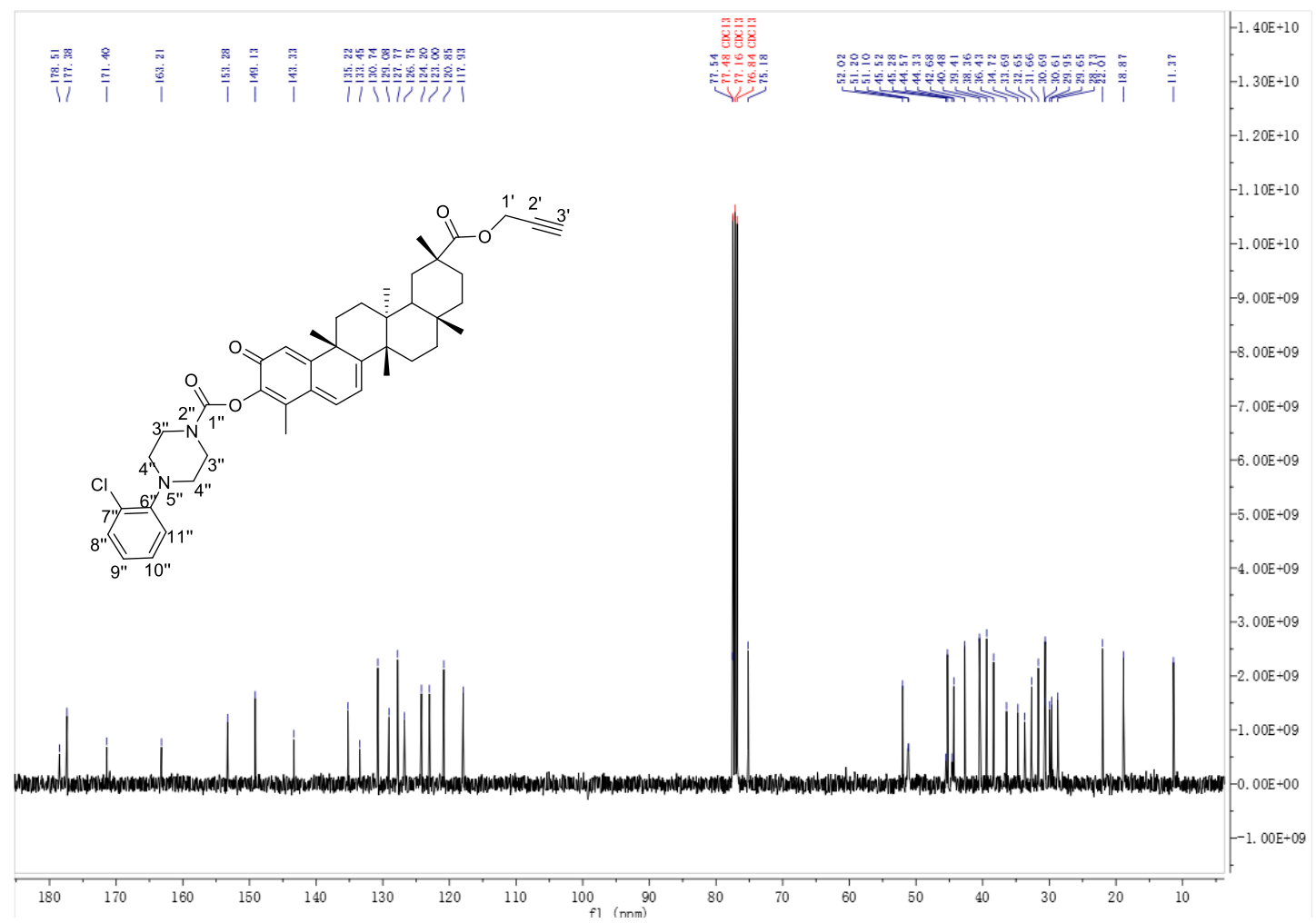

Figure S 29-2 ${ }^{13} \mathrm{C}$ NMR Spectrum $(100 \mathrm{MHz})$ of compound $4 \mathbf{r}$ in $\mathrm{CDCl}_{3}$

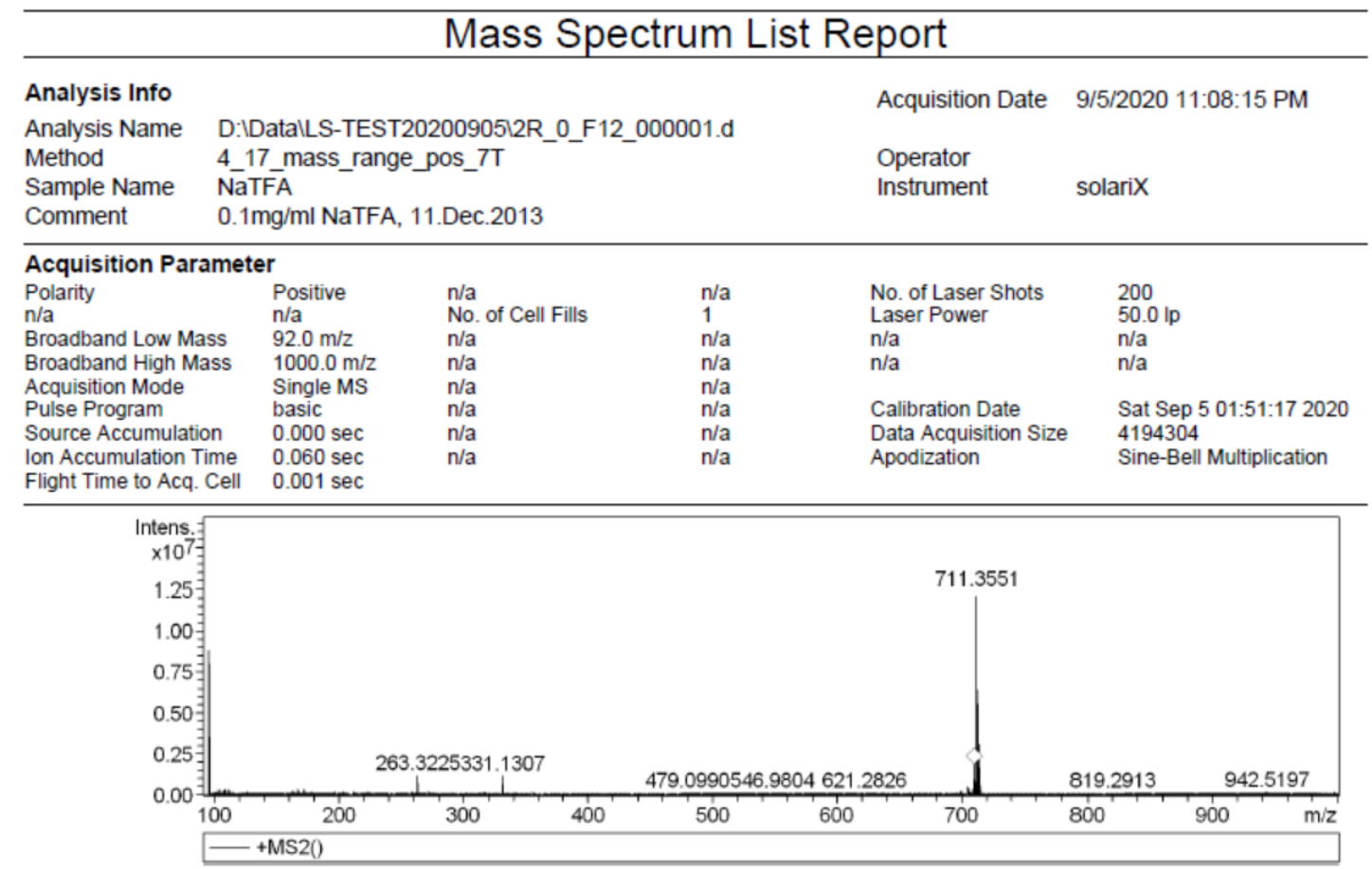

Figure S 29-3 HRESIMS Spectrum of 4r 
〈色谱图〉

$\mathrm{mV}$

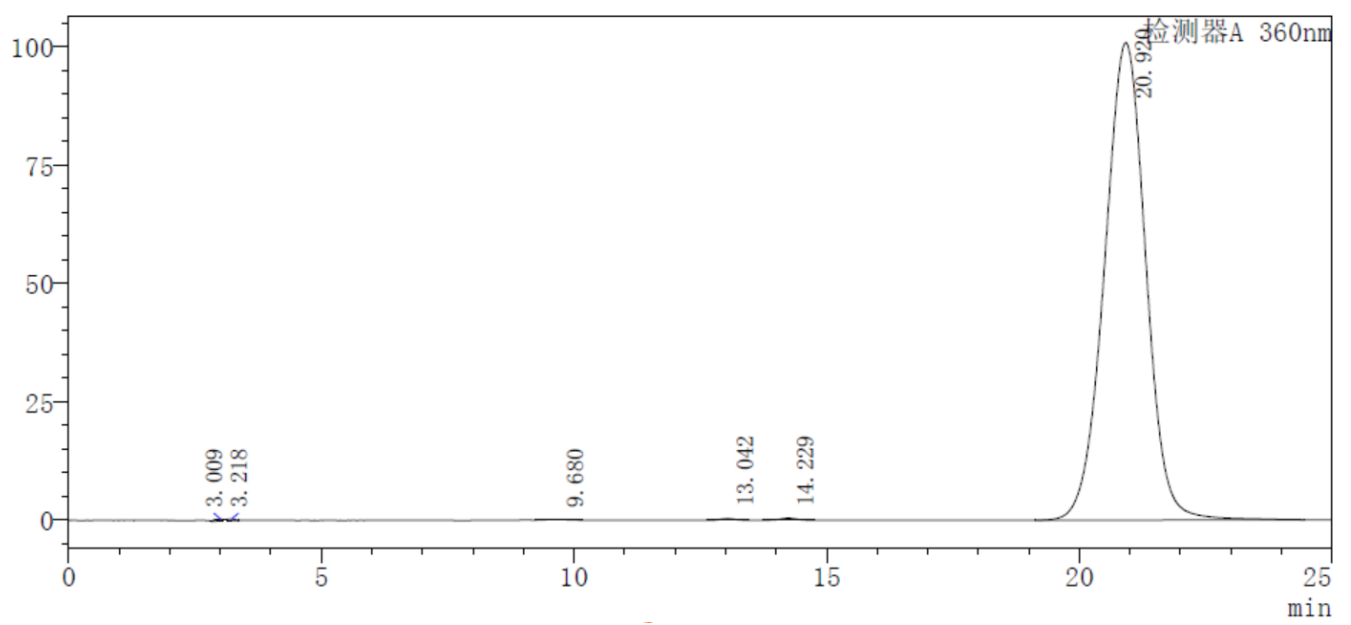

Figure S 29-4 HPLC Spectrum of 4r

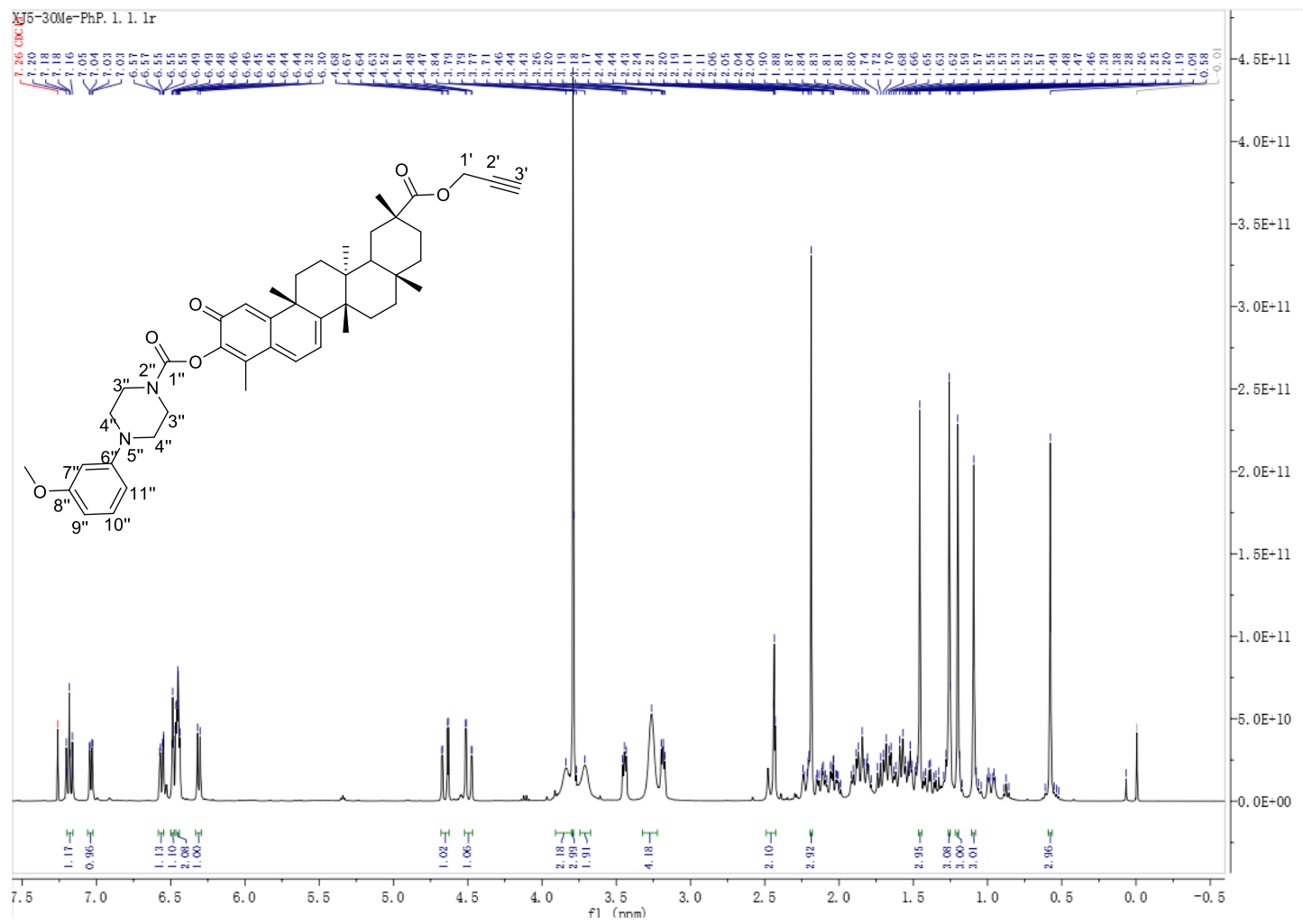

Figure S 30-1 ${ }^{1} \mathrm{H}$ NMR Spectrum $(400 \mathrm{MHz})$ of compound $4 \mathrm{~s}$ in $\mathrm{CDCl}_{3}$ 


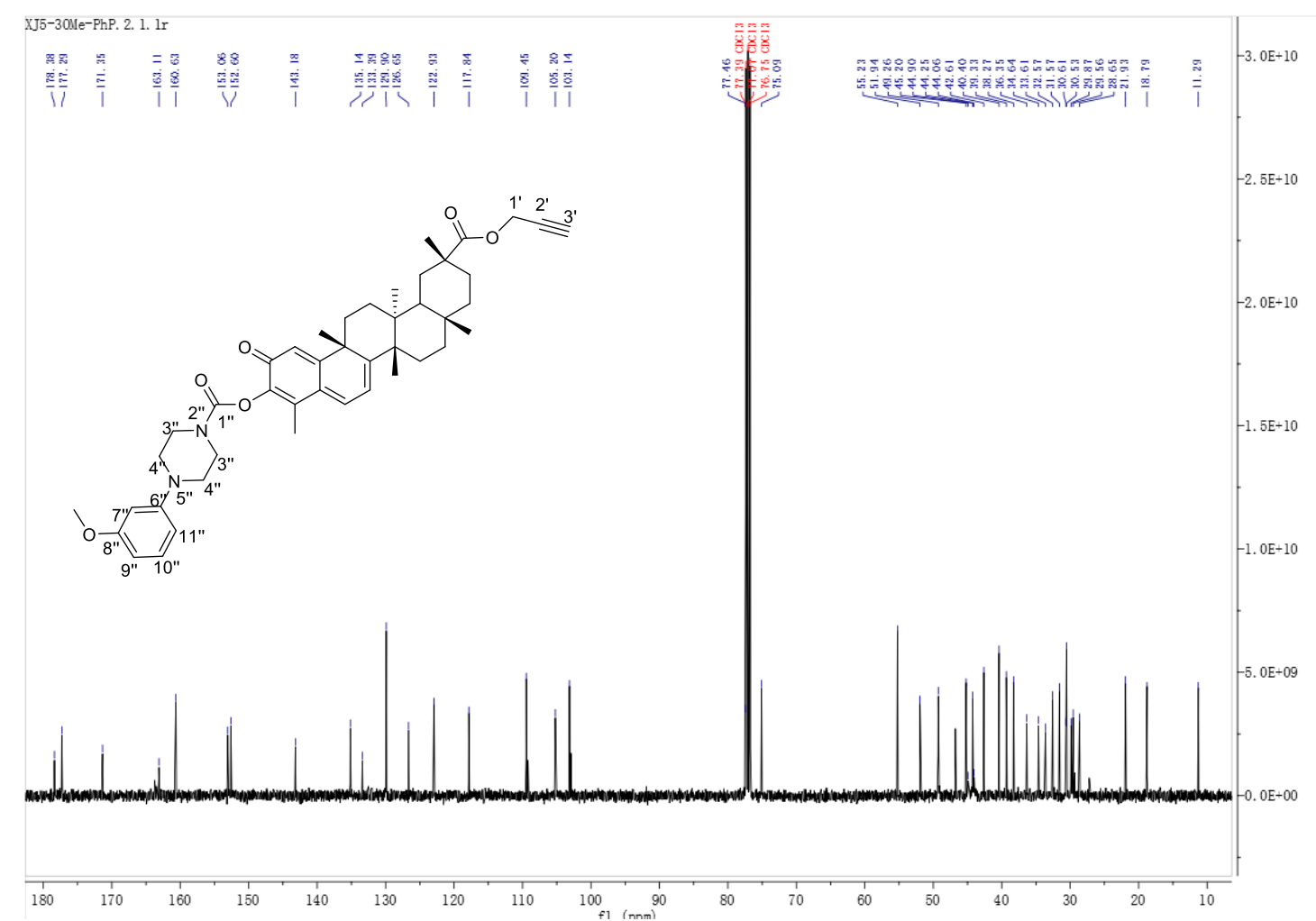

Figure S 30-2 ${ }^{13} \mathrm{C}$ NMR Spectrum $(100 \mathrm{MHz})$ of compound $4 \mathbf{s}$ in $\mathrm{CDCl}_{3}$

\section{Mass Spectrum List Report}

Analysis Info

Analysis Name Method

Sample Name Comment

Acquisition Date $\quad$ 9/5/2020 11:08:35 PM

D:IDatalLS-TEST2020090512S_0_F13_000001.d 4_17_mass_range_pos_7T

Operator

Instrument solariX

$0.1 \mathrm{mg} / \mathrm{ml}$ NaTFA, 11.Dec. 2013

\section{Acquisition Parameter}

Polarity $\mathrm{n} / \mathrm{a}$

Broadband Low Mass

Broadband High Mass

Acquisition Mode

Pulse Program

Source Accumulation

Ion Accumulation Time

Positive

$\mathrm{n} / \mathrm{a}$

$1000.0 \mathrm{~m} / \mathrm{z}$

Single MS

basic

$0.000 \mathrm{sec}$

$0.060 \mathrm{sec}$

$0.001 \mathrm{sec}$

n/a
No. of Cell Fills
n/a
n/a
n/a
n/a
n/a
n/a

$\mathrm{n} / \mathrm{a}$

1

n/a

$n / a$
$n / a$
$n / a$

$\mathrm{n} / \mathrm{a}$

$\mathrm{n} / \mathrm{a}$

No. of Laser Shots
Laser Power
n/a
n/a
Calibration Date
Data Acquisition Size
Apodization

$\mathrm{n} / \mathrm{a}$

Sat Sep 5 01:51:17 2020

4194304

Sine-Bell Multiplication

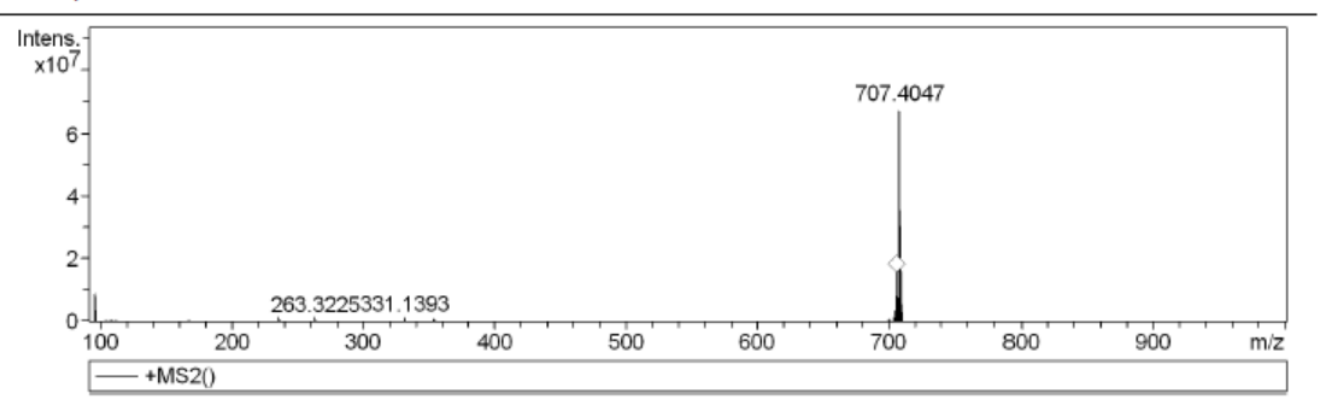

Figure S 30-3 HRESIMS Spectrum of $4 \mathbf{s}$ 
〈色谱图〉

$\mathrm{mV}$

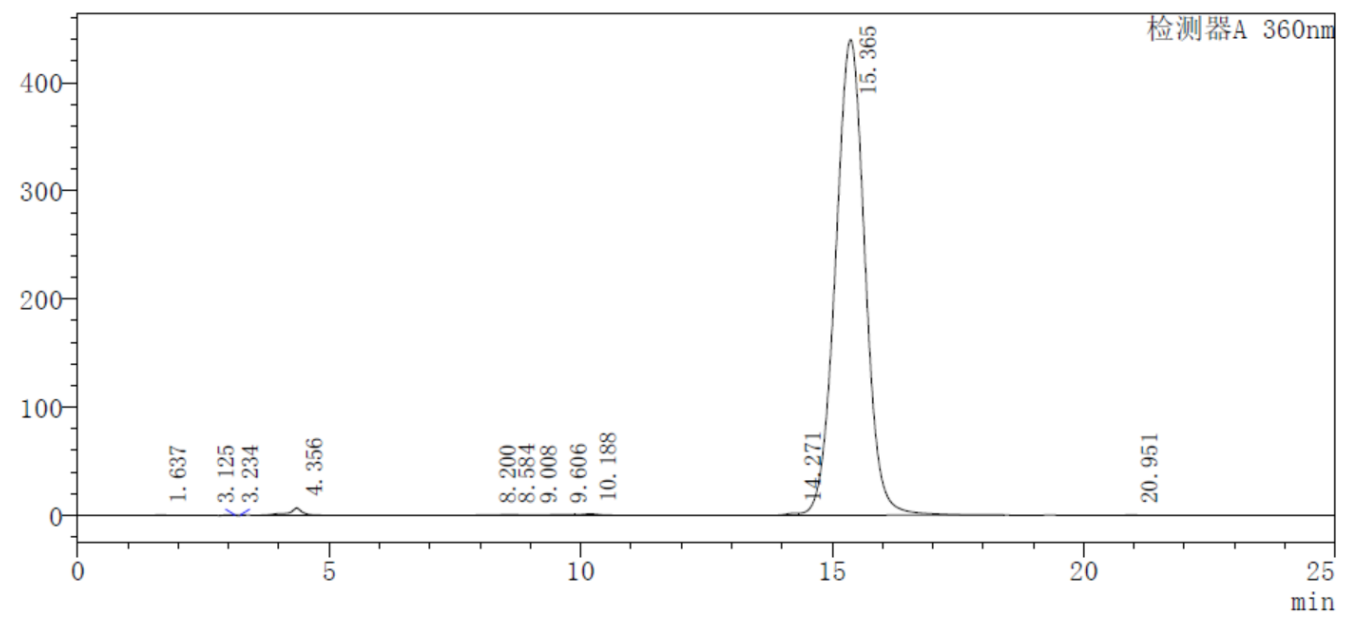

Figure S 30-4 HPLC Spectrum of $4 s$

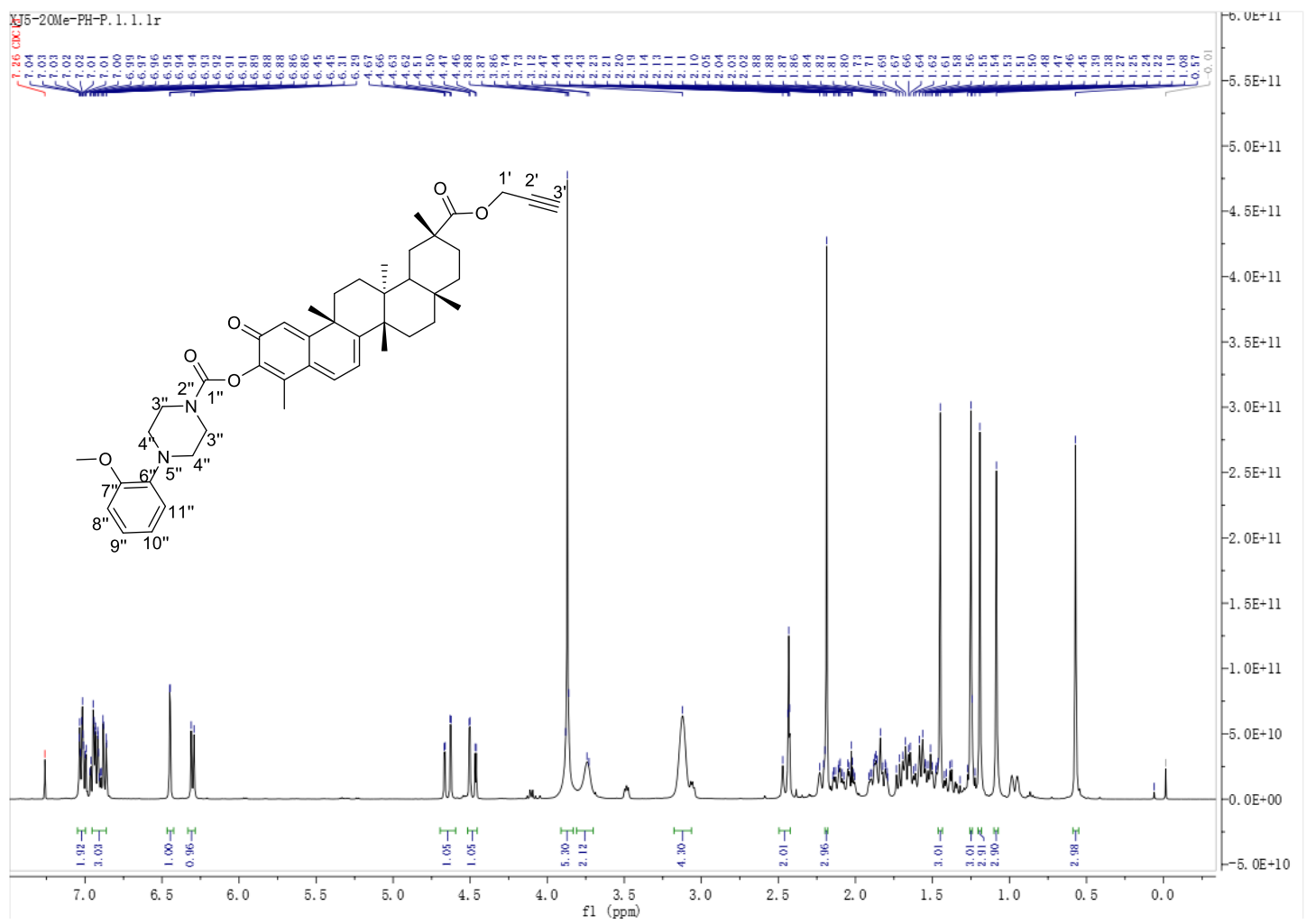

Figure S 31-1 ${ }^{1} \mathrm{H}$ NMR Spectrum (400MHz) of compound $4 \mathrm{t}$ in $\mathrm{CDCl}_{3}$ 


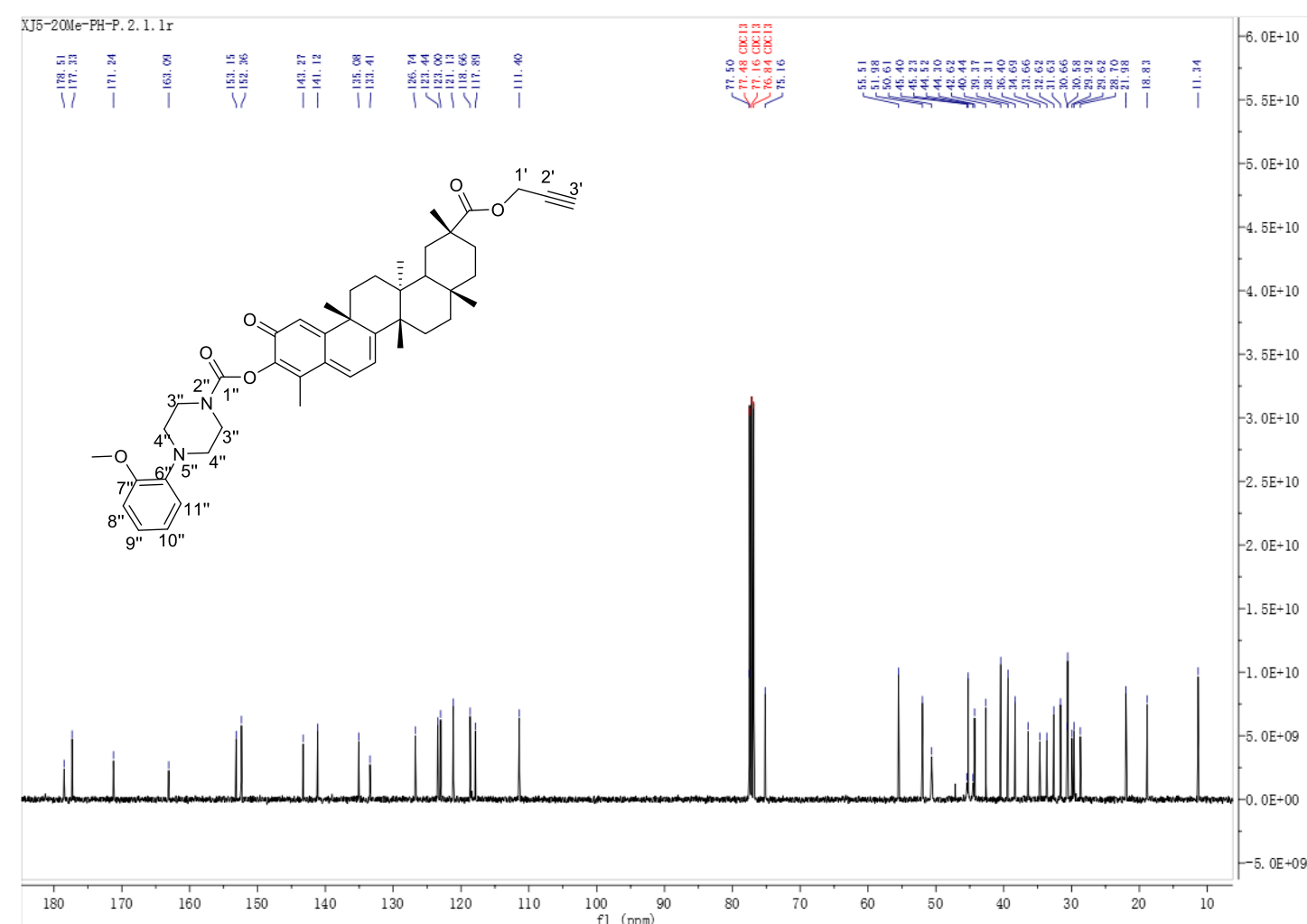

Figure S 31-2 ${ }^{13} \mathrm{C}$ NMR Spectrum $(100 \mathrm{MHz})$ of compound $\mathbf{4 t}$ in $\mathrm{CDCl}_{3}$.

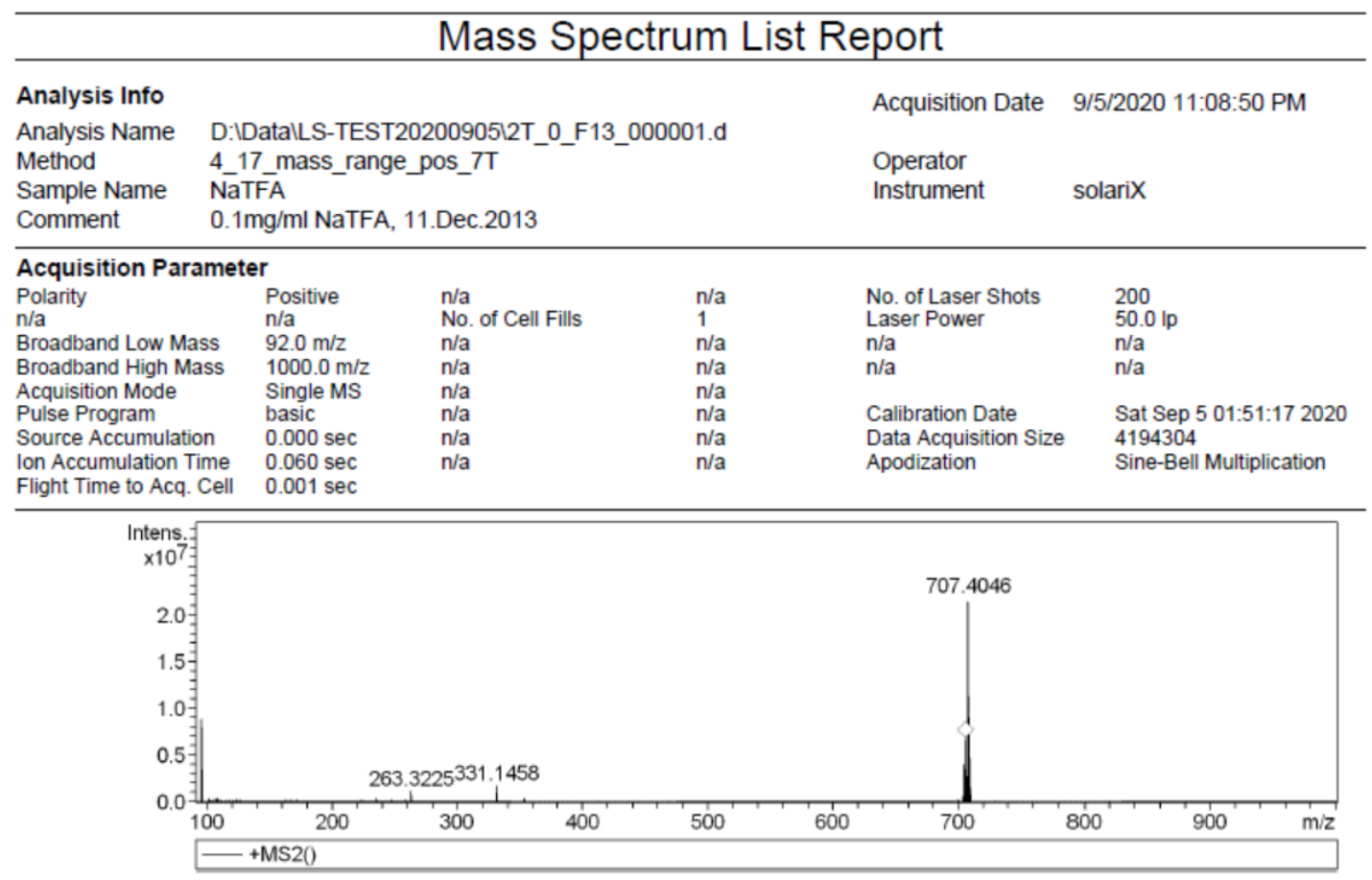

Figure S 31-3 HRESIMS Spectrum of $\mathbf{4 t}$ in $\mathrm{CDCl}_{3}$ 


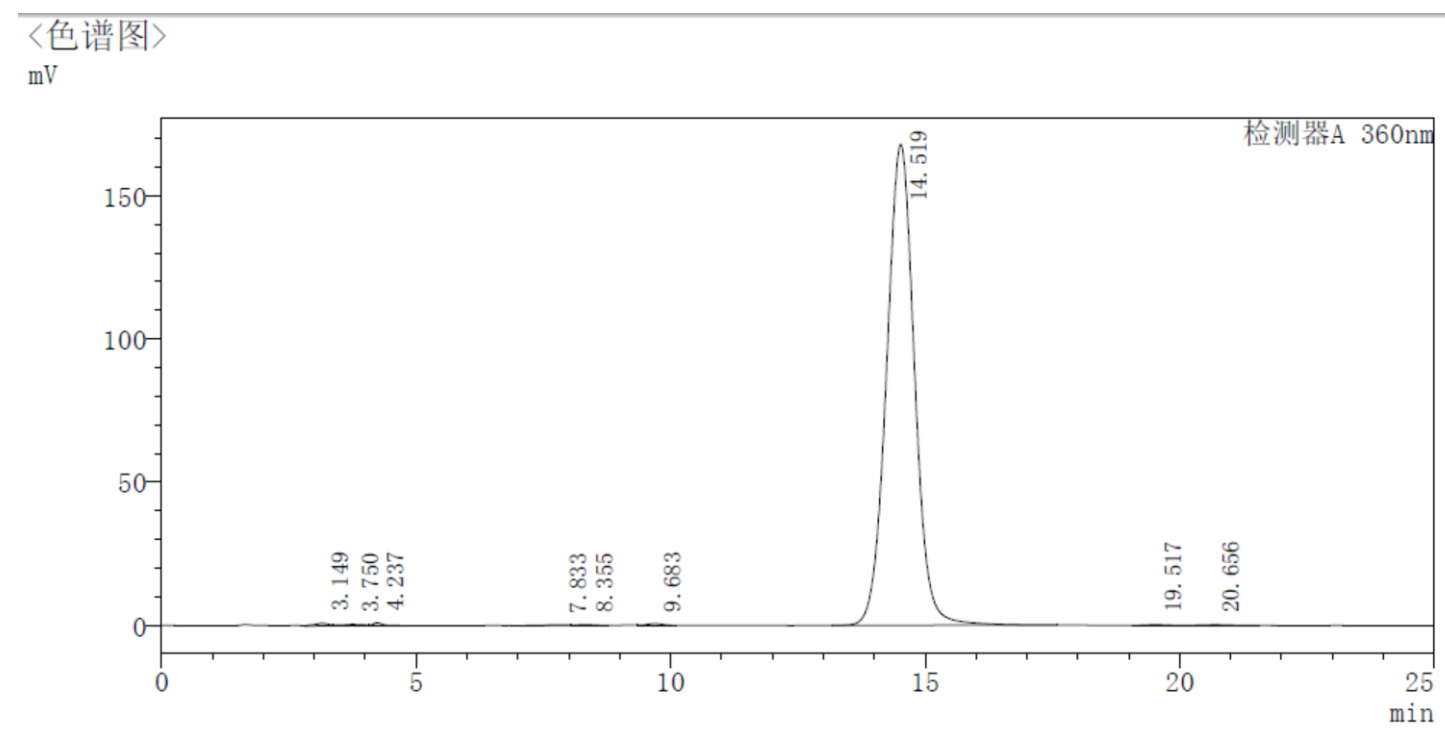

Figure S 31-4 HPLC Spectrum of $\mathbf{4 t}$ in $\mathrm{CDCl}_{3}$ 